\title{
Temporal changes in the epidemiology, treatment and outcome of inflammatory bowel disease in South Limburg
}

Citation for published version (APA):

Jeuring, S. (2019). Temporal changes in the epidemiology, treatment and outcome of inflammatory bowel disease in South Limburg. [Doctoral Thesis, Maastricht University]. https://doi.org/10.26481/dis.20191210sj

Document status and date:

Published: 01/01/2019

DOI:

10.26481/dis.20191210sj

Document Version:

Publisher's PDF, also known as Version of record

Please check the document version of this publication:

- A submitted manuscript is the version of the article upon submission and before peer-review. There can be important differences between the submitted version and the official published version of record.

People interested in the research are advised to contact the author for the final version of the publication, or visit the DOI to the publisher's website.

- The final author version and the galley proof are versions of the publication after peer review.

- The final published version features the final layout of the paper including the volume, issue and page numbers.

Link to publication

\footnotetext{
General rights rights.

- You may freely distribute the URL identifying the publication in the public portal. please follow below link for the End User Agreement:

www.umlib.nl/taverne-license

Take down policy

If you believe that this document breaches copyright please contact us at:

repository@maastrichtuniversity.nl

providing details and we will investigate your claim.
}

Copyright and moral rights for the publications made accessible in the public portal are retained by the authors and/or other copyright owners and it is a condition of accessing publications that users recognise and abide by the legal requirements associated with these

- Users may download and print one copy of any publication from the public portal for the purpose of private study or research.

- You may not further distribute the material or use it for any profit-making activity or commercial gain

If the publication is distributed under the terms of Article $25 \mathrm{fa}$ of the Dutch Copyright Act, indicated by the "Taverne" license above, 


\section{TEMPORAL CHANGES}

\section{IN THE EPIDEMIOLOGY, TREATMENT}

AND OUTCOME OF

INFLAMMATORY BOWEL DISEASE

IN SOUTH LIMBURG 
(c) Copyright S.F.G. Jeuring, Maastricht 2019

All rights reserved. No parts of this thesis may be reproduced or transmitted in any form or by any means, without prior permission in writing by the author, or when appropriate, by the publishers of the publications.

Cover design: ikbenprins

Lay out: Steven Jeuring

Printed by: Print Service Ede

ISBN: 978-90-830276-7-8

The work presented in this thesis was performed within the framework of NUTRIM School of Nutrition and Translational Research in Metabolism [Maastricht University].

Part of this work was supported by funding from the European Union Seventh Framework Programme [FP7/2007-2013], under grant agreement nº 305564

Printing of this thesis was financially supported by: Maastricht University,

Nederlandse Vereniging voor Gastroenterologie, Dr. Falk Pharma Benelux B.V., Ferring B.V. 


\title{
TEMPORAL CHANGES
}

\author{
IN THE EPIDEMIOLOGY, TREATMENT \\ AND OUTCOME OF
}

\section{INFLAMMATORY BOWEL DISEASE \\ IN SOUTH LIMBURG}

\section{PROEFSCHRIFT}

ter verkrijging van de graad van doctor aan de Universiteit Maastricht, op gezag van de Rector Magnificus, prof. dr. Rianne M. Letschert volgens het besluit van het College van Decanen, in het openbaar te verdedigen op

dinsdag 10 december 2019 om 16:00 uur

door 


\section{Promotores}

prof. dr. A.A.M. Masclee

prof. dr. D.M.A.E. Jonkers

\section{Copromotor}

dr. M.J. Pierik

\section{Beoordelingscommissie}

prof. dr. A.E.R.C.H. Boonen, voorzitter

prof. dr. M.A. Spruit

prof. dr. N.D. Bouvy

dr. B. Oldenburg, UMC Utrecht

dr. F.L. Wolters, Viecuri Medisch Centrum Venlo 
voor mijn ouders voor Lian, Evi en Marin 



\section{Table of contents}

Chapter 1 General introduction

PART I Epidemiology of inflammatory bowel disease in South Limburg

Chapter 2 Cohort profile:

The inflammatory bowel disease South Limburg cohort [IBDSL]

Chapter 3 A 20-year temporal change analysis in incidence, presenting phenotype and mortality in the Dutch IBDSL Cohort

Chapter 4 Epidemiology and long-term outcome of inflammatory bowel disease diagnosed at elderly age - an increasing distinct entity?

PART II Impact of advances in IBD management: new era, better future?

Chapter 5 Improvements in the long-term outcome of Crohn's disease over the 101 past two decades and the relation to changes in medical management

Chapter 6 Disease outcome of ulcerative colitis in an era of changing treatment strategies - Results from the Dutch population-based IBDSL cohort

Chapter 7 Corticosteroid sparing in inflammatory bowel disease is more often achieved in the immunomodulator and biological era

Chapter 8 Time trends in the epidemiology and outcome of perianal fistulizing Crohn's disease in a population-based cohort

Chapter 9 Real-world long-term effectiveness and comparative effectiveness of infliximab and adalimumab in a population-based cohort of Crohn's disease patients

Chapter 10 General discussion

\section{Addendum}

Summary

Nederlandse samenvatting

Valorization

List of publications

Dankwoord 



\section{CHAPTER 1}

General introduction 


\section{General introduction}

May 13th, 1932, during the 83rd session of the American Medical Association, a paper was presented by Crohn, Ginzburg, and Oppenheimer, entitled 'Regional enteritis, a pathological and clinical entity'. 'They described for the first time a benign, chronic, granulomatous disease of the small intestine, based on 14 cases, which was different from intestinal tuberculosis. This disease was described as "an ulcerative disease of the intestinal mucosa, accompanied by disproportionate connective tissue reactions of the remaining walls of the involved intestine, a process which frequently leads to stenosis of the lumen of the intestine and is associated with multiple fistulas". The outcome was unfavorable: "the constant occurrence of a mass in the right iliac fossa usually requires surgical intervention [resection]".

\section{An introduction to inflammatory bowel disease}

Inflammatory bowel disease [IBD] is an umbrella term for chronic inflammatory diseases of the gastrointestinal tract, including Crohn's disease [CD], ulcerative colitis [UC] and IBD unclassified [IBD-U]. The latter is a rest group of inflammatory intestinal diseases that cannot be classified as CD, nor UC. ${ }^{2}$ Currently, these bowel diseases are considered separate entities, although some propose they must be seen as different phenotypes of the same inflammatory condition of the bowel.

In CD, inflammatory lesions have a patchy distribution and can be present throughout the entire gastrointestinal tract, although the terminal ileum and colon are most frequently affected. The inflammation is transmural and, consequently, CD patients are prone to develop complications, such as intestinal strictures and fistulas. ${ }^{3,4}$ Fistula tracts can develop between intestinal segments, between the intestine and the bladder or vagina, or can traverse the skin in the form of an enterocutaneous or perianal fistula. ${ }^{5}$ In UC, on the other hand, the inflammatory lesions are limited to the colon and spread proximally in a continuous fashion, starting directly at the anal margin. The inflammatory process involves only the superficial mucosal layer. Therefore, in UC, strictures and fistulas are rare. ${ }^{2}$

Patients with IBD suffer from symptoms like diarrhea, bloody stools, abdominal pain, urgency, weight loss and fatigue. ${ }^{2}$ The disease is diagnosed based on a combination of clinical, endoscopic, radiological and histological findings. ${ }^{6}$ The disease courses of both CD and UC are characterized by periods in which the disease is active [so-called 'flares'], alternated with quiescent periods ['remission']. ${ }^{7,8}$ Nearly all patients have a disease relapse after the initial clinical episode [10-year relapse rate of 90\% in CD and 83\% in UC], many already within the first year [53\% and 52\%, respectively]. ${ }^{78}$ The disease cannot be cured and patients often require maintenance medical therapy or surgery to treat active disease and to reduce the risk of a relapse. 
Next to intestinal symptoms, 25-40\% of IBD patients have extraintestinal manifestations ${ }^{9}$, including rheumatological [e.g. ankylosing spondylitis, arthritis], dermatological [e.g. erythema nodosum, pyoderma gangrenosum], ocular [e.g. uveitis, episcleritis], and hepatopancreatobiliary [e.g. primary sclerosing cholangitis] diseases. These manifestations can be very invalidating, and their treatment is often challenging.

\section{Inflammatory bowel disease is increasingly common}

The incidence of IBD varies widely across the world, with incidences as low as 0-5 per 100,000 in developing countries and Asia and as high as 20-40 per 100,000 in Western regions such as North America and Europe [Table 1.1].10

Table 1.1. Incidence and prevalence of inflammatory bowel disease throughout the world, presented in quintiles [adapted from: Molodecky et al.. $]^{10}$

\begin{tabular}{lcccc}
\hline & \multicolumn{2}{c}{ Crohn's disease } & \multicolumn{2}{c}{ Ulcerative colitis } \\
& $\begin{array}{c}\text { Incidence } \\
\text { per 100,000 }\end{array}$ & $\begin{array}{c}\text { Prevalence } \\
\text { per 100,000 }\end{array}$ & $\begin{array}{c}\text { Incidence } \\
\text { per 100,000 }\end{array}$ & $\begin{array}{c}\text { Prevalance } \\
\text { per 100,000 }\end{array}$ \\
\hline 0-19th percentile & $0.0-0.80$ & $0.60-6.75$ & $0.0-1.85$ & $2.42-21.0$ \\
20th-39th percentile & $0.81-1.94$ & $6.76-25.0$ & $1.86-3.09$ & $21.1-44.3$ \\
40th-59th percentile & $1.95-3.76$ & $25.1-48.0$ & $3.10-4.97$ & $44.4-100.9$ \\
60th-79th percentile & $3.77-6.38$ & $48.1-135.6$ & $4.98-7.71$ & $101.0-198.0$ \\
80th-100th percentile & $6.39-29.3$ & $135.7-318.5$ & $7.72-19.2$ & $198.1-298.5$ \\
\hline
\end{tabular}

A recent study, based on data from multiple large IBD cohorts, estimated that the current IBD prevalence in Europe is $0.3 \% .{ }^{11}$ Extrapolation of these data to the Dutch situation suggests that approximately 50,000 people in the Netherlands suffer from IBD. However, a report from the nineties on the incidence of IBD in the South Limburg region of the Netherlands revealed that the regional incidence rate is rather high and may equal those from high-incident areas, such as Scandinavia and North America. ${ }^{12,13}$ As such, the current disease burden of IBD in the Netherlands may be underestimated. Moreover, recent epidemiological studies found an increase in the incidence of IBD throughout the world. ${ }^{10}$ Recent data for the Dutch situation are currently lacking, while contemporary information is warranted for patient and physician education, even as for health care planning and budget allocation. 


\section{Inflammatory bowel disease has a complex etiology}

Eighty-four years after Crohn et al. stated that the 'etiology of the disease is unknown', the exact underlying mechanisms contributing to the onset of IBD are still not completely clarified. Current evidence indicates that IBD is caused by "a dysregulated immune response towards intestinal microbiota in a genetically susceptible host", pointing to a complex interplay between the immune system, gut microbiome and environmental factors. ${ }^{14}$ Genetic studies have first identified single nucleotide polymorphisms [SNP's] in the CARD15 locus as a risk factor for IBD. ${ }^{15,16}$ This gene encodes for the NOD2 protein, which plays an important role in the recognition of bacterial peptidoglycans and subsequent local regulation of inflammatory cytokine production. ${ }^{17}$ At present, genome-wide-association-studies [GWAS] have identified more than 240 risk loci associated with IBD, often linked to the innate or adaptive immunity or to intestinal barrier function. ${ }^{18}$ Although this information resulted in the identification of relevant cytokines and pathways in the etiology and pathogenesis of $I B D$, the implications for clinical practice are currently limited. For instance, up to now, no genetic polymorphisms are known to predict disease course or treatment response.

Epidemiological studies increasingly support the hypothesis that environmental factors play a pivotal role in the etiology of IBD. First, the rather low concordance rates of IBD between monozygotic [58.3\% in CD and 18.2\% in UC] and dizygotic [0\% vs. 4.5\%, respectively] twins illustrate the important role of environmental factors must play. ${ }^{19}$ Second, the remarkable difference in IBD incidence between Western and non-Western countries points to the so-called 'Western lifestyle' as an important risk factor for IBD. ${ }^{20,21}$ 'Western lifestyle' is an umbrella term, lacking a clear, uniform definition. Yet, it encompasses diet, industrialization, urbanization and sanitation. The important role of lifestyle is supported by the observation that migrants from low-incident areas were found to acquire the IBD risk from their new area of residency. ${ }^{22-24}$ Another supportive observation is the clear increase in IBD incidence in non-Western countries that adopt a Western lifestyle. ${ }^{10}$ Last, the incidences of CD and UC have increased markedly over just a few decades, which is far to short to explain the rising incidence solely by changing genetic factors. In literature, positive correlations between the onset of IBD and environmental factors such as smoking, air pollution, and diet, have been reported. ${ }^{20,25}$

Gaining insight in the evolution of epidemiological temporal trends in the current era is important to further unravel the etiology of IBD. 
Inflammatory bowel disease is a disease of young adults?

Both $C D$ and $U C$ are generally diagnosed in the prime of patients' lives, with incidence peaks between 20 and 40 years of age, see also Figure 1.1.12 In some Western countries, peak incidences are as high as 36.0 per 100,000 inhabitants for CD and 32.5 per 100,000 inhabitants for UC..$^{10}$

\section{Crohn's disease}

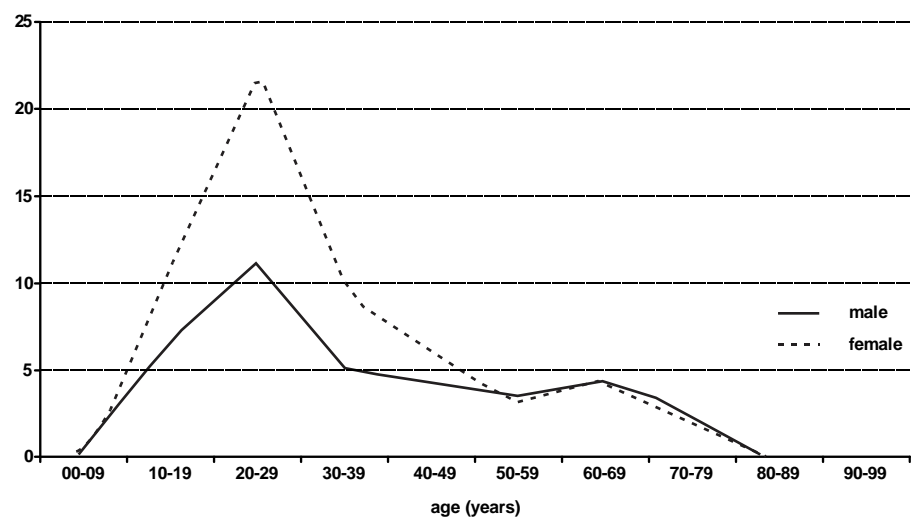

\section{Ulcerative colitis}

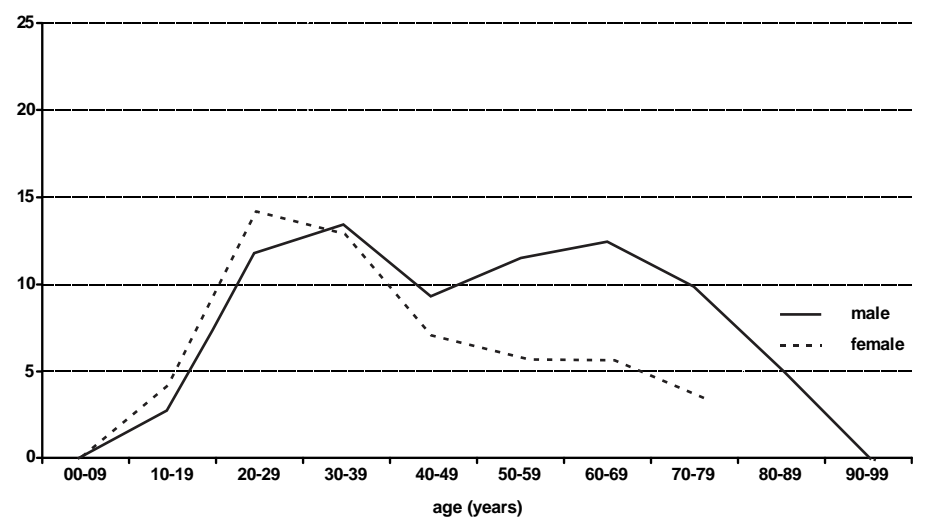

Figure 1.1. Distribution of the standardized incidences [per 100,000] of Crohn's disease and ulcerative colitis among the age spectrum. Adapted from Romberg-Camps et al. ${ }^{12}$ 
IBD with an onset in childhood [between 0 and 18 years of age] is less common; epidemiological studies have estimated that the incidence rate resides between 2 and 8 per 100,000 children. ${ }^{26}$ After the third to fourth decade of life, the risk of developing IBD gradually decreases. Consequently, IBD with an onset at elderly age is not frequently observed. Pooled data from European studies show an incidence of 2.5 per 100,000 for CD and 8.1 per 100,000 for UC at elderly age [= 60 years or older]. ${ }^{27}$ The reason for the age-related incidence attenuation is unknown, but may in part be explained by changes in the functioning of the immune system. This so-called 'immunosenescence' comprises many changes in both cellular and humoral immunity, including a decreased production of hematopoietic stem cells in the bone marrow ${ }^{28}$, a reduction in the number of naïve $T$ cell precursors and an attenuation of [memory] B and T cell activation. ${ }^{28,29}$ This, in turn, may also induce changes in the microbiota composition in the elderly. ${ }^{30-31}$ Despite a gradual decrease in immune function, in some UC cohorts a 'bimodal incidence distribution' has been observed, with a second incidence peak around the age of 60.2,12 Risk factors for this second incidence peak are not well established, but the effect may in part be related to smoking cessation, which is frequently observed in middle-aged men, mainly for cardiovascular reasons. The relation between smoking cessation and the onset of $U C$ is well established. ${ }^{32-34}$

The elderly population will be of increasing importance in the near future. If the rise in IBD incidence also holds for this population, more elderly patients will be diagnosed with IBD. Second, due to population ageing ${ }^{35}$, the proportion of elderly in society increases, which will also contribute to a higher number of elderly patients in our outpatient clinics. Currently, insight in the epidemiology and disease course of elderly-onset IBD is limited and, consequently, it is unknown whether elderly-onset IBD must be considered a distinct entity that must be treated accordingly.

\section{The course of inflammatory bowel disease is heterogeneous}

The course on inflammatory bowel disease is characterized by periods of active mucosal inflammation ['flares'], alternated with periods of remission. The frequency and duration of disease flares vary widely among patients. ${ }^{7,8}$ Recurrent flares and chronic [sub]clinical mucosal inflammation are thought to result in irreversible bowel damage. Such bowel damage includes complications like intestinal strictures and fistulas [in CD], which frequently require hospitalization and surgery. 3.4

In research, it is difficult to study the actual course of the disease, as reliable disease activity parameters are not structurally assessed and recorded in clinical practice, and disease activity is hard to define retrospectively. Consequently, most observational studies use proxy measures for disease severity to study the disease course, such as medication use [in particular corticosteroid use], hospitalization and surgery. ${ }^{7,8,36-51}$ Additionally, in CD, the 
development of strictures and fistulas has become an important outcome measure as it may represent an insufficiently controlled disease. In a landmark paper, Cosnes et al. illustrated that eventually nearly all patients develop these complications, illustrated by cumulative percentages as high as $80-90 \%$ after 20 years of follow-up. ${ }^{52}$

Population-based studies are the optimal cohorts to study disease course, because they comprise the full spectrum of IBD phenotypes, ranging from mild to severe disease. Therefore, external validity is high in such studies. In IBD, most of our current knowledge on the long-term outcomes of $C D$ and $U C$ still mainly derives from population-based cohorts from the eighties and nineties of the past century. Data from the cohorts of IBSEN [Norway $]^{7,8}$, ECIBD [Europe] $]^{27,43,44,49}$, Hungary ${ }^{41,51}$, Cardiff $^{39}$, Denmark ${ }^{38,40,45}$, Manitoba $^{50}$ and others, showed that more than half of the CD patients eventually develop intestinal strictures or fistulas, and that approximately half of the $\mathrm{CD}$ and one-tenth of the $U C$ populations require surgery. These numbers illustrate that a substantial proportion of the IBD population has an unfavorable disease course. Furthermore, IBD patients may suffer from side effects of medical therapy, for instance due to repeated courses of corticosteroids or longstanding immunosuppressive medication. These findings illustrate the importance of optimizing disease management in order to reduce disease burden for IBD patients.

\section{Treatment goals and options in inflammatory bowel disease}

For decades, IBD management was primarily focused on reducing disease-related symptoms. The classic treatment goals are inducing and maintaining clinical disease remission, striving for corticosteroid-sparing management and avoiding surgery. ${ }^{53-55}$ In order to improve the long-term outcome, novel drugs and treatment strategies [e.g. accelerated step-up and topdown] were introduced, and novel treatment goals [e.g. striving for endoscopic or even histological remission] have been proposed.

In the medical treatment of IBD, several medication classes are available.

First, corticosteroids have been a cornerstone for inducing disease remission ever since their availability in the fifties of the past century. They bind to the glucocorticoid receptor, which is present in nearly all human cells, and stimulates the transcription of anti-inflammatory genes [e.g. lipocortin-1, secretory leukocyte protease inhibitor] and suppresses the effects of pro-inflammatory transcription factors [ e.g. nuclear factor $\mathrm{k} \beta[\mathrm{NF}-\mathrm{k} \beta]$, activator protein- 1 ]. ${ }^{56}$ The majority of IBD patients [80\%] show clinical response to corticosteroid therapy. ${ }^{36,57}$ Albeit corticosteroids are highly effective in inducing disease remission, they are not effective as maintenance therapy. ${ }^{58}$ Moreover, serious side effects develop when these drugs are used in high dosages or for long periods of time. Some of these effects are temporary [e.g. acne, Cushingoid symptoms and hyperglycemia], whereas others gradually develop over time and 
are irreversible [e.g. osteoporosis and glaucoma]. ${ }^{59,60}$ Therefore, recurrent episodes of corticosteroid therapy should be limited to the minimum and so-called 'corticosteroid sparing' has become an important goal in IBD management.

Another drug class that has been used for decades to treat IBD is the group of the aminosalicylates [e.g. mesalazine, sulfasalazine, salazopyrine]. They probably exert their effect by a combination of local inhibition of proinflammatory processes [e.g. PPAR- $\delta$, and NF-к $\beta]^{61-}$ 63 and cytokines [e.g. TNF $\alpha$ ], scavenging of free radicals ${ }^{64}$, as well as by other antiinflammatory effects, such as the inhibition of neutrophil and macrophage activity, and T-cell proliferation and activation. ${ }^{65,66}$ While aminosalicylate therapy is still main therapy for UC, its efficacy in CD has been questioned. ${ }^{67}$ Currently, its use in CD is limited to mild colonic disease.

Third, purine analogues are used in the medical management of IBD [e.g. azathioprine, mercaptopurine and tioguanine]. In literature, the term 'immunomodulator' is often used interchangeably, although ciclosporin-A and methotrexate must also be classified as immunomodulator. The first trials with thiopurines in IBD were already conducted in the seventies of the previous century ${ }^{68}$, but these agents mainly gained interest as of the nineties. Thiopurine therapy was first considered second-line therapy after failure of aminosalicylates in both CD and UC. However, recent guidelines advocate thiopurine therapy as first-line maintenance therapy in CD. ${ }^{54}$ Thiopurines interfere with the purine synthesis in lymphocytes ${ }^{69}$ and modulate Ras-related C3 botulinum toxin substrate 1 [Rac1] activation, resulting in decreased numbers of circulating T- and B-lymphocytes and in induction of apoptosis upon activation. ${ }^{70,71}$ Up to the mid-nineties, thiopurine therapy was the last resort of medical therapy [apart from ciclosporin-A in UC as rescue therapy for acute severe colitis]. Failure of thiopurine therapy resulted in the need for surgical resection of the inflamed part of the bowel.

In the late nineties, biological therapy became available and changed the medical management of IBD markedly. The first biologicals were directed towards the proinflammatory cytokine TNF $\alpha$ [anti-TNF $\alpha$ agents]. Interestingly, the clinical effects of anti-TNF $\alpha$ therapy may not be primarily caused by neutralization of the soluble TNF $\alpha$ cytokine, but by inducing apoptosis of T-lymphocytes in the peripheral blood and the lamina propria ${ }^{72}$, and by inducing M2-type macrophages. ${ }^{73}$ Currently, infliximab [a chimeric IgG 1 antibody] ${ }^{74-77}$, adalimumab ${ }^{78-80}$ and golimumab ${ }^{81-82}$ [both fully human IgG 1 antibodies] are registered for IBD treatment in the Netherlands. More recently, generic biologicals [so-called 'biosimilars'] have entered the market. ${ }^{83,84}$ In 2014, vedolizumab was the first agent registered in the class of anti-integrins. ${ }^{85,86}$ It is a humanized monoclonal antibody that binds to the $\alpha 4 \beta 7$ integrin on lymphocytes, thereby inhibiting lymphocyte binding to the mucosal addressin cell adhesion molecule 1 [MAdCAM-1] and subsequent migration through the mucosal layer of the intestine. ${ }^{87}$ As a result, it is thought to provide gut selective anti-inflammatory effects. In 2017, ustekinumab was registered, an interleukin 12/23 inhibitor, inhibiting both Th1 and Th17 
responses. ${ }^{88}$ In this thesis, the clinical effects of the latter two medication classes are not considered, as the follow-up of the present studies ended in 2014, before their availability in the Netherlands.

The medication classes are classically used in a 'step-up' fashion: a sequential introduction of medication after failure of a previous drug class. In general, local and less aggressive therapies are preferred, if allowed by the location and severity of the disease. Under this strategy, topical corticosteroids or budesonide, corticosteroids, immunomodulators, anti-TNF $\alpha$ therapy and surgery form the 'treatment pyramid' [Figure 1.2]. It must be noted that this is a simplification of IBD treatment, because individual patient and disease characteristics, even as therapy availability, may warrant a different approach.

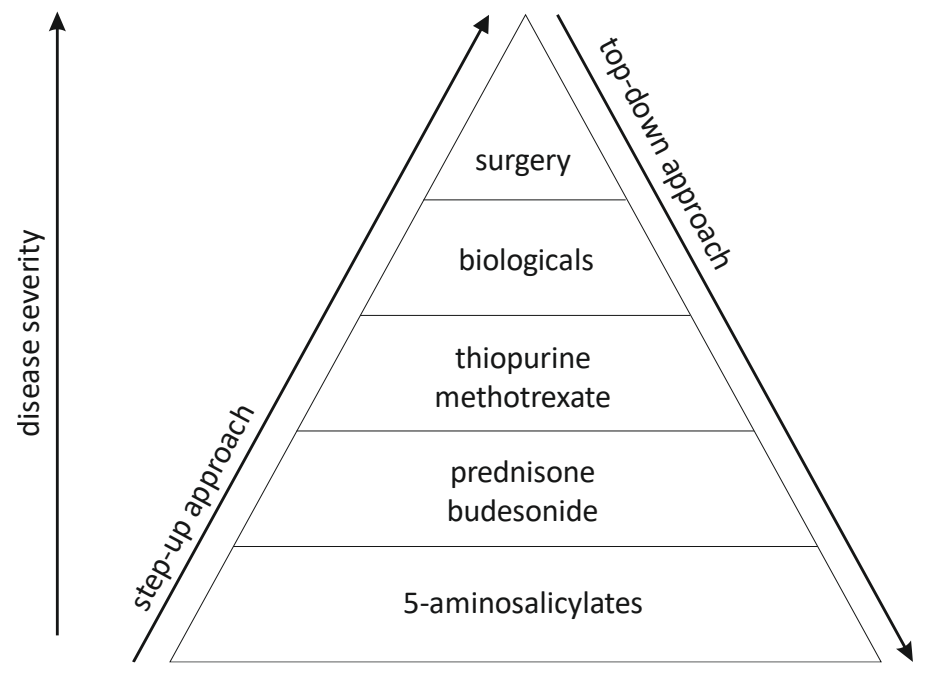

Figure 1.2. Treatment pyramid in inflammatory bowel disease.

The rationale behind the step-up approach is to avert overtreatment, which is important considering the immunosuppressive characteristics of medical therapies used in IBD and the subsequent risks on the short-term [e.g. opportunistic infections] and long-term [e.g. increased risk of lymphomas]. ${ }^{89}$ This approach however prolongs corticosteroid use and delays introduction of potent biological agents in patients with a poor prognosis. An alternative to the previously discussed 'step-up' strategy is the so-called 'accelerated step-up' in which secondline medication is initiated early in the treatment paradigm, based upon prognostic patient or disease characteristics or early signs of failure of the primary therapy. Many propose that a 'top-down' strategy may actually be the best treatment strategy in IBD. ${ }^{90}$ Accordingly, initial treatment consists of a combination of anti-TNF $\alpha$ and immunomodulator therapy [commonly 
referred to as 'combination therapy']. Currently, this strategy is advised for patients with severe disease or who have prognostic factors associated with a disabling disease course. ${ }^{91}$

Both the 'accelerated step-up' and the 'top-down' strategy are characterized by an early aggressive therapeutic approach. The rationale behind this approach comes from the field of rheumatology. Early treatment with disease-modifying antirheumatic drugs was found to be associated with a lower risk of irreversible joint damage on the long-term in rheumatoid arthritis [RA]. ${ }^{92-100}$ Considering the similarity between RA and IBD [i.e. both being immunemediated diseases with structural organ damage on the long-term], it can be expected that employing a similar approach also gives a better long-term outcome in IBD.

\section{New era, better outcome?}

Over the past decades, several changes in the diagnosis and management of IBD were introduced in clinical practice, which may have impacted disease outcome.

First, diagnostic modalities were improved and new modalities became available. For example, full ileocolonoscopic procedures are currently more easily accessible and of much higher quality than in the past, and access to computerized tomography [CT] and magnetic resonance imaging [MRI] has increased, while the associated costs decreased. Furthermore, the transition of IBD care from specialists in internal medicine towards specialized gastroenterologists has probably led to more disease awareness and improved disease control. Furthermore, the development of national and international guidelines has probably led to a more uniform and evidence-based treatment strategy. ${ }^{53-55}$ As a result, it can be assumed that IBD is more readily diagnosed nowadays, and that disease activity and structural bowel damage can be detected more easily.

Second, novel drugs became available and novel treatment strategies have been adopted. As previously discussed, anti-TNF $\alpha$ therapy was introduced for IBD in the late nineties with infliximab being the first ant-TNF $\alpha$ agent registered in the Netherlands [1999 for CD, 2006 for UC], later followed by adalimumab [2007 for CD, 2012 for UC] and golimumab [2013 for UC]. On the other hand, antibiotics and aminosalicylate therapy for ileocecal CD were no longer considered to be effective. ${ }^{54,67}$ Regarding treatment strategy, therapy is sooner being escalated ['accelerated step-up'], reflected by increasing exposure rates of immunomodulators in recent studies. ${ }^{39}$ Finally, the treatment goal has changed from clinical remission to corticosteroid-free remission and even deep remission [i.e. endoscopic or even histological remission]. These therapeutic changes may have led to a better disease control and it is currently unknown whether these have impacted the long-term disease outcome of IBD. 
The relevance of studying medication effectiveness in a real-world population The efficacy of different treatment modalities for IBD has predominantly been studied in randomized controlled trials [RCTs], which deliver the strongest level of evidence, preferably pooled in a systematic review with a meta analysis. ${ }^{101,102}$ RCTs have a strong internal validity, that is, confounding and bias are minimized by using a randomized group allocation and stringent in- and exclusion criteria. As a result, the association between the independent and dependent parameter can be considered causal. Therefore, RCTs show whether study medication has a potential health benefit over placebo, the so-called efficacy of the drug. ${ }^{103}$

It is important to realize that RCTs are performed under strict study conditions and may therefore differ from daily clinical practice. Therefore, their external validity is limited. As a result, the clinical effects of many medical therapies are often lower in real-life clinical practice than in the RCT they were initially studied. The real-life situation often differs from a trial setting in terms of, for example, a more heterogeneous patient population, less stringent disease monitoring and management, and a different drug indication and timing due to drug availability and costs. ${ }^{104}$ Furthermore, in clinical practice, medication is prescribed to subgroups of patients who are often excluded in trials, such as patients with many comorbidities and elderly. All these factors can result in a different 'real-world effect' of the drug, also called the drug's effectiveness. ${ }^{103}$

Pharmacoepidemiology is the field of epidemiology that studies the use and effects of drugs in human populations. ${ }^{105}$ Studying the effectiveness of medical therapies has recently become of increasing interest in $\mathrm{BDD}$, given that $59 \%[\mathrm{UC}]$ and $71 \%[\mathrm{CD}]$ of the current healthcare costs on IBD in the Netherlands are explained by medication use. ${ }^{106}$ Furthermore, pharmacoepidemiological studies may also identify targets for further treatment optimization, for example patient and disease characteristics associated with better response or a beneficial effect of concomitant use with another drug. Head-to-head drug comparisons which were not assessed in an RCT can be conducted in an observational study design, by comparing the realworld effectiveness of the agents, adequately adjusted for bias and confounding. Ultimately, effectiveness research reflects on an important clinical question, useful for health care planning and reimbursement: is the drug's availability of added value for disease management?

\section{Outline of the thesis}

IBD is a heterogeneous, invalidating disease affecting mostly young adults. Its incidence seems increasing worldwide. Over the past decades, the management of IBD has changed markedly. To what extent these changes have resulted in a better outcome for patients in real-world has hardly been studied in representative populations. The high medication costs and broad therapeutic armamentarium further illustrate the need for information on the effectiveness 
and comparative effectiveness of medical therapy in a real-world setting, which can help to avert under- and overtreatment of IBD patients in the future.

In this thesis, temporal changes in the epidemiology and long-term outcome of IBD are studied, with special focus on the role of medical therapy. The basis of this thesis is the population-based IBD South Limburg [IBDSL] cohort; a large population-based inception cohort of well-characterized IBD patients with detailed information on disease characteristics and medication use from patients diagnosed between 1991 and 2011 with a follow-up up to 2014.

In the first part of this thesis, the epidemiology of IBD in South Limburg is described, starting with the rationale and set-up of the IBDSL cohort, presented in Chapter 2. Based on the data of IBDSL, temporal changes in IBD incidence, presenting phenotype and mortality over the past twenty years in the South Limburg area were studied to assess the current disease burden. The findings are presented in Chapter 3. Given the ageing population worldwide, the incidence and disease course of elderly-onset IBD was evaluated separately and directly compared to adult-onset disease. The findings are addressed in Chapter 4.

The second part of this thesis aims to assess whether the outcome of IBD has improved over the past two decades, acknowledging key changes in IBD management. In Chapters 5 and 6 , the long-term outcomes of CD and UC are discussed, respectively. Both studies address changes in medical management and long-term outcome, as assessed in terms of hospitalization risk, surgery risk and the risk of progression to a complicated disease phenotype [CD]. In the CD study, a propensity score analysis was performed to study the direct relation between disease outcome and medication use. In Chapter 7 , we report on the point prevalence of corticosteroid use in a population-based setting and discuss the important treatment goal of corticosteroid sparing. In Chapter 8 , temporal trends in the incidence and outcome of perianal fistulizing CD were studied. In Chapter 9, the long-term real-world effectiveness of anti-TNF $\alpha$ agents in the Netherlands is discussed, with special focus on the question whether infliximab and adalimumab are equally effective. Finally, in Chapter 10, the findings of the thesis are integrated and its implications for the future of IBD management are outlined. 


\section{References}

1. Crohn BB, Ginzburg L, Oppenheimer GD. Regional ileitis: A pathologic and clinical entity. JAMA 1932;99:1323-9.

2. Cosnes J, Gower-Rousseau C, Seksik P, et al.. Epidemiology and natural history of inflammatory bowel diseases. Gastroenterology 2011;140:1785-94.

3. Rieder F, Zimmermann EM, Remzi FH, et al. Crohn's disease complicated by strictures: A systematic review. Gut 2013;62:1072-84.

4. Scharl M, Rogler G. Pathophysiology of fistula formation in Crohn's disease. World J Gastrointest Pathophysiol 2014;5:205-12.

5. Schwartz DA, Loftus EV, Jr., Tremaine WJ, et al. The natural history of fistulizing Crohn's disease in Olmsted county, Minnesota. Gastroenterology 2002;122:875-80.

6. Lennard-Jones JE. Classification of inflammatory bowel disease. Scand J Gastroentero/Suppl 1989;170:2-6; discussion 16-9.

7. Solberg IC, Lygren I, Jahnsen J, et al. Clinical course during the first 10 years of ulcerative colitis: Results from a population-based inception cohort (ibsen study). Scand J Gastroenterol 2009;44:431-40.

8. Solberg IC, Vatn MH, Hoie O, et al. Clinical course in Crohn's disease: Results of a Norwegian populationbased ten-year follow-up study. Clin Gastroenterol Hepatol 2007;5:1430-8.

9. Bernstein CN, Blanchard JF, Rawsthorne P, et al. The prevalence of extraintestinal diseases in inflammatory bowel disease: A population-based study. Am J Gastroentero/ 2001;96:1116-22.

10. Molodecky NA, Soon IS, Rabi DM, et al. Increasing incidence and prevalence of the inflammatory bowel diseases with time, based on systematic review. Gastroenterology 2012;142:46-54 e42; quiz e30.

11. Burisch J, Jess T, Martinato M, Lakatos $\mathrm{PL}$, The burden of inflammatory bowel disease in Europe. J Crohns Colitis 2013;7:322-37.

12. Romberg-Camps MJ, Hesselink-van de Kruijs MA, Schouten LJ, et al. Inflammatory bowel disease in South Limburg (the Netherlands) 1991-2002: Incidence, diagnostic delay, and seasonal variations in onset of symptoms. $J$ Crohns Colitis 2009;3:115-24.

13. Russel MG, Dorant E, Volovics $A$, et al. High incidence of inflammatory bowel disease in the Netherlands: Results of a prospective study. The South Limburg IBD study group. Dis Colon Rectum 1998;41:33-40.

14. Sartor RB. Mechanisms of disease: Pathogenesis of Crohn's disease and ulcerative colitis. Nat. Rev. Gastroenterol. Hepatol. 2006;3:390.

15. Heliö T, Halme L, Lappalainen M, et al. Card15/nod2 gene variants are associated with familially occurring and complicated forms of Crohn's disease. Gut 2003;52:558-62.

16. Hampe J, Grebe J, Nikolaus S, et al. Association of nod2 (card 15) genotype with clinical course of Crohn's disease: A cohort study. Lancet 2002;359:1661-5.

17. Wehkamp J, Harder J, Weichenthal M, et al. NOD2 (CARD15) mutations in Crohn's disease are associated with diminished mucosal $\alpha$-defensin expression. Gut 2004;53:1658-64.

18. de Lange KM, Moutsianas L, Lee JC, et al. Genome-wide association study implicates immune activation of multiple integrin genes in inflammatory bowel disease. Nat. genetics 2017;49:256-61. 
19. Orholm M, Binder V, Sørensen T, et al. Concordance of inflammatory bowel disease among Danish twins: Results of a nationwide study. Scand J Gastroentero/2000;35:1075-81.

20. $\mathrm{Ng} \mathrm{SC}$, Bernstein $\mathrm{CN}$, Vatn $\mathrm{MH}$, et al. Geographical variability and environmental risk factors in inflammatory bowel disease. Gut 2013;62:630-49.

21. Burisch J, Pedersen N, Cukovic-Cavka S, et al. East-west gradient in the incidence of inflammatory bowel disease in Europe: The ECCO-EPICOM inception cohort. Gut 2014;63:588-97.

22. Probert CS, Jayanthi V, Pinder D, et al. Epidemiological study of ulcerative proctocolitis in Indian migrants and the indigenous population of Leicestershire. Gut 1992;33:687-93.

23. Pinsk V, Lemberg DA, Grewal K, et al. Inflammatory bowel disease in the South Asian pediatric population of British Columbia. Am J Gastroenterol 2007;102:1077-83.

24. Barreiro-de Acosta M, Alvarez Castro A, Souto R, et al. Emigration to western industrialized countries: A risk factor for developing inflammatory bowel disease. J Crohns Colitis 2011;5:566-9.

25. Maaser C, Langholz E, Gordon H, et al. European Crohn's and Colitis Organisation topical review on environmental factors in IBD. J Crohns Colitis 2016:jjw223.

26. Benchimol El, Fortinsky KJ, Gozdyra P, et al. Epidemiology of pediatric inflammatory bowel disease: A systematic review of international trends. Inflamm Bowel Dis 2011;17:423-39.

27. Shivananda S, Lennard-Jones J, Logan $\mathrm{R}$, et al. Incidence of inflammatory bowel disease across Europe: Is there a difference between north and south? Results of the European collaborative study on inflammatory bowel disease (EC-IBD). Gut 1996;39:690-7.

28. Linton PJ, Dorshkind K. Age-related changes in lymphocyte development and function. Nat. Immunol. 2004;5:133-9.

29. Min H, Montecino-Rodriguez E, Dorshkind K. Effects of aging on early band t-cell development. Immunol. Rev 2005;205:7-17.

30. Schiffrin EJ, Morley JE, Donnet-Hughes $A$, et al. The inflammatory status of the elderly: The intestinal contribution. Mutat. Res. 2010;690:50-6.

31. Magrone T, Jirillo E. The interaction between gut microbiota and age-related changes in immune function and inflammation. Immun Ageing 2013;10.

32. Boyko EJ, Koepsell TD, Perera DR, et al. Risk of ulcerative colitis among former and current cigarette smokers. N Eng/ J Med 1987;316:707-10

33. Mahid SS, Minor KS, Soto RE, et al. Smoking and inflammatory bowel disease: A meta-analysis. Mayo Clin Proc 2006;81:1462-71

34. Cosnes J. Tobacco and IBD: Relevance in the understanding of disease mechanisms and clinical practice. Best Pract Res Clin Gastroenterol 2004;18:481-96.

35. UnitedNations. World population ageing 2009. New York: United Nations publication; 2010.

36. Faubion WA, Jr., Loftus EV, Jr., Harmsen WS, et al. The natural history of corticosteroid therapy for inflammatory bowel disease: A population-based study. Gastroenterology 2001;121:255-60.

37. Silverstein MD, Loftus EV, Sandborn WJ, et al. Clinical course and costs of care for Crohn's disease: Markov model analysis of a population-based cohort. Gastroenterology 1999;117:49-57.

38. Rungoe $\mathrm{C}$, Langholz E, Andersson M, et al. Changes in medical treatment and surgery rates in inflammatory bowel 
disease: A nationwide cohort study 1979-2011. Gut 2014;63:1607-16.

39. Ramadas AV, Gunesh S, Thomas GA, et al. Natural history of Crohn's disease in a population-based cohort from Cardiff (1986-2003): A study of changes in medical treatment and surgical resection rates. Gut 2010;59:1200-6.

40. Vester-Andersen MK, Prosberg MV, Jess

$\mathrm{T}$, et al. Disease course and surgery rates in inflammatory bowel disease: A population-based, 7-year follow-up study in the era of immunomodulating therapy. Am J Gastroentero/ 2014;109:705-14.

41. Lakatos PL, Golovics PA, David G, et al. Has there been a change in the natural history of Crohn's disease? Surgical rates and medical management in a population-based inception cohort from Western Hungary between 1977-2009. Am J Gastroentero/ 2012;107:579-88.

42. Charpentier C, Salleron J, Savoye G, et al. Natural history of elderly-onset inflammatory bowel disease: A population-based cohort study. Gut 2014;63:423-32.

43. Hoie $\mathrm{O}$, Wolters F, Riis L, et al. Ulcerative colitis: Patient characteristics may predict 10-yr disease recurrence in a European-wide population-based cohort. Am J Gastroentero/ 2007; 102:1692701.

44. Hoie O, Wolters FL, Riis L, et al. Low colectomy rates in ulcerative colitis in an unselected European cohort followed for 10 years. Gastroenterology 2007:132:507-15.

45. Jess $T$, Riis $L$, Vind I, et al. Changes in clinical characteristics, course, and prognosis of inflammatory bowel disease during the last 5 decades: A populationbased study from Copenhagen, Denmark. Inflamm Bowel Dis 2007:13:481-9.

46. Kaplan GG, Seow CH, Ghosh S, et al. Decreasing colectomy rates for ulcerative colitis: A population-based time trend study. Am J Gastroenterol 2012;107:1879-87.

47. Magro F, Rodrigues A, Vieira Al, et al. Review of the disease course among adult ulcerative colitis population-based longitudinal cohorts. Inflamm Bowel Dis 2012;18:573-83.

48. Targownik LE, Singh $H$, Nugent $Z$, et al. The epidemiology of colectomy in ulcerative colitis: Results from a population-based cohort. Am J Gastroenterology 2012;107:1228-35.

49. Wolters FL, Russel MG, Sijbrandij J, et al. Phenotype at diagnosis predicts recurrence rates in Crohn's disease. Gut 2006:55:1124-30.

50. Bernstein CN, Loftus EV, Ng SC, et al. Hospitalisations and surgery in Crohn's disease. Gut 2012:-2011-301397.

51. Lakatos PL, David G, Pandur T, et al. IBD in the elderly population: Results from a population-based study in Western Hungary, 1977-2008. J Crohns Colitis 2011;5:5-13.

52. Cosnes J, Cattan S, Blain A, et al. Longterm evolution of disease behavior of crohn's disease. Inflamm Bowel Dis 2002:8:244-50.

53. Dignass A, Lindsay JO, Sturm A, et al. Second European evidence-based consensus on the diagnosis and management of ulcerative colitis part 2: Current management. J Crohns Colitis 2012;6:991-1030.

54. Dignass A, Van Assche G, Lindsay JO, et al. The second European evidence-based consensus on the diagnosis and management of Crohn's disease: Current management. J Crohns Colitis 2010;4:28-62.

55. van Bodegraven AA, van Everdingen $\mathrm{JJ}$, Dijkstra G, et al. [guideline 'diagnosis and treatment of inflammatory bowel disease in adults'. I. Diagnosis and treatment]. Ned Tijdschr Geneeskd 2010;154:A1899. 
56. Hayashi $\mathrm{R}$, Wada $\mathrm{H}$, Ito $\mathrm{K}$, et al. Effects of glucocorticoids on gene transcription. Eur J Pharmacol 2004;500:51-62.

57. Summers RW, Switz DM, Sessions JT, Jr., et al. National cooperative Crohn's disease study: Results of drug treatment. Gastroenterology 1979;77:847-69.

58. Steinhart AH, Ewe K, Griffiths AM, et al. Corticosteroids for maintenance of remission in Crohn's disease. Cochrane 2003:Cd000301.

59. Curtis JR, Westfall AO, Allison J, et al. Population-based assessment of adverse events associated with long-term glucocorticoid use. Arthritis Rheum 2006;55:420-6.

60. Hoes JN, Jacobs JW, Verstappen SM, et al. Adverse events of low- to mediumdose oral glucocorticoids in inflammatory diseases: A meta-analysis. Ann Rheum Dis 2009;68:1833-8.

61. Rousseaux C, Lefebvre B, Dubuquoy L, et al. Intestinal antiinflammatory effect of 5-aminosalicylic acid is dependent on peroxisome proliferator-activated receptor-. . J. Exp. Med. 2005;201:1205-15.

62. Shanahan F, Niederlehner A, Carramanzana N, Anton P. Sulfasalazine inhibits the binding of TNF $\alpha$ to its receptor. Immunopharmacology 1990;20:217-24.

63. Berg C, Bantel H, Schulze-Osthoff $K$, et al. Mesalazine inhibits activation of transcription factor NF- $\mathrm{k} \beta$ in inflamed mucosa of patients with ulcerative colitis. Am J Gastroenterol 2000;95:3452.

64. Ahnfelt-Ronne I, Nielsen OH, Christensen A, et al. Clinical evidence supporting the radical scavenger mechanism of 5aminosalicylic acid. Gastroenterology 1990;98:1162-9.

65. Stevens C, Lipman M, Fabry S, et al. 5aminosalicylic acid abrogates T-cell proliferation by blocking interleukin-2 production in peripheral blood mononuclear cells. J. Pharmacol. Exp. Ther. 1995;272:399-406.

66. Neal TM, Winterbourn CC, Vissers MC Inhibition of neutrophil degranulation and superoxide production by sulfasalazine: Comparison with 5-aminosalicylic acid, sulfapyridine and olsalazine. Biochem. Pharmacol. 1987;36:2765-8.

67. Hanauer SB, Strömberg U. Oral pentasa in the treatment of active Crohn's disease: A meta-analysis of double-blind, placebo-controlled trials. Clin. Gastroenterol. Hepatol. 2004;2:379-88.

68. Willoughby JM, Beckett J, Kumar PJ, et al. Controlled trial of azathioprine in Crohn's disease. Lancet 1971;2:944-7.

69. Elion GB. The purine path to chemotherapy. Portland Press Limited, 1989.

70. Poppe D, Tiede I, Fritz G, et al. Azathioprine suppresses ezrin-radixinmoesin-dependent T cell-APC conjugation through inhibition of vav guanosine exchange activity on $\mathrm{RaC}$ proteins. J. Immunol. 2006;176:640-51.

71. Trotter J, Rodey G, Gebel H. Azathioprine decreases suppressor $T$ cells in patients with multiple sclerosis. $N$ Eng/ J Med 1982;306:365.

72. Ten Hove T, Van Montfrans C, Peppelenbosch $\mathrm{M}$, et al.. Infliximab treatment induces apoptosis of lamina propria T lymphocytes in Crohn's disease. Gut 2002;50:206-11.

73. Levin AD, Wildenberg ME, van den Brink GR. Mechanism of action of anti-TNF therapy in inflammatory bowel disease. $J$ Crohns Colitis 2016;10:989-97.

74. Targan SR, Hanauer SB, van Deventer SJ, et al. A short-term study of chimeric monoclonal antibody $\mathrm{Ca} 2$ to tumor necrosis factor alpha for Crohn's disease. Crohn's disease Ca2 study group. N Eng/ J Med 1997;337:102935.

75. Hanauer SB, Feagan BG, Lichtenstein $G R$, et al. Maintenance infliximab for 
Crohn's disease: The ACCENT I

randomised trial. Lancet

2002;359:1541-9.

76. Rutgeerts P, Sandborn WJ, Feagan BG, et al. Infliximab for induction and maintenance therapy for ulcerative colitis. N Eng/ J Med 2005;353:246276.

77. Reinisch W, Sandborn WJ, Rutgeerts P, et al. Long-term infliximab maintenance therapy for ulcerative colitis: The ACT-1 and -2 extension studies. Inflamm Bowel Dis 2012;18:201-11.

78. Colombel JF, Sandborn WJ, Rutgeerts P, et al. Adalimumab for maintenance of clinical response and remission in patients with Crohn's disease: The charm trial. Gastroenterology 2007;132:52-65.

79. Hanauer SB, Sandborn WJ, Rutgeerts P, et al. Human anti-tumor necrosis factor monoclonal antibody (adalimumab) in Crohn's disease: The CLASSIC-I trial. Gastroenterology 2006;130:323-33; quiz 591.

80. Sandborn WJ, van Assche G, Reinisch W, et al. Adalimumab induces and maintains clinical remission in patients with moderate-to-severe ulcerative colitis. Gastroenterology 2012;142:257-65 e1-3.

81. Sandborn WJ, Feagan BG, Marano C, et al. Subcutaneous golimumab maintains clinical response in patients with moderate-to-severe ulcerative colitis. Gastroenterology 2014;146:96-109. e1.

82. Sandborn WJ, Feagan BG, Marano C, et al. Subcutaneous golimumab induces clinical response and remission in patients with moderate-to-severe ulcerative colitis. Gastroenterology 2014;146:85-95.

83. Danese S, Gomollon F, Governing B, Operational Board of E. ECCO position statement: The use of biosimilar medicines in the treatment of inflammatory bowel disease (IBD). $J$ Crohns Colitis 2013;7:586-9.

84. Jha A, Upton A, Dunlop WC, Akehurst R. The budget impact of biosimilar infliximab (Remsima) for the treatment of autoimmune diseases in five European countries. Adv Ther 2015;32:742-56.

85. Sandborn WJ, Feagan BG, Rutgeerts $P$, et al. Vedolizumab as induction and maintenance therapy for Crohn's disease. N Eng/ J Med2013;369:71121.

86. Feagan BG, Greenberg GR, Wild G, et al. Treatment of ulcerative colitis with a humanized antibody to the $\alpha 4 \beta 7$ integrin. N Eng/ J Med 2005;352:2499507.

87. Wyant T, Fedyk E, Abhyankar B. An overview of the mechanism of action of the monoclonal antibody vedolizumab. J Crohns Colitis 2016;10:1437-44.

88. Feagan BG, Sandborn WJ, Gasink C, et al. Ustekinumab as induction and maintenance therapy for Crohn's disease. N Eng/ J Med 2016;375:1946-60.

89. Mason M, Siegel CA. Do inflammatory bowel disease therapies cause cancer? Inflamm Bowel Dis 2013;19:1306-21.

90. D'Haens G, Baert F, van Assche G, et al. Early combined immunosuppression or conventional management in patients with newly diagnosed Crohn's disease: An open randomised trial. Lancet 2008;371:660-7.

91. Gomollon F, Dignass A, Annese V, et al. 3rd European evidence-based consensus on the diagnosis and management of Crohn's disease 2016: Part 1: Diagnosis and medical management. J Crohns Colitis 2017;11:3-25.

92. Bukhari M, Wiles $\mathrm{N}$, Lunt $\mathrm{M}$, et al. Influence of disease-modifying therapy on radiographic outcome in inflammatory polyarthritis at five years: 
Results from a large observational inception study. Arthritis Rheumatol. 2003;48:46-53.

93. Egsmose C, Lund B, Borg G, et al. Patients with rheumatoid arthritis benefit from early 2nd line therapy: 5 year followup of a prospective double blind placebo controlled study. J Rheumatol 1995:22:2208-13.

94. Lard LR, Visser H, Speyer I, et al. Early versus delayed treatment in patients with recent-onset rheumatoid arthritis: Comparison of two cohorts who received different treatment strategies. Am. J. Med. 2001;111:446-51.

95. Möttönen T, Hannonen P, Korpela M, et al. Delay to institution of therapy and induction of remission using single-drug or combination-disease-modifying antirheumatic drug therapy in early rheumatoid arthritis. Arthritis Rheumatol: 2002;46:894-8.

96. van der Heide A, Jacobs JW, Bijlsma JW, et al. The effectiveness of early treatment with "second-line" antirheumatic drugs a randomized, controlled trial. Ann. Intern. Med. 1996;124:699-707.

97. Fries JF, Williams CA, Morfeld D, et al. Reduction in long-term disability in patients with rheumatoid arthritis by disease-modifying antirheumatic drugbased treatment strategies. Arthritis Rheumatol 1996;39:616-22.

98. Sokka T, Möttönen T, Hannonen P. Disease-modifying anti-rheumatic drug use according to the sawtooth treatment strategy improves the functional outcome in rheumatoid arthritis: Results of a long-term follow-up study with review of the literature. Rheumatology 2000;39:34-42.

99. Breedveld FC, Weisman MH, Kavanaugh $A F$, et al. The premier study: A multicenter, randomized, double-blind clinical trial of combination therapy with adalimumab plus methotrexate versus methotrexate alone or adalimumab alone in patients with early, aggressive rheumatoid arthritis who had not had previous methotrexate treatment. Arthritis Rheumatol 2006;54:26-37.

100. Goekoop-Ruiterman YP, de VriesBouwstra JK, Allaart CF, et al. Clinical and radiographic outcomes of four different treatment strategies in patients with early rheumatoid arthritis (the best study): A randomized, controlled trial. Arthritis Rheumatol 2005;52:3381-90.

101. Group OLoEW. " The Oxford 2011 levels of evidence." Oxford Centre for evidence-based medicine. http://www.cebmnet/indexaspx?o=56 532011.

102. Concato J, Shah N, Horwitz Rl. Randomized, controlled trials, observational studies, and the hierarchy of research designs. N Eng/ J Med 2000;342:1887-92.

103. Salleron J, Danese S, D'Agay L, et al. Effectiveness research in inflammatory bowel disease: A necessity and a methodological challenge. J Crohns Colitis 2016;10:1096-102.

104. Ha C, Ullman TA, Siegel CA, et al. Patients enrolled in randomized controlled trials do not represent the inflammatory bowel disease patient population. Clin Gastroenterol Hepatol 2012;10:1002-7; quiz e78.

105. Yang $Y$, West-Strum D. Understanding pharmacoepidemiology. 2011.

106. Van Der Valk ME, Mangen M-JJ, Leenders M, et al. Healthcare costs of inflammatory bowel disease have shifted from hospitalisation and surgery towards anti-TNF $\alpha$ therapy: Results from the coin study. Gut 2014;63:72-9. 



\section{PART I}

Epidemiology of

inflammatory bowel disease

in South Limburg 



\section{CHAPTER 2}

\section{Cohort Profile:}

The inflammatory bowel disease South Limburg Cohort [IBDSL]

Tim RA van den Heuvel, Daisy MAE Jonkers, Steven FG Jeuring, Marielle JL Romberg-Camps, Liekele E Oostenbrug, Maurice P Zeegers, Ad AM Masclee, Marieke J Pierik

Int J Epidemiol. 2017 Apr 1;46(2):e7 


\section{Abstract}

The inflammatory bowel disease South Limburg [IBDSL] cohort is designed as a Dutch population-based cohort study on inflammatory bowel disease [IBD] and has been used to study its epidemiology and disease course since 1991. Every newly diagnosed IBD patient, above 18 years of age and living in the South Limburg area at time of diagnosis, is eligible. By using a multifaceted approach, we were able to identify over $93 \%$ of the eligible IBD population and followed them prospectively. Currently, 2837 IBD patients are included, of which 1162 have Crohn's disease [CD] and 1675 ulcerative colitis [UC]. Demographic and clinical data were assessed at baseline [i.e. disease phenotype, extra intestinal manifestations, complications, medication use, surgery status, and pathology-, endoscopy- and radiology reports] and data have been updated biennially through chart review, questionnaires, and linkage to the Dutch resident registration. As of 2011, IBDSL is being scaled up into a crosssectional, population-based biobank, and focus expanded towards exploring underlying biologic mechanisms and molecular epidemiology. The biobank currently covers 957 patients [535 CD, 422 UC] and includes serum, plasma, DNA and feces. In addition, biopsies and exhaled air are also collected from a subset. We welcome new collaborations. 


\section{Why was the cohort set up?}

Inflammatory bowel disease [IBD], encompassing Crohn's disease [CD] and ulcerative colitis [UC], is a chronic inflammatory condition of the intestines, which may have severe impact on patients' quality of life. ${ }^{1-3} \mathrm{IBD}$ is characterized by sequences of exacerbation and remission, and is considered to arise from complex interactions between an altered intestinal immune response, the intestinal microbiota and environmental factors in a genetically susceptible host. ${ }^{4}$ Its presentation is heterogeneous, and also treatment response varies. In the IBD field, identification and validation of biomarkers to predict disease course and therapy response is currently an important challenge. Also, the further unravelling of [biological] disease mechanisms remains of paramount importance, especially with regard to the development of exacerbations.

The inflammatory bowel disease South Limburg [IBDSL] cohort is a long-term, ongoing, population-based cohort with deep phenotyping and clinical data, and an extensive biomaterial collection [referred to as the IBDSL biobank]. The IBDSL cohort started in 1991 as part of the European Collaborative study on Inflammatory Bowel Disease [EC-IBD]. ${ }^{5}$ Twenty centers throughout Europe began registering all newly diagnosed patients in order to study a hypothesized North-South gradient in IBD incidence. IBDSL proceeded with registering patients and their clinical data, and has been studying IBD epidemiology, disease course, risk factors for disease onset and development, and quality of life, ever since. As of 2011, the cohort is being scaled up into a population-based biobank and the focus expanded towards exploring underlying biological disease mechanisms and molecular epidemiology. Populationbased biomaterial collections, representing the full heterogeneous IBD spectrum [from mild to severely diseased], are well equipped for this research and warranted.

IBDSL is a Dutch consortium comprising the gastroenterology departments of Maastricht University Medical Center+ [MUMC+] and the general district hospitals Orbis MC Sittard-Geleen and Atrium MC Heerlen. IBDSL has been approved by the Ethics Committee of the Maastricht University Medical Center+ [NL31636.068.10], meets the ethical standards of the revised version of the Declaration of Helsinki, and is registered at www.ClinicalTrial.gov [NCT02130349]. 


\section{Who is in the cohort?}

\section{Design}

The IBDSL cohort is a long-term, population-based, prospective, inception IBD cohort with a cross-sectional biobank.

\section{Setting}

South Limburg is a geographical area in the southeast of the Netherlands, bordered by Belgium and Germany [Figure 2.1]. In January 2014, South Limburg had 604,154 inhabitants. ${ }^{6}$ Apart from the general district hospitals of Heerlen and Sittard-Geleen, the Maastricht University Medical Center+ and general practitioners, no other in- or outpatient clinics are entrusted with IBD care in this area. As cross-border health care is limited and migration rates are rather low, ${ }^{6}$ South Limburg provides an ideal setting for population-based research. All IBD patients, diagnosed above 18 years of age and living in South Limburg at time of diagnosis, are eligible. IBD is diagnosed by certified gastroenterologists according to the Lennard-Jones criteria ${ }^{7}$ and proven by endoscopic, radiological and/or histological findings.

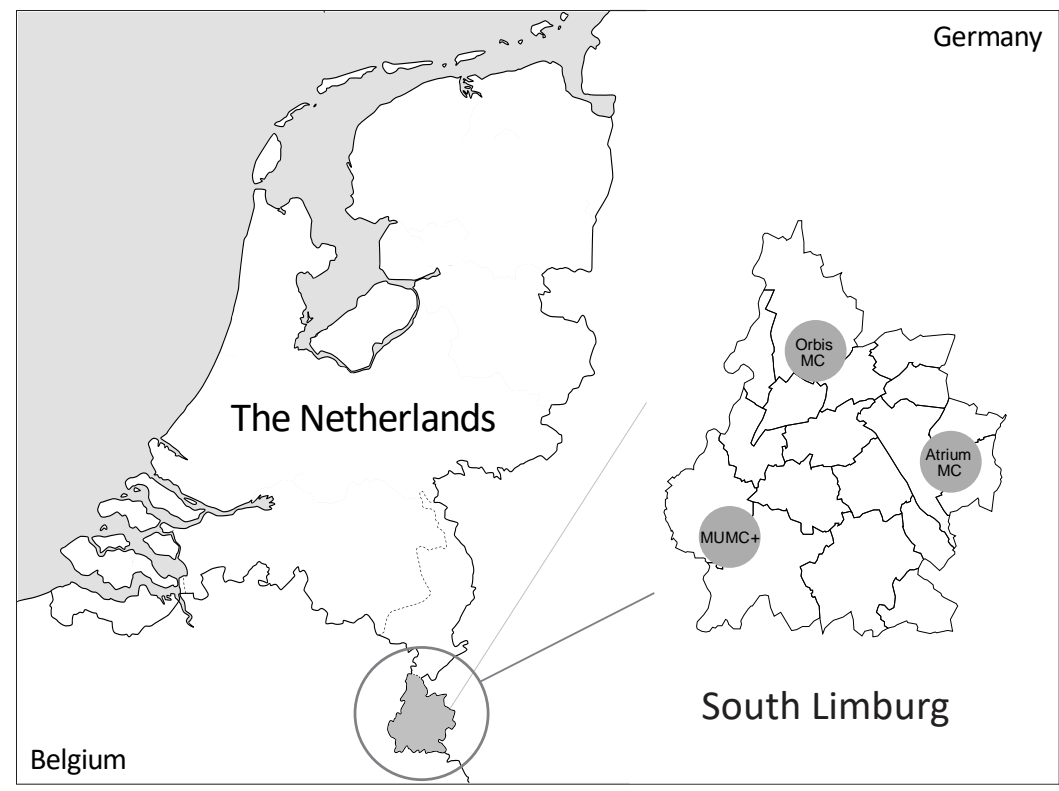

Figure 2.1. Geographic location of South Limburg, including the IBDSL consortium centers: Maastricht University Medical Center+ [MUMC+], Atrium Medical Center Heerlen [Atrium MC] and Orbis Medical Center Sittard-Geleen [Orbis MC] 


\section{IBDSL cohort study population}

To capture the full South Limburg IBD population, we use a multifaceted approach. First, newly diagnosed patients are registered prospectively by the gastroenterologists and IBD nurses of the participating hospitals. Second, any missed patients are identified through regular hospital administration checks. Finally, we cooperate with PALGA, a histopathology registry covering all pathology reports generated in the Netherlands [since 1991], to identify IBD patients who do not attend hospitals.

In Table 2.1 an overview is shown of the IBDSL cohort study population [with minimum follow-up of 2 years]. In January 2014, this comprised 2837 IBD patients, of whom 1162 were diagnosed with $C D$ and 1675 with UC. The study population represents over 93\% of all eligible patients in South Limburg, determined by comparing IBDSL cases with IBD cases of a representative sample of 18 South Limburg general practitioners [GP]. In the Netherlands, GPs are the first to be contacted for health care. Therefore, IBD patients in referral care are also known in GP registries, which make them suitable for assessing completeness.

The remaining $7 \%$ could not be captured for ethical and logistic reasons. Hence, we were not able to compare IBD phenotypes between included and missed patients [i.e. comparison according to the Montreal classification ${ }^{9}$ which considers age of onset, disease location/extent and behavior]. Selection bias, however, is not expected; phenotypes that can easily be missed in non-population-based cohorts, such as mildly diseased patients not attending hospitals, are captured via PALGA. The remaining $7 \%$ of patients mostly likely have an IBD diagnosis without endoscopic, radiological and/or histological confirmation [and do not meet IBDSL inclusion criteria], may be misdiagnosed [e.g. irritable bowel syndrome rather than IBD] or attend Belgian or German hospitals. As guidelines in these countries are similar to those in the Netherlands, as well as costs [cross-border health care is also covered by insurers], it is highly unlikely that cross-border health care is associated with a specific IBD phenotype. 
Table 2.1. Baseline characteristics of the IBDSL cohort study population with minimum 2-year follow-up in January 2014 and characteristics of patients that were lost-to-follow-up

\begin{tabular}{llll}
\hline Baseline & & UC & CD \\
\hline Patients & $N$ & 1675 & 1162 \\
Age at diagnosis & mean in years [SD] & $46.2[16.8]$ & $37.7[15.9]$ \\
Disease duration & mean in years [SD] & $9.1[5.9]$ & $8.4[5.9]$ \\
Males & $N[\%]$ & $895[53]$ & $434[37]$ \\
E1/E2/E3 & $N[\%]$ & $568[34] / 791[48] / 299[18]$ & - \\
L1/L2/ & $N[\%]$ & - & $500[43] / 371[32] /$ \\
L3/L4 & & & $267[23] / 24[2]$ \\
B1/B2/B3 & $N[\%]$ & - & $900[77] / 177[15] / 85[7]$ \\
\hline Follow-up & & UC & CD \\
\hline Death & $N$ & 202 & 77 \\
Males & $N[\%]$ & $130[64]^{b}$ & $29[38]$ \\
E1/E2/E3 & $N[\%]$ & $44[22] / 118[60] / 35[18]^{b}$ & - \\
L1/L2/ & $N[\%]$ & - & $32[42] / 33[43] /$ \\
L3/L4 & & & $10[13] / 2[3]$ \\
B1/B2/B3 & $N[\%]$ & $N$ & $64[83] / 5[6] / 8[10]$ \\
Migrated & $N$ & 101 & 51 \\
Males & $N[\%]$ & $58[57]$ & $23[45]$ \\
E1/E2/E3 & $N[\%]$ & $32[32] / 47[47] / 21[21]$ & - \\
L1/L2/ & $N[\%]$ & - & $22[43] / 18[35]$ \\
L3/L4 & & - & $10[20] / 1[2]$ \\
B1/B2/B3 & $N[\%]$ & $-40[78] / 7[14] / 4[8]$ \\
\hline
\end{tabular}

UC, ulcerative colitis; CD, Crohn's disease; N, number of patients; SD, standard deviation.

a phenotype at diagnosis according to Montreal Classification. Disease extent of UC was defined as: ulcerative proctitis [E1, limited to the rectum], left sided UC [E2, limited to a proportion of the colorectum distal to the splenic flexure] and extensive UC [E3, proximal to the splenic flexure]. CD location was defined as: ileal involvement [L1], exclusive colonic involvement [L2], ileocolonic involvement [L3] or isolated upper disease [L4]. CD behavior was defined as: non-stricturing and non-penetrating [B1], stricturing [B2] or penetrating [B3].

${ }^{b}$ significant difference $[p<0.01]$ in a chi-square analysis of deceased vs living patients $[\alpha=0.05]$

\section{IBDSL biobank study population}

All patients in the IBDSL cohort are also eligible for biomaterial donation. An overview of the biobank study population is shown in Table 2.2. In January 2014, 957 IBD patients were biobanked, of whom 535 had CD and 422 had UC. At this moment, the biobank is still in progress of becoming population based. Donation response rates are high among all phenotypes and based thereupon we expect to biobank approximately $75 \%$ of all patients in the cohort. 
Table 2.2. Characteristics of biomaterial collected in the IBDSL biobank, until January 2014

\begin{tabular}{cccc}
\hline & & UC & CD \\
\hline Biomaterial & $N$ & 422 & 535 \\
DNA & $N$ & 390 & 463 \\
Plasma & $N$ & 390 & 463 \\
Serum & $N$ & 414 & 524 \\
Feces & $N$ & 327 & 405 \\
\hline
\end{tabular}

UC, ulcerative colitis; CD, Crohn's disease; N, number of patients.

\section{How often have they been followed up?}

\section{IBDSL cohort}

Patient registration began in January 1991 and has been embedded in daily clinical practice. Gastroenterologists and IBD nurses completed a standardized registration form for any newly diagnosed patient, and clinical and demographic data at time of diagnosis were assessed. For patients captured through hospital administration or PALGA, data [at time of diagnoses] were assessed retrospectively, immediately after case identification. Clinical and demographic data were updated biennially until loss to follow-up [i.e. death or migration outside the region]. Patient identification and follow-up is an ongoing process.

In Table 2.1, characteristics of loss to follow-up are described. Loss to follow-up by either death or migration was evenly distributed among gender and phenotypes in CD. In UC, loss to follow-up by death was more pronounced in male patients and in patients with leftsided colitis at diagnosis. Such higher proportions of males and left-sided inflammation were also found in our elderly-onset UC population [> 60 years], when compared with adult-onset $\left[<60\right.$ years]. ${ }^{10}$ A shorter life expectancy of the former may lead to the observed skewness in loss to follow-up by death.

\section{IBDSL biobank}

Biomaterial collection began in 2011, using a cross-sectional approach. Biomaterial collection has also been embedded in daily clinical practice; after consulting their gastroenterologist or IBD nurse, patients were requested to participate in biomaterial collection. Patients not attending the hospital regularly were either visited at home or invited to the hospital. In addition, a subgroup of IBD patients were invited for repeated sampling, and they donated biomaterial at every hospital visit for 2 years. We have permission to contact biobanked patients for additional biomaterial donation in future. 


\section{What has been measured?}

An overview of collected data and biomaterials is shown in Table 2.3.

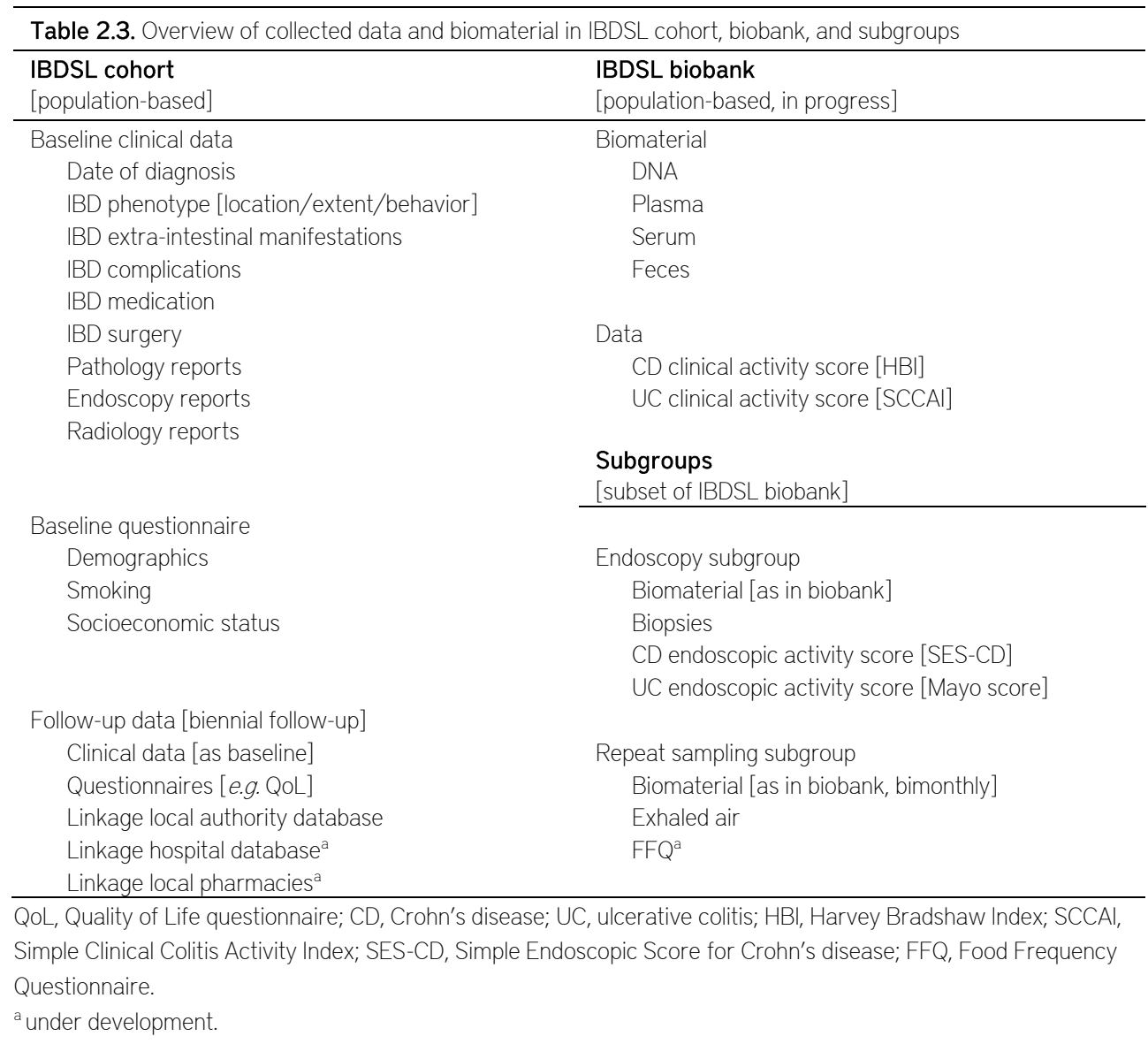

\section{IBDSL cohort}

Baseline data were collected for time of diagnosis, which was set as the date of endoscopic or radiological examination with first evidence for IBD. Case report forms [CRF] were completed and comprised phenotype [Montreal classification, i.e. age of onset, disease location/extent, behavior], extra-intestinal manifestations [eyes, mouth, skin, liver, joints], IBD complications [stenosis, fistula, abscess, thrombosis, perforation, osteoporosis, osteopenia], IBD medication [type, dosage and duration], IBD surgery [type and date], pathology reports, endoscopy reports and radiology reports. For CD patients, disease location at diagnosis was only set after 1 year, as many patients did not have a complete diagnostic workup at time of 
diagnosis. In addition, data on demographics, smoking and socioeconomic status were collected from all patients.

Biennially, clinical and demographic data were updated through chart review and questionnaires. Patients' charts were reviewed by a trained nurse, and CRFs similar to those described above were completed. Questionnaires always comprised quality of life [i.e. Short Inflammatory Bowel Disease Questionnaire [S-IBDQ], Short Form 36 health survey [SF-36]], in addition to different topics each time such as medication adherence, subfertility, fatigue and stress. IBDSL is also linked to the Dutch resident registration for updates on addresses and vital status.

\section{IBDSL biobank}

Biomaterial was collected using standardized operating procedures, and comprised one serum tube, one plasma tube and one feces tube. At the same time, clinical disease activity was determined by the Harvey Bradshaw Index for $C^{11}$ and the Simple Clinical Colitis Activity Index for UC. ${ }^{12}$ Serum tubes [8.5 ml, BD, Plymouth, UK] were centrifuged within $4 \mathrm{~h}$ after collection [3000 G, $10 \mathrm{~min}, 4^{\circ} \mathrm{C}$, without brake]. Plasma tubes [10.0 ml, BD, Plymouth, UK] were also centrifuged within $4 \mathrm{~h}$ after collection [1800 G, $10 \mathrm{~min}$, room temperature [RT], with brake]. DNA was isolated from the buffy coat using the FlexiGene DNA Kit [Qiagen, Venlo, the Netherlands]. After processing, all aliquots were stored at $-80 \mathrm{C}$ in the certified MUMC+ biobank. ${ }^{13}$

\section{IBDSL biobank subgroups}

The IBDSL biobank comprises two subgroups, in which additional biomaterial was collected. Consecutive patients who were scheduled for routine endoscopy and consented to donate biopsies, took part in the 'endoscopy subgroup' [ $n=104]$. A maximum of six biopsies were collected [two terminal ileum, two ascending colon, two descending colon, preferably inflamed tissue], in addition to an endoscopic disease activity score [Simple Endoscopic Activity Score for $\mathrm{CD}^{14}$ and Mayo endoscopic subscore for $\mathrm{UC}^{15}$ ] and the previously described set of serum, plasma, feces and clinical activity scores. Biopsies were collected in a cryogenic vial $[1.5 \mathrm{ml}$, Thermo Scientific Nalgene, Rochester, USA], snap-frozen in liquid nitrogen and stored at $80^{\circ} \mathrm{C}$. This subgroup can be used as a reference cohort for validation purposes, since intestinal inflammation was determined by the gold standard [endoscopy]. A second group of patients were invited for repeat sampling. In this 'repeat sampling subgroup' [n=323] patients donated serum, plasma and feces at every hospital visit for 1 year. These patients also donated exhaled air for volatile organic compounds [VOC] metabolomics. Each patient was asked to inflate a 5-I Tedlar bag [SKC Ltd, Dorset, UK]. Captured VOCs were transferred to carbon-filled sorption tubes [Markes International Ltd, Llantrisant Business Park, UK] and trapped within $2 \mathrm{~h}$ after collection. As this subgroup 
comprises patients going from active disease into remission, and vice versa, it enables testing biomarker consistency over time and in different stages of the disease.

\section{Future}

Several plans to improve IBDSL are scheduled. First, direct linkage of the IBDSL cohort to the hospital databases is currently under development, which will result in an immediate cohort update every time a specialist adds information to the medical file. This update also includes laboratory analyses. Second, linkage to local pharmacies has been initiated. Third, patients with an IBD diagnosis during childhood are not captured at this moment, just as IBD-undefined patients [IBD-U, IBD patients in which a clear distinction between UC and CD cannot be made]. Current efforts are being made to expand IBDSL in order to include these patients. We also started a new study to expand the repeat sampling subgroup with dietary data such as food frequency questionnaires [FFQ] at baseline. Finally, we have permission to include a control group of patients' partners and family members, who will also be invited to donate biomaterial.

\section{What has it found? Key findings and publications}

During its existence, IBDSL has participated in several international consortia. IBDSL was a founding member of the EC-IBD, which studied IBD epidemiology in Europe. ${ }^{5}$ Nowadays, IBDSL is involved in three international consortia [Sysmed-IBD ${ }^{16}, \mid \mathrm{BD}-\mathrm{BIOM}^{17}$ and IBDCharacter ${ }^{18}$, which are funded by the European Commission's Seventh Framework Programme and focus on the identification and validation of biomarkers. Altogether, 55 scientific papers on IBDSL data have been published so far, either autonomously or as part of the consortia. ${ }^{15,19-71}$ Below, a short overview is provided of some previous findings, as well as some recent findings which are currently submitted for publication.

\section{IBDSL cohort}

Early IBDSL cohort studies focused on epidemiology, risk factors for disease onset and progression, and quality of life. IBDSL, for instance, has provided reliable incidence data on IBD in the Netherlands. ${ }^{21,23}$ Between 1991 and 2002, age-standardized incidence rates for CD were 4.84 [per 100,000 person-years] in males and 7.58 in females, and 8.51 and 6.92 for UC, respectively. ${ }^{21}$ These incidences were found to be relatively high compared with other European countries, ${ }^{21,23}$ and contributed to a North-South gradient in European IBD incidence. ${ }^{68}$ We also found age at diagnosis, ${ }^{20}$ phenotype at diagnosis ${ }^{20}$ and smoking ${ }^{25}$ to be predictive for a more severe disease course [the latter only in CD]. IBDSL patients often experienced an impaired health-related quality of life as well as fatigue problems [also when 
in remission] ${ }^{1}$ and, despite a normal life expectancy, ${ }^{19}$ they encountered difficulties when applying for life insurance. ${ }^{28}$

Recent studies concerned long-term disease outcome and disease course variations between patients diagnosed in different time periods. In both UC and CD, for instance, longterm disease outcome of elderly-onset IBD [> 60 years] was similar to adult-onset [<60 years]. ${ }^{10}$ However, less frequent use of immunomodulating drugs and biologicals [anti-tumor necrosis factor [anti-TNF $\alpha$ ] drugs] was observed in the former group. Furthermore, early colectomy rate was found to be 2.4-fold lower in UC patients diagnosed after 2006, the year biologicals were first registered for UC in the Netherlands, as compared with patients diagnosed in the era before biological availability $[<2006] .^{72}$ These colectomies seemed to be prevented rather than postponed [Figure 2.2].

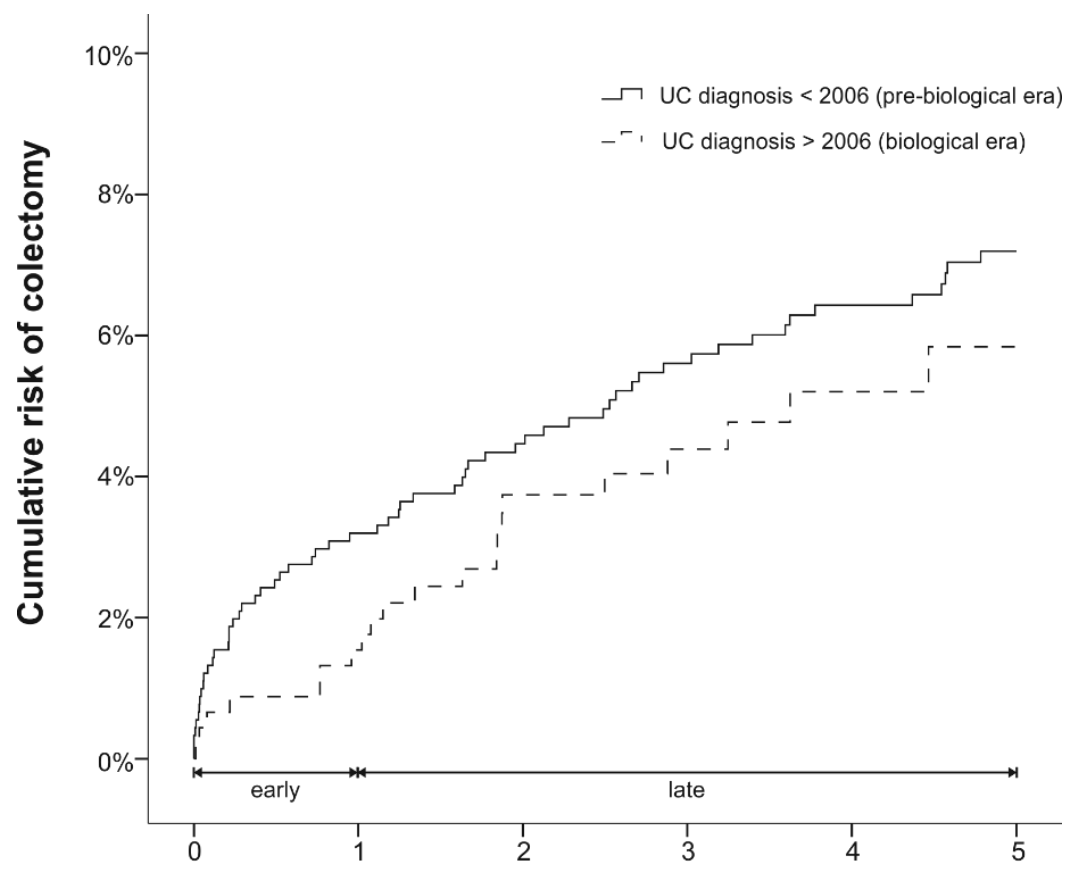

Disease duration in years

Figure 2.2. Kaplan-Meier curves of cumulative colectomy risk in UC patients diagnosed before and after 2006. Early colectomy rate was found to be 2.4-fold lower in UC patients diagnosed after 2006, the year biologicals were first registered for UC in the Netherlands, as compared to patients diagnosed in the era before biological availability $[<2006]$. In-depth focus showed these colectomies to be prevented rather than postponed. 


\section{IBDSL biobank}

IBDSL biobank studies focused on underlying disease mechanisms and the identification of biomarkers that predict disease course. Clostridium difficile ${ }^{58}$ and enteropathogenic viruses, ${ }^{59}$ for instance, were found not to be common triggers for IBD exacerbations. Microbial composition, on the other hand, changed when remissive IBDSL patients went into subsequent exacerbation, but these changes were rather patient-specific than general. ${ }^{70}$ Importantly, use of thiopurine medication also had a significant impact on the microbial composition and diversity, and should be considered when studying the intestinal microbiota in relation to disease course. $^{70}$

In a recent analysis, we investigated whether VOCs in exhaled air can accurately differentiate between active CD and remission. ${ }^{73}$ lleocolonoscopy is currently the gold standard to assess disease activity but, as it is invasive and expensive, alternatives are warranted. In the IBDSL cohort, a set of 10 discriminatory VOCs was found to correctly differentiate between active CD and remission [sensitivity $=0.81$, specificity $=0.80$, area under the curve $=0.80]^{73}$

\section{Main strengths and weaknesses?}

A major strength lies in the population-based character of the cohort, with a completeness of 93\%. Population-based cohorts comprise the full spectrum of IBD phenotypes, from mild to severe disease course, in contrast to referral cohorts which tend to over-represent the latter. This makes IBDSL ideal to study hypotheses that need a population-based design [such as incidence studies, studies on prevalence of disease manifestation, natural disease course and potential modulators thereof], and to confirm findings of other 'referral' cohorts. Although $93 \%$ is very high, we acknowledge that not all eligible patients were identified. Selection bias, however, is not expected in view of the factors previously discussed.

In addition to the high level of completeness, the cohort is also characterized by its high stability. South Limburg is bordered by Belgium and Germany, and even though it is allowed, cross-border health care remains limited, as well as migration. ${ }^{6}$ South Limburg IBD patients tend to stay within the region and, as only the IBDSL consortium centers [and general practitioners] are entrusted with IBD care, these patients can be followed up quite easily. This provides an ideal setting for population-based research.

Another strength is the long-term and very detailed clinical, demographic and pharmacological data being collected for each patient since diagnosis. As follow-up is done biennially, and as we are connected to the Dutch resident registration [and other databases in future], IBDSL data remains up to date. Also recall bias is minimal, because most data are collected prospectively. 
Finally, the extensive collection of biomaterial is a major strength, and makes IBDSL well equipped for investigating underlying molecular, genetic and microbiological mechanisms that initiate or sustain IBD. These biomaterials can also be used for the identification of [noninvasive] markers to predict disease course and therapy response, or to validate such markers if derived from other cohorts. IBDSL is currently building the first population-based IBD biobank. Although it is not population-based yet, it does include a representative sample of our cohort.

Limitations lie in the strict inclusion criteria. The IBDSL cohort does currently not contain any information on IBD patients diagnosed during childhood, nor any IBD- $U$ patients. Plans to include these patients are in progress. Second, due to its invasiveness and the large scale of the cohort, endoscopy could not always be used to determine disease activity at time of biomaterial donation [gold standard]. Disease activity was determined by clinical activity scores instead, but these scores are somewhat subjective and, especially for CD, correlation with actual mucosal inflammation is moderate. ${ }^{74,75}$ To overcome this, we validated a combination of clinical activity scores and inflammation markers [i.e. C-reactive protein, fecal calprotectin] in the IBDSL endoscopy subgroup, and found this combi-score to improve distinction of active from inactive disease. ${ }^{76}$

\section{Can I get hold of the data?}

We welcome new collaborations to study interesting hypotheses in IBD. Applications for collaboration are first to be approved by the IBDSL committee. Applications should be addressed to Dr Marie Pierik, principal investigator of the IBDSL cohort at IBDSL@maastrichtuniversity.nl. 


\section{References}

1. Romberg-Camps MJ, Bol Y, Dagnelie PC et al. Fatigue and health-related quality of life in inflammatory bowel disease: results from a population-based study in the Netherlands: the IBD-South Limburg cohort. Inflamm Bowel Dis 2010;16:2137-47.

2. Bernklev T, Jahnsen J, Lygren I, et al. Health-related quality of life in patients with inflammatory bowel disease measured with the short form-36: psychometric assessments and a comparison with general population norms. Inflamm Bowel Dis 2005;11:909-18.

3. Casellas F, Arenas JI, Baudet JS et al. Impairment of health-related quality of life in patients with inflammatory bowel disease: a Spanish multicenter study. Inflamm Bowel Dis 2005; 11:488-96.

4. Zhang YZ, Li YY. Inflammatory bowel disease: pathogenesis. World J Gastroentero/ 2014;20:91-99.

5. Stockbrugger RW, Russel MG, van Blankenstein M, Shivananda S. EC-IBD: a European effort in inflammatory bowel disease. Eur J Intern Med 2000;11:187-90.

6. StatisticsNetherlands. 2014. http://statline.cbs.nl/Statweb/

7. Lennard-Jones JE. Classification of inflammatory bowel disease. Scand J Gastroenterol Supp/ 1989;170:2-6; discussion 16-19.

8. Casparie M, Tiebosch AT, Burger $G$ et al. Pathology databanking and biobanking in The Netherlands, a central role for PALGA, the nationwide histopathology and cytopathology data network and archive. Cell Oncol 2007;29:19-24.

9. Satsangi J, Silverberg MS, Vermeire S, Colombel JF. The Montreal classification of inflammatory bowel disease: controversies, consensus, and implications. Gut 2006;55:749-53

10. Jeuring $S$, Van den Heuvel T, Zeegers M et al. OP005. Is elderly-onset ulcerative colitis a different entity? - Natural disease course and treatment response compared to adult-onset disease in the population-based IBD-SL cohort. J Crohns Colitis 2014;8: S3-S4.

11. Harvey RF, Bradshaw JM. A simple index of Crohn's-disease activity. Lancet 1980;1:514.

12. Walmsley RS, Ayres RC, Pounder RE, Allan RN. A simple clinical colitis activity index. Gut 1998;43:29-32.

13. Biobank [MUMC]. 2014. http://www.biobank.nl/

14. Daperno M, D'Haens G, Van Assche $G$ et al. Development and validation of a new, simplified endoscopic activity score for Crohn's disease: the SES-CD. Gastrointest Endosc 2004;60: 505-12.

15. Schroeder KW, Tremaine WJ, IIstrup DM. Coated oral 5-aminosalicylic acid therapy for mildly to moderately active ulcerative colitis. A randomized study. N Eng/ J Med 1987;317: 1625-29.

16. Sysmed-IBD. 2012. http://www.sysmedibd.eu/

17. IBD-BIOM. 2012. http://www.ibdbiom.eu/

18. IBD-Character. 2012. http://www.ibdcharacter.eu/

19. Romberg-Camps M, Kuiper E, Schouten $L$ et al. Mortality in inflammatory bowel disease in the Netherlands 1991-2002: results of a population-based study: the IBD South-Limburg cohort. Inflamm Bowel Dis 2010;16:1397-410.

20. Romberg-Camps MJ, Dagnelie PC, Kester AD et al. Influence of phenotype at diagnosis and of other potential prognostic factors on the course of 
inflammatory bowel disease. Am J Gastroentero/2009;104:371-83.

21. Romberg-Camps MJ, Hesselink-van de Kruijs MA, Schouten LJ et al. Inflammatory Bowel Disease in South Limburg [the Netherlands] 1991-2002: Incidence, diagnostic delay, and seasonal variations in onset of symptoms. $J$ Crohns Colitis 2009;3:115-24.

22. Russel MG, Dorant E, Brummer RJ et al. Appendectomy and the risk of developing ulcerative colitis or Crohn's disease: results of a large case-control study. South Limburg Inflammatory Bowel Disease Study Group. Gastroenterology 1997;113:377-82.

23. Russel MG, Dorant E, Volovics A et al. High incidence of inflammatory bowel disease in The Netherlands: results of a prospective study. The South Limburg IBD Study Group. Dis Colon Rectum 1998;41:33-40.

24. Russel MG, Engels LG, Muris JW et al. Modern life in the epidemiology of inflammatory bowel disease: a casecontrol study with special emphasis on nutritional factors. Eur J Gastroenterol Hepatol 1998;10:243-49.

25. Russel MG, Nieman FH, Bergers JM, Stockbrugger RW. Cigarette smoking and quality of life in patients with inflammatory bowel disease. South Limburg IBD Study Group. Eur J Gastroenterol Hepatol 1996;8:107581.

26. Russel MG, Pastoor CJ, Brandon S et al. Validation of the Dutch translation of the Inflammatory Bowel Disease Questionnaire [IBDQ]: a health-related quality of life questionnaire in inflammatory bowel disease. Digestion 1997:58:282-88.

27. Russel MG, Pastoor CJ, Janssen KM et al. Familial aggregation of inflammatory bowel disease: a population-based study in South Limburg, The Netherlands. The South Limburg IBD Study Group. Scand
J Gastroentero/ Supp/ 1997;223:8891.

28. Russel MG, Ryan BM, Dagnelie PC et al. Insurance problems among inflammatory bowel disease patients: results of a Dutch population based study. Gut 2003;52:358-62.

29. Russel MG, Stockbrugger RW. Is appendectomy a causative factor in ulcerative colitis? Eur J Gastroenterol Hepatol 1998;10: 455-57.

30. Russel MG, Volovics A, Schoon EJ et al. Inflammatory bowel disease: is there any relation between smoking status and disease presentation? European Collaborative IBD Study Group. Inflamm Bowel Dis 1998;4:182-86.

31. Wolters FL, Russel MG, Sijbrandij J et al. Phenotype at diagnosis predicts recurrence rates in Crohn's disease. Gut 2006;55:1124-30.

32. Wolters FL, Russel MG, Sijbrandij J et al. Crohn's disease: increased mortality 10 years after diagnosis in a Europe-wide population based cohort. Gut 2006;55:510-18.

33. Wolters FL, Russel MG, Sijbrandij J et al. Disease outcome of inflammatory bowel disease patients: general outline of a Europewide population-based 10-year clinical follow-up study. Scand J Gastroenterol Supp/2006;243:46-54.

34. Wolters FL, Russel MG, Stockbrugger RW. Systematic review: has disease outcome in Crohn's disease changed during the last four decades? Aliment Pharmacol Ther 2004;20:483-96.

35. Wolters FL, van Zeijl G, Sijbrandij J et al. Internet-based data inclusion in a population-based European collaborative follow-up study of inflammatory bowel disease patients: description of methods used and analysis of factors influencing response rates. World J Gastroenterol 2005;11:7152-58.

36. Boonen A, Dagnelie PC, Feleus A et al. The impact of inflammatory bowel 
disease on labor force participation: results of a population sampled casecontrol study. Inflamm Bowel Dis 2002;8:382-89.

37. Cohen RD. IBD indirect costs: the sleeping giant? Gastroenterology 2003;125:982-84.

38. van der Eijk I, Sixma H, Smeets Tet al. Quality of health care in inflammatory bowel disease: development of a reliable questionnaire [QUOTE-IBD] and first results. Am J Gastroenterol 2001;96:3329-36.

39. van der Eijk I, Stockbrugger R, Russel M. Influence of quality of care on quality of life in inflammatory bowel disease [IBD]: literature review and studies planned. Eur J Intern Med 2000;11:228-34.

40. Van Der Eijk I, Verheggen FW, Russel MG et al. "Best practice" in inflammatory bowel disease: an international survey and audit. Eur J Intern Med 2004; 15:113-20.

41. van der Eijk I, Vlachonikolis IG, Munkholm $P$ et al. The role of quality of care in health-related quality of life in patients with IBD. Inflamm Bowel Dis 2004; 10:392-98.

42. Fornaciari G, Salvarani C, Beltrami M, Macchioni P, Stockbrugger RW, Russel MG. Muscoloskeletal manifestations in inflammatory bowel disease. Can J Gastroenterol2001;15:399-403.

43. Geerling BJ, Badart-Smook A, Stockbrugger RW, Brummer RJ. Comprehensive nutritional status in patients with long-standing Crohn disease currently in remission. Am J Clin Nutr 1998;67:919-26.

44. Geerling BJ, Badart-Smook A, Stockbrugger RW, Brummer RJ. Comprehensive nutritional status in recently diagnosed patients with inflammatory bowel disease compared with population controls. Eur J Clin Nutr 2000;54:514-21
45. Geerling BJ, Badart-Smook A, van Deursen $\mathrm{C}$ et al. Nutritional supplementation with N-3 fatty acids and antioxidants in patients with Crohn's disease in remission: effects on antioxidant status and fatty acid profile. Inflamm Bowel Dis 2000;6:77-84.

46. Geerling BJ, Dagnelie PC, Badart-Smook A, Russel MG, Stockbrugger RW, Brummer RJ. Diet as a risk factor for the development of ulcerative colitis. Am J Gastroentero/2000;95: 1008-13.

47. Geerling BJ, Stockbrugger RW, Brummer RJ. Nutrition and inflammatory bowel disease: an update. Scand J Gastroenterol Supp/ 1999;230:95-105.

48. Gilissen LP, Bierau J, Derijks LJ et al. The pharmacokinetic effect of discontinuation of mesalazine on mercaptopurine metabolite levels in inflammatory bowel disease patients. Aliment Pharmacol Ther 2005;22:60511.

49. Gilissen LP, Derijks LJ, Driessen A et al. Toxicity of 6-thioguanine: no hepatotoxicity in a series of IBD patients treated with long-term, low dose 6thioguanine. Some evidence for dose or metabolite level dependent effects? Dig Liver Dis 2007;39:156-59.

50. Hoie O, Aamodt G, Vermeire S et al. Serological markers are associated with disease course in ulcerative colitis. A study in an unselected population-based cohort followed for 10 years. J Crohns Colitis 2008;2:114-22.

51. Hoie O, Schouten LJ, Wolters FL et al. Ulcerative colitis: no rise in mortality in a European-wide population based cohort 10 years after diagnosis. Gut 2007;56:497-503.

52. Hoie $\mathrm{O}$, Wolters F, Riis $L$ et al. Ulcerative colitis: patient characteristics may predict 10-yr disease recurrence in a European-wide population-based cohort. Am J Gastroentero/ 2007; 102:1692701. 
53. Isene $\mathrm{R}$, Bernklev $\mathrm{T}$, Hoie $\mathrm{O}$ et al. Thromboembolism in inflammatory bowel disease: results from a prospective, population-based European inception cohort. Scand J Gastroenterol 2014;49:820-25.

54. Juillerat $P$, Pittet $V$, Mottet $C$ et al. Appropriateness of early management of newly diagnosed Crohn's disease in a European population-based cohort. Scand J Gastroentero/ 2010;45:144956.

55. Katsanos KH, Tatsioni A, Pedersen N et al. Cancer in inflammatory bowel disease 15 years after diagnosis in a populationbased European Collaborative follow-up study. J Crohns Colitis 2011;5:430-42.

56. Katsanos KH, Vermeire S, Christodoulou DK et al. Dysplasia and cancer in inflammatory bowel disease 10 years after diagnosis: results of a populationbased European collaborative follow-up study. Digestion 2007;75:113-21.

57. Lennard-Jones JE, Shivananda S. Clinical uniformity of inflammatory bowel disease a presentation and during the first year of disease in the north and south of Europe. EC-IBD Study Group. Eur J Gastroenterol Hepatol 1997:9:353-59.

58. Masclee GM, Penders J, Jonkers DM, et al. Is clostridium difficile associated with relapse of inflammatory bowel disease? results from a retrospective and prospective cohort study in the Netherlands. Inflamm Bowel Dis 2013;19:2125-31.

59. Masclee GM, Penders J, Pierik M, et al. Enteropathogenic viruses: triggers for exacerbation in IBD? A prospective cohort study using real-time quantitative polymerase chain reaction. Inflamm Bowel Dis 2013;19:124-31.

60. Munkholm P. Pregnancy, fertility, and disease course in patients with Crohn's disease and ulcerative colitis. Eur J Intern Med 2000;11:215-21.
61. Reinders CA, Jonkers $D$, Janson EA et al. Rectal nitric oxide and fecal calprotectin in inflammatory bowel disease. Scand J Gastroentero/2007:42:1151-57.

62. Salvarani C, Vlachonikolis IG, van der Heijde DM et al. Musculoskeletal manifestations in a population-based cohort of inflammatory bowel disease patients. Scand J Gastroenterol 2001;36:1307-13.

63. Schoon EJ, Blok BM, Geerling BJ, et al. Bone mineral density in patients with recently diagnosed inflammatory bowel disease. Gastroenterology 2000; 119:1203-08.

64. Schoon EJ, Bollani S, Mills PR et al. Bone mineral density in relation to efficacy and side effects of budesonide and prednisolone in Crohn's disease. Clin Gastroenterol Hepatol 2005;3:113-21.

65. Schoon EJ, Geerling BG, Van Dooren IM et al. Abnormal bone turnover in longstanding Crohn's disease in remission. Aliment Pharmacol Ther 2001;15:78392.

66. Schoon EJ, Muller MC, Vermeer $\mathrm{C}$, et al. Low serum and bone vitamin $\mathrm{K}$ status in patients with longstanding Crohn's disease: another pathogenetic factor of osteoporosis in Crohn's disease? Gut 2001;48:473-77.

67. Schoon EJ, van Nunen AB, Wouters RS, et al. Osteopenia and osteoporosis in Crohn's disease: prevalence in a Dutch population-based cohort. Scand J Gastroenterol Supp/2000;232:43-47.

68. Shivananda S, Lennard-Jones J, Logan $\mathrm{R}$ et al. Incidence of inflammatory bowe disease across Europe: is there a difference between north and south? Results of the European Collaborative Study on Inflammatory Bowel Disease [EC-IBD]. Gut 1996;39:690-97.

69. Witte J, Shivananda S, Lennard-Jones JE et al. Disease outcome in inflammatory bowel disease: mortality, morbidity and therapeutic management 
of a 796-person inception cohort in the European Collaborative Study on Inflammatory Bowel Disease [EC-IBD]. Scand J Gastroentero/ 2000;35:127277.

70. Wills ES, Jonkers DM, Savelkoul PH et al.. Fecal microbial composition of ulcerative colitis and Crohn's disease patients in remission and subsequent exacerbation. PLoS One 2014;9:e90981.

71. Zankel E, Rogler G, Andus T, et al. Crohn's disease patient characteristics in a tertiary referral center: comparison with patients from a population-based cohort. Eur J Gastroenterol Hepatol 2005; 17:395-401.

72. Jeuring $S$, Bours $P$, Ambergen T et al. Effect of introduction of anti-tumour necrosis factor agents on the natural disease course of ulcerative colitis - a population-based IBD-SL study. Gastroenterology 144:S633.

73. Bodelier A, Pierik M, Dallinga J et al. Analysis of volatile organic compounds in exhaled air as a non invasive biomarker for inflammatory bowel disease [IBD]. Gastroenterology 2009;136:A35.

74. Bjorkesten CG, Nieminen U, Turunen U, et al. Surrogate markers and clinical indices, alone or combined, as indicators for endoscopic remission in antiTNFtreated luminal Crohn's disease. Scand J Gastroentero/2012;47:528-37.

75. Cellier C, Sahmoud T, Froguel E et al. Correlations between clinical activity, endoscopic severity, and biological parameters in colonic or ileocolonic Crohn's disease. A prospective multicenter study of 121 cases. The Groupe d'Etudes Therapeutiques des Affections Inflammatoires Digestives. Gut 1994;35:231-35.

76. Bodelier A, Jonkers D, van den Heuvel T et al. Diagnostic value of a novel combination score of disease activity in a real-life IBD cohort. ECCO congress. 2015. 



\section{CHAPTER 3}

A 20-Year Temporal Change Analysis in Incidence, Presenting Phenotype and Mortality, in the Dutch IBDSL Cohort Can Diagnostic Factors Explain the Increase in IBD Incidence?

Steven FG Jeuring*, Tim RA van den Heuvel*, Maurice P Zeegers, Dorien HE van Dongen, Anouk Wolters, Ad AM Masclee, Wim H Hameeteman, Marielle JL Romberg-Camps, Liekele E Oostenbrug, Marieke J Pierik, Daisy MAE Jonkers * both authors contributed equally to this work J Crohns Colitis. 2017 Oct 1;11(10):1169-1179 


\section{Abstract}

\section{Background}

The aim was to study temporal changes in incidence, disease phenotype at diagnosis, and mortality of adult inflammatory bowel disease [IBD] patients in South Limburg, The Netherlands, diagnosed between 1991 and 2010. In addition, the 2010 IBD prevalence was estimated.

\section{Methods}

A multi-faceted approach including hospital administrations, the national pathology registry [PALGA], and general practitioners led to the identification of 1162 patients with Crohn's disease [CD], 1663 with ulcerative colitis [UC], and 84 with unclassified IBD [IBD-U]. Temporal changes in incidence, disease phenotype, and mortality were studied using linear, multinomial regression analyses, and standardized mortality rates [SMR], respectively.

\section{Results}

The annual incidences increased from 17.90/100,000 in 1991 to 40.36/100,000 in 2010 for $\mathrm{IBD}$, from $5.84 / 100,000$ to $17.49 / 100,000$ for $C D$, and from $11.67 / 100,000$ to $21.47 / 100,000$ for UC [ $p<0.01$ for all]. A shift towards milder disease at diagnosis was observed over time [e.g. decrease of complicated disease in $C D$, increase of proctitis in UC]. IBD mortality was similar to that in the general population [SMR 0.92; 95\% confidence interval [CI] 0.81-1.05] and did not change over time. The estimated IBD prevalence was $830 / 100,000$.

\section{Conclusions}

The IBD incidence in South Limburg increased significantly between 1991 and 2010. The shift towards milder disease at diagnosis in parallel with the improved diagnostics and ability to detect low-grade inflammation was suggestive of an important role of diagnostic factors in this increase. Environmental factors probably played a role as well. The mortality was low and, together with the increasing incidence, led to the high prevalence of IBD in South Limburg. 


\section{Introduction}

Inflammatory bowel disease [IBD] is a chronic inflammatory condition of the intestine, encompassing the subtypes Crohn's disease [CD], ulcerative colitis [UC], and unclassified IBD [IBD-U]. IBD is characterized by sequences of exacerbation and remission, and is considered to arise from complex interactions between an altered intestinal immune response, the intestinal microbiota, and environmental exposures in a genetically susceptible host. ${ }^{1,2}$ This complex background contributes to the heterogeneous clinical presentation and disease course. ${ }^{3,4}$ IBD may have severe impact on a patient's [quality of] life, ${ }^{5}$ but also society endures trough high health care costs [e.g. due to loss of productivity or work absenteeism]. ${ }^{6}$ In 2013, the European prevalence was estimated at 0.3\% [equaling 2.5-3 million persons]. ${ }^{6}$

For local health planning, but also for allocation of funding money and patient communication, it is important to understand the current and future [societal] burden of IBD. Temporal change analyses in IBD epidemiology show that the prevalence is expected to further increase in future ${ }^{7}$, as IBD incidences are rising in virtually every region of the world ${ }^{7}$, and mortality in IBD is only marginally increased compared with the general population. ${ }^{8}$ Considering the widespread use of epidemiologic data, their validity is of utmost importance. Many data on [temporal changes in] IBD epidemiology, however, are derived from selected populations or administrative databases that are not designed for research purposes. Both may introduce methodological problems. The former can be characterized by an underrepresentation of mildly diseased cases $^{9}$, and the latter are sensitive for suboptimal case ascertainment [misclassifying prevalent cases as incident, or overclassifying non-IBD cases as IBD] and/or for changes in inclusion criteria over time. ${ }^{10,11}$ In addition, most studies lack detail on diagnostic factors or presenting disease phenotype over time, whereas such data can be useful for interpreting any observed temporal changes. A verification of temporal changes in epidemiological data in a stable, detailed, longstanding, population-based cohort of IBD patients is, therefore, warranted.

Here, we report on temporal changes in incidence, disease phenotype at diagnosis, and mortality, in adult IBD patients from the Dutch population-based IBD South Limburg [IBDSL] cohort, diagnosed between 1991 and 2010. In addition, we estimated the 2010 prevalence of adult IBD patients in South Limburg. 


\section{Methods}

\section{Source population}

South Limburg is a geographical region in the south of the Netherlands, the greater part enclosed by Belgium and Germany [Figure 3.1]. In 1991, South Limburg comprised 638,781 inhabitants, and the population size declined to 607,784 in $2010 .{ }^{12}$ The vast majority of inhabitants are Caucasian [exact numbers were not retrievable for ethical reasons] and 59\% live in urban areas [defined as $>1000$ inhabitants per $\mathrm{km}^{2}$ ]. $^{12}$ Hospital care in South Limburg is provided by three hospitals including one university hospital [Maastricht University Medical Center+] and two general hospitals [Zuyderland Medical Centers, locations Heerlen and Sittard-Geleen]. Cross-border health care is limited and migration rates are rather low ${ }^{12}$, favoring population-based research in this region.

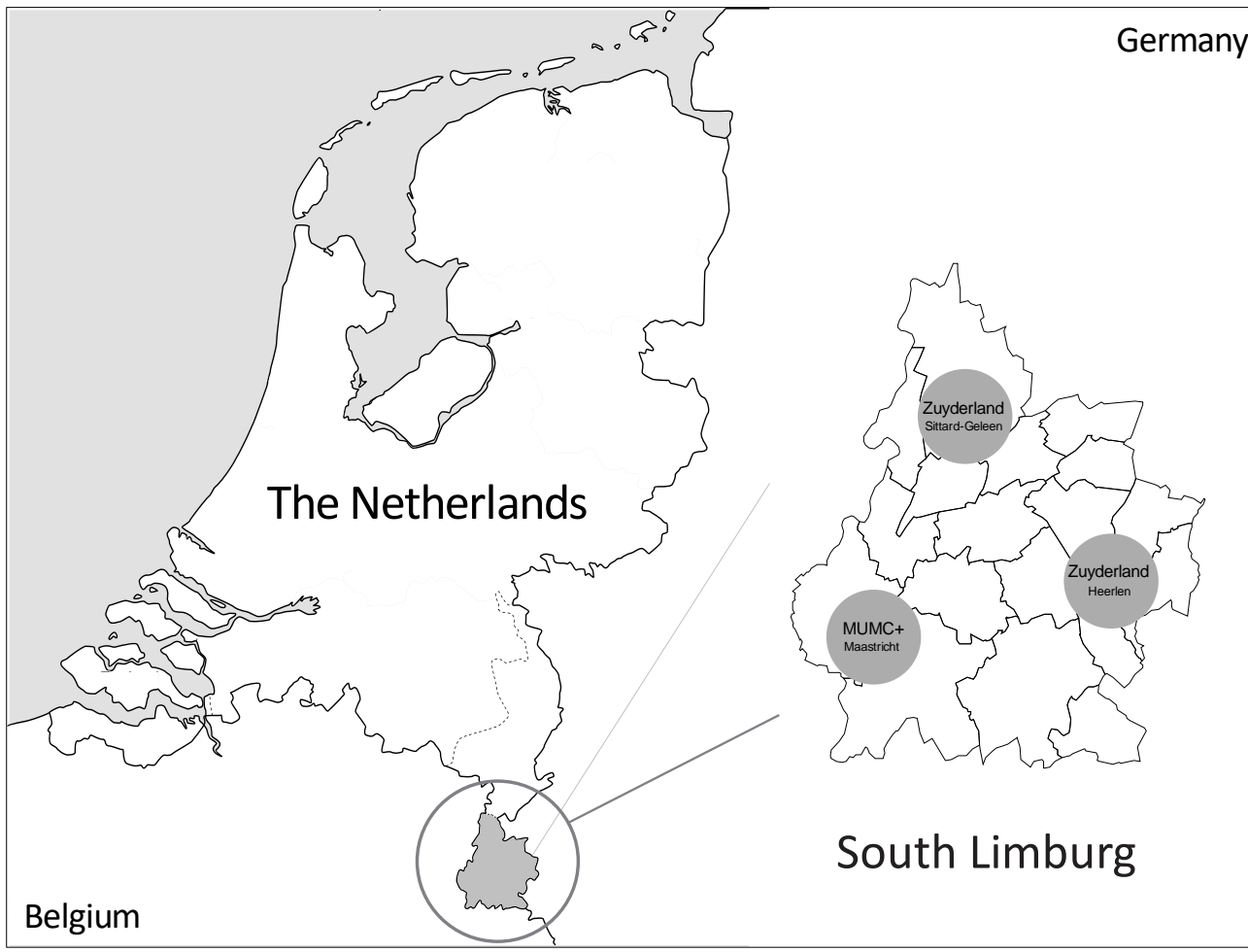

Figure 3.1. Geographical location of South Limburg, and the hospitals: Maastricht University Medical Center+ [MUMC+], Zuyderland Medical Center Heerlen, and Zuyderland Medical Center Sittard-Geleen. Adapted from van den Heuvel et al. ${ }^{13}$ 


\section{Study population}

All IBD patients from the population-based IBDSL cohort were eligible for the current study. For details on this cohort, we refer to the cohort profile. ${ }^{13}$ In brief, patients diagnosed with IBD between January 1, 1991 and December 31, 2010, while living in South Limburg and being over 18 years of age at diagnosis, were prospectively included and followed. A multi-faceted identification strategy, including the three regional hospitals, PALGA [the nationwide Dutch pathology database covering all pathology reports generated in the Netherlands since $1991]^{14}$ and general practitioners, resulted in over 93\% completeness. The remaining $7 \%$ was not likely to be associated with a specific IBD phenotype. ${ }^{13}$ IBDSL has been approved by the Ethics Committee of the Maastricht University Medical Center [NL31636.068.10], is registered in ClinicalTrial.gov [NCT02130349], and meets the ethical standards of the revised version of the Declaration of Helsinki. ${ }^{15}$

\section{Study endpoints and definitions}

Endpoints of the present study were temporal changes in incidence [1], disease phenotype at diagnosis [2], and mortality [3] of adult IBD patients between 1991 and 2010, and the IBD point prevalence in 2010 [4]. Endpoints were calculated for IBD [i.e. combination of CD, UC, and $\mathrm{IBD}-\mathrm{U}]$, and for $\mathrm{CD}$ and $\mathrm{UC}$ separately.

IBD was diagnosed according to the Lennard-Jones criteria ${ }^{16}$ and was confirmed by endoscopic, radiological, and/or histological findings. The date of endoscopic or radiological examination with first evidence of IBD was set as time of diagnosis. In case of a change in diagnosis over time, the latest diagnosis was used. For this reason, endpoints were not calculated separately for IBD-U, as most of these patients had a CD or UC diagnosis at a later stage.

Disease phenotype at diagnosis was characterized by age, disease location, and disease behavior, according to the Montreal consensus. ${ }^{17} \mathrm{CD}$ disease location groups were ileal [L1], colonic [L2], ileocolonic [L3], and isolated upper gastrointestinal [GI] disease [L4]. The presence of any upper $\mathrm{Gl}$ disease, either isolated or with concomitant lower $\mathrm{Gl}$ involvement, was also noted [ $L 4+\llcorner 4$ modifier]. $C D$ disease behavior groups were nonstricturing and non-penetrating [B1], stricturing [B2], or penetrating [B3]. The presence of perianal disease at diagnosis was also noted [P]. UC disease location groups were rectal disease [E1], left-sided disease [E2], and extensive disease [E3]. 


\section{Design and analyses}

Temporal changes in incidence The IBD incidence was expressed as the number of new IBD cases per 100,000 adult South Limburg inhabitants. All IBDSL cases were stratified by calendar year of diagnosis, age at diagnosis, and gender, and divided by calendar year-, age-, and gender-specific population rates from South Limburg. The latter were derived from Statistics Netherlands [CBS], responsible for official Dutch statistics. ${ }^{12}$ Incidence rates were calculated for every year between 1991 and 2010. Temporal changes in incidence were determined by linear regression analysis $[\alpha=0.05]$. Subsequently, Joinpoint regression analysis was conducted [post hoc], ${ }^{18}$ to identify multiple time trends within the study period. The average annual percentage changes [APC] were calculated for the total study period and for every observed Joinpoint trend. ${ }^{19}$ Differences in trends between males and females, and between $C D$ and $U C$, were tested for trend parallelism $[\alpha=0.05] . .^{20}$ To facilitate international comparisons, incidence rates were also standardized to the European standard population, using European Age Standardized Rates [EASR]. ${ }^{21}$

Temporal changes in disease phenotype at diagnosis Age was expressed as mean, and IBD location and IBD behavior as the proportion of patients per location or behavior group, respectively. The mean age at IBD diagnosis was calculated for every year between 1991 and 2010, and temporal changes were determined by linear regression analysis $[\alpha=0.05]$. The proportions of patients per location or behavior group were calculated for every year between 1991 and 2010, and temporal changes in distribution of the groups were determined by multinomial logistic regression analyses [ $\alpha=0.05]$. In case the distribution changed over time, a post hoc binary logistic regression analysis $[\alpha=0.05]$ was performed to study in which group[s] the proportion changed.

Temporal changes in mortality For the mortality analyses, patients were followed until December 31, 2012, or until lost to follow-up [i.e. death or permanent migration outside the region]. The IBD mortality was expressed as standardized mortality ratio [SMR], which is the ratio between observed $[\mathrm{O}]$ and expected $[\mathrm{E}]$ deaths. Data on observed deaths and migration were obtained through linkage to the national resident registration ${ }^{22}$, which is the official government database continuously updated on the vital status and addresses of all Dutch inhabitants. Expected deaths for the South Limburg region were based on data of Statistics Netherlands [CBS]. ${ }^{12}$ Calendar year-, age-, and gender-specific mortality rates were used to calculate the expected number of deaths for every patient year at risk, which were then accumulated into the overall expected number of deaths. Patient-years were recorded from the year of IBD diagnosis up to and including the year follow-up ends [i.e. loss to follow-up or 
end of study]. Patient-years in which the patient was not at risk were censored. Incomplete patient-years [i.e. years in which diagnosis, loss to follow-up or censoring occurred] were corrected for by considering them as having contributed half-person-years, in order to prevent overestimation. SMRs were calculated for the total cohort, and in order to study temporal changes, SMRs were also determined in two subcohorts based on the era of diagnosis [i.e. one subcohort of patients diagnosed between 1991 and 2000, and one subcohort of patients diagnosed between 2001 and 2010; follow-up ended 2 years after each era]. Confidence intervals were determined by Byar's approximation $[\alpha=0.05]$. Subanalyses were performed in each cohort for gender, location at diagnosis, and behavior at diagnosis. Only IBD patients aged $\geq 20$ years at diagnosis were included, as mortality rates of younger persons were not readily available.

Point prevalence The IBD prevalence was expressed as the number of prevalent IBD cases per 100,000 adult South Limburg inhabitants. Prevalence was determined by using cases from the IBDSL population [alive in 2010], completed with an estimation of alive prevalent South Limburg adult cases who did not fulfil the IBDSL inclusion criteria [e.g. IBD diagnosis before 1991 and patients diagnosed elsewhere but migrated into South Limburg]. The latter was done by a stepwise approach. First, current and historic [since 1991] registries of the participating IBDSL hospitals were used to identify every IBD patient not fulfilling IBDSL inclusion criteria. Second, corrections were applied for: [a] patients not having IBD; [b] deceased patients; and [c] patients migrated inside and outside the region. Correction factors were derived from a case-ascertainment check reviewing the medical files of 2000 random IBD patients from the participating hospitals. Then, the total number of prevalent IBD patients in 2010 was divided by the South Limburg adult population for that year. Additionally, the South Limburg prevalence was extrapolated in order to estimate IBD prevalence in the Netherlands [nationwide]. Therefore, the ratio of newly diagnosed IBD patients living in South Limburg to all newly diagnosed patients in the Netherlands was used. This ratio was determined by reviewing postal codes from a random sample of 5000 newly diagnosed IBD patients [diagnosed between 1991 and 2011], derived from PALGA. ${ }^{14}$ 


\section{Results}

In total, 2909 IBD patients [1162 CD, 1663 UC, and 84 IBD-U] were diagnosed between January 1, 1991 and December 31, 2010, and were included in the IBDSL cohort. Baseline characteristics are presented in Table 3.1.

\begin{tabular}{|c|c|c|c|c|c|}
\hline & & $\begin{array}{c}\text { IBD } \\
{[\mathrm{N}=2909]}\end{array}$ & $\begin{array}{c}C D \\
{[N=1162]}\end{array}$ & $\begin{array}{c}U C \\
{[N=1663]}\end{array}$ & $\begin{array}{c}\text { IBD-U } \\
{[N=84]}\end{array}$ \\
\hline Age in years & mean [SD] & $42.6[16.9]$ & $37.7[15.9]$ & $45.8[16.7]$ & $48.4[17.1]$ \\
\hline Male & $N[\%]$ & $1360[46.8]$ & $434[37.3]$ & $886[53.3]$ & $40[47.6]$ \\
\hline \multicolumn{6}{|l|}{ Location } \\
\hline L1, ileum & $N[\%]$ & - & $500[43.0]$ & - & - \\
\hline L2, colon & $N[\%]$ & - & $371[31.9]$ & - & - \\
\hline L3, ileocolon & $N[\%]$ & & $267[23.0]$ & - & - \\
\hline$\llcorner 4$, upper Gl & $N[\%]$ & - & $24[2.1]$ & - & - \\
\hline$\llcorner 4+\llcorner 4$ modifier & $N[\%]$ & & $124[10.7]$ & - & - \\
\hline E1, proctitis & $N[\%]$ & - & - & $565[34.2]$ & - \\
\hline E2, left-sided & $N[\%]$ & - & - & $789[47.8]$ & - \\
\hline E3, extensive & $N[\%]$ & - & - & $296[17.9]$ & - \\
\hline \multicolumn{6}{|l|}{ Behaviora $^{a}$} \\
\hline B1, inflammatory & $N[\%]$ & - & $900[77.5]$ & - & - \\
\hline $\mathrm{B} 2$, stricturing & $N[\%]$ & - & $177[15.2]$ & - & - \\
\hline B3, penetrating & $N[\%]$ & - & $85[7.3]$ & - & - \\
\hline P, perianal disease & $N[\%]$ & - & $94[8.1]$ & - & - \\
\hline
\end{tabular}

$\mathrm{IBD}$, inflammatory bowel disease; CD, Crohn's disease; UC, ulcerative colitis; IBD-U, IBD-undefined; N, number of patients; SD, standard deviation.

a phenotype according to Montreal Classification

${ }^{b}$ disease location for UC could not be retrieved in 11 cases

\section{Incidence}

In the total IBD group, the mean incidence rate was 27.49 per 100,000. Between 1991 and 2010, the IBD incidence increased significantly from 17.90 to 40.36 per 100,000, corresponding to an average APC of 4.35\% [95\% Cl 2.70-6.01] [Table 3.2, Figure 3.2]. A two-trend Joinpoint regression model showed the best fit. Between 1991 and 1999, the IBD incidence was found to be rather stable with an average APC of 0.39\% [95\% Cl-2.95-3.84]. Since 1999, an increase has been observed with an average APC of 7.32\% [95\% Cl 5.539.15]. 


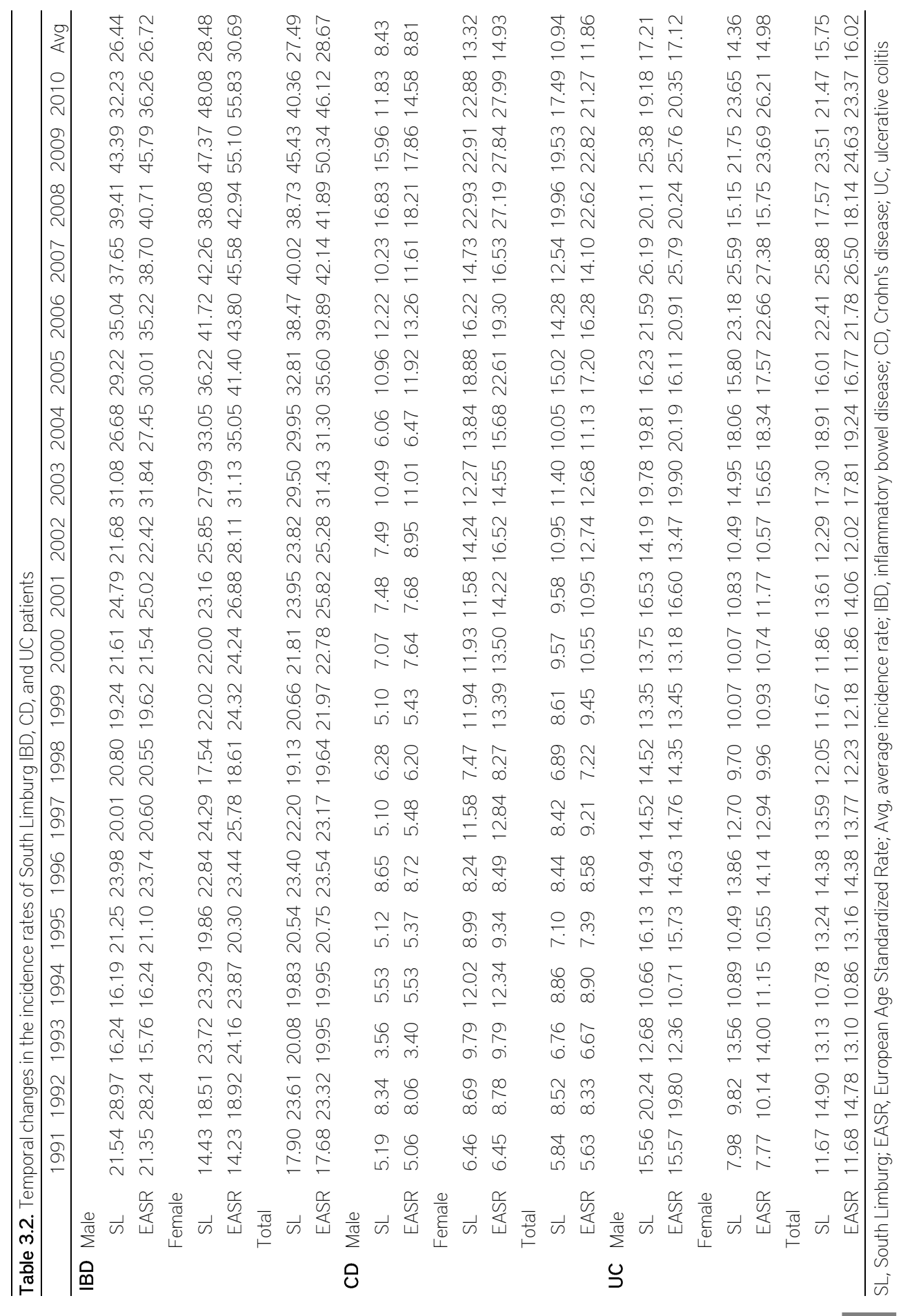



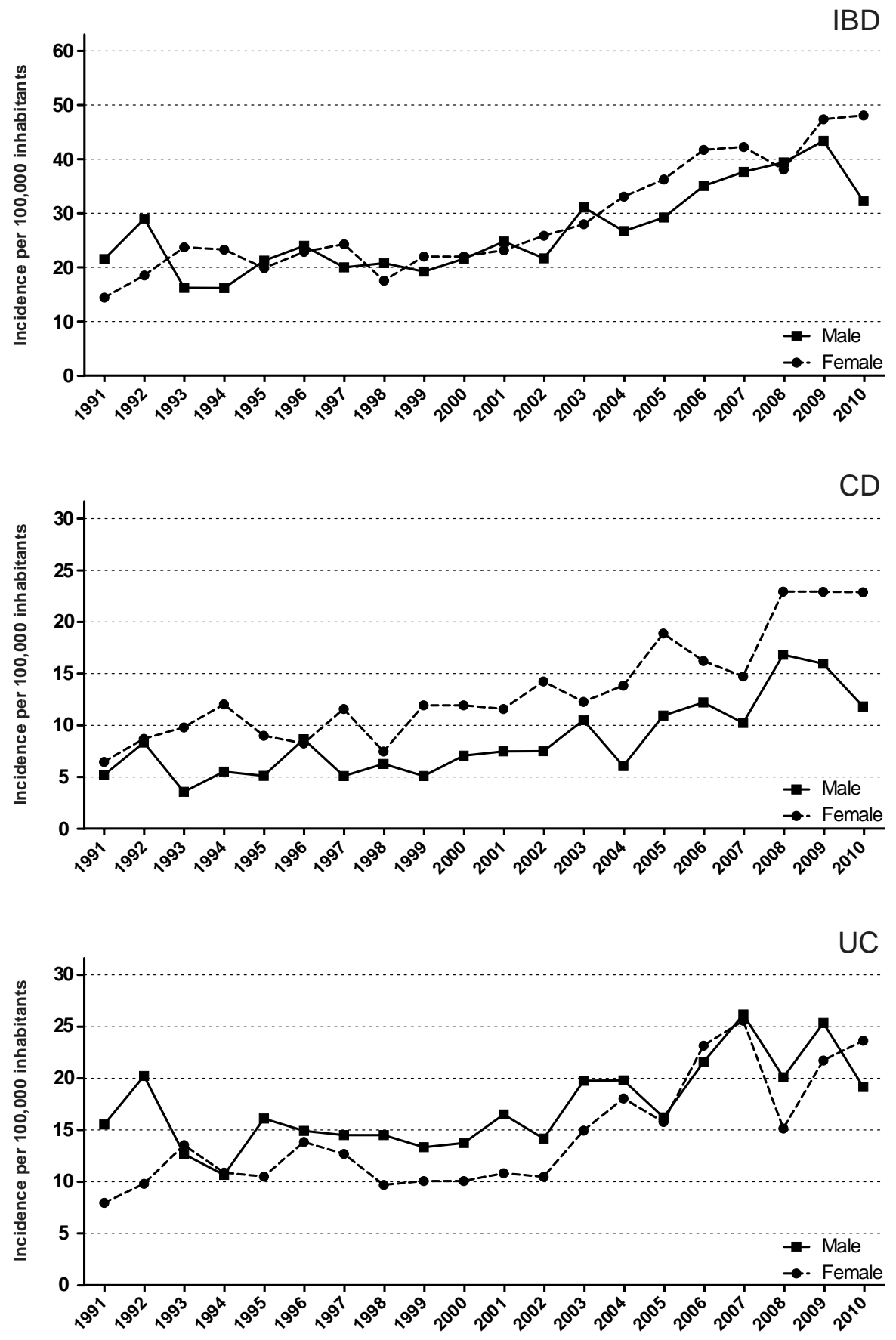

Figure 3.2. Temporal changes in incidence rates of South Limburg IBD, CD, and UC patients. IBD, inflammatory bowel disease; CD, Crohn's disease; UC, ulcerative colitis. 
For CD, the mean incidence rate was 10.94 per 100,000. Between 1991 and 2010, the CD incidence increased significantly from 5.84 to 17.49 per 100,000, corresponding to an average APC of $5.97 \%$ [95\% Cl 4.68-7.28] [Table 3.2, Figure 3.2]. No differences in temporal changes were observed between genders [males: average APC $5.87 \%, 95 \% \mathrm{Cl}$ 3.73-8.06, females: average APC 5.88\%, 95\% Cl 4.51-7.26, $p=0.99$ ]. Also for CD, a twotrend Joinpoint regression model showed the best fit. Between 1991 and 1998, the CD incidence was rather stable [average APC 1.12\%, 95\% Cl -5.21-7.88], followed by an increase [average APC: $7.79 \%, 95 \%$ Cl 5.44-10.19].

For UC, the mean incidence rate was 15.75 per 100,000. Between 1991 and 2010, the UC incidence increased significantly from 11.67 to 21.47 per 100,000 , corresponding to an average APC of 3.79\% [95\% Cl 2.39-5.21] [Table 3.2, Figure 3.2]. No differences in temporal changes were observed between genders [males: average APC $2.60 \%, 95 \% \mathrm{Cl}$ 1.11-4.11, females: average APC 5.07\%,95\% Cl 3.27-6.90, $p=0.06$ ]. Also for UC, a twotrend Joinpoint regression model showed the best fit. The UC incidence was stable between 1991 and 1999 [average APC $-0.75 \%, 95 \% \mathrm{Cl}-6.04-4.83$ ], followed by an increase [average APC 6.42\%, 95\% Cl 3.30-9.65].

Overall, the incidence of $\mathrm{CD}$ showed a stronger increase over time than the incidence of $U C[p=0.04]$, resulting in a decrease in UC:CD ratio from 1.76 in patients diagnosed between 1991 and 1995 to 1.39 in those diagnosed between 2006 and 2010 [average APC $-2.01 \%, 95 \% \mathrm{Cl}-3.68$ to -0.30$]$.

\section{Disease phenotype at diagnosis}

For $\mathrm{CD}$, the mean age at diagnosis increased from 35.0 years [standard deviation [SD] 14.8] to 36.9 years [SD 15.5], between 1991 and 2010 [ $p<0.01$ ]. L1 was the most common location at diagnosis [43.0\%] [Table 3.1]. Over time, a shift in the distribution of disease location was observed in the multinomial logistic regression analysis [ $p=0.03]$, and post hoc analyses showed a decrease in $\mathrm{L} 1$ [average APC $-1.87 \%, 95 \% \mathrm{Cl}-2.75$ to -0.98 ] [Figure 3.3]. Upper $G$ l disease, either isolated or with concomitant lower Gl involvement [ $L 4+L 4$ modifier], was diagnosed in $10.7 \%$ of the total CD population, and increased over time [average APC $4.01 \%, 95 \% \mathrm{Cl} 0.64-7.49]$. B1 disease was the most common behavior at diagnosis [77.5\%] [Table 3.1]. Over time, a shift in the distribution of disease behavior was observed in the multinomial logistic regression analysis $[p=0.01]$, and post hoc analyses showed a decrease in complicated disease [B2 + B3 combined, average APC -2.41, 95\% Cl -4.56 to -0.22], and a decrease in $\mathrm{B} 3$ alone [average APC $-5.44 \%, 95 \% \mathrm{Cl}-8.71$ to -2.06] [Figure 3.3]. Perianal disease $[\mathrm{P}$ ] was present in $8.1 \%$ and did not change over time [average APC $-1.55 \%, 95 \% \mathrm{Cl}$ $-3.81-0.77]$. 
DISEASE LOCATION CD

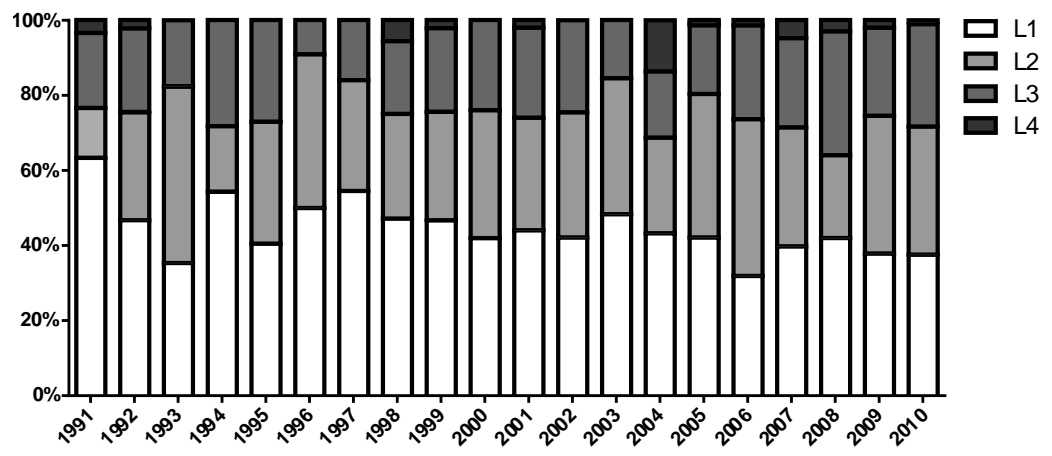

DISEASE BEHAVIOUR CD

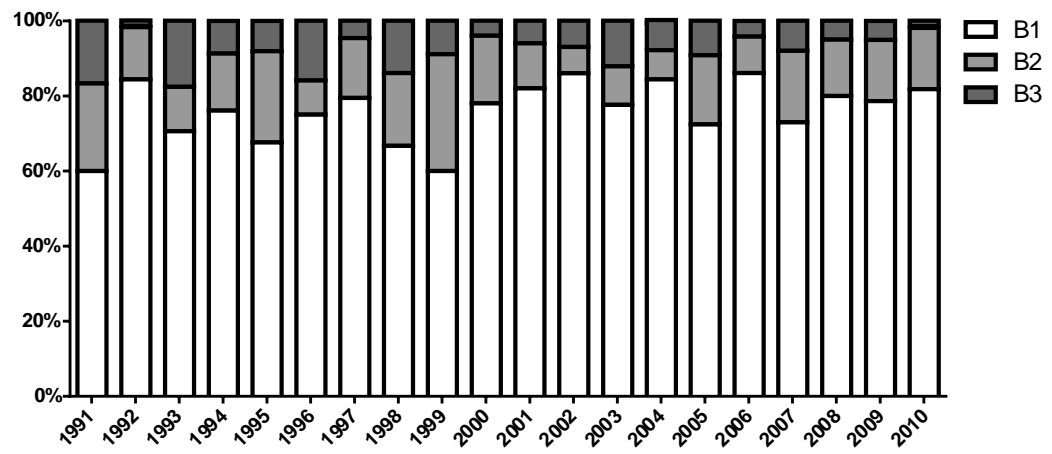

DISEASE LOCATION UC

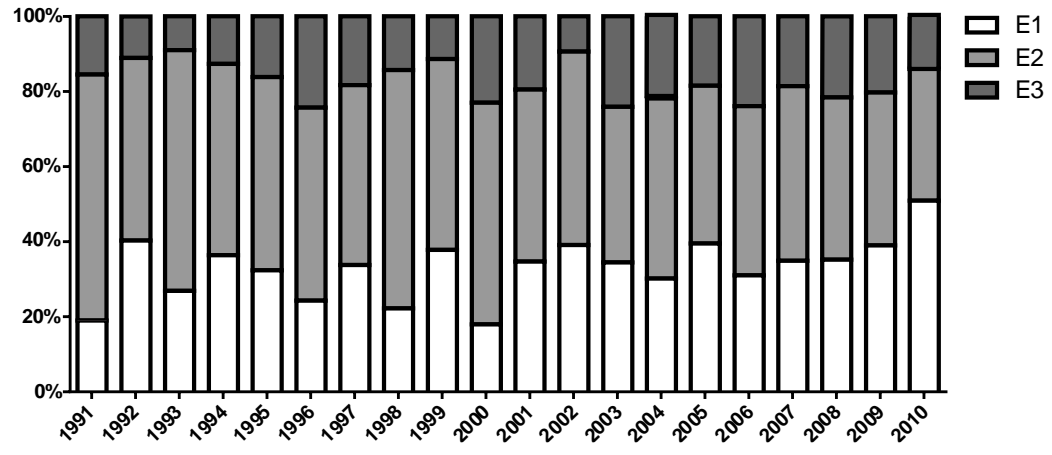

Figure 3.3. Temporal changes in phenotype distribution at diagnosis of South Limburg CD and UC patients. CD, Crohn's disease; UC, ulcerative colitis; phenotype was set at diagnosis, according to Montreal Classification. Disease location of CD was defined as ileal disease [L1], colonic disease [L2], ileocolonic disease [L3] or isolated upper gastrointestinal disease [L4]. Disease behavior of CD was defined as non-stricturing non-penetrating [B1], stricturing [B2] or penetrating [B3]. Disease location of UC was defined as ulcerative proctitis [E1], left sided UC [E2] and extensive UC [E3]. 
For UC, the mean age at diagnosis increased from 42.5 years [SD 17.0] to 45.9 years [SD 16.6] between 1991 and 2010 [ $p<0.01]$. E2 disease was the most common location at diagnosis [47.8\%] [Table 3.1]. Over time, a shift in the distribution of disease location was observed in the multinomial logistic regression analysis $[p<0.01]$, with the post hoc analyses showing an increase in E1 [average APC 1.87, 95\% Cl 0.28-3.48] and a decrease in E2 [average APC -2.14\%, 95\% Cl-3.00 to -1.28] [Figure 3.3].

\section{Mortality}

Of the 2909 IBD patients, 2804 IBD patients were included in the mortality analysis [27,405 patient-years] and $105 \mathrm{IBD}$ patients were excluded for previously mentioned reasons. Of the included patients, 1117 patients had CD [10,599.5 patient-years], 1603 had UC [16,139 patient-years], and 83 had IBD-U [666.5 patient-years].

For the total IBD group, mortality did not differ from that in the background population, either in the total cohort [SMR 0.92, 95\% Cl 0.81-1.05] in the 1991-2000 subcohort [SMR 0.89, 95\% Cl 0.66-1.16], or in the $2001-10$ subcohort [SMR $0.81,95 \% \mathrm{Cl}$ 0.64-1.01] [Table 3.3]. Results were similar in the gender-specific analyses. 


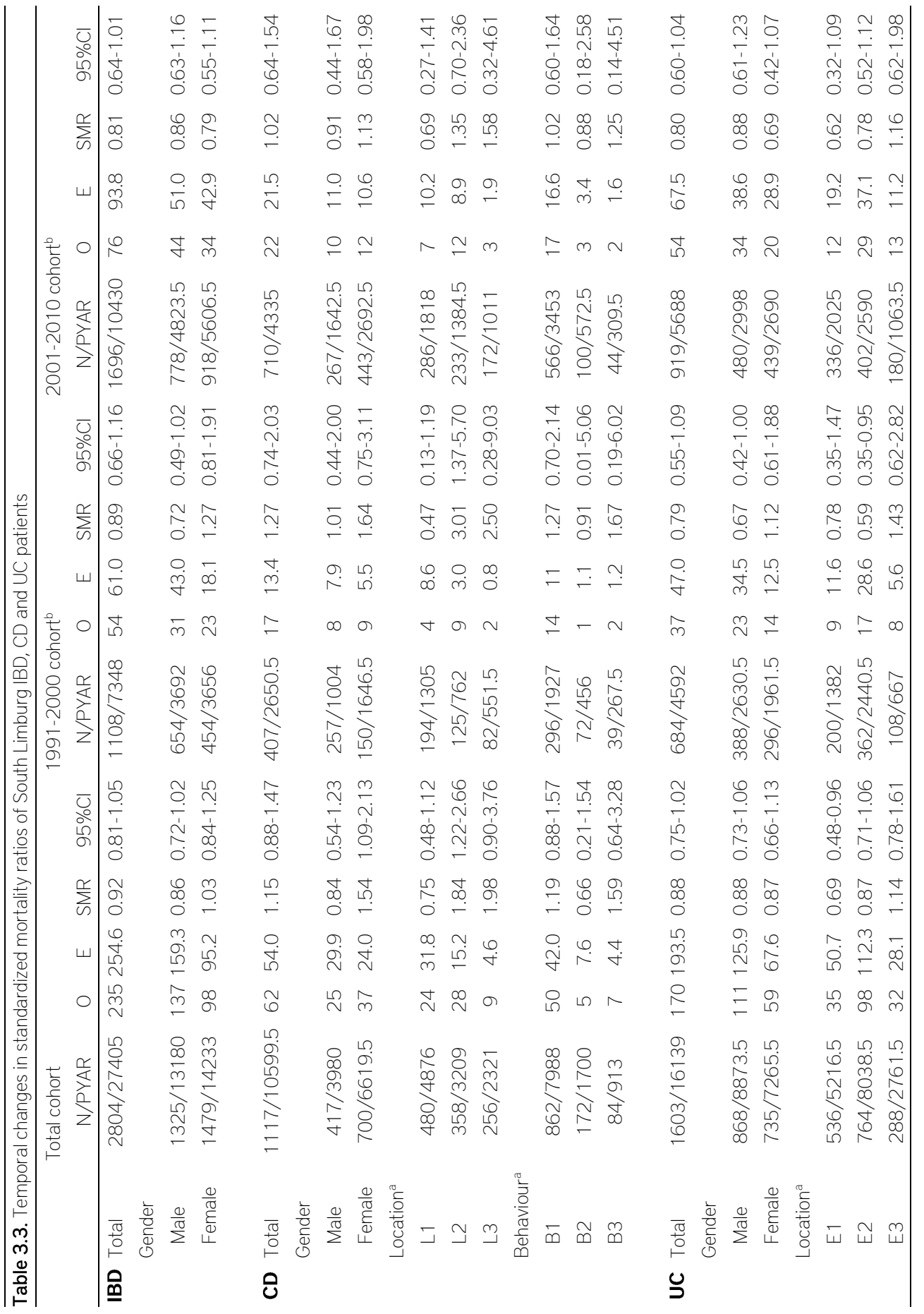


Table 3.3. Temporal changes in standardized mortality ratios of South Limburg IBD, CD and UC patients IBD, inflammatory bowel disease; CD, Crohn's disease; UC, ulcerative colitis; N, number of patients; PYAR, pers 1 onyears at risk; O, observed deaths; $\mathrm{E}$, expected deaths; SMR, standardized mortality ratio; 95\% Cl, 95\% confidence interval by Byar's approximation.

a phenotype at diagnosis, according to Montreal Classification. Disease location of CD was defined as ileal disease [L1], colonic disease [L2] or ileocolonic disease [L3]. Disease behavior of CD was defined as non-stricturing nonpenetrating [B1], stricturing [B2] or penetrating [B3]. Disease location of UC was defined as ulcerative proctitis [E1], left-sided UC [E2] and extensive UC [E3]

${ }^{b}$ follow-up ends 2 years after this period.

For CD and UC separately, mortality did not differ from that in the background population, either in the total cohort or in the era-specific subcohorts [Table 3.3]. The gender-specific analyses revealed an increased mortality risk for female CD patients in the total cohort [SMR 1.54, 95\% Cl 1.09-2.13]. In the phenotype-specific analyses, an increased mortality risk was observed for CD patients with colonic disease at diagnosis [L2] in the total CD cohort [SMR 1.84, 95\% Cl 1.22-2.66] and in the 1991-2000 CD subcohort [SMR 3.01, 95\% Cl 1.375.70] [Table 3.3]. In contrast, a decreased mortality risk was observed for UC patients with proctitis at diagnosis [E1] in the total cohort [SMR 0.69, 95\% Cl 0.48-0.96], and for leftsided UC patients in the 1991-2000 subcohort [SMR 0.59, 95\% Cl 0.35-0.95] [Table 3.3].

\section{Prevalence}

In 2010, the IBD prevalence in South Limburg was estimated at 830 per 100,000 for IBD [4186 prevalent cases in South Limburg], at 331 per 100,000 for CD [1670 cases], at 475 per 100,000 for UC [2395 prevalent cases], and at 24 per 100,000 for IBD-U [121 cases]. According to the national pathology registry PALGA, 5.2\% of newly diagnosed Dutch IBD cases lived in South Limburg. The nationwide Dutch IBD prevalence was therefore estimated to be 613 per 100,000 [approximately 80,627 prevalent cases]. 


\section{Discussion}

In this study, we reported on temporal changes in incidence, disease phenotype at diagnosis, and mortality in adult IBD patients from South Limburg, diagnosed between 1991 and 2010. The following key findings were observed: the incidences of IBD, and its subtypes CD and UC, have increased significantly over time. The proportion of CD patients with ileal disease at diagnosis [L1] and those with complicated disease at diagnosis [B2 + B3] decreased over time, whereas the proportion of UC patients with ulcerative proctitis at diagnosis [E1] was found to increase. IBD mortality rates were similar to background population mortality, but an increased risk was observed for female CD patients and CD patients with colonic disease at diagnosis [L2]. A decreased mortality risk was found for UC patients with ulcerative proctitis at diagnosis [E1]. Finally, the 2010 IBD prevalence was estimated at 830 per 100,000 for South Limburg and at 613 per 100,000 for The Netherlands [nationwide].

The observed IBD incidence rates in the region of South Limburg are among the highest in literature, and are in line with Molodecky et al.'s landmark review that showed higher incidences in Western countries when compared with the rest of the world. ${ }^{7}$ The high IBD incidence in South Limburg may be explained by several factors that have previously been associated with IBD, for instance easy health care access, the high levels of diagnostics and administration, a Western lifestyle, and the relatively high grade of urbanisation. ${ }^{12,23-26}$ In addition, South Limburg and neighboring regions in Belgium and Germany had intensive mining and coal industries in the 20th century, and still are rather heavily industrialized. Subsequent air pollution may have contributed to the high IBD incidence. 25,27,28

The South Limburg IBD incidence has increased significantly between 1991 and 2010, which is in keeping with epidemiological studies from other West European countries [Denmark, ${ }^{29}$ Sweden, ${ }^{30,31}$ and France ${ }^{32}$. In South Limburg, methodological factors are unlikely to play a role in this increase because identification methods have been stable throughout the entire study period. Also genetic influences are limited due to the restricted study length. The incidence increase can, therefore, best be explained by diagnostic and environmental factors, as elucidated below.

Several important diagnostic changes have been introduced during the study period, which most probably contribute to a part of the increase in IBD incidence. First, imaging techniques have advanced over the years; the quality of the endoscopes has improved ${ }^{33}$ and sensitive imaging techniques have been introduced in the participating hospitals, such as computed tomography [CT, introduced in 1994], video capsule endoscopy [VCE, in 2002] and magnetic resonance imaging [MRI, in 2006]. Second, the mode of diagnosis has shifted over the years. In CD, for instance, the most frequently used diagnostic tool between 1991 and 1995 was small bowel follow-through [in 56\% of all CD diagnoses, SBFT], followed by 
endoscopic procedures [49\%]. This gradually shifted towards 6\% and 89\% between 2006 and 2010 , respectively. In the latter period, CT was used in $15 \%$ of CD diagnoses, MRI in $13 \%$, and VCE in 5\%. Third, referral routines of general practitioners [GP] to secondary/tertiary care have been optimized in the catchment area, including direct referrals for endoscopic procedures by GPs. Finally, awareness of the disease has probably increased among patients, GPs, and specialists [e.g. easier access to information, regular IBD training for GPS, and the transition of gastroenterology care from specialists in internal medicine to trained gastroenterologists]. Altogether, these diagnostic changes point towards an under-diagnosis of mild IBD cases in the early years of the cohort as well as a relative late detection of IBD at that time, both contributing to the current increase in diagnosed IBD incidence. This hypothesis was supported by several observations in the IBDSL cohort. The proportion of CD patients with complicated disease at diagnosis [B2 + B3], for instance, decreased over time, and the proportion of UC patients with ulcerative proctitis [E1] increased. In addition, upper GI disease at diagnosis [ $L 4+\llcorner 4$ modifier] increased over time as well, which suggests that at least some of such cases were missed in the period preceding the diagnostic changes. Finally, previous IBDSL studies on the same study period have shown a significant attenuation of surgery at diagnosis, both in $C D$ and in $U C .{ }^{34,35}$ To what extent the diagnostic changes contributed to the increased IBD incidence remains speculative.

Despite the fact that only circumstantial evidence is available, part of the increase in IBD incidence in South Limburg is most probably attributable to environmental factors. The rapid rise in IBD incidence worldwide, ${ }^{7}$ the low concordance levels in IBD twin studies, ${ }^{36-39}$ the increasing IBD risk in immigrants migrating from low- to high-prevalence areas, ${ }^{40-42}$ and the spatial differences in IBD incidence within regions, ${ }^{7,43}$ have indicated the important role of environmental factors in the etiology of IBD. In addition, genome-wide association studies [GWAS] have found that genetic susceptibility is only responsible for approximately $30 \%$ of the IBD incidence, and the remaining contribution is mainly driven by the environment. ${ }^{7,44,45}$ Several environmental risk factors have already been associated with IBD [e.g. smoking, diet, childhood hygiene, socioeconomic factors, and many others $].{ }^{46}$ Altogether, this gives reason to believe that the observed increase in South Limburg IBD incidence is also partly driven by environmental factors, but elaborating on specific local factors remains speculation. The most profound environmental changes in South Limburg over the past decades are the attenuating socioeconomic status and increasing urbanization, both associated with IBD. ${ }^{26,43,47}$ Further research on IBD-related environmental exposures in South Limburg is important, but is beyond the scope of this study.

Several temporal changes in phenotype characteristics at diagnosis have been observed. First, the proportion of CD patients with ileal disease at diagnosis [L1] has decreased over time. In the early 90s, sigmoidoscopies and SBFT were commonly performed 
in the diagnostic workup for abdominal complaints in South Limburg, whereas colonoscopies, $\mathrm{CT}$, and MRI are currently preferred. It may be possible that proximal colonic involvement was occasionally missed in the early years, resulting in an $L 1$ rather than an $L 3$ classification. This temporal change must be taken into account when interpreting studies that use the Montreal classification. Second, the mean age at diagnosis for both CD and UC patients has increased over time. Increases, however, were subtle [1.9 years in CD and 3.4 years in UC between 1991 and 2010] and may very well be an effect of population ageing. ${ }^{12,48}$

The overall mortality rates in the South Limburg CD and UC populations were similar to the background population mortality and have not changed over time. In a recent metaanalysis by Bewtra et al., the mortality risk in inception cohorts was increased in CD [SMR $1.34,95 \% \mathrm{Cl} 1.15-1.56$ ] but not in UC [SMR 1.08, 95\% Cl 0.97-1.21]. ${ }^{8}$ Our results seem beneficial, but we must take into consideration that inception cohorts, and thus the IBDSL cohort, have a somewhat short duration to adequately study IBD-related death. Furthermore, the risk profile of some specific patient groups did differ from the background population. Female CD patients, for instance, had an increased risk for death. Of the 37 observed deaths, four were CD-related [stenosis-based ileus, euthanasia, complications after IBD surgery, and palliative care in a case of penetrating disease], six were possibly CD-related [colorectal carcinoma in five cases, and pneumonia within one month after IBD surgery], 10 were not CD-related, and 17 causes of death could not be retrieved from the patient files [and therefore a relation with $C D$ is not likely]. The reason for this increase is unclear, although we cannot exclude that it [in part] may be affected by the high percentage of female smokers at diagnosis $\left[53 \%\right.$ vs $44 \%$ in men]. ${ }^{49} \mathrm{CD}$ patients with colonic disease at diagnosis [L2] were also at increased risk for death. Of the 28 observed deaths, four were CD-related [euthanasia, complications after IBD surgery in two patients, and palliative care in a case of penetrating disease], three were possibly CD-related [colorectal carcinoma, cholangiocarcinoma, and pneumonia within 1 month after IBD surgery], eight were not CD-related, and 13 causes of death could not be retrieved. UC patients with ulcerative proctitis at diagnosis [E1] were at decreased risk for death.

The increasing incidence and low mortality, together with the chronic nature of IBD, and the often young age of onset, ${ }^{1,2}$ have contributed to the high IBD prevalence estimate in South Limburg [830 per 100,000]. The IBDSL cohort does not include pediatric IBD patients, and true prevalence rates may therefore even be [slightly] higher. The estimated nationwide IBD prevalence in the Netherlands is high as well [613 per 100,000, equivalent of 80,627 prevalent IBD cases in 2010], but lower when compared with South Limburg. This is in line with the overall lower health status in the South Limburg region [e.g. relative high prevalences of other chronic diseases, cancer, and depression, and a lower life expectation]. ${ }^{50,51}$ As preventive measures are not available yet, the observed findings give reason to believe that 
IBD prevalence will even further increase in future, and subsequently the burden for health care and society is expected to increase as well. The need for preventive measures is thus high, and their modifiable character make environmental factors an important target for interventions. More extensive research on [specific] environmental exposure is therefore warranted. This research should follow a more holistic approach, not only focusing on single exposures, but looking at the totality of environmental exposure from conception onwards [also known as exposomics]. ${ }^{45,52}$

In conclusion, this population-based study in the Dutch South Limburg region showed marked increases in the incidences of adult IBD, CD, and UC between 1991 and 2010. The shift towards milder disease at diagnosis, in parallel with improved diagnostics and ability to detect low-grade inflammation, was suggestive of an important role of diagnostic factors in this increase. Environmental factors probably played a role as well. The mortality was low and, together with the increasing incidence, led to the high prevalence of IBD in South Limburg. 


\section{References}

1. Dignass A, Eliakim R, Magro F, et al. Second European evidence-based consensus on the diagnosis and management of ulcerative colitis part 1 : defnitions and diagnosis. J Crohns Colitis 2012;6:965-90.

2. Van Assche G, Dignass A, Panes J, et al:; European Crohn's and Colitis Organisation [ECCO]. The second European evidence-based consensus on the diagnosis and management of Crohn's disease: defnitions and diagnosis. J Crohns Colitis 2010;4:727.

3. Dignass A, Lindsay JO, Sturm A, et al. Second European evidence-based consensus on the diagnosis and management of ulcerative colitis part 2: current management. J Crohns Colitis 2012;6:991-1030.

4. Dignass A, Van Assche G, Lindsay JO, et al:; European Crohn's and Colitis Organisation [ECCO]. The second European evidence-based consensus on the diagnosis and management of Crohn's disease: current management. J Crohns Colitis 2010;4:28-62.

5. Romberg-Camps MJ, Bol Y, Dagnelie $\mathrm{PC}$, et al. Fatigue and health-related quality of life in inflammatory bowel disease: results from a populationbased study in the Netherlands: the IBD-South Limburg cohort. Inflamm Bowel Dis 2010;16:2137-47.

6. Burisch J, Jess T, Martinato M, Lakatos PL; ECCO-EpiCom. The burden of inflammatory bowel disease in Europe. $J$ Crohns Colitis 2013;7:322-37.

7. Molodecky NA, Soon IS, Rabi DM, et al. Increasing incidence and prevalence of the inflammatory bowel diseases with time, based on systematic review. Gastroenterology 2012;142:4654.e42; quiz e30.
8. Bewtra M, Kaiser LM, TenHave T, Lewis JD. Crohn's disease and ulcerative colitis are associated with elevated standardized mortality ratios: a metaanalysis. Inflamm Bowel Dis 2013;19:599-613.

9. van den Heuvel TR, Pierik MJ, Jonkers DM. On the external validity of epidemiologic data from hospital-based IBD cohorts. J Crohns Colitis 2016;10:1372-3.

10. Kaplan GG. Pitfalls and perils of using administrative databases to evaluate the incidence of inflammatory bowel disease over time. Inflamm Bowel Dis 2014;20:1777-9.

11. Gower-Rousseau C, Savoye G, Colombel JF, Peyrin-Biroulet L. Are we improving disease outcomes in IBD? A view from the epidemiology side. Gut 2014;63:1529-30.

12. Statistics Netherlands. 2015. Http://statline.Cbs.NI. Accessed December 2015.

13. van den Heuvel TR, Jonkers DM, Jeuring SF, et al. Cohort Profle: The inflammatory bowel disease South Limburg cohort [IBDSL]. Int J Epidemiol 2015, Jun 4. pii: dyv088.

14. Casparie M, Tiebosch AT, Burger $G$, et al. Pathology databanking and biobanking in The Netherlands, a central role for PALGA, the nationwide histopathology and cytopathology data network and archive. Cell Oncol 2007;29:19-24.

15. World Medical Association. World Medical Association Declaration of Helsinki: ethical principles for medical research involving human subjects. JAMA 2013;310:2191-4.

16. Lennard-Jones JE. Classifcation of inflammatory bowel disease. Scand J 
Gastroentero/ Supp/ 1989;170:2-6;

discussion 16-9.

17. Satsangi J, Silverberg MS, Vermeire S, Colombel JF. The Montreal classifcation of inflammatory bowel disease: controversies, consensus, and implications. Gut 2006;55:749-53.

18. Kim HJ, Fay MP, Feuer EJ, Midthune DN. Permutation tests for joinpoint regression with applications to cancer rates. Stat Med 2000;19:335-51.

19. Clegg LX, Hankey BF, Tiwari R, Feuer EJ, Edwards BK. Estimating average annual per cent change in trend analysis. Stat Med 2009;28:3670-82.

20. Kim HJ, Fay MP, Yu B, Barrett MJ, Feuer EJ. Comparability of segmented line regression models. Biometrics 2004;60:1005-14.

21. Ahmad OB, Boschi-Pinto C, Lopez AD. Age Standardization of Rates: A New WHO Standard. Geneva: World Health Organization; 2001.

22. GBA-V.

2015.Http://www.Rijksdienstvooridenti teitsgegevens.NI. Accessed December 2015.

23. Koloski NA, Bret $L$, Radford-Smith $G$. Hygiene hypothesis in inflammatory bowel disease: a critical review of the literature. World J Gastroenterol 2008;14:165-73.

24. Spooren CE, Pierik MJ, Zeegers MP, Feskens EJ, Masclee AA, Jonkers DM. Review article: the association of diet with onset and relapse in patients with inflammatory bowel disease. Aliment Pharmacol Ther 2013;38:1172-87.

25. Kaplan GG, Hubbard J, Korzenik J, et al. The inflammatory bowel diseases and ambient air pollution: a novel association. Am J Gastroenterol 2010;105:2412-9.

26. Soon IS, Molodecky NA, Rabi DM, Ghali WA, Barkema HW, Kaplan GG. The relationship between urban environment and the inflammatory bowel diseases: a systematic review and meta-analysis. BMC Gastroenterol 2012;12:51.

27. Ananthakrishnan AN, McGinley EL, Binion DG, Saeian K. Ambient air pollution correlates with hospitalizations for inflammatory bowel disease: an ecologic analysis. Inflamm Bowe/ Dis 2011;17:1138-45.

28. Salim SY, Kaplan GG, Madsen KL. Air pollution effects on the gut microbiota: a link between exposure and inflammatory disease. Gut Microbes 2014;5:215-9.

29. Vind I, Riis L, Jess T, et al:; DCCD study group. Increasing incidences of inflammatory bowel disease and decreasing surgery rates in Copenhagen City and County, 20032005: a population-based study from the Danish Crohn Colitis database. Am J Gastroenterol2006;101:1274-82.

30. Sjöberg D, Holmström T, Larsson M, et al. Incidence and clinical course of Crohn's disease during the frst yearresults from the IBD Cohort of the Uppsala Region [ICURE] of Sweden 2005-2009. J Crohns Colitis 2014;8:215-22.

31. Sjöberg D, Holmström T, Larsson M, et al. Incidence and natural history of ulcerative colitis in the Uppsala Region of Sweden 2005-2009-results from the IBD cohort of the Uppsala Region [ICURE]. J Crohns Colitis 2013;7:e351-7.

32. Gower-Rousseau C, Vasseur F, Fumery $M$, et al. Epidemiology of inflammatory bowel diseases: new insights from a French population-based registry [EPIMAD]. Dig Liver Dis 2013;45:8994.

33. Sivak MV. Gastrointestinal endoscopy: past and future. Gut 2006;55:1061-4.

34. Jeuring SF, van den Heuvel TR, Liu LY, et al. Improvements in the longterm outcome of Crohn's disease over the 
past two decades and the relation to changes in medical management: results from the population-based IBDSL cohort. Am J Gastroenterol 2017;112:325-36.

35. Jeuring SF, Bours PH, Zeegers MP, et al. Disease outcome of ulcerative colitis in an era of changing treatment strategies: results from the Dutch population-based IBDSL cohort. J Crohns Colitis 2015;9:837-45.

36. Halfvarson J, Bodin L, Tysk C, Lindberg E, Järnerot G. Inflammatory bowel disease in a Swedish twin cohort: a long-term follow-up of concordance and clinical characteristics.

Gastroenterology 2003;124:1767-73.

37. Tysk C, Lindberg E, Järnerot G, Flodérus-Myrhed B. Ulcerative colitis and Crohn's disease in an unselected population of monozygotic and dizygotic twins. A study of heritability and the influence of smoking. Gut 1988;29:990-6.

38. Orholm M, Binder V, Sørensen TI, Rasmussen LP, Kyvik KO. Concordance of inflammatory bowel disease among Danish twins. Results of a nationwide study. Scand J Gastroenterol 2000;35:1075-81.

39. Thompson AJ, Reid AJ, Reid M. Congenital lobar emphysema occurring in twins. J Perinat Med 2000;28:1557.

40. Pinsk V, Lemberg DA, Grewal K, Barker CC, Schreiber RA, Jacobson K. Inflammatory bowel disease in the South Asian pediatric population of British Columbia. Am J Gastroenterol 2007; 102:1077-83.

41. Probert CS, Jayanthi V, Pinder D, Wicks AC, Mayberry JF. Epidemiological study of ulcerative proctocolitis in Indian migrants and the indigenous population of Leicestershire. Gut 1992;33:68793.
42. Barreiro-de Acosta M, Alvarez Castro A, Souto R, Iglesias M, Lorenzo A, Dominguez-Munoz JE. Emigration to Western industrialised countries: A risk factor for developing inflammatory bowel disease. J Crohns Colitis 2011;5:566-9.

43. Ng SC, Bernstein CN, Vatn MH, et al:; Epidemiology and Natural History Task Force of the International Organization of Inflammatory Bowel Disease [IOIBD]. Geographical variability and environmental risk factors in inflammatory bowel disease. Gut 2013;62:630-49.

44. Rogler G, Zeitz J, Biedermann L. The search for causative environmental factors in inflammatory bowel disease. Dig Dis 2016:34[Suppl 1]:48-55.

45. Rogler $G$, Vavricka S. Exposome in IBD: recent insights in environmental factors that influence the onset and course of IBD. Inflamm Bowel Dis 2015;21:4008.

46. Maaser C, Langholz E, Gordon H, et al. European Crohn's and Colitis Organisation topical review on environmental factors in IBD. J Crohns Colitis 2016, Dec 30. pii: jjw223. [Epub ahead of print.]

47. Green C, Elliott L, Beaudoin C, Bernstein CN. A population-based ecologic study of inflammatory bowel disease: searching for etiologic clues. Am J Epidemiol2006; 164:615-23; discussion 624-8.

48. Jeuring SF, van den Heuvel TR, Zeegers $M P$, et al. Epidemiology and long-term outcome of inflammatory bowel disease diagnosed at elderly age - an increasing distinct entity? Inflamm Bowel Dis 2016:22:1425-34.

49. Thun MJ, Carter BD, Feskanich D, et al. 50-year trends in smoking-related mortality in the United States. N Eng/ J Med 2013;368:351-64. 
50. Steenbakkers M, Vermeer AJM, Janssen-Goffn MJH, Hajema KJ. Een nieuwe kijk op gezondheid in zuidlimburg. Regionaal rapport volksgezondheid toekomst verkenning 2014 [A new perspective on health in South Limburg. Regional report 2014]. Geleen, The Netherlands: GGD ZuidLimburg, 2014.

51. Jansen M, Kuppens E. Op zoek naar de limburg factor [Searching for the Limburg factor]. Limburg, The Netherlands: Provincie Limburg \& GGD Zuid Limburg, 2015.

52. Rappaport AM. An individual perspective on risk in a DC [usually 401[k]] environment. Benefts $Q$ 2016;32:8-14 



\section{Abstract}

\section{Background}

Elderly onset [EO] inflammatory bowel disease [IBD] may become a more common entity as a result of population aging and the rising IBD incidence. Its management is challenging, because of multimorbidity, polypharmacy, and frailty. Insight into the long-term outcome is essential for optimal patient counseling and treatment. We studied the incidence and disease outcome of elderly-onset IBD in direct comparison to adult-onset [AO] IBD.

\section{Methods}

All 2823 cases with IBD from the Dutch population-based IBD South Limburg cohort, diagnosed between 1991 and 2011, were included. Long-term outcome [hospitalization, surgery, and disease phenotype] was compared between $\mathrm{AO}$ [ $<60$ years at diagnosis] and EO [ $\geq 60$ years at diagnosis] disease, for Crohn's disease [CD] and ulcerative colitis [UC] separately.

\section{Results}

In total, 1162 patients with CD [136 EO/1026 AO] and 1661 patients with UC [373 EO/1288 AO] were included. The EO IBD incidence increased from 11.71 per 100,000 persons in 1991 to 23.66 per 100,000 persons in 2010, $p<0.01$. Immunomodulators were less often used in EO CD [61.8\% versus $77.1 \%, p=0.03]$ and EO UC [22.8\% versus 35.4\%, $p<0.01$ ], even as biologicals [25.1\% versus 55.1\%, $p=0.03$ and $7.8 \%$ versus $18.0 \%$, $p<0.01$, respectively]. No differences were observed in surgery risk [CD: hazard ratio [HR] 1.19; 95\% confidence interval [Cl], 0.85-1.67 and UC: HR, 0.88; 95\% Cl, 0.53-1.46], or in CD phenotype progression [HR, 0.81; 95\% Cl, 0.52-1.25], but more patients with EO UC required hospitalization [HR, 1.29; 95\% Cl, 1.01-1.63].

\section{Conclusions}

EO IBD is rising, warranting physicians' alertness for IBD in elderly patients. The long-term outcome was not different from $\mathrm{AO}$ disease, despite a less frequent use of immunomodulators and biologicals. 


\section{Introduction}

Inflammatory bowel disease [IBD] can develop at any age, but has a peak incidence in young adulthood. A second incidence peak at older age has been observed in ulcerative colitis [UC] in some studies. ${ }^{1-10}$ Pooled data from European population-based studies, conducted in the 1990s, report an incidence of 2.5 per 100,000 for Crohn's disease [CD] and 8.1 per 100,000 for UC in the elderly population. ${ }^{11}$ Studies from North America and Canada have found slightly higher incidence rates in their elderly populations. ${ }^{6,12,13}$ Recent data showed that the incidences of both CD and UC have increased over the last decades and are still increasing. 9,10 However, recent data on the evolution of the IBD incidence rate in the elderly population are scarce.

Population aging is a demographic phenomenon challenging many Western countries. ${ }^{14}$ In the Netherlands, the overall proportion of elderly increased from $11.5 \%$ to $17.4 \%$ between 1980 and $2014 .{ }^{15}$ Demographic models indicate that this number is expected to increase toward approximately $26 \%$ in the upcoming decades. ${ }^{16}$ Population aging will inevitably lead to a higher number of elderly patients with IBD in our clinics, warranting information on the question whether the outcome of elderly-onset [EO] IBD is similar to adultonset $[\mathrm{AO}]$ IBD or that EO IBD must be considered a distinct entity.

Current treatment guidelines make no distinction between patients at adult or elderly age. ${ }^{17,18}$ The use of immunomodulating drugs is associated with a higher prevalence of specific types of cancer and [opportunistic] infections, the latter particularly if given as combination therapy with anti-tumor necrosis factor $\alpha$ [TNF $\alpha$ ] agents or systemic corticosteroid [CS] therapy. ${ }^{19-21}$ In the TREAT registry, as well as in an Italian multicenter study, older age was associated with a higher risk of serious infections and mortality in patients with IBD on antiTNF $\alpha$ therapy. ${ }^{22,23}$ In general, elderly are at increased risk for developing infections and for a more severe course of the infection. ${ }^{24}$ Therefore, caution in drug prescription is warranted, in particular in the frail elderly population. ${ }^{25-27}$

Data on the disease course of EO IBD are mainly derived from small selected referral center cohorts, some collected decades ago. ${ }^{28-39}$ Recent population-based data from the Hungarian registry and the French EPIMAD registry suggested a somewhat milder course of IBD diagnosed at older age, yet the number of elderly patients was limited ${ }^{40}$ or data from the corresponding adult population were lacking, ${ }^{41}$ impeding a direct comparison. To balance benefit and risk of treatment strategies in the elderly population, information on the current treatment strategy and disease outcome is needed, preferably from a large unselected population of patients with IBD studied in direct comparison to AO disease.

The aim of this study was to assess the incidence of IBD diagnosed at elderly age in a large Dutch population-based cohort, and to directly compare the long-term outcome to the corresponding adult IBD population. 


\section{Methods}

\section{Study population}

The IBD South Limburg [IBDSL] cohort is a population-based IBD cohort in the South Limburg area of the Netherlands. Since 1991, patients with incident IBD in this well-defined geographical area are being included in the IBDSL registry. A recent case ascertainment check showed that more than 93\% of all adult patients with IBD living in the South Limburg area and diagnosed since 1991 are registered in the cohort. For detailed information on the IBDSL cohort, we refer to the cohort profile. ${ }^{42}$

All patients with CD or UC present in the IBDSL registry were included. These patients were diagnosed between January 1, 1991 and December 31, 2010 [UC] and July 2011 $[C D]$ at an age of 18 years or older. Each patient was followed from the date of diagnosis to the end of current data collection [2011 for UC and 2014 for CD], date of migration out of the area, or death. Medical records were thoroughly reviewed with regard to demographic data, disease extent and location, disease behavior, medication use, hospitalization, and surgery. All data were extracted from the medical records using standardized registration forms.

This study has been approved by the Medical Ethics Committee of the Maastricht University Medical Center [NL31636.068.10] and the IBDSL cohort is registered in ClinicalTrial.gov [NCT02130349].

\section{Study design and end points}

Within the IBDSL population, 2 patient groups were defined, based on the age at diagnosis: $\mathrm{AO}$ IBD [age at diagnosis between 18 and 60 years] and EO IBD [age at diagnosis 60 years or older]. The cutoff value for elderly age [60 years] was chosen in line with previous literature on this topic, ${ }^{40,41}$ facilitating data comparison.

Primary endpoints were the incidence of EO IBD and the long-term disease outcome. Disease outcome was studied in terms of disease progression, the occurrence of an IBDrelated hospitalization, and the occurrence of surgery. The use of immunomodulators or antiTNF $\alpha$ agents was not considered a disease outcome parameter, as their use may be confounded in the elderly by polypharmacy, therapy risks, and personal preferences from either physician or patient on drug use at older age. Disease progression in CD was defined according to the Montreal classification, as progression from non-stricturing, non-penetrating disease [B1] to stricturing [B2] or penetrating disease [B3] ${ }^{43-45}$ For UC, disease progression was defined as progression to a more proximal colon location, i.e. E1 [proctitis] to E2 [leftsided disease] or E3 [extensive disease] or E2 to E3 ${ }^{43}$ Hospitalization was defined as a hospital admission for IBD-related complaints [first presentation or flare], IBD-related surgery, or a combination of both. Hospital admissions for drug administration or elective endoscopies 
were excluded. Surgery was defined as resection of a part of the bowel because of [refractory] active disease or IBD-related complications, such as stricturing or penetrating disease, or toxic megacolon. The endpoints were determined at diagnosis [defined as within 1 month after diagnosis] and during follow-up [at 5 and 10 years after diagnosis] to discriminate between disease presentation and long-term disease outcome.

Secondary endpoint was IBD medication use. In these analyses, treatment failure was defined as treatment cessation following no response or loss of response as indicated by the treating physician. Dates of treatment initiation and cessation were retrieved from the medical files. In the assessment of treatment duration, a temporary treatment stop [defined as shorter than 3 months] and a switch to another drug from the same class [e.g. switch from azathioprine to mercaptopurine] were not considered as treatment failure. Data on the cumulative prednisone exposure were only available in patients with $C D$, and analyses were performed in patients with a minimum of 12 months of follow-up.

\section{Statistical analyses}

Data were presented as means with SDs or as medians with interquartile ranges [IQRs], depending on normality of the underlying distribution. Continuous data were compared by the independent Student's t test [normal distribution] or Mann-Whitney $U$ test [nonparametric distribution]. Categorical data were compared by the chi-square test. The incidence of IBD was stratified by sex, age, and year of diagnosis and subsequently expressed as the incidence rate of AO IBD and EO IBD per 100,000 inhabitants of the South Limburg area. Time trends in incidence rate were assessed by linear regression. Kaplan-Meier survival analyses were undertaken for events that developed during disease course, such as hospitalization, surgery, initiation of a specific medical treatment, and the development of strictures or fistulas/abscesses [CD] or proximal extension of disease location [UC]. A multivariable Cox regression model was used to assess the association between $\mathrm{AO}$ or EO disease and the occurrence of the event of interest, while correcting for known clinical confounders or differences in baseline characteristics between groups. In all CD models, sex, disease location, disease behavior, and the presence of perianal and upper gastrointestinal disease at diagnosis were additionally included. In all UC models, sex and disease extents at diagnosis were additionally included. Differences in hazards between groups were expressed as hazard ratios [HRs] with accompanying 95\% confidence intervals [95\%Cls]. AO disease was used as reference category in all analyses. The proportional hazards' assumption was verified for every variable, by testing significance of the interaction term of every parameter with time. Two-sided $p$ values lower than 0.05 were considered statistically significant. All statistical analyses were performed using IBM SPSS Statistics for Windows, Version 22.0 [IBM Corp., Armonk, NY]. 


\section{Results}

\section{Study population}

In total, 1162 patients with CD and 1661 patients with UC were diagnosed between January 1991 and December 2010 [UC] and July 2011 [CD] in the South Limburg area. IBD diagnosis was established above the age of 60 in 136 [11.7\%] patients with CD and 373 [22.5\%] patients with UC.

Patient characteristics are shown in Tables 4.1 and 4.2. Median follow-up was 8.0 years [IQR, 4.3-12.9] in AO CD and 5.6 years [IQR, 2.8-8.9] in patients with EO CD. For UC, median follow-up was 9.0 years [IQR, 4.6-15.2] in AO and 7.1 years [IQR, 3.7-13.5] in EO patients. In CD, ileocolonic disease was less often observed in $\mathrm{EO}$ patients than in $\mathrm{AO}$ patients $[p=0.01]$. In UC, a similar number of patients had extensive disease at diagnosis, but more left-sided than rectal disease was seen in EO UC [ $p=0.01]$.

Table 4.1. Patient characteristics of adult-onset and elderly-onset CD patients in the IBDSL cohort

\begin{tabular}{|c|c|c|c|c|}
\hline & & $\begin{array}{c}\text { Adult-onset CD } \\
{[\mathrm{N}=1026]}\end{array}$ & $\begin{array}{c}\text { Elderly-onset CD } \\
{[\mathrm{N}=136]}\end{array}$ & \\
\hline Age at diagnosis & mean [SD] & $33.5[11.5]$ & $69.1[7.0]$ & $p<0.01$ \\
\hline Sex - male & $N[\%]$ & $384[37.4]$ & $50[36.8]$ & $p=0.93$ \\
\hline Follow-up in years & median [IQR] & $8.0[4.3-12.9]$ & $5.6[2.8-8.9]$ & $p<0.01$ \\
\hline Disease location at diagnosis & & & & $p<0.01$ \\
\hline L1, ileal location & $N[\%]$ & $431[42.0]$ & $69[50.7]$ & \\
\hline L2, colon location & $N[\%]$ & $322[31.4]$ & $49[36.0]$ & \\
\hline L3, ileocolon location & $N[\%]$ & $254[24.8]$ & $13[9.6]$ & \\
\hline$\llcorner 4$, upper GI only & $N[\%]$ & $19[1.9]$ & $5[3.7]$ & \\
\hline Disease behavior at diagnosis & & & & $p=0.77$ \\
\hline B1 - inflammatory & $N[\%]$ & $845[82.3]$ & $112[82.3]$ & \\
\hline B2 - stricturing & $N[\%]$ & $121[11.8]$ & 18 [13.2] & \\
\hline B3 - penetrating & $N[\%]$ & $60[5.9]$ & $6[4.5]$ & \\
\hline Perianal disease at diagnosis & $N[\%]$ & 89 [8.7] & 5 [3.7] & $p=0.04$ \\
\hline Upper GI location at diagnosis & $N[\%]$ & $103[10.0]$ & $21[15.4]$ & $p=0.07$ \\
\hline
\end{tabular}

$\mathrm{SD}$, standard deviation; IQR, interquartile range; GI, gastrointestinal

\section{Incidence of EO IBD}

Between 1991 and 2010, the incidence rate of AO IBD increased from 19.26 to 46.14 per 100,000 and the incidence rate of EO IBD increased from 11.71 to 23.66 per 100,000, $p=$ 0.01 for both. For $A O C D$, the incidence rate increased from 6.84 to 22.78 per 100,000 and the incidence rate of EO CD from 2.51 to 6.23 per 100,000 [Figure 4.1], $p=0.01$ for both. In UC, the incidence rate of $A O$ disease increased from 12.42 to 23.36 per 100,000 


\begin{tabular}{|c|c|c|c|c|}
\hline & & $\begin{array}{l}\text { Adult-onset UC } \\
\quad[N=1288]\end{array}$ & $\begin{array}{c}\text { Elderly-onset UC } \\
{[\mathrm{N}=373]}\end{array}$ & \\
\hline Age at diagnosis & mean $[S D]$ & $38.9[11.5]$ & $69.6[6.8]$ & $p<0.01$ \\
\hline Sex - male & $N[\%]$ & 673 [52.3] & 212 [56.8] & $p=0.13$ \\
\hline Follow-up in years & median [IQR] & $9.0[4.6-15.2]$ & $7.1[3.7-13.5]$ & $p<0.01$ \\
\hline Disease location at diagnosi & & & & $p<0.01$ \\
\hline E1, proctitis & $N[\%]$ & $465[36.1]$ & 98 [26.3] & \\
\hline E2, left-sided disease & $\mathrm{N}[\%]$ & 578 [44.9] & $211[56.6]$ & \\
\hline E3, extensive disease & $N[\%]$ & $233[18.1]$ & $63[16.9]$ & \\
\hline
\end{tabular}

$\mathrm{SD}$, standard deviation; IQR, interquartile range

and the incidence rate of EO disease increased from 9.20 to 17.43 per 100,000 [Figure 4.1], $p=0.01$ for both. In addition, the proportion of elderly in newly diagnosed patients was determined. In CD, this proportion was fairly stable over time: $10.0 \%$ in 1991 and $11.4 \%$ in 2010, $p=0.45$. In UC, on the contrary, the proportion of elderly increased from $18.3 \%$ in 1991 to $25.9 \%$ in $2010, p=0.01$.
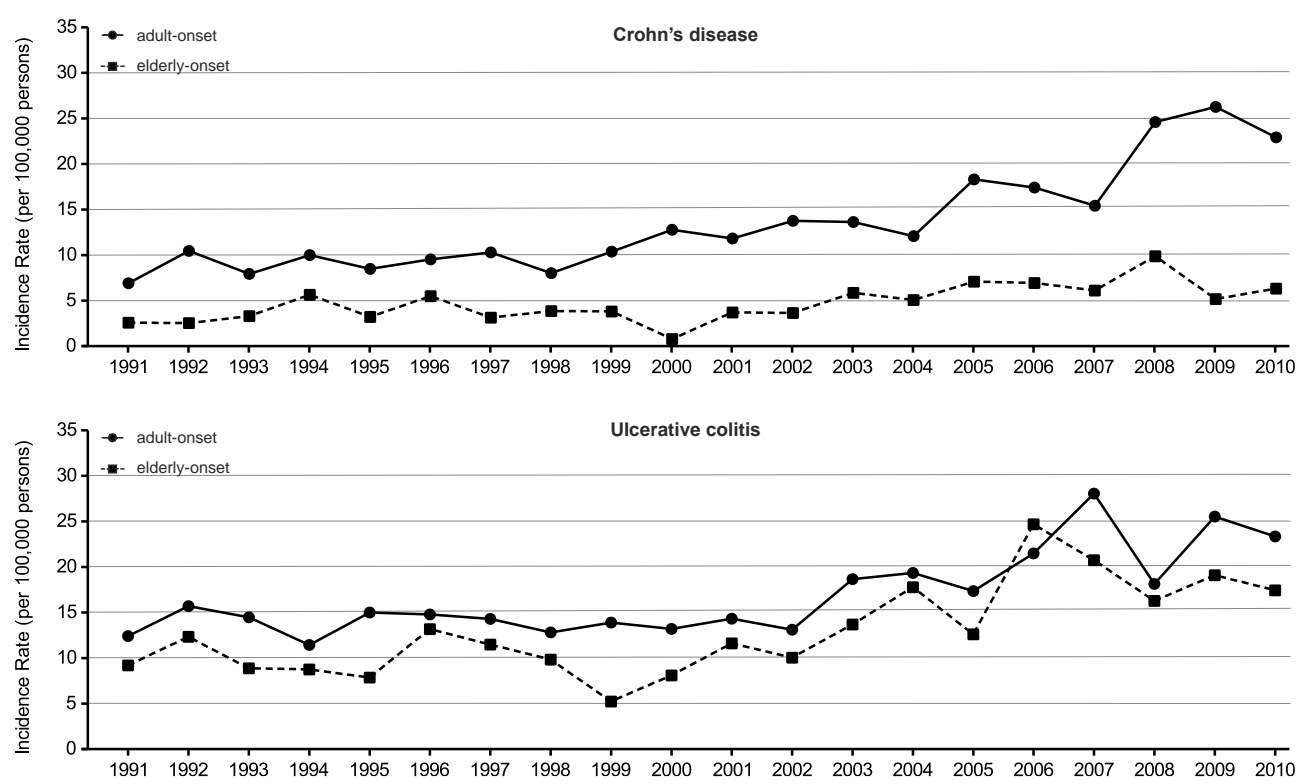

Figure 4.1. Incidence rates of adult-onset and elderly-onset Crohn's disease and ulcerative colitis in the South Limburg area of the Netherlands. 


\section{Medication use}

Systemic CS therapy was ever used by 647 [58.6\%] patients with CD and 726 [44.9\%] patients with UC. In CD, EO disease was associated with a lower use of CS therapy, compared with $\mathrm{AO}$ disease [38.7\% versus $61.0 \%$; adjusted $\mathrm{HR}, 0.45 ; 95 \% \mathrm{Cl}, 0.30-0.69$ ]. During the disease course, patients with CD who were ever treated with CS used a median of 21.3 [IQR, 10.3-47.7] days prednisone per patient-year, but this did not differ significantly between EO $\mathrm{CD}$ and $\mathrm{AO} C \mathrm{CD}$ [median, 22.1 [IQR, 10.5-47.8] versus median, 15.5 [IQR, 9.1-46.3] days per patient-year, $p=0.85]$. In addition, the 647 patients with $C D$ were exposed to a median of 427.4 [IQR, 208.1-810.1] mg of prednisone per patient-year, being similar for EO and AO CD [median, 433.8 [IQR, 208.6-811.7] mg versus median, 329.8 [IQR, 201.0-840.3] mg, $p=0.61$. In UC, no difference regarding ever CS use was observed between $A O$ and EO diseases [45.9\% versus 45.3\%; adjusted $\mathrm{HR}, 0.95 ; 95 \% \mathrm{Cl}, 0.75-1.22$ ]. For this group, data on cumulative prednisone use were not available for study.

Immunomodulator therapy was prescribed in 696 [63.0\%] patients with $C D$ and in 390 [24.6\%] patients with UC. The cumulative exposure to immunomodulator treatment is shown in Figure 4.2A [CD] and Figure 4.2B [UC]. Both patients with EO CD and EO UC less often used immunomodulator treatment in comparison to the corresponding $\mathrm{AO}$ patients [adjusted HR, 0.74; 95\% Cl, 0.56-0.97 and adjusted HR, 0.66; 95\% Cl, 0.50-0.87, respectively]. Neither in $\mathrm{CD}$, nor in $U \mathrm{C}$, immunomodulator discontinuation rate differed between $\mathrm{AO}$ and $\mathrm{EO}$ disease [cumulative 10-year probability of $75.5 \%$ versus $81.6 \%$, adjusted $\mathrm{HR}, 1.17 ; 95 \% \mathrm{Cl}, 0.82-1.66$ in $\mathrm{CD}$ and cumulative 10 -year probability of $64.5 \%$ versus $66.0 \%$, adjusted $\mathrm{HR}, 0.98 ; 95 \% \mathrm{Cl}, 0.66-1.47$ in $\mathrm{UC}]$. Reasons for treatment cessation were combined for $C D$ and $U C$ and are shown in Table 4.3. Side effects were the most common reason for treatment cessation for immunomodulator therapy in both $\mathrm{AO}$ [50.9\%] and EO [64.3\%] patients, $p=0.06$.

Biological therapy was more frequently used in patients with $C D$ than in patients with UC [34.2\% versus $9.2 \%]$. The cumulative probability of starting biological therapy is shown in Figure 4.2C [CD] and Figure 4.2D [UC]. In total, 20 patients with EO CD and 15 patients with EO UC ever used anti-TNF $\alpha$ therapy. Patients with EO CD 1.6 times less often used biological therapy in comparison to patients with $\mathrm{AO} C \mathrm{CD}$ [adjusted $\mathrm{HR}, 0.61 ; 95 \% \mathrm{Cl}, 0.38-0.96$ ] and patients with EO UC 2.4 times less often used biological therapy in comparison to patients with $\mathrm{AO} \cup \mathrm{C}$ [adjusted $\mathrm{HR}, 0.42 ; 95 \% \mathrm{Cl}, 0.25-0.72$ ]. No significant difference in therapy cessation rate was observed in CD [29.4\% in EO CD versus $59.4 \%$ in $A O C D$; adjusted HR, $0.71 ; 95 \% \mathrm{Cl}, 0.33-1.53]$, nor in UC [67.0\% in EO UC and $40.0 \%$ in AO UC; adjusted HR, $1.25 ; 95 \% \mathrm{Cl}, 0.53-2.94]$. Reasons for treatment cessation were combined for $\mathrm{CD}$ and $\mathrm{UC}$ and are shown in Table 4.3. Loss of response was the most common reason for the discontinuation of anti-TNF $\alpha$ agents in both $\mathrm{AO}[40.1 \%]$ and EO patients [46.2\%], $p=0.11$. 
A

\section{Cumulative use of immunomodulators}

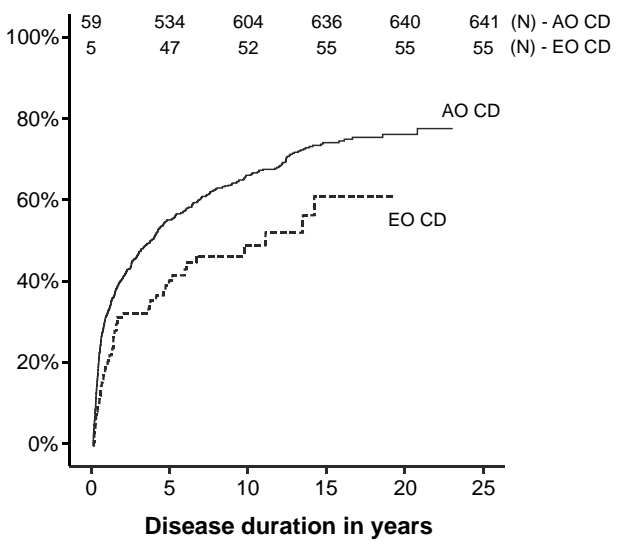

$\begin{array}{lcccccc}\text { No at risk } 986 & 332 & 164 & 71 & 20 & 0 & \text { AO CD } \\ \text { No at risk } 119 & 46 & 19 & 7 & 0 & 0 & \text { EO CD }\end{array}$

C

\section{Cumulative use of anti-TNFá agents}

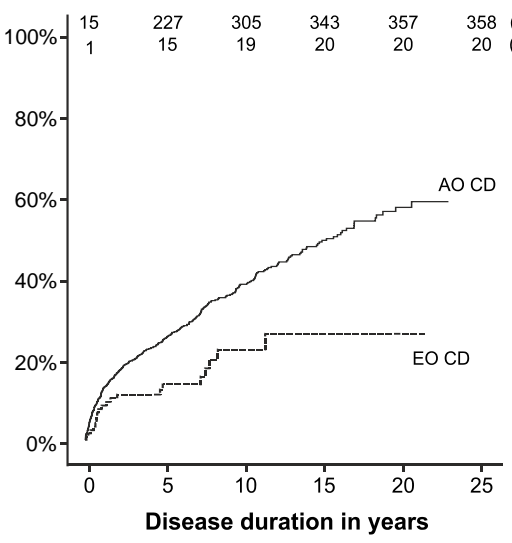

$\begin{array}{lcccccc}\text { No at risk } 986 & 562 & 291 & 130 & 36 & 0 & \text { AO CD } \\ \text { No at risk } 119 & 70 & 26 & 10 & 1 & 0 & \text { EO CD }\end{array}$
B

\section{Cumulative use of immunomodulators}

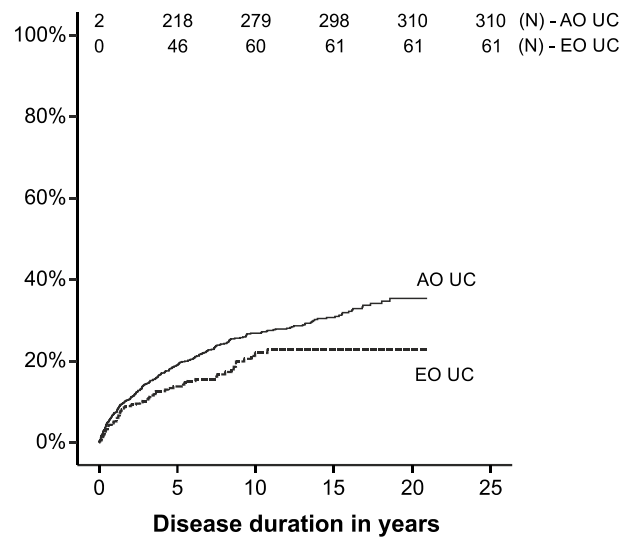

$\begin{array}{lllllll}\text { No at risk } 1226 & 739 & 448 & 247 & 38 & 0 & \text { AO UC }\end{array}$ $\begin{array}{lllllll}\text { No at risk } 358 & 207 & 108 & 63 & 10 & 0 & \text { EO UC }\end{array}$

D

\section{Cumulative use of anti-TNFá agents}

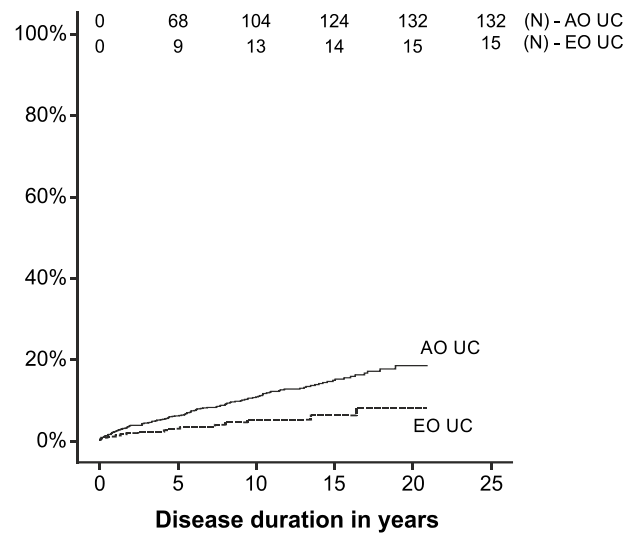

$\begin{array}{lllllll}\text { No at risk } 1226 & 881 & 560 & 300 & 45 & 0 & \text { AO UC }\end{array}$ $\begin{array}{lllllll}\text { No at risk } 358 & 238 & 132 & 69 & 10 & 0 & \text { EO UC }\end{array}$

Figure 4.2. Cumulative probability of using immunomodulators [A and B] and anti-TNF $\alpha$ agents [C and D] in adultonset and elderly-onset IBD

CD, Crohn's disease; $U C$, ulcerative colitis; $A O$, adult-onset; $E O$, elderly-onset; $\mathrm{N}$, number of patients with specific drug use 


\begin{tabular}{|c|c|c|c|c|c|c|c|}
\hline & & $\begin{array}{c}\mathrm{AO} \text { IBD } \\
{[\mathrm{N}=951]}\end{array}$ & $\begin{array}{c}E O \text { IBD } \\
{[N=116]}\end{array}$ & & $\begin{array}{c}\mathrm{AO} \text { IBD } \\
{[\mathrm{N}=490]}\end{array}$ & $\begin{array}{l}\text { EO IBD } \\
{[N=35]}\end{array}$ & \\
\hline Cessation & $N[\%]$ & $629[66.1]$ & $70[60.3]$ & $p=0.06^{b}$ & $257[52.4]$ & $13[37.1]$ & $p=0.11^{b}$ \\
\hline \multicolumn{8}{|l|}{ Reason for cessation } \\
\hline Quiescent disease & $N[\%]$ & $122[19.4]$ & $7[10.0]$ & & 37 [14.4.] & 0 & \\
\hline Loss of response & $N[\%]$ & $62[9.9]$ & $5[7.1]$ & & $103[40.1]$ & $6[46]$. & \\
\hline Patient & $N[\%]$ & $57[9.1]$ & $3[4.3]$ & & $13[5.1]$ & $1[7.7]$ & \\
\hline Trough levels & $N[\%]$ & $12[1.9]$ & $1[1.4]$ & & - & - & \\
\hline Pregnancy & $\mathrm{N}[\%]$ & 14 [2.2] & 0 & & $8[3.1]$ & 0 & \\
\hline Side effects & $N[\%]$ & $320[50.9]$ & 45 [64.3] & & 82 [31.9] & $3[23.1]$ & \\
\hline Gl intolerance & $N[\%]$ & 101 & 8 & & 0 & 0 & \\
\hline Malaise & $N[\%]$ & 15 & 4 & & 2 & 0 & \\
\hline Pancreatitis & $N[\%]$ & 60 & 8 & & 0 & 0 & \\
\hline Liver enzymes & $N[\%]$ & 37 & 12 & & 0 & 0 & \\
\hline Leukopenia & $N[\%]$ & 25 & 3 & & 0 & 0 & \\
\hline Skin rash & $N[\%]$ & 24 & 1 & & 13 & 0 & \\
\hline Fever & $N[\%]$ & 17 & 3 & & 0 & 0 & \\
\hline Joints & $N[\%]$ & 12 & 0 & & 11 & 0 & \\
\hline Infusion reaction & $N[\%]$ & - & - & & 30 & 0 & \\
\hline $\begin{array}{l}\text { Delayed } \\
\text { hypersensitivity }\end{array}$ & $N[\%]$ & - & - & & 9 & 1 & \\
\hline Other & $N[\%]$ & 29 & 6 & & 17 & 2 & \\
\hline Other reason & $N[\%]$ & $42[6.7]$ & 9 [12.9] & & $14[5.4]$ & $3[23.1]$ & \\
\hline
\end{tabular}

$\mathrm{AO}$, adult-onset; $\mathrm{EO}$, elderly-onset; $\mathrm{Gl}$, gastrointestinal

${ }^{a}$ for these analyses, treatment cessation results were combined for Crohn's disease and ulcerative colitis

${ }^{b}$ Chi-square analyses were performed between groups with the total number of side effects 


\section{Disease outcome: disease phenotype}

The distribution of disease phenotypes in CD is shown in Figure 4.3. At diagnosis, phenotype distribution was not different between patients with $\mathrm{EO}$ and $\mathrm{AO}$ CD: $82.3 \%$ and $82.3 \%$ had B1 phenotype, $13.2 \%$ and $11.8 \%$ had B2 phenotype, and $4.5 \%$ and $5.9 \%$ had B3 phenotype at that time, $p=0.72$. The 5-year and 10-year cumulative probabilities of disease progression to either B2 or B3 phenotype were $24.2 \%$ and $33.5 \%$ in AO CD and 19.8\% and 29.9\% in EO $\mathrm{CD}$, adjusted $\mathrm{HR}, 0.81 ; 95 \% \mathrm{Cl}, 0.52-1.25$. Progression from ileal or colonic to ileocolonic localization was observed in 182 patients [15.6\%], being lower in EO [6.6\%] than in AO [16.9\%] CD [ $p=0.01]$. In UC, proximal extension to either left-sided or extensive disease location occurred in 12.0\% [AO] and 12.3\% [EO] within 5 years and in 19.9\% [AO] and 20.8\% [EO] within 10 years, $p=0.79$. Other parameters associated with phenotype progression are shown in Tables 4.S1 and 4.S2.

A

\section{Adult-onset Crohn's disease}

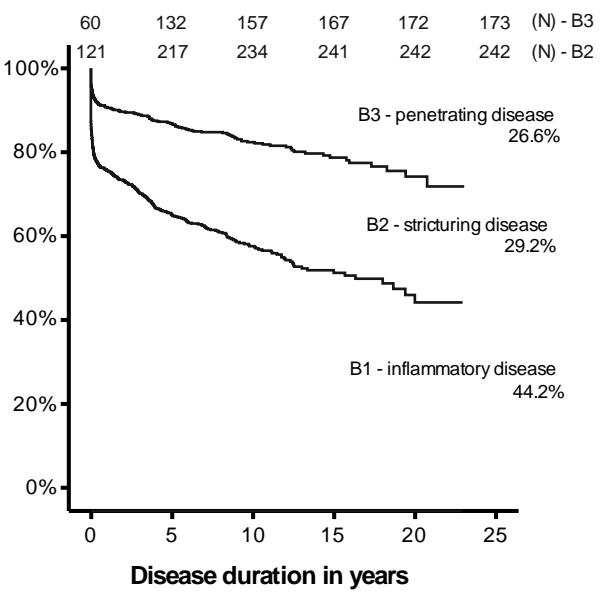

$\begin{array}{llllll}\text { No at risk } & 1026 & 443 & 211 & 81 & 24\end{array}$
B

\section{Elderly-onset Crohn's disease}

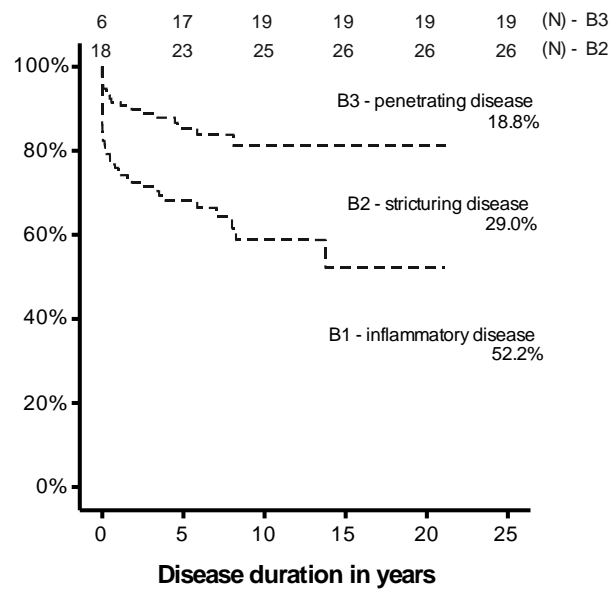

No at risk 136

0

Figure 4.3. Disease phenotype distribution according to the Montreal classification in adult-onset [A] and elderlyonset [B] Crohn's disease.

$\mathrm{N}$, number of patients with specific disease phenotype 


\section{Disease outcome: hospitalization}

The cumulative probability of an IBD-related hospitalization is shown in Figure 4.4A [CD] and Figure 4.4B [UC]. More patients with CD than UC ever needed hospital admission for IBD [55.2\% versus $21.2 \%$, respectively]. In UC, more patients with EO than $A O$ were hospitalized at the time of diagnosis [5.7\% versus 2.9\%, adjusted $\mathrm{HR}, 1.89 ; 95 \% \mathrm{Cl}, 1.10-3.24$ ], whereas this rate was similar in $\mathrm{CD}$ [30.2\% versus $25.3 \%$, adjusted $\mathrm{HR}, 1.14 ; 95 \% \mathrm{Cl}, 0.82-1.58]$. Overall, no difference in hospitalization risk was observed between age groups in CD [adjusted $\mathrm{HR}, 1.02 ; 95 \% \mathrm{Cl}, 0.78-1.32$ ], whereas a higher hospitalization risk was observed in patients with EO UC during follow-up [adjusted HR, 1.29; 95\% Cl, 1.01-1.63]. Many patients required a subsequent hospitalization [56.8\% in CD and $51.2 \%$ in UC], although no differences were observed between $\mathrm{AO}$ and $\mathrm{EO}$ disease [adjusted $\mathrm{HR}, 0.97 ; 95 \% \mathrm{Cl}, 0.62-1.51$ and adjusted $\mathrm{HR}, 0.91 ; 95 \% \mathrm{Cl}, 0.62-1.32$, respectively]. Other parameters associated with hospitalization are shown in Tables 4.S1 and 4.S2.

A

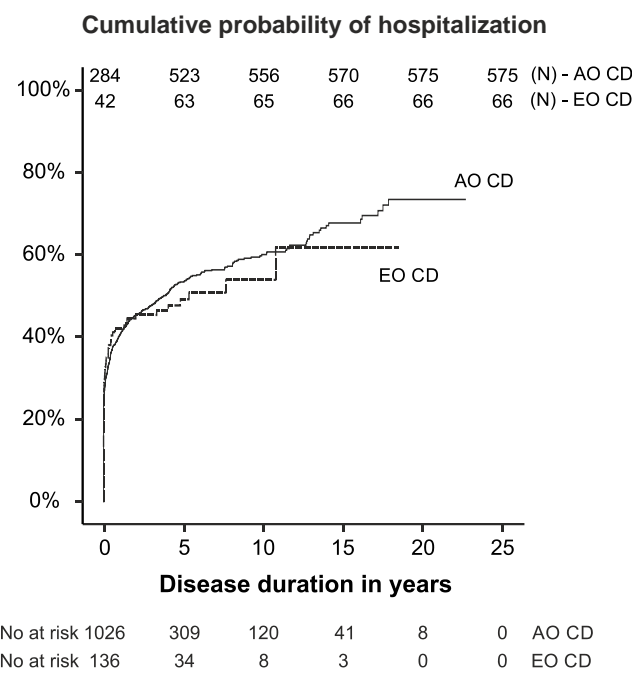

B

\section{Cumulative probability of hospitalization}

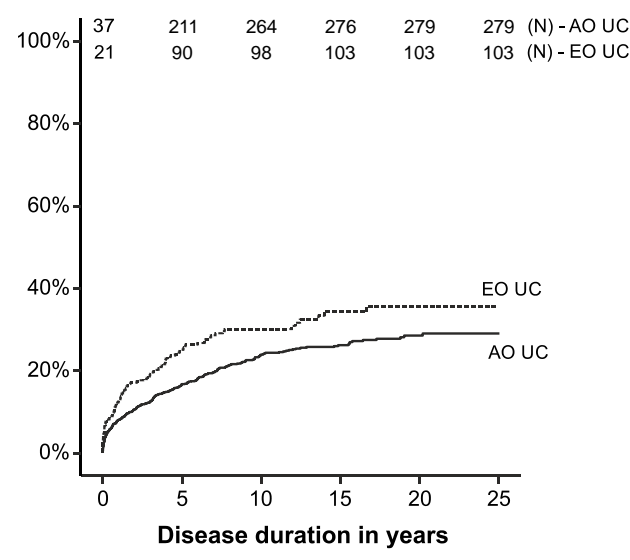

$\begin{array}{lllllll}\text { No at risk } 1288 & 743 & 426 & 218 & 29 & 0 & \text { AO UC }\end{array}$ $\begin{array}{lllllll}\text { No at risk } 373 & 173 & 91 & 43 & 6 & 0 & \text { EO UC }\end{array}$

Figure 4.4. Hospitalization rates in patients with adult-onset and elderly-onset Crohn's disease [A] and ulcerative colitis [B].

$\mathrm{N}$, number of patients who were ever hospitalized 


\section{Disease outcome: surgery}

The surgery rate is shown in Figure 4.5A [CD] and Figure 4.5B [UC]. In CD, more patients with EO than $\mathrm{AO}$ underwent surgery at diagnosis [14.1\% versus $7.1 \%$, adjusted $\mathrm{HR}, 1.88 ; 95 \% \mathrm{Cl}$, 1.12-3.15]. This discrepancy was mainly driven by a difference in surgery for inflammatory disease [11.0\% versus 4.6\%, adjusted $\mathrm{HR}, 2.28 ; 95 \% \mathrm{Cl}, 1.26-4.12]$. In $U \mathrm{C}$, only a few patients underwent surgery at diagnosis: $0.9 \%$ of the $\mathrm{AO}$ and $0.5 \%$ of the EO patients, $p=$ 0.62. In $C D$, the cumulative 5-year and 10-year surgery rates were $27.1 \%$ and $34.2 \%$ for $A O$ disease and $29.2 \%$ and $32.9 \%$ for $E O$ disease. In CD, no difference in the overall surgery rate was observed between $\mathrm{AO}$ and $\mathrm{EO}$ disease during follow-up [adjusted $\mathrm{HR}, 1.19 ; 95 \% \mathrm{Cl}$, $0.85-1.67]$. However, the surgery rate for stricturing disease was 3.5 -fold lower in patients with EO CD [adjusted HR, 0.29; $95 \% \mathrm{Cl}, 0.11-0.80$ ]. In UC, the cumulative 5- and 10-year colectomy rates were $7.0 \%$ and $8.0 \%$ in EO disease and $5.8 \%$ and $7.8 \%$ in $\mathrm{AO}$ disease, being not different between age groups [adjusted $\mathrm{HR}, 0.88 ; 95 \% \mathrm{Cl}, 0.53-1.46$ ]. Other parameters associated with surgery are shown in Tables 4.S1 and 4.S2.

A

\section{Cumulative probability of surgery}

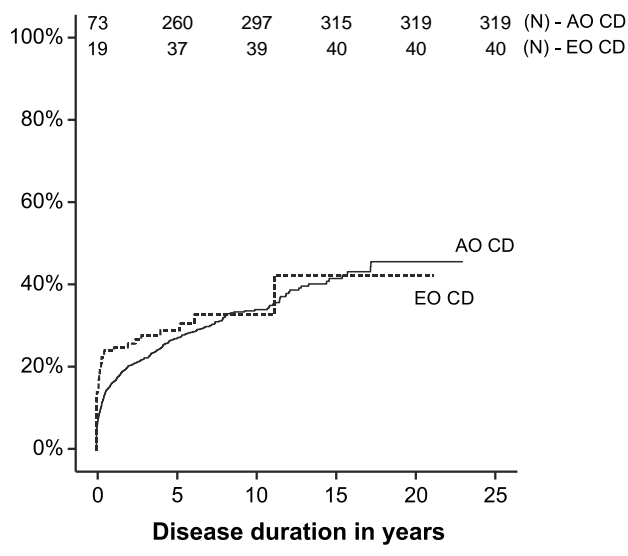

B

\section{Cumulative probability of surgery}

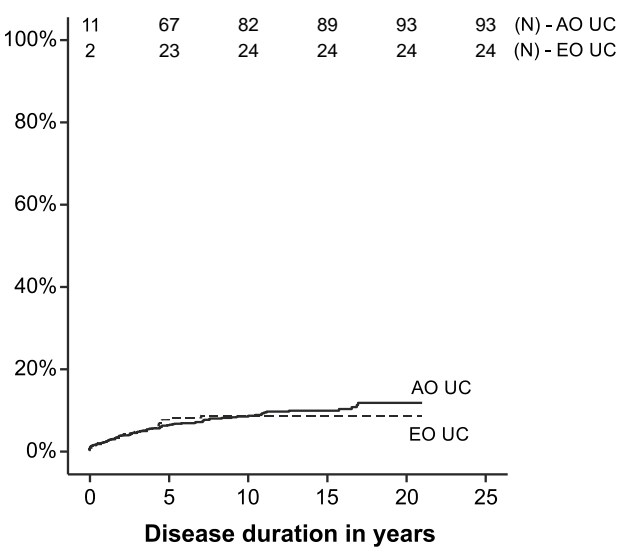

$\begin{array}{lllllll}\text { No at risk } 1288 & 859 & 531 & 286 & 36 & 0 & \text { AO UC }\end{array}$ No at risk $373 \quad 218 \quad 121 \quad 61 \quad 11 \quad 0 \quad$ EO UC

$\begin{array}{lcccccc}\text { No at risk } 136 & 46 & 9 & 4 & 1 & 0 & \text { EO CD }\end{array}$

Figure 4.5. Surgery rates in patients with adult-onset and elderly-onset Crohn's disease [A] and ulcerative colitis [B].

$\mathrm{N}$, number of patients who underwent surgery 


\section{Discussion}

Up to now, this is the largest population-based study in which the epidemiology of IBD diagnosed at elderly age was studied and in which the long-term outcome was directly compared with IBD diagnosed at adult age. This study showed that the incidence of EO IBD is rapidly increasing, along with $\mathrm{AO}$ disease. The disease presentation in $\mathrm{EO}$ patients differed from $A O$ patients in terms of disease location in both $C D$ and $U C$, hospitalization risk in UC, and surgery risk in CD. During the disease course, patients with EO UC had a higher risk of being hospitalized. Disease progression, rehospitalization risk, and surgery risk were not different between age groups in CD, nor in UC, despite the fact that patients with EO IBD were significantly less often treated with immunomodulators or anti-TNFa agents.

This study found that the increase in IBD incidence also applies to the elderly population, in particular for UC. Together with population aging, the number of elderly patients with IBD can be expected to increase even further in the upcoming years. In line with data from the European EC-IBD cohort and the Hungarian registry, we observed that CD was rare at older age [2.51 per 100,000] in the 1990s. ${ }^{11,40}$ In the South Limburg area, CD is nowadays nearly as common in the elderly as it was in adults in the 1990s [6.23 per 100,000 versus 6.84 per 100,000, respectively]. The increase in CD incidence was less profound in the elderly than in the adult population, suggesting a difference in the evolution of risk factor exposure between age groups. For instance, changes in dietary patterns were proposed to be related to the rising CD incidence. ${ }^{46,47}$ In this light, the elderly population may be less susceptible for changes in dietary patterns than younger people. For UC, the incidences in the adult and elderly population are more alike even as their evolution, indicating that risk factor exposure for UC has changed to a similar extent for both populations.

In line with previous studies on the topic, we found a different presentation of EO IBD. First, a substantial proportion of our patients with EO CD [14\%] was already operated upon diagnosis, whereas no more patients with EO than AO UC underwent surgery at diagnosis. The same observation was performed in some, ${ }^{29,41}$ but not all studies. ${ }^{40}$ This difference between CD and UC may be explained by the fact that alarming systemic complaints, such as weight loss and fever, are more frequently seen in $\mathrm{CD} .{ }^{41}$ These first symptoms may impose as an acute abdomen, necessitating an urgent surgical intervention. Second, more patients with EO UC were hospitalized at diagnosis, possibly as a result of a more severe first flare of the disease. ${ }^{48,49}$ In this study, we observed more left-sided disease and less rectal disease in the elderly population, in line with previous data. ${ }^{40,41}$ Under the assumption that UC involves the rectum first and subsequently progresses to the proximal colon, it can be hypothesized that time from disease onset to disease diagnosis may be prolonged in older patients. However, the opposite seems to be true: other studies reported that the delay time in the elderly is half 
of that of the adult population, ${ }^{34,38,41}$ pointing to a long asymptomatic prodromal phase or a different disease phenotype in the elderly patient. In line with other studies, this study found that $C D$ is not often located in both the ileum and colon. However, in contrast to most literature, ileal, and not colonic, location was the most common localization of CD in the elderly in our cohort [45.6\%]. ${ }^{29,30,36,40}$ A possible explanation for this discrepancy may reside in the high case ascertainment in the present cohort as the IBD diagnosis was confirmed by a thorough examination of the medical records, rather than by administrative diagnosis codes. Cases in which the IBD diagnosis was rejected later in the course were excluded for analyses. In this view, in particular, diverticulitis and ischemic colitis can mimic colonic CD at first presentation in the elderly. It has been found that nearly $50 \%$ of elderly patients with ischemic colitis were initially diagnosed with $\mathrm{IBD}^{50}$ and that a change in diagnosis from EO IBD to diverticulitis occurred in $8 \%$ of the cases. ${ }^{51}$ Therefore, careful exclusion of another etiology of the intestinal inflammation than IBD is warranted, especially in segmental colonic CD.

Medication use was strikingly different between $\mathrm{AO}$ and $\mathrm{EO}$ diseases in the present cohort. A 1.4-fold lower use of immunomodulators was observed in the EO CD group and a 1.5-fold lower use in the EO UC group. Based on our data, the reason for this disparity cannot be given. A milder disease course may play a role in $\mathrm{CD}$, as the use of systemic glucocorticoid therapy was also lower in the elderly group [38.7\% versus $61.0 \%]$. However, thiopurines are the preferred maintenance therapy for CD nowadays, since the efficacy of 5-ASA derivatives has been questioned. ${ }^{52}$ Therefore, the findings may implicate a lower tendency to treat elderly patients with immunosuppressive medication, possibly because of a fear of drug intolerance or immunomodulator-related infections and cancers. ${ }^{53,54}$ Potential adverse events are reported to be similar between the adult and the elderly population. ${ }^{55}$ For anti-TNF $\alpha$ agents, however, an association between elderly age and severe side infections was found. ${ }^{22,23}$ In this study, no significant difference was found in the number of adult and elderly patients discontinuing treatment because of drug-related side effects. Whether the benefit of treatment outweighs the risk of potentially enhancing infection risk and cancer development is a difficult clinical problem, especially for the elderly patient, and should be balanced for every individual patient. ${ }^{56}$

Long-term disease outcome in the elderly population has been previously assessed in some studies, yet seldom directly compared with an adult subset from the same source population. A direct comparison is mandatory to conclude that EO IBD is milder or more severe than AO IBD, as the long-term outcome may differ between IBD populations. This study found no difference in the presence of complicated disease at diagnosis between age groups or in the development thereof during follow up. An interesting observation was made in the Hungarian cohort, in which no phenotype progression was observed in the 21 patients with EO CD followed. ${ }^{40}$ The phenotype progression rate of their adult population [20\% after 5 
years] is similar to the rate observed in the adult population of this study [24\% after 5 years], indicating a more or less similar natural history of $\mathrm{CD}$ among cohorts. The explanation for the disparity in phenotype progression in EO CD may reside in the high number of elderly patients with ileal disease in the present cohort. Indeed, ileal disease was found to be a strong predictor for complicated disease..$^{57}$

Surgery is a definitive treatment option in $U C$ and has a place in the management of refractory inflammatory and complicated disease in $C D .{ }^{17,18}$ Surgical management should be balanced against medical treatment, taking into account patients' physical condition, disease activity, and therapeutic options. This balance can be complex in elderly patients, who may suffer not only from decreased renal or liver function [disfavoring medical therapy] but also from multimorbidity or an impaired physical condition [disfavoring surgical therapy]. In this study, a 5-year surgery rate of $29.2 \%$ and a 5-year colectomy rate of $7.0 \%$ were observed in patients with $E O C D$ and $E O \cup C$, respectively. These rates are in line with other populationbased studies on this topic. ${ }^{36,40,41}$ Both this study and the Hungarian study showed that IBD surgery rates in elderly are not different from the rate observed in the adult population. This conclusion is of interest, as the less frequent use of immunomodulating and anti-TNF $\alpha$ agents could have reputed otherwise. Whether a more common use of immunomodulators and antiTNF $\alpha$ agents in the elderly would lead to a lower surgery rate is an interesting question that remains to be elucidated.

Strengths of this study reside in its population-based, not claims-based, design, its large number of patients, and its direct comparison of disease presentation, medication use, and disease outcome between $\mathrm{AO}$ and EO IBD. It provides data on a clinically relevant topic regarding the disease prognosis of a group of patients with $I B D$ that is of rising importance. Some limitations have to be addressed. First, hospitalization and surgery were used as proxies for a severe disease outcome. Clinical and endoscopic disease activity scores would have provided a more reliable and detailed insight, yet are hard to collect and interpret in large, high-volume, observational cohort studies. Second, information about comorbidities, infections, and quality of life would have augmented the interpretation of the results, as they are important in the previously discussed balance between treatment benefit and risk. Quality of life is of specific interest in the elderly, in whom treatment goals may be more measured in terms of decreasing disease-specific symptoms and improving mobility and independence, rather than preventing long-term complications or surgery.

In conclusion, EO IBD is a rising entity, warranting physicians' alertness for IBD in elderly patients. The disease presentation is different, with a higher surgery risk in CD and a higher hospitalization risk in UC. Elderly patients were less often treated with immunomodulatory or biological agents, yet the long-term outcome measures were similar to AO disease, except for a higher hospitalization risk in UC. This prognostic information can be 
used in patient counseling and should be taken into account in the difficult process of selecting the most appropriate treatment strategy for the specific elderly patient. 


\section{Supplement}

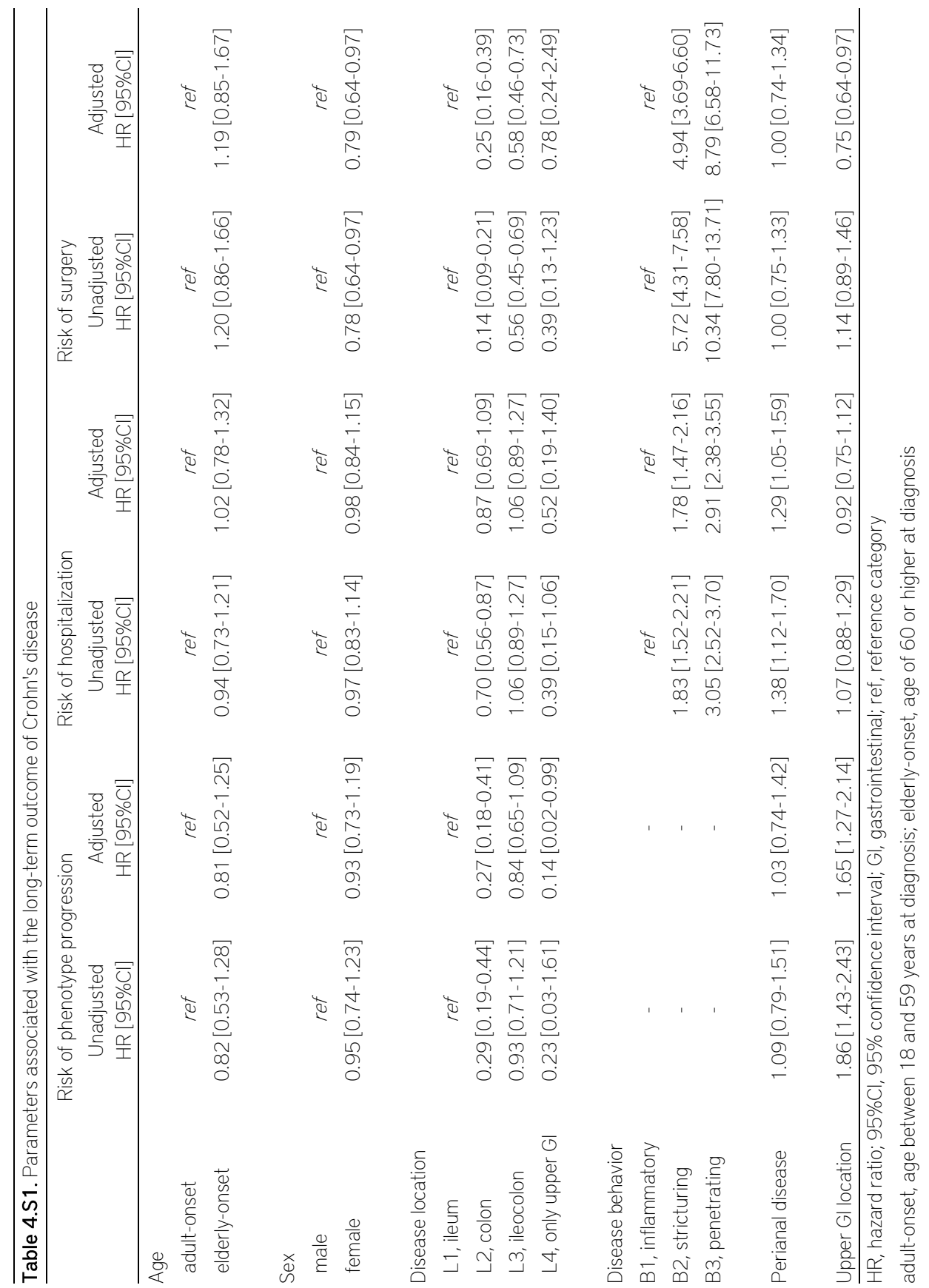




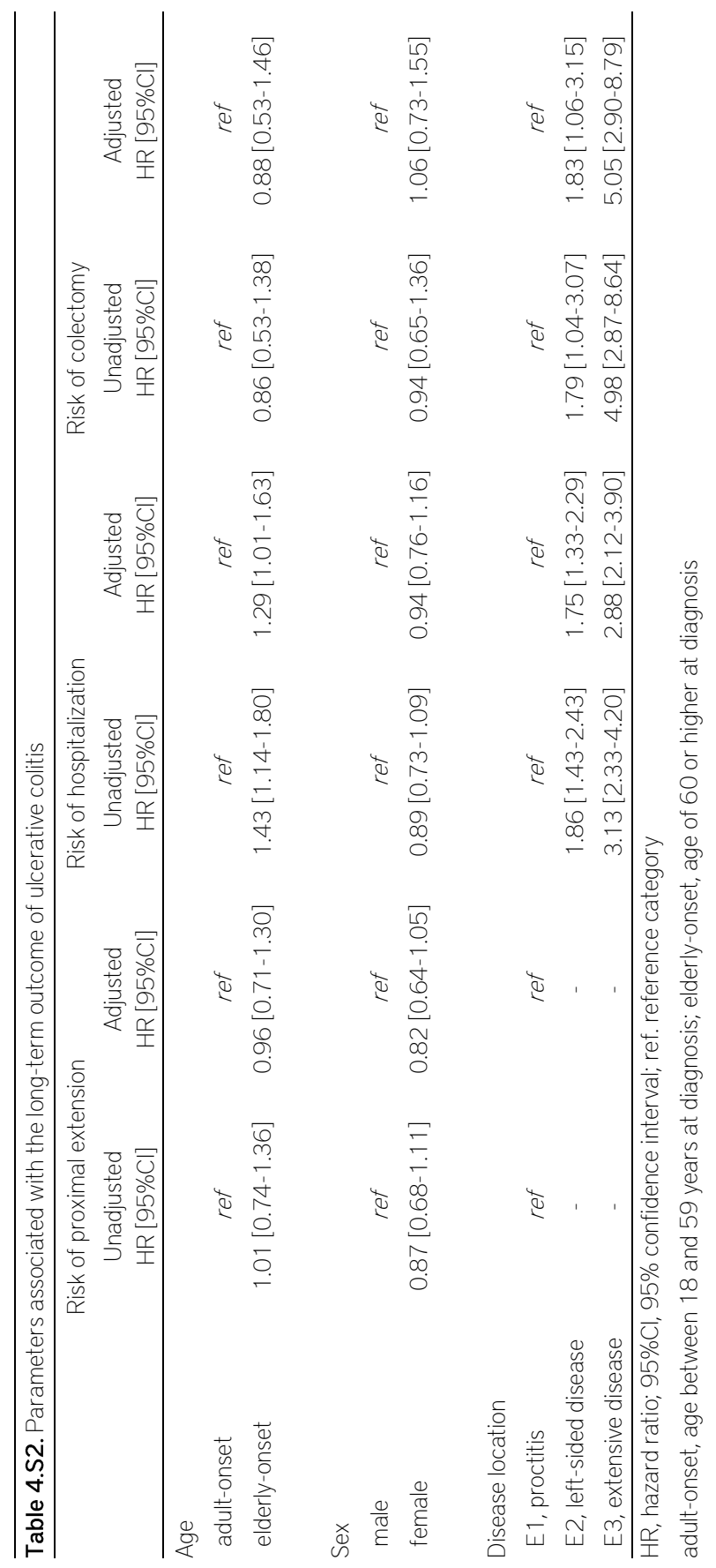




\section{References}

1. Benchimol El, Manuel DG, Guttmann A, et al. Changing age demographics of inflammatory bowel disease in Ontario, Canada: a population-based cohort study of epidemiology trends. Inflamm Bowel Dis. 2014;20:1761-1769.

2. Romberg-Camps MJ, Hesselink-van de Kruijs MA, Schouten LJ, et al. Inflammatory bowel disease in South Limburg [the Netherlands] 1991-2002: incidence, diagnostic delay, and seasonal variations in onset of symptoms. $J$ Crohns Colitis. 2009;3:115-124.

3. Vind I, Riis L, Jess $T$, et al. Increasing incidences of inflammatory bowel disease and decreasing surgery rates in Copenhagen City and County, 20032005: a population-based study from the Danish Crohn colitis database. Am J Gastroenterol. 2006;101:1274-1282.

4. Bitton A, Vutcovici M, Patenaude V, et al. Epidemiology of inflammatory bowel disease in Quebec: recent trends. Inflamm Bowel Dis. 2014;20:17701776.

5. Wei SC, Lin MH, Tung CC, et al. A nationwide population-based study of the inflammatory bowel diseases between 1998 and 2008 in Taiwan. BMC Gastroenterol. 2013;13:166.

6. Loftus CG, Loftus EV Jr, Harmsen WS, et al. Update on the incidence and prevalence of Crohn's disease and ulcerative colitis in Olmsted County, Minnesota, 1940-2000. Inflamm Bowel Dis. 2007;13:254-261.

7. Lapidus A, Bernell O, Hellers G, et al. Incidence of Crohn's disease in Stockholm County 1955-1989. Gut. 1997;41:480-486.

8. Ekbom A, Helmick C, Zack M, et al. The epidemiology of inflammatory bowel disease: a large, population-based study in Sweden. Gastroenterology.

1991;100:350-358.

9. Lucendo AJ, Hervias D, Roncero O, et al. Epidemiology and temporal trends [2000-2012] of inflammatory bowel disease in adult patients in a central region of Spain. Eur J Gastroenterol Hepatol. 2014;26:1399-1407.

10. Molodecky NA, Soon IS, Rabi DM, et al. Increasing incidence and prevalence of the inflammatory bowel diseases with time, based on systematic review. Gastroenterology. 2012;142:4654.e42; quiz e30.

11. Shivananda S, Lennard-Jones J, Logan $\mathrm{R}$, et al. Incidence of inflammatory bowel disease across Europe: is there a difference between north and south? Results of the European Collaborative Study on Inflammatory Bowel Disease [EC-IBD]. Gut. 1996;39:690-697.

12. Bernstein $\mathrm{CN}$, Blanchard JF, Rawsthorne $\mathrm{P}$, et al. Epidemiology of Crohn's disease and ulcerative colitis in a central Canadian province: a populationbased study. Am J Epidemiol. 1999; 149:916-924.

13. Herrinton LJ, Liu L, Lewis JD, et al. Incidence and prevalence of inflammatory bowel disease in a Northern California managed care organization, 1996-2002. Am J Gastroenterol. 2008;103:1998-2006.

14. United Nations. World Population Ageing 2009. New York, NY: United Nations publication; 2010.

15. StatLine. Bevolking: Kerncijfers: Centraal Bureau voor de Statistiek; 2014.

Available at: http://statline.cbs.nl/. Accessed August 1, 2015.

16. Van Duin $C$, Stoeldraijer $L$. Bevolkingsprognose 2012-2060: Langer Leven, Langer Werken. Den 
Haag/Heerlen: Centraal Bureau voor de Statistiek, 2012.

17. Dignass A, Van Assche G, Lindsay JO, et al. The second European evidence-based consensus on the diagnosis and management of Crohn's disease: current management. J Crohns Colitis. 2010;4:28-62.

18. Dignass A, Lindsay JO, Sturm A, et al. Second European evidence-based consensus on the diagnosis and management of ulcerative colitis part 2: current management. J Crohns Colitis. 2012;6:991-1030.

19. Rahier JF, Magro F, Abreu C, et al. Second European evidence-based consensus on the prevention, diagnosis and management of opportunistic infections in inflammatory bowel disease. J Crohns Colitis. 2014;8:443-468.

20. Smith MA, Irving PM, Marinaki AM, et al. Review article: malignancy on thiopurine treatment with special reference to inflammatory bowel disease. Aliment Pharmacol Ther. 2010;32:119-130.

21. Beaugerie L, Brousse N, Bouvier AM, et al. Lymphoproliferative disorders in patients receiving thiopurines for inflammatory bowel disease: a prospective observational cohort study. Lancet. 2009;374:1617-1625.

22. Lichtenstein GR, Rutgeerts P, Sandborn WJ, et al. A pooled analysis of infections, malignancy, and mortality in infliximaband immunomodulator-treated adult patients with inflammatory bowel disease. Am J Gastroenterol. 2012;107:1051-1063.

23. Cottone M, Kohn A, Daperno M, et al. Advanced age is an independent risk factor for severe infections and mortality in patients given anti-tumor necrosis factor therapy for inflammatory bowel disease. Clin Gastroenterol Hepatol. 2011:9:30-35.
24. Gavazzi G, Krause KH. Ageing and infection. Lancet Infect Dis. 2002;2: 659-666.

25. Travis S. Is IBD different in the elderly? Inflamm Bowel Dis. 2008; 14 [suppl 2]:S12-S13.

26. Kim M, Katz S, Green J. Drug management in the elderly IBD patient. Curr Treat Options Gastroenterol. 2015;13:90-104.

27. Katz S, Surawicz C, Pardi DS. Management of the elderly patients with inflammatory bowel disease: practical considerations. Inflamm Bowel Dis. 2013; 19:2257-2272.

28. Harper PC, McAuliffe TL, Beeken WL. Crohn's disease in the elderly. A statistical comparison with younger patients matched for sex and duration of disease. Arch Intern Med. 1986;146:753-755.

29. Shapiro PA, Peppercorn MA, Antoniolo DA, et al. Crohn's disease in the elderly. Am J Gastroenterol. 1981;76:132137.

30. Fabricius PJ, Gyde SN, Shouler P, et al. Crohn's disease in the elderly. Gut. 1985;26:461-465.

31. Gupta S, Saverymuttu SH, Keshavarzian A, et al. Is the pattern of inflammatory bowel disease different in the elderly? Age Ageing. 1985;14: 366-370.

32. Piront $P$, Louis $E$, Latour $P$, et al. Epidemiology of inflammatory bowel diseases in the elderly in the province of liege. Gastroenterol Clin Biol. 2002;26:157-161.

33. Brandt LJ, Boley SJ, Mitsudo S. Clinical characteristics and natural history of colitis in the elderly. Am J Gastroenterol. 1982;77:382-386.

34. Wagtmans MJ, Verspaget HW, Lamers $\mathrm{CB}$, et al. Crohn's disease in the elderly: a comparison with young adults. J Clin Gastroenterol. 1998;27:129-133. 
35. Freeman HJ. Age-dependent phenotypic clinical expression of Crohn's disease. J Clin Gastroenterol. 2005;39:774-777.

36. Heresbach D, Alexandre JL, Bretagne JF, et al. Crohn's disease in the over-60 age group: a population based study. Eur J Gastroenterol Hepatol. 2004; 16:657664.

37. Tremaine WJ, Timmons LJ, Loftus EV Jr, et al. Age at onset of inflammatory

1. bowel disease and the risk of surgery for non-neoplastic bowel disease. Aliment Pharmacol Ther. 2007;25:1435-1441.

38. Triantafillidis JK, Emmanouilidis A, Nicolakis D, et al. Crohn's disease in the elderly: clinical features and long-term outcome of 19 Greek patients. Dig Liver Dis. 2000;32:498-503.

39. Triantafillidis JK, Emmanouilidis A, Pomonis E, et al. Ulcerative colitis in the elderly: clinical patterns and outcome in 51 Greek patients. J Gastroenterol. 2001;36:312-316.

40. Lakatos PL, David G, Pandur T, et al. IBD in the elderly population: results from a population-based study in Western Hungary, 1977-2008. J Crohns Colitis. 2011;5:5-13.

41. Charpentier C, Salleron J, Savoye G, et al. Natural history of elderly-onset inflammatory bowel disease: a population-based cohort study. Gut. 2014;63:423-432.

42. van den Heuvel TR, Jonkers DM, Jeuring $\mathrm{SF}$, et al. Cohort profile: the inflammatory bowel disease South Limburg cohort [IBDSL]. Int J Epidemiol. [published online ahead of print June 4, 2015]. doi 10.1093/ije/dyv088.

43. Silverberg MS, Satsangi J, Ahmad T, et al. Toward an integrated clinical, molecular and serological classification of inflammatory bowel disease: report of a working party of the 2005 Montreal World Congress of Gastroenterology. Can J Gastroenterol. 2005;19[suppl A]:5A-36A.
44. Satsangi J, Silverberg MS, Vermeire S, et al. The Montreal classification of inflammatory bowel disease: controversies, consensus, and implications. Gut. 2006;55:749-753.

45. Gasche C, Scholmerich J, Brynskov J, et al. A simple classification of Crohn's disease: report of the working party for the world congresses of gastroenterology, Vienna 1998. Inflamm Bowel Dis. 2000;6:8-15.

46. Bernstein $\mathrm{CN}$. Why and where to look in the environment with regard to the etiology of inflammatory bowel disease. Dig Dis. 2012;30[suppl 3]:28-32.

47. Thia KT, Loftus EV Jr, Sandborn WJ, et al. An update on the epidemiology of inflammatory bowel disease in Asia. Am J Gastroenterol. 2008; 103:31673182.

48. Jones HW, Hoare AM. Does ulcerative colitis behave differently in the elderly? Age Ageing. 1988; 17:410-414.

49. Zimmerman J, Gavish D, Rachmilewitz D. Early and late onset ulcerative colitis: distinct clinical features. J Clin Gastroenterol. 1985;7:492-498.

50. Brandt L, Boley S, Goldberg L, et al. Colitis in the elderly. A reappraisal. Am J Gastroenterol. 1981;76:239-245.

51. Hadithi M, Cazemier M, Meijer GA, et al. Retrospective analysis of old age colitis in the Dutch inflammatory bowel disease population. World J Gastroenterol. 2008; 14:3183-3187.

52. Feagan BG. 5-ASA therapy for active Crohn's disease: old friends, old data, and a new conclusion. Clin Gastroenterol Hepatol. 2004;2:376-378.

53. Ariyaratnam J, Subramanian V. Association between thiopurine use and nonmelanoma skin cancers in patients with inflammatory bowel disease: a meta-analysis. Am J Gastroenterol. 2014;109:163-169.

54. Kotlyar DS, Lewis JD, Beaugerie L, et al. Risk of lymphoma in patients with 
inflammatory bowel disease treated with azathioprine and 6-mercaptopurine: a meta-analysis. Clin Gastroenterol Hepatol. 2015;13:847-858.

55. Chaparro M, Ordas I, Cabre E, et al. Safety of thiopurine therapy in inflammatory bowel disease: long-term follow-up study of 3931 patients. Inflamm Bowel Dis. 2013;19:14041410

56. Lewis JD, Schwartz JS, Lichtenstein GR. Azathioprine for maintenance of remission in Crohn's disease: benefits outweigh the risk of lymphoma. Gastroenterology. 2000;118:10181024.

57. Thia KT, Sandborn WJ, Harmsen WS, et al. Risk factors associated with progression to intestinal complications of Crohn's disease in a population-based cohort. Gastroenterology. 2010;139:1147-1155. 



\section{PART II}

Impact of advances

in IBD management:

new era, better future? 



\section{CHAPTER 5}

\section{Improvements in the long-term outcome of Crohn's disease over the past two decades and the relation to changes in medical management}

Steven FG Jeuring, Tim RA van den Heuvel, Limmie Y.L. Liu, Maurice P Zeegers, Wim H Hameeteman, Marielle JL Romberg-Camps, Liekele E Oostenbrug, Ad AM Masclee, Daisy MAE Jonkers, Marieke J Pierik

Am J Gastroenterol. 2017 Feb;112(2):325-336. 


\section{Abstract}

\section{Background}

Medical treatment options and strategies for Crohn's disease [CD] have changed over the past decades. To assess its impact, we studied the evolution of the long-term disease outcome in the Dutch Inflammatory Bowel Disease South Limburg [IBDSL] cohort.

\section{Methods}

In total, 1162 CD patients were included. Three eras were distinguished: 1991-1998 [n=316], 1999-2005 [n=387], and 2006-2011 [n=459], and patients were followed until 2014. Medication exposure and the rates of hospitalization, surgery, and phenotype progression were estimated using Kaplan-Meier survival analyses and compared between eras by multivariable Cox regression models. Second, propensity score matching was used to assess the relation between medication use and the long-term outcome.

\section{Results}

Over time, the immunomodulator exposure rate increased from 30.6\% in the era 1991-1998 to $70.8 \%$ in the era $2006-2011$ at 5 years. Similar, biological exposure increased from $3.1 \%$ [era 1991-1998] to 41.2\% [era 2006-2011]. In parallel, the hospitalization rate attenuated from $65.9 \%$ to $44.2 \%$ and the surgery rate from $42.9 \%$ to $17.4 \%$ at 5 years, respectively [both $p<0.01$ ]. Progression to a complicated phenotype has not changed over time [21.2\% in the era $1991-1998$ vs. $21.3 \%$ in the era 2006-2011, $p=0.93]$. Immunomodulator users had a similar risk of hospitalization, surgery, or phenotype progression as propensity scorematched nonusers [ $p>0.05$ for all analyses]. Similar results were found for biological users [ $p>0.05$ for all analyses].

\section{Conclusions}

Between 1991 and 2014, the hospitalization and surgery rates decreased, whereas progression to complicated disease is still common in CD. These improvements were not significantly related to the use of immunomodulators and biologicals. 


\section{Introduction}

Crohn's disease $[C D]$ is a chronic inflammatory disease of the gastrointestinal tract, characterized by periods of active inflammation, alternated with periods of remission. ${ }^{1-4}$ The prognosis of CD patients is heterogeneous and hard to predict early in the disease course. ${ }^{2,5}$ The current goal of CD treatment is to achieve and maintain steroid-free clinical remission and to prevent the development of structural bowel damage in the long term, such as fistulas and abscesses, intestinal strictures, and surgery. ${ }^{6-8}$

The long-term outcome of $C D$ is preferably studied in population-based cohorts, as these comprise an unselected patient population and therefore have good external validity. ${ }^{9-11}$ Current knowledge about the prognosis of $C D$ is mainly based on data derived from such cohorts. A change in the clinical behavior of CD [i.e. progression from an inflammatory to a stricturing or penetrating phenotype] occurs in $53-62 \%$ of the patients within 10 years after initial diagnosis. ${ }^{1,12-14}$ and surgery is required in $~ 50 \%$ of cases within 10 years. ${ }^{1,2,15,16}$ These numbers illustrate that a substantial part of the CD population has an unfavorable longterm outcome.

Most outcome data derive from studies that recruited patients several decades ago, in an era in which treatment options and strategies were different from now. Population-based studies have shown that the use of 5-aminosalicylic acid has decreased over the past two decades, whereas immunosuppressive therapy is more often prescribed. ${ }^{17-18} \mathrm{~A}$ milestone in $\mathrm{CD}$ management was the extension of the therapeutic armamentarium with anti-tumor necrosis factor- $\alpha$ [TNF $\alpha$ ] agents. In the Netherlands, infliximab was the first anti-TNF $\alpha$ agent registered in 1999 ${ }^{19-20}$ followed by the registration of adalimumab in 2007..$^{21,22}$ Randomizedcontrolled trials and meta-analyses have shown the efficacy of immunomodulators and antiTNF $\alpha$ agents in inducing and maintaining disease remission. ${ }^{23-26}$ Whether the adoption of these treatments resulted in an improved long-term outcome of CD in general is important information for clinicians and patients, as well as for health-care planning.

The primary aim of this study was to evaluate changes in the medical management and long-term outcome of CD between 1991 and 2014 in a large, well-characterized Dutch population-based cohort of CD patients. The secondary aim was to assess the relation between the use of immunomodulators and anti-TNF $\alpha$ agents and the long-term disease outcome. 


\section{Methods}

\section{Study population and design}

The inflammatory bowel disease South Limburg [IBDSL] cohort comprises adult IBD patients who were diagnosed between 1991 and 2011 in the South Limburg area of the Netherlands. The South Limburg area is a well-defined geographic region in the southeast of the Netherlands, bordered by Belgium and Germany and is narrowly connected to the rest of the Netherlands in the north. The relative geographic isolation results in a low net migration rate of 2.1 per 1000 inhabitants per year ${ }^{27}$, favoring population-based research. A recent completeness check showed that over 93\% of all eligible IBD patients in the South Limburg area is currently registered in the IBDSL cohort. For detailed information on the IBDSL cohort, we refer to the cohort profile. ${ }^{28}$

The present study included all 1162 CD patients registered in the IBDSL cohort. Patients were followed from the date of diagnosis to the end of data collection [2014], date of emigration, or date of death.

The primary aims of the study were to assess changes in the medical management and long-term outcome of CD between 1991 and 2014. Disease outcome was studied in terms of [I] disease progression from an inflammatory phenotype to either a stricturing or penetrating phenotype [i.e., phenotype progression or change in disease behavior], [II] hospitalization, [III] surgery and [IV] cumulative corticosteroid [CS] use. The latter was assessed as the total number of days and the total dosage of CS use within the first year and first 5 years of the disease course. As a secondary aim, the relation between the use of immunomodulators and anti-TNF $\alpha$ agents and the long-term disease outcome was assessed.

To study the evolution of the aforementioned end points over time, three time cohorts were distinguished, based on the year of CD diagnosis: era '91-'98 [CD diagnosis between 1991 and 1998], era '99-'05 [CD diagnosis between 1999 and 2005], and era '06-'11 [CD diagnosis between 2006 and 2011]. Era '91-'98 can be referred to as the "pre-biological era", as the first biological agent [infliximab] was registered in 1999 for CD in the Netherlands. The biological era was further equally divided in two subsequent cohorts [eras '99-'05 and '06-'11].

This study was approved by the Medical Ethics Committee of the Maastricht University Medical Center [NL31636.068.10] and the IBDSL cohort is registered in ClinicalTrial.gov [NCT02130349]. 


\section{Data collection and definitions}

Demographic data, as well as clinical data on the occurrence of phenotype progression, hospitalization, and surgery, were collected from medical records by scrutinizing patient files using a standardized case report form. Phenotype progression was defined as the development of either intestinal strictures [B2] or internal fistulas or abscesses [B3] in patients with an inflammatory phenotype [B1], according to the Montreal consensus. ${ }^{29}$ To facilitate data comparison with older studies, disease behavior was also classified according to the Vienna consensus. ${ }^{30}$ Hospitalization was defined as a hospital admission for CD-related complaints, CD-related surgery, or both. Elective admissions for endoscopy procedures or regular drug administration were excluded. Surgery was defined as the resection of a part of the bowel because of intestinal inflammation or a CD-related complication [such as a stenosis, fistula or perforation]. Perianal surgeries were not included in the analyses.

Corticosteroid treatment was defined as the use of a systemic CS, such as prednisone. CSs that have a local effect, such as budesonide, were not considered in this study. Dates of treatment initiation, cessation, and dose adjustments were retrieved from the medical records. In case no comment on the tapering regimen was specified, and CSs were not used by the patient at the next visit, a standard tapering regimen of $5 \mathrm{mg}$ per week was considered as from the last visit in which CS use was mentioned. The total cumulative dosage and the total number of days were determined including all CS prescriptions within 1 and 5 years after diagnosis. These analyses were performed only in patients having such a long follow-up to avoid extrapolation of the data. Extrapolation could lead to an overestimation of the total CS use in more recently diagnosed patients, as follow-up is shortest in this group and the probability of requiring CS courses attenuates during disease course. ${ }^{31}$

Immunomodulators comprised azathioprine, mercaptopurine, tioguanine and methotrexate, and anti-TNF $\alpha$ agents considered were both agents registered in the Netherlands for CD [infliximab and adalimumab]. Dates of treatment initiation and cessation were retrieved from the medical records.

\section{Statistical analyses}

Continuous data were presented as means with standard deviations in case of parametric data and as medians with interquartile range [IQR] in case of nonparametric data. Data were subsequently compared between eras by one-way analysis of variance or Kruskal-Wallis test, respectively. Dichotomous and nominal data were presented as absolute numbers and percentages and compared by $\chi^{2}$ tests.

The cumulative probability of event occurrence [phenotype progression, hospitalization, and surgery], as well as the cumulative probability of exposure to immunomodulators and anti-TNF $\alpha$ agents, was estimated by Kaplan-Meier survival statistics 
to adjust for differences in follow-up between patients. Differences in cumulative probabilities between groups were determined by using the log-rank test. In addition, a multivariable Cox regression analysis was used to model the association between the era of diagnosis and the event of interest, adjusting for known clinical confounders. Age at diagnosis, sex, disease location at diagnosis, disease behavior at diagnosis and the era of diagnosis were included in all multivariable models. Differences in hazards between eras were expressed as hazard ratios [HRs] with 95\% confidence intervals [95\% Cls]. The proportional hazards assumption was tested for all variables by adding a time-dependent interaction term to the specific model. Time dependency was observed for the outcomes hospitalization and surgery. Therefore, two models were created: one for estimating the outcome risk at diagnosis [defined as within 1 month after diagnosis] and a second model for estimating the outcome risk during follow-up [i.e., beyond 1 month after diagnosis]. This approach was used because one model would lead to an underestimation of the early and an overestimation of the late risk $k^{32}$ and because a difference in outcome risk between diagnosis and follow-up is clinically relevant.

As treatment strategies have changed over time, we subsequently studied the relation between the use of immunomodulators and anti-TNF $\alpha$ agents and the long-term disease outcome. First, we designed propensity score models in which medication users were matched to nonusers. Second, patients were followed as from the date therapy was started [users] or a corresponding matched index date [nonusers]. By using this approach, confounding by indication ${ }^{33}$ and immune-time bias ${ }^{34}$ were minimized, respectively. Separate models were created for immunomodulators and anti-TNFa agents for each outcome [hospitalization risk, phenotype progression risk and surgery risk]. We defined "users" as patients with a first immunomodulator or anti-TNFa prescription within 2 years after CD diagnosis. The period of 2 years was chosen because the temporal changes in the long-term outcome were found to occur within this time span. Users and nonusers were required to have a B1 phenotype at the index date for the analyses on phenotype progression and to be surgery naive for the analyses on surgery risk. First, a propensity score was generated for every patient, using binary logistic regression with start immunomodulator or anti-TNF $\alpha$ therapy within 2 years as dependent variable. Parameters known or suspected to be associated with receiving the specific therapy as well as with the risk of the specific outcome were included, i.e., age at diagnosis, sex, and early course characteristics [i.e., disease location, behavior, hospitalization, CS use, and immunomodulator use [anti-TNFa models only] within 3 months after diagnosis]. Subsequently, medication users were matched to nonusers based on the propensity score using a 1:1 neighborhood matching method and a caliper of 0.20 . The first 3 months after the index date were censored, as this period is necessary to reach full therapy efficacy and events within this time window are likely the result of reverse causation. Followup ended when the event of interest occurred, therapy was stopped [including six months of 
lag time], or the end of data collection was reached. The cumulative probability of event occurrence was studied by using Kaplan-Meier survival analyses and Cox regression analyses. Results were adjusted for differences in cumulative days of CS use before the index date between users and nonusers by including this parameter in the Cox regression models. In order to evaluate the robustness of the model, sensitivity analyses were conducted in which cutoff values of 6 and 12 months after CD diagnosis were used for the definition of "user". Results of these analyses are presented in Supplementary Table 5.S1.

Two-sided $p$-values of 0.05 were considered statistically significant. All statistical analyses were conducted using IBM SPSS Statistics for Windows, Version 23.0. [IBM, Armonk, NY]. 


\section{Results}

\section{Patient population}

In total, 1162 CD patients were included in this study. Era '91-'98 comprised 316 patients, era '99-'05 comprised 387 patients, and era '06-'11 comprised 459 patients. These patients were followed for a median of 16.1 [IQR 10.3-19.3], 9.7 [IQR 8.0-11.8], and 4.2 [IQR 2.9-5.6] years, respectively. Patient characteristics are presented in Table 5.1.

\begin{tabular}{|c|c|c|c|c|c|}
\hline & & $\begin{array}{l}\text { era } 1991-1998 \\
\quad[N=316]\end{array}$ & $\begin{array}{l}\text { era 1999-2005 } \\
\quad[N=387]\end{array}$ & $\begin{array}{c}\text { era 2006-2011 } \\
{[N=459]}\end{array}$ & \\
\hline Age in years ${ }^{a}$ & mean [SD] & $36.2[15.8]$ & $37.5[15.8]$ & $38.8[16.1]$ & $p=0.08$ \\
\hline Male & $N[\%]$ & 121 [38.3] & $137[35.4]$ & 176 [38.3] & $p=0.63$ \\
\hline Current smoker ${ }^{a, b}$ & N [\%] & $141[55.3]$ & $178[49.4]$ & $175[46.3]$ & $p=0.09$ \\
\hline $\begin{array}{l}\text { Disease duration } \\
\text { in years }\end{array}$ & $\begin{array}{l}\text { median } \\
{[\text { IQR }]}\end{array}$ & 16.1 [10.3-19.3] & $9.7[8.0-11.8]$ & $4.2[2.9-5.6]$ & $p<0.01$ \\
\hline Disease location & & & & & $p=0.04$ \\
\hline L1, ileum & $N[\%]$ & 155 [49.1] & 170 [43.9] & 175 [38.1] & \\
\hline L2, colon & $N[\%]$ & 94 [29.7] & 127 [32.8] & 150 [32.7] & \\
\hline L3, ileocolon & $N[\%]$ & 63 [19.9] & $80[20.7]$ & $124[27.0]$ & \\
\hline L4, only upper Gl & $N[\%]$ & $4[1.3]$ & 10 [2.6] & 10 [2.2] & \\
\hline Perianal disease ${ }^{a}$ & $N[\%]$ & 30 [9.5] & $27[7.0]$ & 37 [8.1] & $p=0.48$ \\
\hline Upper Gl location $^{a}$ & $N[\%]$ & $21[6.6]$ & $43[11.1]$ & $60[13.1]$ & $p=0.02$ \\
\hline
\end{tabular}

\section{Medical therapy}

Immunomodulator treatment was ever used by 704 CD patients [60.6\%]. The cumulative 5year exposure rate increased from 30.6\% [era '91-'98] to 56.7\% [era '99-'05] to $70.8 \%$ [era '06-'11] [ $p<0.01$ ], see Figure 5.1A. In line, the time to the first prescription decreased, reflected by an increasing number of patients who commenced treatment within the first year of disease: 9.1\% [era '91-'98], 31.0\% [era '99-'05], and 49.5\% [era '06-'11] $[p<0.01]$.

Anti-TNFa therapy was ever used by 379 CD patients [32.6\%]. It became available during the course of patients diagnosed in the era '91-'98, whereas it was available as from diagnosis in patients diagnosed in the eras '99-'05 and '06-'11. The cumulative 5-year exposure to anti-TNF $\alpha$ treatment increased from 3.1\% [era '91-'98] to 19.9\% [era '99- 
A

\section{Cumulative exposure to immunomodulators}

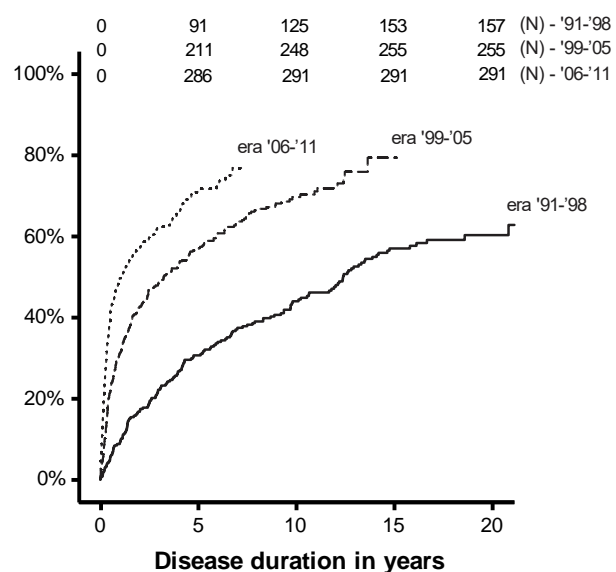

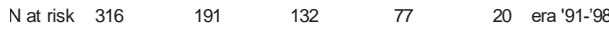

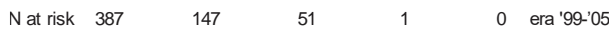

B

\section{Cumulative exposure to anti-TNF $\alpha$ agents}

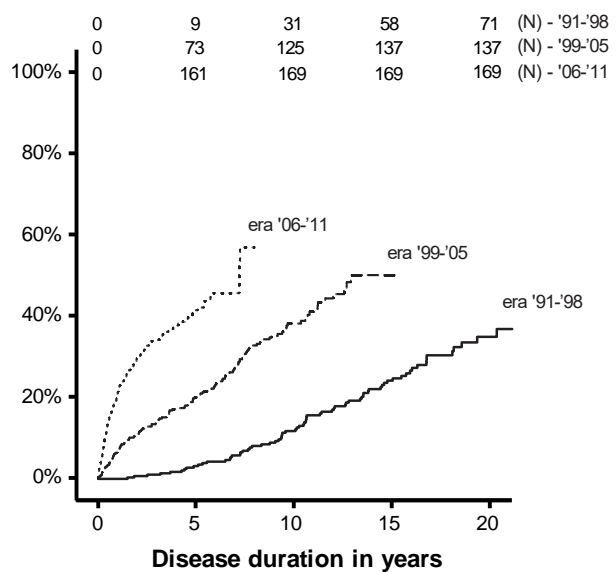

$\begin{array}{lcccccc}\mathrm{N} \text { at risk } & 316 & 268 & 210 & 139 & 37 & \text { era '91-'98 } \\ \mathrm{N} \text { at risk } & 387 & 272 & 107 & 1 & 0 & \text { era '99-'05 } \\ \mathrm{N} \text { at risk } & 459 & 92 & 0 & 0 & 0 & \text { era '06-'11 }\end{array}$

Figure 5.1. The cumulative exposures to immunomodulators [A] and anti-TNF $\alpha$ agents [B] among the three eras. $N$, number of patients who ever used the specific therapy. Era '91-'98, patients diagnosed between 1991 and 1998; era '99-'05, patients diagnosed between 1999 and 2005; era '06-'11, patients diagnosed between 2006 and 2011.

'05] to 41.2\% [era '06-'11] [ $p<0.01$ ], see Figure 5.1B. As with immunomodulators, a decrease in the time to first exposure was observed, reflected by an increase in the number of patients using anti-TNF $\alpha$ therapy within the first year after diagnosis: 0\% [era '91-'98], 7.1\% [era '99-'05], and 21.2\% [era '06-'11] [ $p<0.01$ ]. Even within the biological era [eras '99-'05 and era '06-'11], a 3.2-fold increase in the early use of anti-TNF $\alpha$ therapy [i.e., within 1 year] was observed [adjusted HR [aHR] 3.24; 95\% Cl 2.10-4.99]. Many patients exposed to biological therapy were on combination therapy [anti-TNF $\alpha+$ immunomodulator] at the start of the anti-TNF $\alpha$ agent [43.5\%] and in 10.8\% an immunomodulator was [re]introduced during biological therapy. In 46.1\%, the immunomodulator was withdrawn during follow-up.

\section{Disease behavior}

In total, 460 patients [39.6\%] had a complicated CD behavior, of whom 205 [17.7\%] already at diagnosis [Figure 5.2A]. 
A

\section{Distribution of disease behavior over course}

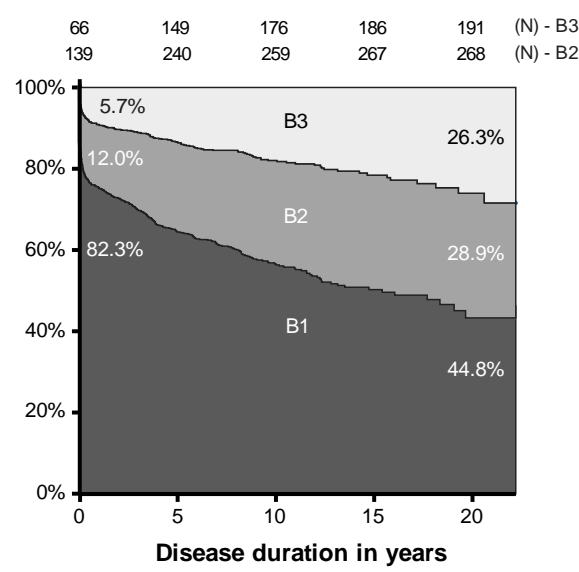

B

Progression to a complicated disease behavior

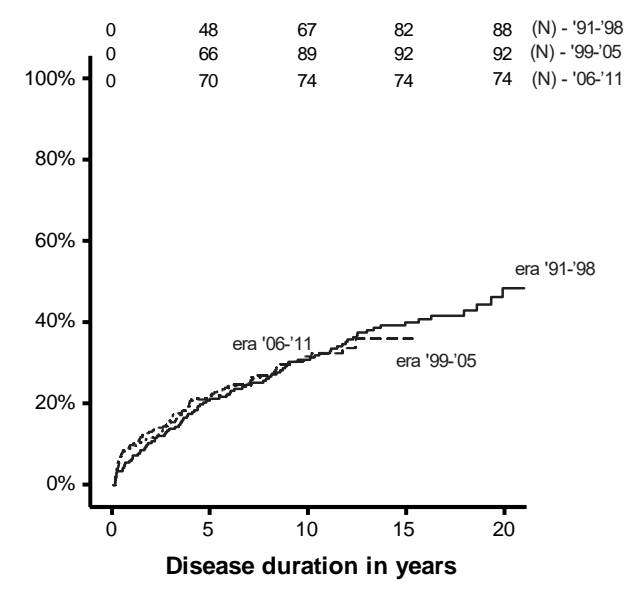

$\begin{array}{lrrrrrr}\mathrm{N} \text { at risk } & 240 & 167 & 131 & 86 & 25 & \text { era '91-'98 } \\ \mathrm{N} \text { at risk } & 319 & 106 & 96 & 1 & 0 & \text { era '99-'05 } \\ \mathrm{N} \text { at risk } & 396 & 41 & 0 & 0 & 0 & \text { era '06-'11 }\end{array}$

Figure 5.2. Disease behavior, classified according to the Montreal consensus. [A] the distribution of disease behavior over disease course in the full IBDSL cohort. [B] the cumulative probability of progression from a B1 to either a B2 or B3 phenotype in patients with B1 disease at diagnosis, stratified by era of diagnosis.

$\mathrm{N}$, number of patients who have the specific phenotype or showed progression. B1, nonstricturing, nonpenetrating disease; B2, stricturing disease; B3, penetrating disease. Era '91-'98, patients diagnosed between 1991 and 1998; era '99-'05, patients diagnosed between 1999 and 2005; era '06-'11, patients diagnosed between 2006 and 2011.

Over time, the number of patients with complicated disease at diagnosis decreased from 24.1\% [era '91-'98] to 17.1\% [era '99-'05] to 13.8\% [era '06-'11] [ $p<0.01$ ]. The

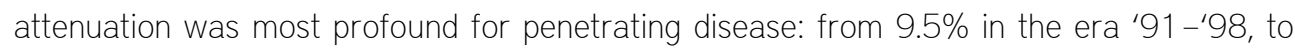
$4.5 \%$ in the era '99-'05, and to $3.7 \%$ in the era '06-'11 [ $p<0.01]$. The cumulative 5-year risk of having a complicated behavior was $40.2 \%$ in the era ' $91-' 98,35.1 \%$ in the era '99'05, and 32.1\% in the era '06-'11 [ $p=0.06]$. Progression from B1 to either B2/B3 phenotype during disease course was observed in 255 patients, corresponding to a cumulative 5-year probability rate of $21.6 \%$. Over time, no change in the progression rate was observed: 21.2\% [era '91-'98] vs. 21.7\% [era '99-'05] vs. 21.3\% [era '06-'11] $[p=0.93]$, see Figure 5.2B. Phenotype progression was found to be associated with an ileal and upper gastrointestinal location of the disease at diagnosis [Tables 5.2 and 5.3]. 


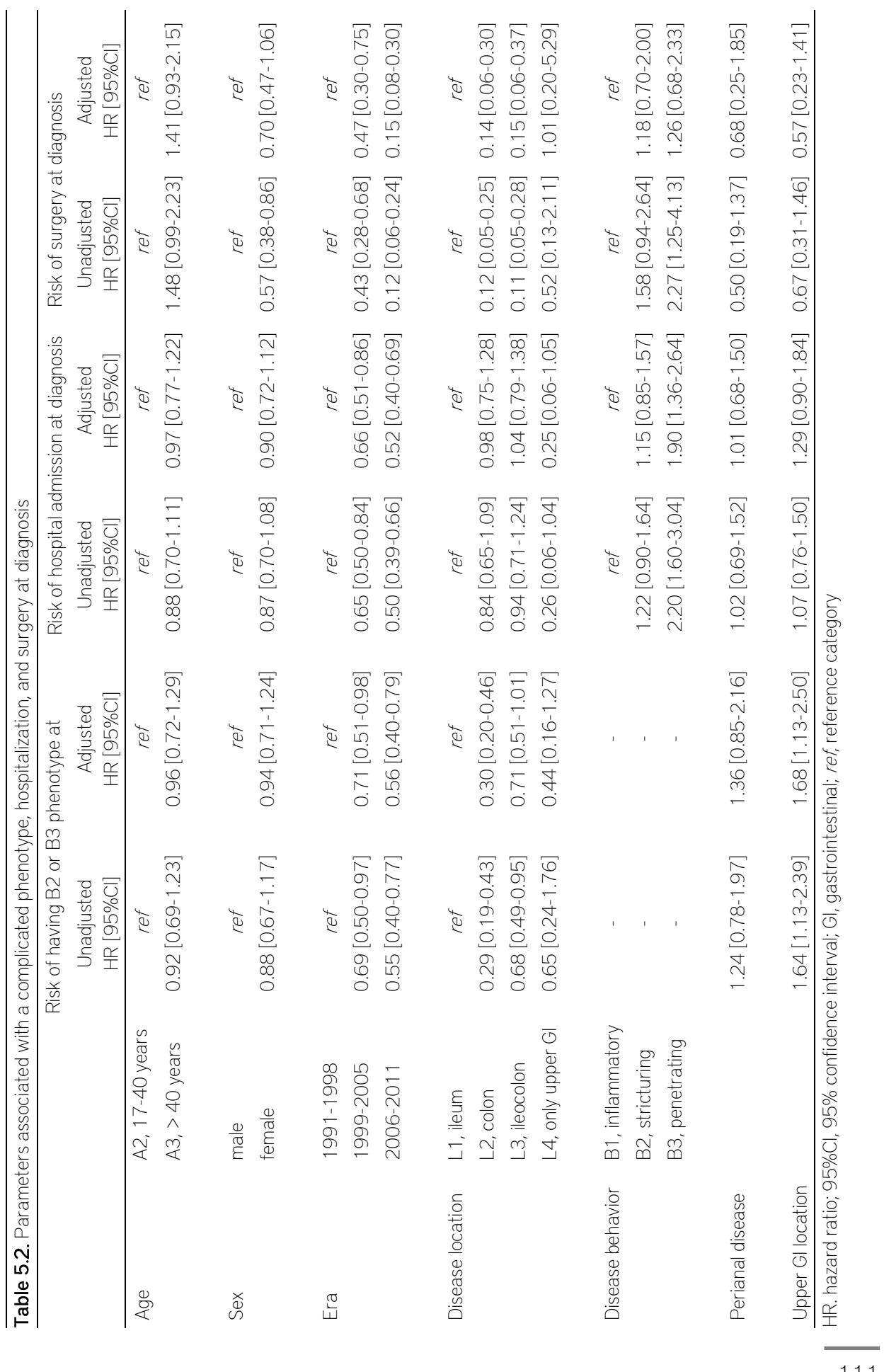




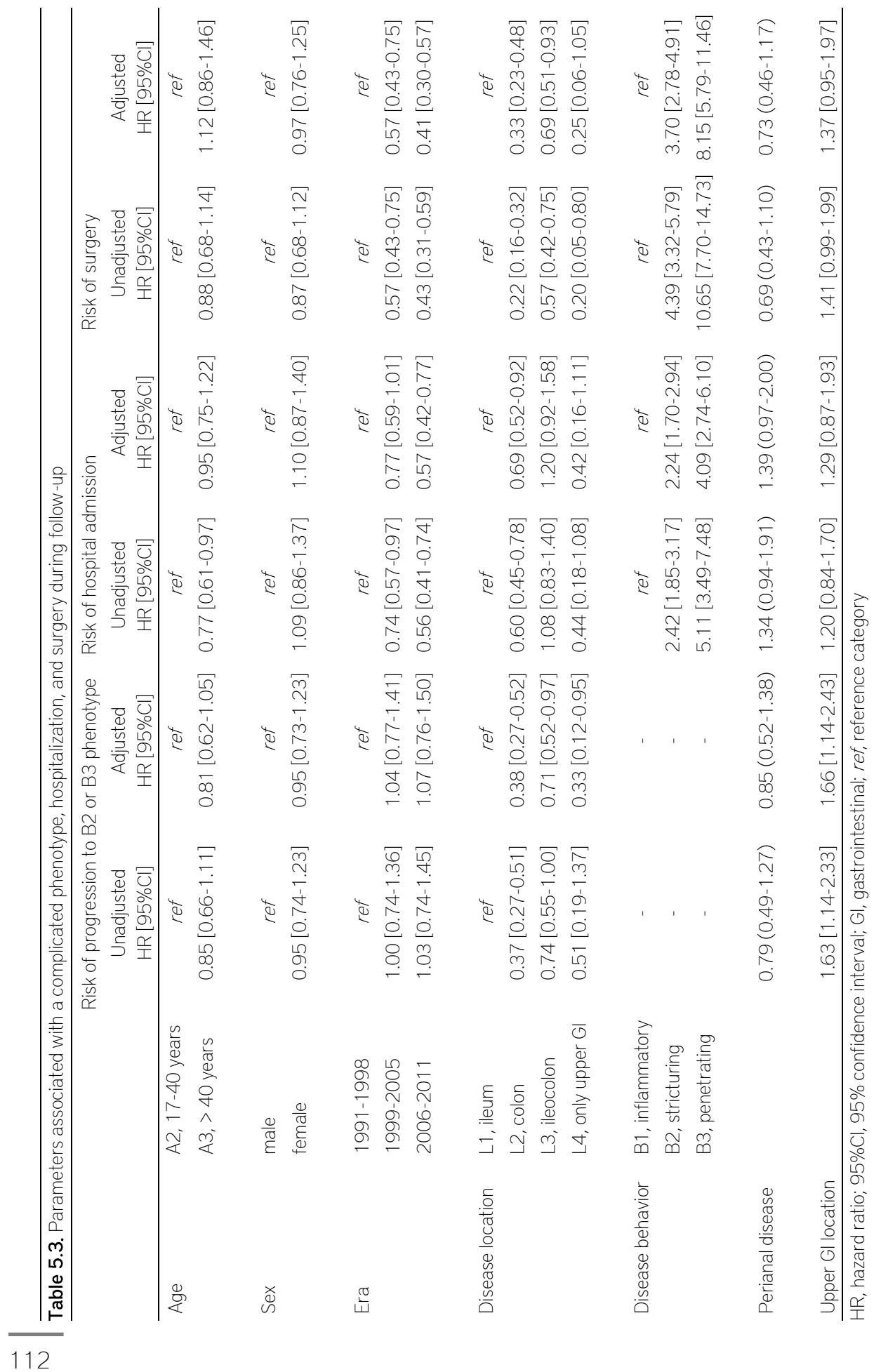


In the propensity score model for phenotype progression, 264 immunomodulator users could be matched to 264 nonusers. The cumulative 5-year probability of phenotype progression was $19.0 \%$ in users vs. $22.8 \%$ in nonusers that did not differ significantly between groups [aHR 1.01; 95\% Cl 0.60-1.71]. For the anti-TNF $\alpha$ analyses, 110 anti-TNF $\alpha$ users could be matched to 110 nonusers, also resulting in a similar cumulative 5-year probability of phenotype progression between groups [21.3\% vs. 21.0\%, aHR 0.76; 95\% Cl 0.39-1.48]. Results were similar in the sensitivity analyses, with cutoff values of 6 and 12 months for medication use [Supplementary Table 5.S1].

\section{Hospitalization}

In total, 1221 CD-related hospitalizations took place in 644 patients. Overall, the cumulative 5-year probability of hospitalization attenuated over time, from 65.9\% [era '91-'98] to 53.1\% [era '99-'05] and to 44.2\% [era '06-'11] [ $p<0.01]$, see Figure 5.3A.

A

Cumulative risk of hospitalization

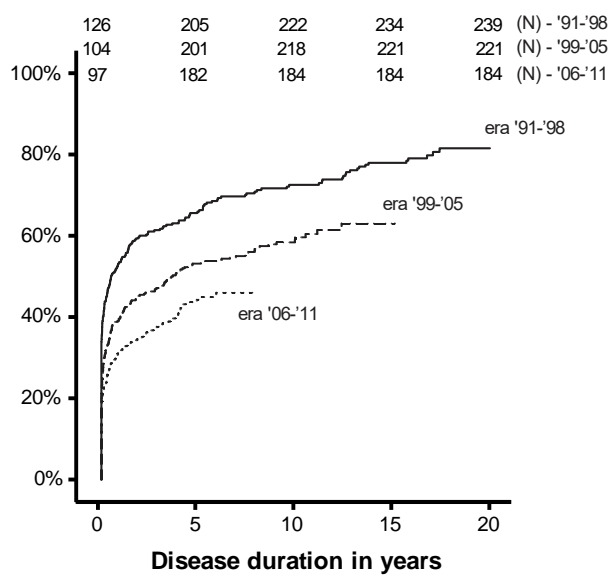

$\begin{array}{lcccccc}\mathrm{N} \text { at risk } & 316 & 93 & 64 & 44 & 8 & \text { era '91-'98 } \\ \mathrm{N} \text { at risk } & 387 & 159 & 64 & 1 & 0 & \text { era '99-'05 } \\ \mathrm{N} \text { at risk } & 459 & 90 & 0 & 0 & 0 & \text { era '06-'11 }\end{array}$
B

Cumulative risk of a surgical resection

\begin{tabular}{|c|c|c|c|c|}
\hline 54 & 133 & 153 & 169 & 173 \\
\hline 29 & 98 & 114 & 117 & 117 \\
\hline $00 \%-10$ & 67 & 70 & 70 & 70 \\
\hline
\end{tabular}

Figure 5.3. The cumulative risks of hospitalization $[\mathrm{A}]$ and surgery $[\mathrm{B}]$ among the three eras.

$\mathrm{N}$, number of patients who had the specific event. Era '91-'98, patients diagnosed between 1991 and 1998; era '99-'05, patients diagnosed between 1999 and 2005; era '06-'11, patients diagnosed between 2006 and 2011.

Part of this effect was found to be explained by a decrease in the hospitalization rate at diagnosis: 39.9\% in the era '91-'98, 27.0\% in the era '99-'05, and 21.2\% in the era '06$' 11[p<0.01]$ [Table 5.2]. However, if we only consider patients not hospitalized at diagnosis, the decrease in hospitalization risk remains [Table 5.3]. Patients with a complicated phenotype 
at diagnosis had a higher risk of hospitalization during follow-up. The median number of days admitted per hospitalization decreased from 14.5 days [IQR 10.0-27.0] in the era '91-'98 to 11.0 days [IQR 7.0-18.0] in the era '99-'05 to 8.0 days [IQR 5.0-14.0] in the era '06-'11 $[p<0.01]$. No difference was found in the probability of a rehospitalization within the first 5 years after diagnosis: 34.0\% [era '91-'98] vs. 31.5\% [era '99-'05] vs. 38.4\% [era '06-'11] $[p=0.29]$.

In the propensity score model for hospitalization, 352 immunomodulator users could be matched to 352 nonusers. The cumulative 5-year probability of hospitalization was higher in users than in nonusers [35.6\% vs. 27.0\%], but did not reach statistical significance [aHR 1.25; 95\% Cl 0.86-1.80]. For anti-TNFa therapy, 168 users could be matched to 168 nonusers and the cumulative 5-year probability of hospitalization was $35.1 \%$ in users and $25.1 \%$ in nonusers that also did not reach statistical significance [aHR 1.13; 95\% Cl 0.681.87]. The sensitivity analyses showed similar results [Supplementary Table 5.S1].

\section{Surgery}

In total, 361 patients required a total of 456 surgical resections. Surgery type and indication are shown in Table 5.4.

\begin{tabular}{|c|c|c|c|c|c|}
\hline & & $\begin{array}{c}\text { era 1991-1998 } \\
\quad[N=173]\end{array}$ & $\begin{array}{c}\text { era } 1999-2005 \\
\quad[N=118]\end{array}$ & $\begin{array}{c}\text { era 2006-2011 } \\
{[\mathrm{N}=70]}\end{array}$ & \\
\hline $\begin{array}{l}\text { Indication of surgery } \\
\text { Active, luminal disease } \\
\text { Stricturing disease } \\
\text { Penetrating disease } \\
\text { Other }\end{array}$ & $\begin{array}{l}N[\%] \\
N[\%] \\
N[\%] \\
N[\%]\end{array}$ & $\begin{array}{c}79[45.7] \\
59[34.1] \\
27[15.6] \\
8[4.6]\end{array}$ & $\begin{array}{c}41[35.0] \\
44[37.6] \\
30[25.6] \\
3[2.5]\end{array}$ & $\begin{array}{c}24[34.3] \\
36[51.4] \\
8[11.4] \\
2[2.9]\end{array}$ & $p=0.03$ \\
\hline $\begin{array}{l}\text { Type of surgery } \\
\text { lleocecal resection } \\
\text { Colonic resection } \\
\text { Right hemicolectomy } \\
\text { Small intestine resection }\end{array}$ & $\begin{array}{l}N[\%] \\
N[\%] \\
N[\%] \\
N[\%]\end{array}$ & $\begin{array}{c}135[78.0] \\
19[11.0] \\
15[8.7] \\
4[2.3]\end{array}$ & $\begin{array}{c}100[84.7] \\
4[3.4] \\
9[7.6] \\
5[4.2]\end{array}$ & $\begin{array}{c}54[77.1] \\
7[10.0] \\
5[7.1] \\
4[5.7]\end{array}$ & $p=0.27$ \\
\hline
\end{tabular}

Overall, the cumulative 5-year probability of surgery gradually decreased over time, from $42.9 \%$ in the era ' $91-^{\prime} 98$ to $26.2 \%$ in the era ' $99-^{\prime} 05$, to $17.4 \%$ in the era '06-' 11 $[p<0.01$ ], see Figure 5.3B. Over time, surgeries were less often performed for active, luminal disease, and proportionally more often for stricturing disease $[p=0.03]$. Eighty surgeries were already performed at diagnosis, corresponding to a cumulative probability of $8.0 \%$. Over the past two decades, the probability of undergoing surgery at diagnosis decreased from $17.1 \%$ in the era ' 91 -'98 to $7.6 \%$ in the era ' $99-$ ' 05 to $2.2 \%$ in the era ' 06 -' 11 [ $p<0.01$ ]. A majority of these surgical resections was performed for inflammatory disease [76.3\%], either before 
or shortly after the diagnosis was established, whereas $17.5 \%$ was operated for stricturing disease and $6.3 \%$ for penetrating disease at that time. No difference herein was observed between eras [ $p=0.65]$. Results regarding the decreasing surgery risk were concordant when only patients were considered who were not operated at diagnosis: the cumulative 5 -year probability in these patients was $33.2 \%$ in the era ' 91 -' $^{\prime} 98,20.8 \%$ in the era '99-'05, and $15.9 \%$ in the era ' $06-^{\prime} 11[p<0.01]$. Besides era of diagnosis, an ileal disease location was significantly associated with an increased risk of surgery at both diagnosis and during followup, and a complicated phenotype at diagnosis was found to be associated with surgery during follow-up [Tables 5.2 and 5.3].

In the propensity score model for surgery, 315 immunomodulator users could be matched to 315 nonusers and the cumulative 5-year probability of surgery was similar between groups [19.2\% vs. $22.8 \%$, aHR $0.72 ; 95 \% \mathrm{Cl} 0.45-1.16$, respectively]. In the antiTNFa analyses, 159 users could be matched to 159 nonusers and the cumulative 5-year probability of surgery was also found to be similar between groups [24.3\% vs. $21.9 \%$, aHR $1.07 ; 95 \% \mathrm{Cl} 0.56-2.04]$. Results were concordant in the sensitivity analyses [Supplementary Table 5.S1].

\section{Cumulative corticosteroid use}

Corticosteroid therapy was ever prescribed to 665 patients [57.2\%]. The cumulative 5 -year exposure was $50.0 \%$ in the era ' 91 -' $^{\prime} 8,56.2 \%$ in the era ' $99-{ }^{\prime} 05$, and $54.8 \%$ in the era '06-'11 [ $p=0.20]$. Although the 5 -year exposure rate was similar among eras, more patients in the era '06-'11 [48.5\%] than in the era '91-'98 [38.5\%] had already used CS therapy within the first year of disease [aHR $1.32 ; 95 \% \mathrm{Cl} 1.05-1.65$ ]. Colonic and ileocolonic location of the disease at diagnosis were associated with early CS use [aHR 2.73; 95\% Cl 2.18-3.41 and aHR 2.16; 95\% Cl 1.71-2.73 as compared with ileal location, respectively]. Furthermore, an age at diagnosis of $>40$ years was associated with a lower need for CS therapy within the first year [aHR 0.69; 95\% Cl 0.57-0.83].

The cumulative CS use was determined in CD patients who had a first prescription within 5 years after diagnosis and had a follow-up of at least 5 years [ $n=430$ ] [Table 5.5]. Both the total number of days and the total dosage were not different among eras in the first year of disease $[p=0.15$ and $p=0.71$, respectively]. In contrast, the cumulative use of CS [days and dosage] in the subsequent 4 years of disease decreased over time: from a median of 198 days [IQR 26-653]/2320 mg [IQR 158-7,487] in the era '91-'98 to a median of 0 days [IQR 0-83]/0 mg [IQR 0-1260] in the era '06-'11 [ $p<0.01$ for both outcomes]. In line, the total cumulative 5 -year CS use decreased over time $[p<0.01$ for both days and dosage analyses], see Table 5.5. 
Table 5.5. Cumulative corticosteroid use

\begin{tabular}{|c|c|c|c|c|c|}
\hline & & $\begin{array}{l}\text { Era } 1991-1998 \\
\quad[N=142]\end{array}$ & $\begin{array}{c}\text { Era 1999-2005 } \\
\quad[N=194]\end{array}$ & $\begin{array}{c}\text { Era 2006-2011 } \\
\quad[\mathrm{N}=93]\end{array}$ & \\
\hline \multicolumn{6}{|l|}{$\begin{array}{l}\text { Within first year } \\
\text { of disease }\end{array}$} \\
\hline $\begin{array}{l}\text { Median } \\
\text { cumulative } \\
\text { number of days }\end{array}$ & {$[\mathrm{IQR}]$} & 107 [35-268] & $81[13-170]$ & $79[54-160]$ & $p=0.15$ \\
\hline $\begin{array}{l}\text { Median } \\
\text { cumulative dose }\end{array}$ & {$[\mathrm{QQR}]$} & 2095 [500-4189] & 1680 [196-3460] & 1580 [935-3133] & $p=0.71$ \\
\hline \multicolumn{6}{|l|}{$\begin{array}{l}\text { Between first and } \\
\text { fifth year of disease }\end{array}$} \\
\hline $\begin{array}{l}\text { Median } \\
\text { cumulative } \\
\text { number of days }\end{array}$ & {$[\mathrm{IQR}]$} & 198 [26-653] & $63[0-173]$ & 0 [0-83] & $p<0.01$ \\
\hline $\begin{array}{l}\text { Median } \\
\text { cumulative dose }\end{array}$ & {$[\mathrm{IQR}]$} & 2319 [158-7487] & $880[0-2680]$ & $0[0-1260]$ & $p<0.01$ \\
\hline \multicolumn{6}{|l|}{$\begin{array}{l}\text { Within the first five } \\
\text { years of disease }\end{array}$} \\
\hline $\begin{array}{l}\text { Median } \\
\text { cumulative } \\
\text { number of days }\end{array}$ & {$[\mathrm{QQR}]$} & 366 [107-841] & $161[75-314]$ & $120[72-211]$ & $p<0.01$ \\
\hline $\begin{array}{l}\text { Median } \\
\text { cumulative dose }\end{array}$ & {$[\mathrm{QQR}]$} & 4643 [1828-10234] & 2970 [1508-5081] & $2180[1278-37150$ & $p<0.01$ \\
\hline
\end{tabular}

$\mathrm{IQR}$, interquartile range. Analyses were performed in patients with a corticosteroid prescription within five years after diagnosis and a minimum follow-up of five years. 


\section{Discussion}

In this real-life, population-based, Dutch IBDSL cohort, the long-term outcome of CD has improved over the past two decades and, in parallel, marked changes were observed in the medical management. Over time, the use of immunomodulators and anti-TNF $\alpha$ agents increased profoundly and these agents were prescribed earlier after CD diagnosis. The more recently diagnosed CD patients had lower hospitalization and surgery rates, both at diagnosis and on the long-term, and less days of CS exposure during their course. Although fewer patients had complicated $C D$ in more recent eras, the risk of progression from an inflammatory to a stricturing or penetrating phenotype has not changed. The use of immunomodulators or anti-TNFa agents within 2 years after CD diagnosis was not significantly associated with a lower hospitalization, phenotype progression, or surgery rate.

Nowadays, the vast majority [70.8\%] of CD patients will ever use immunomodulators and nearly half [49.5\%] starts treatment in the first year after CD diagnosis. This is in line with observations in other large cohorts ${ }^{15,17,18}$ and with current guidelines that advocate the use of

immunomodulators as first-line maintenance therapy and CS sparing agent in CD. 6,7 The immunomodulator exposure is rather high in the IBDSL cohort, as compared with other population-based cohorts from the same era, that observed 5-year exposure rates of 45$59 \% .{ }^{15,18,35}$ Nearly one-third of our CD patients diagnosed after the year of anti-TNF $\alpha$ introduction [1999] is exposed to these agents within 5 years after diagnosis that is also high as compared with other cohorts, such as the Copenhagen County cohort [23.3\% within 7 years between 2003 and 2011] $]^{3,6}$ and the ECCO-EpiCom cohort [19\% within 1 year in 2011-2012]., Increasing exposure rates of immunomodulators and, in particular, anti-TNFa agents, will inevitably lead to higher medication costs and overtreatment looms. Although total costs for IBD care have not increased over time, the Dutch COIN study recently showed that costs for IBD medication have increased and are currently the main cost driver in IBD., ${ }^{3,8}$ Future studies that aim to stratify patients in order to select the optimal treatment strategy for each CD patient are warranted to avert under- and overtreatment.

Induction and maintenance of steroid-free clinical remission is the primary treatment goal in CD, but data regarding the cumulative CS use are scarce. A Canadian population-based study [UMIBDED] found no change in cumulative CS use between 1995 and 2010, despite increasing immunomodulator use. ${ }^{31}$ The present study is the first European study including this important outcome parameter and shows a clear reduction in cumulative CS use in more recently diagnosed CD patients. This discrepancy between studies may be explained by regional differences in prescription policy, but a more profound increase in the cumulative 5 year exposure rates of immunomodulators [UMIBDED: 19.8\% [1995-2000] to 31.7\% [2005-2009] vs. IBDSL: 30.6\% [1991-1998] to 70.8\% [2006-2011]] and anti-TNFa 
agents [UMIBDED: 5.1\% [2001-2004] to 12.7\% [2005-2009] vs. IBDSL: 19.9\% [19992005] to $41.2 \%$ [2006-2010]] in our area must be acknowledged. Next, we observed that more CD patients in the era '06-' 11 than in the era ' 91 -'98 received a CS prescription within the first year after diagnosis. Th is probably results from a high adherence to recent guidelines that advocate the use of CS for remission induction for all newly diagnosed CD patients, except for patients with limited ileal disease. 6,7 The observed cumulative use in the first year of disease in the era '06-'11 [median 79 days [IQR 48-144]/1580 mg [IQR 840-3060]] corresponds to approximately one course of CS [63 days, $1505 \mathrm{mg}$ ], assuming a start dose of $40 \mathrm{mg}$ prednisolone and a standard 8-10 weeks of tapering regime as recommended in the Dutch guideline from 2009. ${ }^{6}$ Beyond the first year after diagnosis, we observed a clear reduction in the cumulative CS use in more recent eras. This may be the result of more awareness for steroid sparing in more recent years, a milder course of the disease [whether or not because of changes in medical therapy], an earlier diagnosis of the disease [and thus earlier start of treatment], or a combination of these factors.

In all eras, a considerable number of patients suffered from a complicated CD behavior. Over time, a reduction in the number of patients presenting with a complicated behavior was seen in the present study as well as in the cohort from Hungary. ${ }^{15}$ This may be explained by earlier diagnosis of $C D$, as strictures and internal fistulas are considered to be the result of longstanding inflammation and subsequent bowel damage.,3,40 Regrettably, no data on the time from first symptoms to diagnosis were available for study. The cumulative 5year probability of having a complicated CD behavior was 35.5\% in our cohort, and this is lower than reported in other population-based studies [40-55\%] $]^{1,13,14}$ and lower than in the landmark study from Cosnes et al. ${ }^{12}$ on this topic [52\%]. Of note, previous studies often used the Vienna consensus ${ }^{30}$ to classify disease behavior, in which perianal fistulas were also considered penetrating disease. When we reclassify disease behavior accordingly [Supplementary Figure 5.S1], the proportion of patients with a complicated behavior within 5 years after diagnosis is more alike [43.1\%]. Progression from B1 to either B2 or B3 disease occurred in $21 \%$ within 5 years and was similar among the three eras, despite the earlier and higher exposure to immunomodulators and anti-TNF $\alpha$ agents in more recent eras. This is of relevance, as the prevention of structural bowel damage is currently suggested as a novel therapeutic goal in CD. ${ }^{8,41}$

In parallel to an increasing use of immunomodulators and anti-TNFa agents, we observed a considerable drop in the surgery rate, both at diagnosis and during follow-up, even within the biological era. The lower surgery rate at diagnosis may be explained by a combination of improved diagnostics, disease awareness, or a change in treatment policy. A local effect is suspected, as other cohorts did not find such a decrease in the surgery rate at diagnosis. ${ }^{17,18}$ The 5-year surgery rate decreased as well, even after correcting for surgeries 
performed at diagnosis. This observation is in line with Danish data that show a decrease in the surgery rate from $44.7 \%$ in $1979-1986$ to $19.6 \%$ in 2003-201117, and with Cardiff data that show a decrease from $59 \%$ in $1986-1991$ to $25 \%$ in $1998-2003 .{ }^{18}$ The present study is the first to include the indication of surgery and shows that the drop in surgery rate is mainly caused by a reduction in surgeries for refractory inflammatory disease rather than complicated disease.

To assess whether the temporal improvements in the long-term outcome of $\mathrm{CD}$ are related to the observed changes in the medical management of $C D$, we compared the longterm outcome of immunomodulator and anti-TNF $\alpha$ users with matched controls. No significant association was found between the use of these agents in the first 2 years of disease and the subsequent hospitalization, surgery, or phenotype progression risk. Results were concordant in the sensitivity analyses in which cutoff values of 6 months and 1 year were used for the definition of "user". Therefore, the results of the present study seem to indicate that the observed improvements in the long-term outcome are mainly caused by other factors than changes in medical management, such as [local] changes in the indications for hospitalization and surgery, or disease monitoring. Referring to the latter, IBD patients are more tightly monitored nowadays: biochemically [e.g., regular checks for blood and fecal inflammation markers], radiologically [e.g., assessment of proximal disease activity and extension], and personally [e.g., easily accessible specialized IBD nurses, adherence coaching]. As a result, disease activity or disease-related complications may be recognized and treated at an earlier stage, thereby possibly preventing further progression. Previous studies reflecting on the question of whether medication can alter the natural history of IBD did find an association between the use of azathioprine and/or anti-TNF $\alpha$ and a lower surgery rate. ${ }^{15,42-}$ ${ }^{45}$ However, not all studies adequately adjusted for confounding by indication or immune time bias. Propensity score matching is commonly used to control for confounding by indication ${ }^{46}$, yet its performance depends on the quality of the variables included in the model. In IBD, accurate [clinical] predictors for the disease outcome are currently lacking, limiting the extent to which we can account for such confounding. ${ }^{47}$ As a result, the findings from the propensity score analyses may therefore be underestimated. Referring to immune time bias ${ }^{48}$, Targownik et al. ${ }^{49}$ postulated that this type of bias may explain the strong beneficial effects of immunomodulators and anti-TNF $\alpha$ agents on surgery risk or phenotype progression that were found in some observational studies. This may explain the discrepancy between the negative findings in the present study and the positive findings in others.

Ultimately, because of the complexity of establishing proper models to study the relation between treatment and outcome and the large number of patients required to measure an effect, our findings should be interpreted with care. Future studies, in particular in cohorts with more patients treated with anti-TNFa agents or combination therapy early in 
disease course, are needed to further reflect on the question whether specific treatment strategies can change the natural history of $C D$.

The strengths of this study reside in the prospective registration of newly diagnosed IBD patients in a well-defined geographical area, the large number of CD patients included and high completeness of the cohort [over 93\%] $]^{28}$, the long follow-up time, and the level of detail of the clinical data directly retrieved from patients' files rather than from administrative databases. This enabled us to study time trends in medical management and outcomes in an unselected CD population in real-life clinical practice. Nonetheless, several limitations have to be addressed. First, because of the observational design of the study, no causal relations can be claimed. Second, data on the diagnostic delay would have provided insight into the observed changes in disease presentation, but were not available and are difficult to obtain because of recall bias. Third, hospitalization, surgery, and cumulative CS use were used as proxy measures for a severe disease outcome. Clinical and endoscopic disease activity scores and biochemical markers would have provided additional insight, yet are very hard to collect and interpret in large, observational cohort studies. Finally, data on the cumulative CS use were retrieved from medical records rather than pharmacies, inducing a certain amount of inaccuracy.

In conclusion, the hospitalization and surgery rates decreased over the past two decades, whereas progression to complicated disease is still common in CD. The improvements were not significantly associated with the use of immunomodulators or antiTNF $\alpha$ agents. Future studies should address whether novel treatment strategies, such as treat to target, can further improve the long-term outcome, in particular the risk of developing structural bowel damage. 


\section{Supplement}

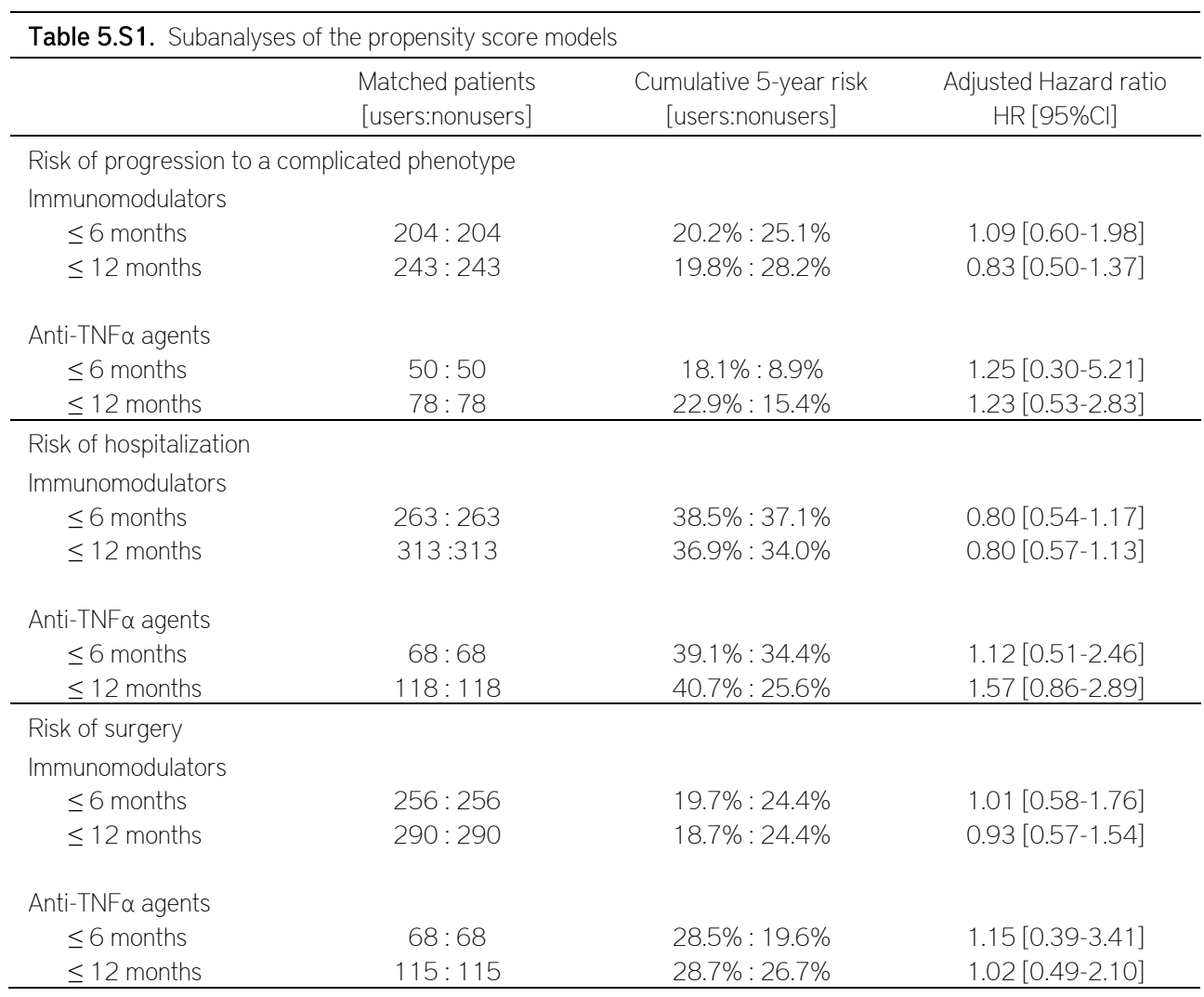




\section{Distribution of disease behavior over course}

\section{(Vienna classification)}

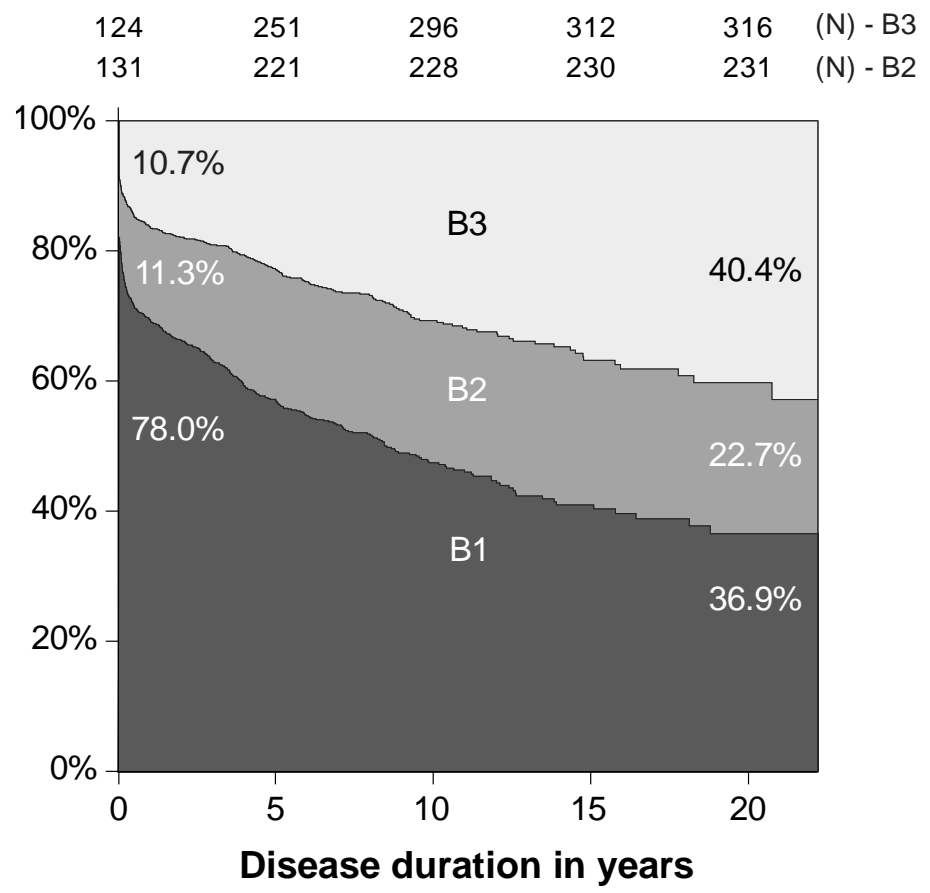

Figure 5.S1. Distribution of disease behavior over course [Vienna classification].

$\mathrm{N}$, number of patients who have the specific disease phenotype. B1, nonstricturing, nonpenetrating disease; B2, stricturing disease; B3, penetrating disease. 


\section{References}

1. Solberg IC, Vatn $\mathrm{MH}$, Hoie $\mathrm{O}$, et al. Clinical course in Crohn's disease: results of a Norwegian population-based tenyear follow-up study. Clin Gastroenterol Hepatol 2007;5:1430-8.

2. Wolters FL, Russel MG, Sijbrandij J, et al. Phenotype at diagnosis predicts recurrence rates in Crohn's disease. Gut 2006;55:1124-30.

3. Silverstein MD, Loftus EV, Sandborn WJ, et al. Clinical course and costs of care for Crohn's disease: Markov model analysis of a population-based cohort. Gastroenterology 1999;117:49-57.

4. Faubion WA, Jr., Loftus EV, Jr., Harmsen WS, et al. The natural history of corticosteroid therapy for inflammatory bowel disease: a population-based study. Gastroenterology 2001;121:255-60.

5. Zallot C, Peyrin-Biroulet L. Clinical risk factors for complicated disease: how reliable are they? Dig Dis 2012;30 Suppl 3:67-72.

6. van Bodegraven $A A$, van Everdingen $\mathrm{JJ}$, Dijkstra G, et al. [Guideline 'Diagnosis and treatment of inflammatory bowel disease in adults'. I. Diagnosis and treatment]. Ned Tijdschr Geneeskd 2010;154:A1899.

7. Dignass A, Van Assche G, Lindsay JO, et al. The second European evidence-based Consensus on the diagnosis and management of Crohn's disease: Current management. J Crohns Colitis 2010;4:28-62.

8. Peyrin-Biroulet L, Sandborn W, Sands BE, et al. Selecting Therapeutic Targets in Inflammatory Bowel Disease [STRIDE]: Determining Therapeutic Goals for Treat-to-Target. Am J Gastroentero/2015;110:1324-38.

9. Szklo M. Population-based cohort studies. Epidemiol Rev 1998;20:81-90.

10. Gower-Rousseau C, Savoye G, Colombel $\mathrm{JF}$, et al. Are we improving disease outcomes in IBD? A view from the epidemiology side. Gut 2014;63:152930.

11. Peyrin-Biroulet L, Loftus EV, Jr., Colombel JF, et al. The natural history of adult Crohn's disease in populationbased cohorts. Am J Gastroenterol 2010;105:289-97.

12. Cosnes J, Cattan S, Blain A, et al. Longterm evolution of disease behavior of Crohn's disease. Inflamm Bowel Dis 2002;8:244-50.

13. Tarrant KM, Barclay ML, Frampton CM, et al. Perianal disease predicts changes in Crohn's disease phenotype-results of a population-based study of inflammatory bowel disease phenotype. Am J Gastroentero/ 2008;103:308293.

14. Lovasz BD, Lakatos L, Horvath A, et al. Evolution of disease phenotype in adult and pediatric onset Crohn's disease in a population-based cohort. World J Gastroenterol2013;19:2217-26.

15. Lakatos PL, Golovics PA, David G, et al. Has there been a change in the natural history of Crohn's disease? Surgical rates and medical management in a population-based inception cohort from Western Hungary between 1977-2009. Am J Gastroentero/ 2012;107:579-88.

16. Nguyen $G C$, Nugent $Z$, Shaw $S$, et al. Outcomes of patients with Crohn's disease improved from 1988 to 2008 and were associated with increased specialist care. Gastroenterology 2011;141:90-7.

17. Rungoe $C$, Langholz E, Andersson M, et al. Changes in medical treatment and surgery rates in inflammatory bowel disease: a nationwide cohort study 1979-2011. Gut 2014;63:1607-16.

18. Ramadas AV, Gunesh S, Thomas GA, et al. Natural history of Crohn's disease in a population-based cohort from Cardiff [1986-2003]: a study of changes in medical treatment and surgical resection rates. Gut 2010;59:1200-6. 
19. Targan SR, Hanauer SB, van Deventer SJ, et al. A short-term study of chimeric monoclonal antibody cA2 to tumor necrosis factor alpha for Crohn's disease. Crohn's Disease cA2 Study Group. N Engl J Med 1997;337:102935.

20. Hanauer SB, Feagan BG, Lichtenstein $\mathrm{GR}$, et al. Maintenance infliximab for Crohn's disease: the ACCENT I randomised trial. Lancet 2002;359:1541-9.

21. Hanauer SB, Sandborn WJ, Rutgeerts $P$, et al. Human anti-tumor necrosis factor monoclonal antibody [adalimumab] in Crohn's disease: the CLASSIC-I trial. Gastroenterology 2006;130:323-33; quiz 591.

22. Colombel JF, Sandborn WJ, Rutgeerts $P$, et al. Adalimumab for maintenance of clinical response and remission in patients with Crohn's disease: the CHARM trial. Gastroenterology 2007;132:52-65.

23. Akobeng AK, Zachos M. Tumor necrosis factor-alpha antibody for induction of remission in Crohn's disease. Cochrane Database Syst Rev 2004:CD003574.

24. Behm BW, Bickston SJ. Tumor necrosis factor-alpha antibody for maintenance of remission in Crohn's disease. Cochrane Database Syst Rev 2008:CD006893.

25. Chande N, Tsoulis DJ, MacDonald JK. Azathioprine or 6-mercaptopurine for induction of remission in Crohn's disease. Cochrane Database Syst Rev 2013;4:CD000545.

26. Chande N, Patton PH, Tsoulis DJ, et al. Azathioprine or 6-mercaptopurine for maintenance of remission in Crohn's disease. Cochrane Database Syst Rev 2015:10:CD000067.

27. Centraal Bureau voor de Statistiek. CBS StatLine - Bevolkingsontwikkeling; levendgeborenen, overledenen en migratie per regio. In; 2015.

28. van den Heuvel TR, Jonkers DM, Jeuring SF, et al. Cohort Profile: The Inflammatory Bowel Disease South
Limburg Cohort [IBDSL]. Int J Epidemiol 2015; pii:dyv088.

29. Satsangi J, Silverberg MS, Vermeire S, et al. The Montreal classification of inflammatory bowel disease: controversies, consensus, and implications. Gut 2006;55:749-53.

30. Gasche C, Scholmerich J, Brynskov J, et al. A simple classification of Crohn's disease: report of the Working Party for the World Congresses of Gastroenterology, Vienna 1998. Inflamm Bowel Dis 2000;6:8-15.

31. Targownik LE, Nugent $Z$, Singh $H$, et al. Prevalence of and outcomes associated with corticosteroid prescription in inflammatory bowel disease. Inflamm Bowel Dis 2014;20:622-30.

32. Kleinbaum DG, Klein M. Survival Analysis: A Self-Learning Text. 3rd edition ed: Springer-Verlag New York; 2012.

33. Walker AM. Confounding by indication. Epidemiology 1996;7:335-6.

34. Suissa S. Immortal time bias in pharmaco-epidemiology. Am J Epidemiol 2008; 167:492-9.

35. Vester-Andersen MK, Vind I, Prosberg $\mathrm{MV}$, et al. Hospitalisation, surgical and medical recurrence rates in inflammatory bowel disease 20032011-a Danish population-based cohort study. J Crohns Colitis 2014;8:167583.

36. Vester-Andersen MK, Prosberg MV, Jess $T$, et al. Disease course and surgery rates in inflammatory bowel disease: a population-based, 7-year follow-up study in the era of immunomodulating therapy. Am J Gastroenterol 2014;109:705-14.

37. Vegh Z, Burisch J, Pedersen N, et al. Treatment Steps, Surgery, and Hospitalization Rates During the First Year of Follow-up in Patients with Inflammatory Bowel Diseases from the 2011 ECCO-Epicom Inception Cohort. J Crohns Colitis 2015;9:747-53.

38. van der Valk ME, Mangen MJ, Leenders $\mathrm{M}$, et al. Healthcare costs of inflammatory bowel disease have shifted 
from hospitalisation and surgery

towards anti-TNFalpha therapy: results from the COIN study. Gut2014;63:729.

39. Rieder F, Zimmermann EM, Remzi FH, et al. Crohn's disease complicated by strictures: a systematic review. Gut 2013;62:1072-84.

40. Scharl M, Rogler G. Pathophysiology of fistula formation in Crohn's disease. World J Gastrointest Pathophysiol 2014;5:205-12.

41. Allen PB, Peyrin-Biroulet L. Moving towards disease modification in inflammatory bowel disease therapy. Curr Opin Gastroenterol2013;29:397404.

42. Picco MF, Zubiaurre I, Adluni M, et al. Immunomodulators are associated with a lower risk of first surgery among patients with non-penetrating nonstricturing Crohn's disease. Am J Gastroentero/2009;104:2754-9.

43. Peyrin-Biroulet L, Oussalah A, Williet N, et al. Impact of azathioprine and tumour necrosis factor antagonists on the need for surgery in newly diagnosed Crohn's disease. Gut 2011;60:930-6.

44. Kariyawasam VC, Selinger CP, Katelaris $\mathrm{PH}$, et al. Early use of thiopurines or methotrexate reduces major abdominal and perianal surgery in Crohn's disease. Inflamm Bowel Dis 2014;20:1382-90.

45. Safroneeva E, Vavricka SR, Fournier N, et al. Impact of the early use of immunomodulators or TNF antagonists on bowel damage and surgery in Crohn's disease. Aliment Pharmacol Ther 2015;42:977-89.

46. Wise L. Risks and benefits of [pharmaco]epidemiology. Ther Adv Drug Saf 2011:2:95-102.

47. Torres J, Caprioli F, Katsanos KH, et al. Predicting Outcomes to Optimize Disease Management in Inflammatory Bowel Diseases. J Crohns Colitis 2016;10:1385-94.

48. Suissa S. Immortal time bias in observational studies of drug effects.
Pharmacoepidemiol Drug Saf

2007;16:241-9.

49. Targownik LE, Suissa S. Understanding and Avoiding Immortal-Time Bias in Gastrointestinal Observational Research. Am J Gastroenterol 2015:110:164750 



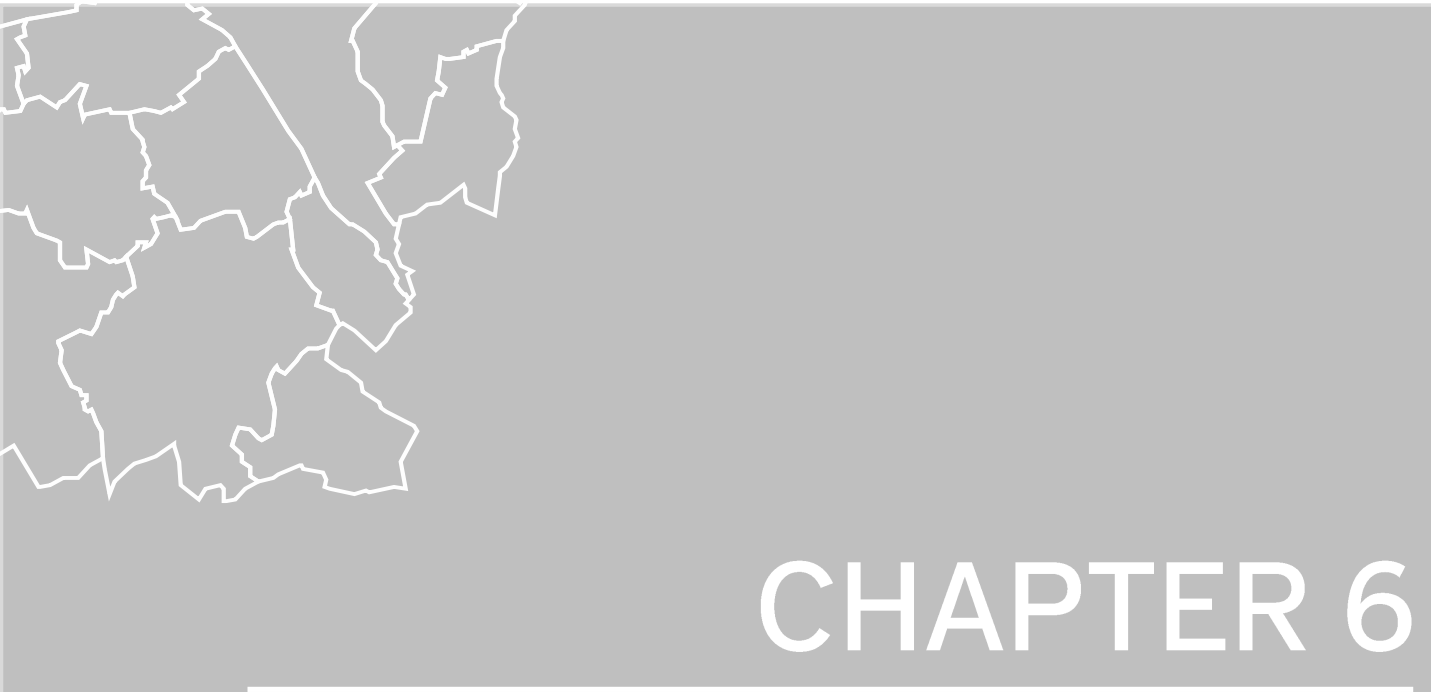

\section{Disease outcome of ulcerative colitis in an era of changing treatment strategies}

Steven FG Jeuring, Paul HA Bours, Maurice P Zeegers, Ton W van Ambergen, Tim RA van den Heuvel, Marielle JL Romberg-Camps, Ad A van Bodegraven, Liekele E Oostenbrug, Stephanie O Breukink, Laurents PS Stassen, Wim H Hameeteman, Ad AM Masclee, Daisy MAE Jonkers, Marieke J Pierik

J Crohns Colitis. 2015 Oct;9(10):837-45 


\section{Abstract}

\section{Background}

In the past decades, treatment options and strategies for ulcerative colitis [UC] have radically changed. Whether these developments have altered the disease outcome at population level is yet unknown. Therefore, we evaluated the disease outcome of UC over the past two decades in the South Limburg area of the Netherlands.

\section{Methods}

In the Dutch population-based IBDSL cohort, three time cohorts were defined: cohort 1991 1997 [cohort A], cohort 1998-2005 [cohort B], and cohort 2006-2010 [cohort C]. The colectomy and hospitalization rates were compared between cohorts by Kaplan-Meier survival analyses. Hazard ratios [HR] for early colectomy [within 6 months after diagnosis], late colectomy [beyond 6 months after diagnosis], and hospitalization were calculated using Cox regression models.

\section{Results}

In total, 476 UC patients were included in cohort A, 587 patients in cohort B, and 598 patients in cohort C. Over time, an increase in the use of immunomodulators [8.1\%, $22.8 \%$ and $21.7 \%$, respectively, $p<0.01]$ and biological agents [0\%, $4.3 \%$ and $10.6 \%$, respectively, $p<0.01$ ] was observed. The early colectomy rate decreased from $1.5 \%$ in cohort A to $0.5 \%$ in cohort B [HR 0.14; 95\% confidence interval 0.04-0.47], with no further decrease in cohort C [0.3\%, HR 0.98; $95 \%$ confidence interval 0.20-4.85]. Late colectomy rate remained unchanged over time $[4.0 \%$ vs $5.2 \%$ vs $3.6 \%$, respectively, $p=0.54]$. Hospitalization rate was also similar among cohorts [ $22.3 \%$ vs $19.5 \%$ vs $18.3 \%$, respectively, $p=0.10$ ].

\section{Conclusion}

Over the past two decades, a reduction in early colectomy rate was observed, with no further reduction in the most recent era. Late colectomy rate and hospitalization rate remained unchanged over time. 


\section{Introduction}

Ulcerative colitis [UC] is an invalidating, chronic inflammatory disease restricted to the colon. The disease course of UC is heterogeneous, ranging from long-term quiescent disease to fulminant, therapy-refractory disease necessitating rescue surgery. Population-based cohort studies report that $8-24 \%$ of UC patients ultimately need a colectomy, the majority within 2 years after diagnosis. ${ }^{1-7}$ Colectomy with or without ileal pouch-anal reconstruction [IPAA] is an effective treatment for UC, but perioperative complications are frequently encountered. ${ }^{8}$ Postoperatively, the procedure is associated with lower fecundity, pouchitis and a decreased quality of life due to invalidating complaints such as frequent stools, urgency, and soiling. 9,10 Therefore, prevention of colectomy is an important goal in UC management.

In the past decades, treatment strategies for UC have changed. Immunomodulators are more frequently used and are given earlier in UC disease course nowadays. ${ }^{11-13}$ In 2006, the therapeutic armamentarium extended with the registration of biological therapy for $U C$ in the Netherlands. Both immunomodulators ${ }^{14-17}$ and anti-tumor necrosis factor alpha [TNF $\alpha$ ] agents ${ }^{18-20}$ are effective in inducing and maintaining clinical remission in UC. In addition, followup data from the Active Ulcerative Colitis Trials [ACT] showed a lower colectomy rate in the infliximab [IFX] group [10\%] compared with the placebo group [17\%] at week 54 in a trial population. ${ }^{21}$ Recently, a decline in the annual colectomy rate was observed in a Canadian cohort after the year of registration of IFX, suggesting an effect of biological availability on UC disease outcome. ${ }^{22}$ For immunomodulators, only an association between early use and a decreased risk of surgery was found in Crohn's disease. ${ }^{23-24}$ Data in UC on this topic are lacking. Despite the well-described efficacy of current treatment modalities in trial populations, little is known about the real-life disease outcome of UC patients diagnosed in the current era, in which early use of immunomodulators is incorporated in guidelines and biological therapy is available.

The aim of this study was to evaluate the disease outcome of UC patients diagnosed in the current era at population level, and to gain insight into the evolution of the colectomy and hospitalization rates over time. 


\section{Methods}

\section{Cohort description}

The IBD South Limburg [IBDSL] cohort is a well-characterized population-based inflammatory bowel disease [IBD] cohort in the South Limburg area of the Netherlands. ${ }^{25}$ Previous studies reported on the incidence of IBD in this area. ${ }^{26-27}$ South Limburg is a well-defined geographical entity located in the south-east of the Netherlands. It is bordered by Germany and Belgium in the east and south-west, and its northern border with the central part of the Netherlands is narrow. Between 1991 and 2010, the average population in South Limburg was approximately $635,000 .{ }^{28}$ Migration in and out of the area is low, with a net migration rate of 2.1 per 1000 inhabitants per year. ${ }^{28}$ The area comprises two general hospitals [Atrium Medical Center in Heerlen and Orbis Medical Center in Sittard] and one academic referral hospital [Maastricht University Medical Center [MUMC+] in Maastricht] providing almost all endoscopic gastroenterological services in the area.

From 1991, incident adult IBD patients diagnosed in the South Limburg area are registered in IBDSL. Newly-diagnosed IBD patients are identified via hospital administration, diagnosis-treatment combination codes [Dutch variant of the case-mix reimbursement system applied in several other countries $\left.{ }^{29}\right]$, and by a search in the nationwide digital pathology database [PALGA]. These registrations are reviewed based on the assumption that in-hospital diagnostics [endoscopy or imaging] are needed to establish IBD diagnosis. To assure its population-based character and its completeness, the IBDSL registry has been cross-checked with IBD patients present in patient registries from local GPs. This check indicated that 93\% of all eligible IBD patients living in the South Limburg area are actually included in the IBDSL cohort. ${ }^{25}$ This study was approved for all centers by the Medical Ethics Committee of the Maastricht University Medical Center [NL31636.068.10] and registered in ClinicalTrial. gov [NCT02130349].

\section{Study population and design}

All UC patients diagnosed between January 1, 1991 and December 31, 2010 were eligible for inclusion in the present study. Exclusion criteria were an age at diagnosis under 18 years, living outside South Limburg, and a previous diagnosis of Crohn's disease [CD], unclassified colitis [IBD-U], or indeterminate colitis [IBD-I]. Demographic data, disease extent, medication use, and the dates of hospitalization and colectomy were retrieved from medical records, using standardized registration forms.

To assess changes in the colectomy and hospitalization rates, three time cohorts were composed, based on date of diagnosis: cohort 1991-1997, cohort 1998-2005, and cohort 2006-2010. The latter cohort reflects the biological era, as the first anti-TNF $\alpha$ agent 
registered for UC [IFX] was available in the Netherlands as from 2006. The pre-biological era was equally divided into two time periods. Patients were followed until last visit, date of migration out of the area, death, or end of data collection [December 31, 2011], whichever came first.

\section{Definitions}

The diagnosis of UC was based on the combination of clinical, endoscopic, or radiological findings in conjunction with histological findings as described by Lennard-Jones. ${ }^{30}$ The date of the first endoscopy with typical mucosal inflammation was used as date of diagnosis. Disease location at diagnosis was classified as ulcerative proctitis [E1], left-sided UC [E2], and extensive UC [E3]..$^{31}$ Use of steroids was defined as the use of any systemic corticosteroid orally or intravenously administered. Under this definition, budesonide was not considered systemic steroid treatment. Thiopurines comprised azathioprine, 6-mercaptopurine, and tioguanine. These agents were available during the complete time period of the present cohort and were gradually adopted in UC management in the 1990s. Thiopurines have been used in UC patients with steroid-dependent disease as well as in patients treated with ciclosporin for acute severe colitis. The 2008 and 2012 European guidelines ${ }^{32-33}$ and 2009 Dutch guidelines ${ }^{34}$ on UC management have been advocated in the Netherlands ever since their availability. According to these guidelines, anti-TNF $\alpha$ therapy is indicated in patients with moderately active $U C$ refractory or intolerant to thiopurines, patients with moderately active steroid-refractory UC, and in patients with acute severe colitis failing intravenous corticosteroids [rescue therapy]. Acute severe disease and chronic active disease [steroiddependent or steroid-refractory] were defined according to the criteria from the European Crohn's and Colitis Organisation. ${ }^{35}$ Rescue therapy was defined as the administration of ciclosporin or IFX in patients with an acute severe colitis failing intravenous corticosteroids.

\section{Outcome measures}

Outcome measures of this study were medication use, occurrence of hospitalization, and occurrence of colectomy. Colectomy was defined as colectomy with ileostomy, proctocolectomy with ileostomy, or colectomy with IPAA. If colectomy was performed within 6 months after diagnosis, we considered it as 'early colectomy'; operation beyond 6 months after diagnosis was considered to be 'late colectomy'. Hospitalization was defined as a hospital admission due to UC-related complaints [first presentation or flare], UC-related surgery, or a combination of both. Short hospital admissions for IFX infusions or clinical colonoscopies only were excluded. 


\section{Statistical analyses}

Data were presented as median with interquartile ranges [IQR], or as mean with standard deviation [SD], depending on normality of the underlying distribution. Continuous data were compared by an independent Student's t-test or a Kruskal-Wallis test in case of nonparametric data. Dichotomous data were compared by Chi-square tests. A Kaplan-Meier survival analysis was used to estimate the cumulative proportion of patients who used immunomodulators or anti-TNF $\alpha$ agents, underwent colectomy, or were hospitalized 1, 2, and 5 years following diagnosis, so that differences in follow-up between patients were taken into account. Differences between groups were assessed by log-rank test [presented are the corresponding $p$-values] and by Cox regression analyses. A multivariable Cox regression model was used to compare medication use and the colectomy and hospitalization rates between the three time cohorts. Disease extent at diagnosis, sex, and age at diagnosis were also included in the multivariable model, to adjust for possible confounding due to differences in disease prognosis or baseline characteristics between groups. Differences in hazards between groups were presented as hazard ratios [HR] with 95\% confidence intervals [Cl]. Two separate Cox models were created: one model to assess colectomy risk from date of diagnosis to 6 months after diagnosis [i.e. early colectomy] and another model to assess colectomy risk beyond 6 months after diagnosis [i.e. late colectomy], because the proportional hazards assumption was violated, indicating that the hazard ratio of colectomy was dependent on disease duration. One model estimating colectomy risk for the total follow-up period would have led to an underestimation of the early colectomy risk and an overestimation of the late colectomy risk. ${ }^{36}$ All statistical analyses were performed using SPSS Version 21 [SPSS, Chicago, IL, USA] for Windows. 


\section{Results}

\section{Study population}

From January 1991 to December 2010, 1661 patients were diagnosed with UC in South Limburg, of whom 492 [29.6\%] were in the MUMC+, 700 [42.1\%] in the Atrium Medical Center, and 469 [28.2\%] in the Orbis Medical Center.

In total, 1661 UC patients were analyzed; 476 patients were diagnosed between 1991 and 1997, 587 patients between 1998 and 2005 and 598 patients between 2006 and 2010. Median disease durations were 17.5 [IQR 15.5-19.3], 9.5 [IQR 7.6-11.5], and 3.3 [IQR 2.0-4.7] years, respectively. The main characteristics of the patients are outlined in Table 6.1.

\begin{tabular}{|c|c|c|c|c|c|}
\hline & & $\begin{array}{c}\text { Cohort } \\
1991-1997 \\
{[N=476]}\end{array}$ & $\begin{array}{c}\text { Cohort } \\
1998-2005 \\
{[N=587]}\end{array}$ & $\begin{array}{c}\text { Cohort } \\
2006-2010 \\
{[N=598]}\end{array}$ & \\
\hline Age in years ${ }^{a}$ & mean [SD] & $43.0[15.8]$ & $45.6[16.3]$ & $48.2[17.4]$ & $p<0.01$ \\
\hline Male & $N[\%]$ & $265[55.7]$ & $323[55.0]$ & $297[49.7]$ & $p=0.09$ \\
\hline Disease duration in years & median [IQR] & $17.5[15.5-19.3]$ & 9.5 [7.6-11.5] & $3.3[2.0-4.7]$ & $p<0.01$ \\
\hline Disease location ${ }^{\mathrm{a}}$ & & & & & $p<0.01$ \\
\hline E1 - proctitis & $N[\%]$ & $142[30.5]$ & 189 [32.3] & $232[38.8]$ & \\
\hline E2 - left-sided & $N[\%]$ & $251[54.0]$ & $289[49.4]$ & 249 [41.6] & \\
\hline E3 - extensive & $N[\%]$ & $72[15.5]$ & $107[18.3]$ & $117[19.6]$ & \\
\hline \multicolumn{6}{|l|}{ Medication ever used ${ }^{b}$} \\
\hline mesalazine & $N$ [\%] & 453 [98.9] & 573 [97.8] & 577 [96.5] & $p=0.04$ \\
\hline corticosteroids & $N[\%]$ & $237[51.7]$ & $276[47.1]$ & $395[66.1]$ & $p<0.01$ \\
\hline immunomodulators & $\mathrm{N}[\%]$ & $36[8.1]$ & $131[22.8]$ & $98[21.7]$ & $p<0.01$ \\
\hline ciclosporin & $\mathrm{N}$ [\%] & 18 [3.9] & 20 [3.4] & 1 [0.2] & $p<0.01$ \\
\hline anti-TNF $\alpha$ & $\mathrm{N}$ [\%] & $0[0]$ & $25[4.4]$ & 52 [10.6] & $p<0.01$ \\
\hline
\end{tabular}

$\mathrm{SD}$, standard deviation; IQR, interquartile range

${ }^{a}$ at diagnosis. Disease location could not be retrieved in 13 cases.

${ }^{\mathrm{b}}$ medication ever used was determined at 5-year follow-up

\section{Medication use}

Thiopurines were frequently used by patients in all cohorts, but an increase in the number of patients on thiopurine treatment was observed between cohorts [Figure 6.1A]. 
A

\section{Cumulative exposure to immunomodulators}
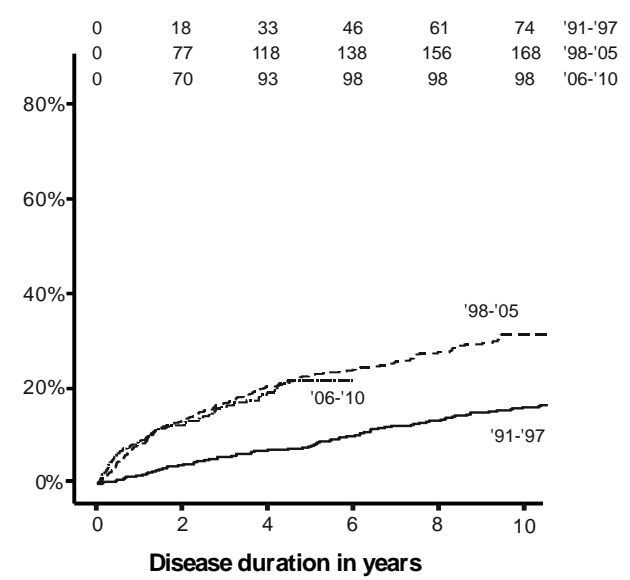

Nat risk 464

$\mathrm{N}$ at risk 584

$\mathrm{N}$ at risk 598
B

\section{Cumulative exposure to anti-TNF $\alpha$ agents}

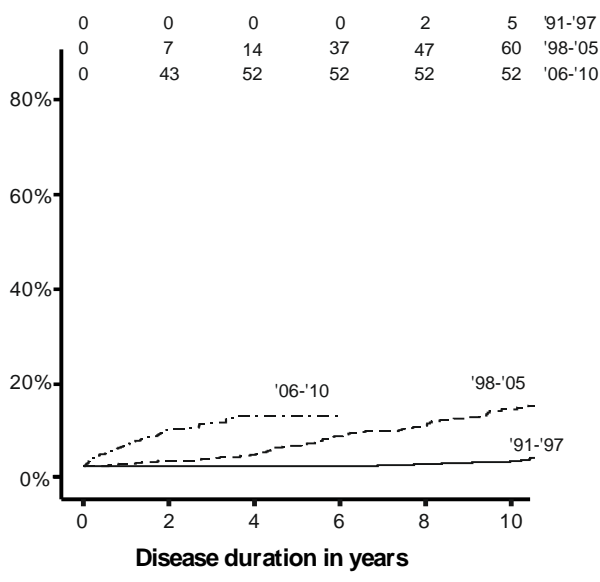

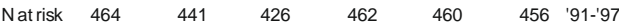

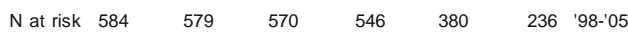
$\begin{array}{lllllll}\mathrm{N} \text { at risk } & 598 & 408 & 214 & 0 & 0 & 0\end{array}$

Figure 6.1. The cumulative use of immunomodulators $[\mathrm{A}]$ and anti-TNF $\alpha$ agents $[\mathrm{B}]$ in ulcerative colitis patients among the three cohorts.

N, number of patients who used the specific therapy. Cohort '91-'97, patients diagnosed between 1991 and 1997; cohort '98-'05, patients diagnosed between 1998 and 2005; cohort '06-'11, patients diagnosed between 2006 and 2011.

The cumulative 5-year probability of using thiopurines was $8.1 \%$ in cohort ' $91{ }^{-}{ }^{\prime} 97,22.8 \%$ in cohort '98-'05, and 21.7\% in cohort '06-'10. In contrast to the first cohort, a 2.2-fold increase in thiopurine use was observed in the second [adjusted HR 2.15;95\% Cl 1.65-2.80] and a 2.4-fold increase in the third cohort [adjusted HR 2.38; 95\% Cl 1.73-3.26]. Between the two more recent cohorts, no significant difference was observed in the proportion of thiopurine users [adjusted HR 1.11; 95\% Cl 0.85-1.44]. Over time, a difference in the timing of initiating thiopurine treatment was also observed. Within a follow-up of 5 years, time to first prescription of thiopurine medication gradually reduced from a median of 23.3 months [IQR 12.9 - 38.6] in cohort '91-'97 to a median of 16.9 months [IQR 7.5-36.5] in cohort '98-'05, to a median of 10.2 months [IQR 3.1 - 26.3] in cohort '06-'10, $p<0.01$. Within the predefined time window of early colectomy [between diagnosis and 6 months thereafter], $0.9 \%$, $4.7 \%$, and $6.4 \%$ of all UC patients were already on thiopurine treatment in the three consecutive time cohorts, respectively.

Anti-TNF $\alpha$ therapy was registered in the Netherlands in 2006, so that biological therapy was available as from diagnosis only in the last cohort. The cumulative 5-year probability of using anti-TNF $\alpha$ therapy increased from 0\% in cohort ' 91 -' $^{\prime} 7$ to $4.3 \%$ in cohort 
'98-'05, to 10.6\% in cohort '06-'10 [Figure 6.1B]. The median time from diagnosis to first IFX infusion was 44.0 months [IQR 20.9-52.1] in the second and 12.2 months [IQR 3.9-22.3] in the most recent cohort, $p<0.01$. The majority of patients in cohort '06-' 10 who underwent colectomy had failed IFX therapy [72.2\%]. Anti-TNF $\alpha$ use within 6 months after diagnosis was uncommon; no patient from cohort ' 98 -' $^{-05}$ and only $2.7 \%$ of the patients from cohort '06-'10 used biological therapy early in their disease course. The majority of patients [79.2\%] on anti-TNF $\alpha$ treatment had previously used thiopurine treatment. Combination therapy of anti-TNF $\alpha$ and a thiopurine was initially given to 29 patients [37.7\%], but the thiopurine was discontinued during follow-up in 16 patients [55.2\%], in 56.3\% of these due to adverse effects and in $43.7 \%$ to quiescent disease.

Rescue therapy was prescribed in 21 patients from cohort ' 91 -' 97,28 patients from cohort '98-'05, and 13 patients from cohort '06-'10. Corresponding 5-year cumulative probability rates of receiving rescue therapy were $1.7 \%, 3.1 \%$, and $4.1 \%$, respectively. Within the time window of early colectomy, no difference in the prevalence of rescue therapy was observed between the first two cohorts: $0.9 \%[n=6]$ vs $1.0 \%[n=6], p=0.68$, whereas no rescue therapy was used within 6 months in the most recent cohort. Ciclosporin was the common drug for rescue therapy before the availability of IFX, as it was given in $81.0 \%$ and

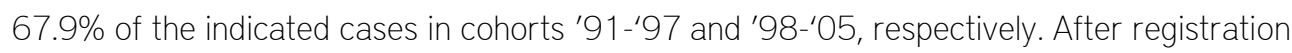
of IFX, this anti-TNFa agent was most commonly used as rescue treatment in our area, reflected by the fact that all but one patient were given IFX as rescue therapy in cohort '06-'10.

\section{Colectomy}

The cumulative colectomy rate for all three cohorts is shown in Figure 6.2. In cohort '91-'97, 51 patients underwent colectomy and the cumulative probability of undergoing colectomy after 1,2 , and 5 years was $4.1 \%, 5.6 \%$, and $7.5 \%$, respectively. In cohort ' $98-{ }^{\prime} 05,43$ patients underwent colectomy and the accompanying cumulative colectomy rate was $0.9 \%, 2.1 \%$, and $5.7 \%$, respectively. In the most recent cohort, 18 patients underwent colectomy and the accompanying cumulative colectomy rate in this cohort was $1.0 \%, 2.8 \%$, and $4.1 \%$ after 1 , 2 , and 5 years, respectively. The indications for colectomy did not differ between cohorts [ $p=0.90]$ and are shown in Table 6.2. Analyses were performed separately for early colectomy [i.e. within 6 months after diagnosis] and late colectomy [i.e. colectomy beyond 6 months after diagnosis] and results are shown in Table 6.3. 


\section{Cumulative risk of colectomy}

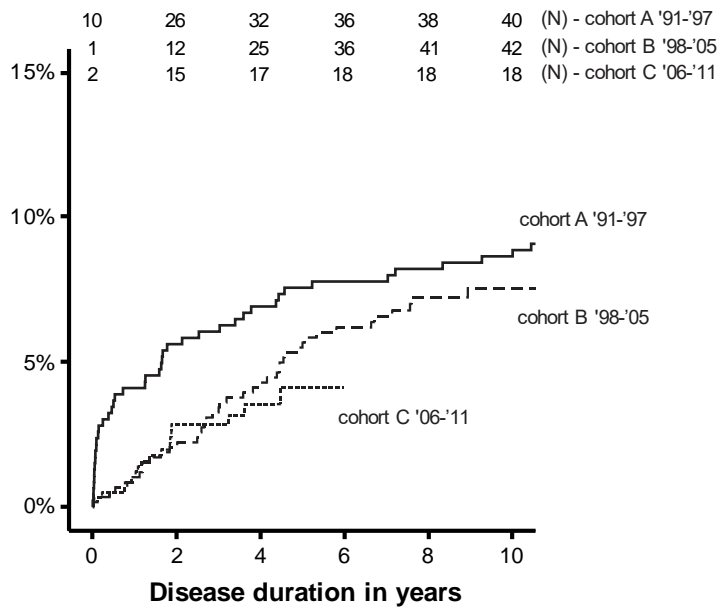

$\begin{array}{llllcccc}\mathrm{N} \text { at risk } & 464 & 438 & 432 & 428 & 426 & 423 \text { cohort A '91-'97 } \\ \mathrm{N} \text { at risk } & 584 & 572 & 557 & 544 & 372 & 233 \text { cohort B '98-'05 } \\ \mathrm{N} \text { at risk } & 598 & 432 & 225 & 0 & 0 & 0 & \text { cohort C '06-'11 }\end{array}$

Figure 6.2. The cumulative risk of colectomy in ulcerative colitis patients among the three cohorts.

$\mathrm{N}$, number of patients who underwent colectomy.

\begin{tabular}{|c|c|c|c|c|}
\hline & & $\begin{array}{c}\text { Cohort A } \\
1991-1997[\mathrm{~N}=476]\end{array}$ & $\begin{array}{c}\text { Cohort B } \\
1998-2005[\mathrm{~N}=587]\end{array}$ & $\begin{array}{c}\text { Cohort C } \\
2006-2010[N=598]\end{array}$ \\
\hline $\begin{array}{l}\text { Early colectomy } \\
\text { acute severe colitis } \\
\text { chronic active disease } \\
\text { unknown }\end{array}$ & $\begin{array}{c}N \\
N[\%] \\
N[\%] \\
N[\%]\end{array}$ & $\begin{array}{c}17 \\
13[76.5] \\
1[5.9] \\
3[17.6]\end{array}$ & $\begin{array}{c}3 \\
3[100] \\
- \\
-\end{array}$ & $\begin{array}{c}3 \\
3[100] \\
- \\
-\end{array}$ \\
\hline $\begin{array}{l}\text { Late colectomy } \\
\text { acute severe colitis } \\
\text { chronic active disease } \\
\text { colorectal malignancy } \\
\text { other } \\
\text { unknown }\end{array}$ & $\begin{array}{c}N \\
N[\%] \\
N[\%] \\
N[\%] \\
N[\%] \\
N[\%]\end{array}$ & $\begin{array}{c}34 \\
13[38.2] \\
12[35.3] \\
1[2.9] \\
1[2.9] \\
7[20.6] \\
\end{array}$ & $\begin{array}{c}40 \\
16[40.0] \\
15[37.5] \\
1[2.5] \\
3[7.5] \\
5[12.5]\end{array}$ & $\begin{array}{c}15 \\
9[60.0] \\
6[40.0] \\
- \\
- \\
-\end{array}$ \\
\hline
\end{tabular}




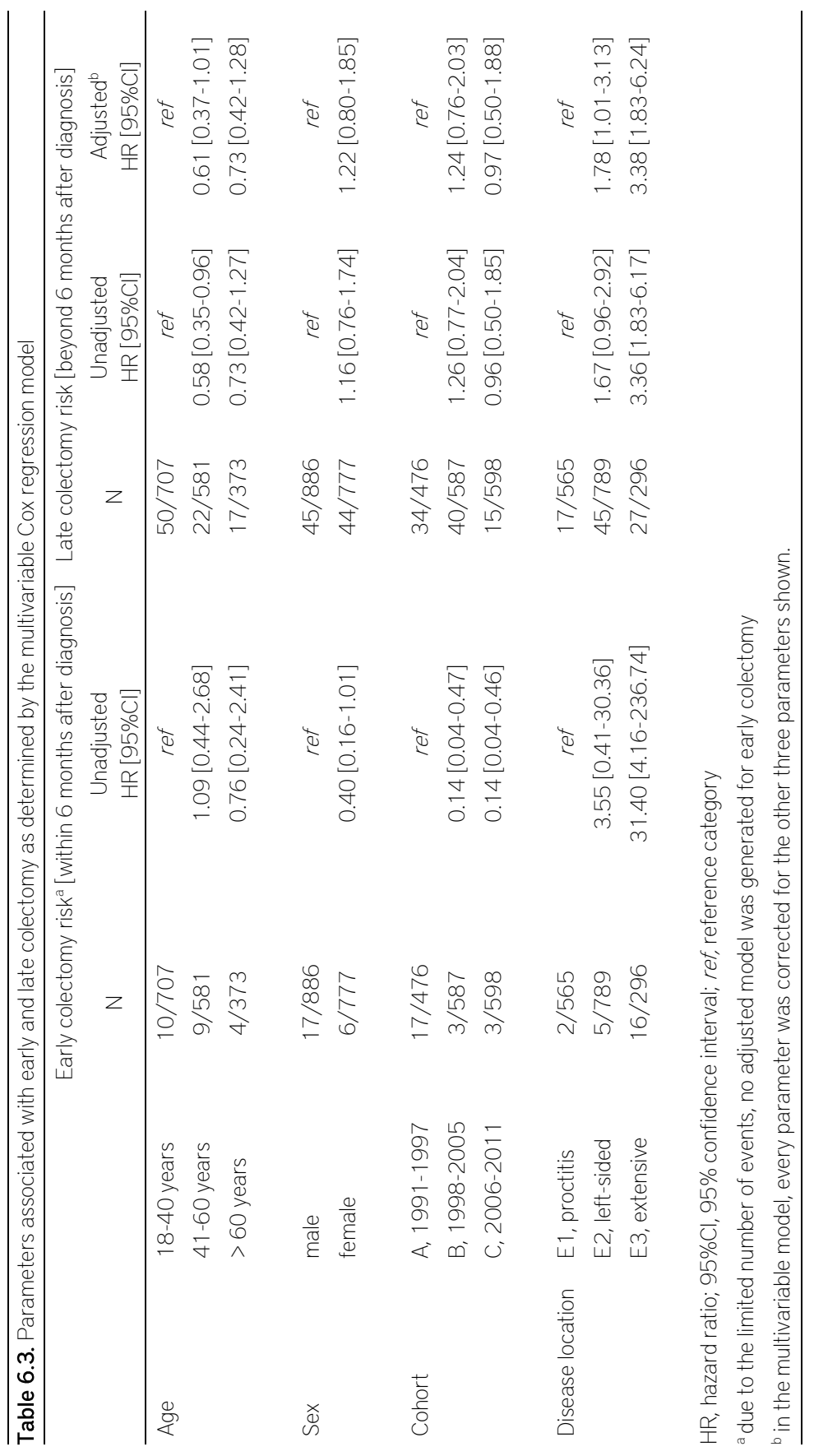


In total, 23 patients underwent early colectomy, 17 patients from the first, 3 from the second, and 3 from the third cohort. Over time, a decrease in the number of early colectomies was observed. The early colectomy rate attenuated from $1.5 \%$ in the first to $0.5 \%$ in the second and to $0.3 \%$ in the most recent cohort. In comparison with cohort A, a 7.2 -fold decrease in early colectomy risk was observed in cohort B [adjusted HR 0.14; 95\% Cl 0.04-0.47] and a 7.4-fold decrease in cohort C [adjusted HR 0.14; 95\% Cl 0.04-0.46]. No difference was found between the two more recent cohorts [adjusted HR 0.98; 95\% Cl 0.20-4.85].

In the group of patients that did not undergo surgery within 6 months, the 5 -year colectomy risk was $4.0 \%$ in cohort A, 5.2\% in cohort B, and 3.6\% in cohort C. No statistically significant change in late colectomy risk was observed between cohorts $[p=0.54]$. The results were similar when the first two cohorts were combined and subsequently compared with cohort $\mathrm{C}[p=0.58]$.

\section{Hospitalization}

In cohort A, 288 UC-related hospitalizations took place in 155 patients, at a median of one admission per patient [range 1-12]. In cohort B, 146 patients were ever hospitalized and counted for a total of 258 hospitalizations [median of one admission per patient, range 1-6]. In the most recent cohort, 129 hospitalizations occurred in 84 patients, with a median of one admission per patient [range 1-6].

\section{Cumulative risk of hospitalization}

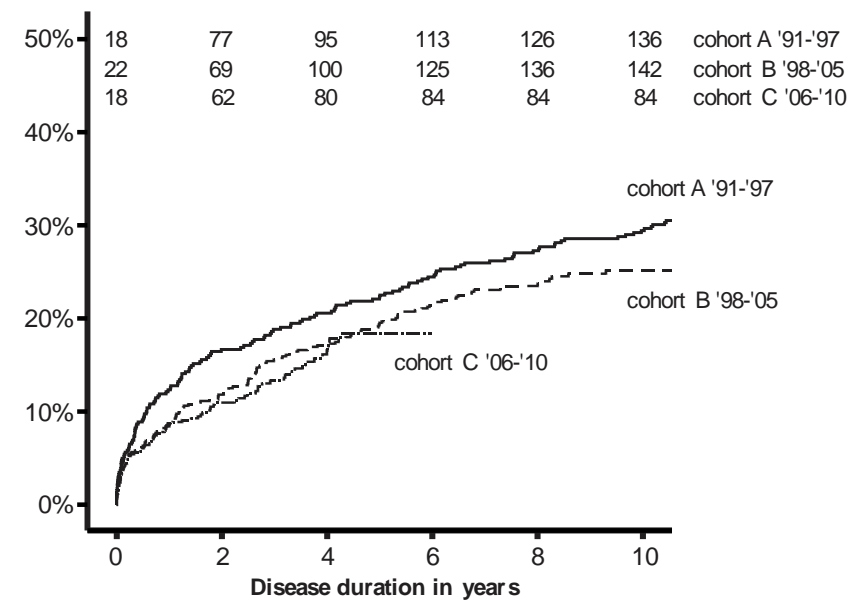

$\begin{array}{lllcccc}\text { No at risk 476 } & 385 & 367 & 349 & 336 & 326 & \text { cohort A '91-'97 } \\ \text { No at risk 587 } & 515 & 482 & 456 & 308 & 191 & \text { cohort B '98-'05 } \\ \text { No at risk 598 } & 392 & 197 & 0 & 0 & 0 & \text { cohort C '06-'10 }\end{array}$

Figure 6.3. The cumulative risk of hospitalization in ulcerative colitis patients among the three cohorts. $\mathrm{N}$, number of patients who required a hospitalization. 
The cumulative risk of first hospitalization after a time span of 1,2, and 5 years was $11.9 \%$, $16.7 \%$, and $22.3 \%$ in cohort $\mathrm{A}, 8.6 \%, 11.8 \%$, and $19.5 \%$ in cohort $\mathrm{B}$, and $8.7 \%, 11.0 \%$, and $18.3 \%$ in cohort C, respectively [see also Figure 6.3]. Compared with the first cohort, no statistical differences between hospitalization risks were observed: adjusted $\mathrm{HR} 0.82 ; 95 \% \mathrm{Cl}$ 0.65-1.03 [cohort B] and adjusted HR 0.74; 95\% Cl 0.56-1.01 [cohort C] [Table 6.4]. Nor were differences observed between the second and third cohort [adjusted HR 0.96; 95\% Cl $0.72-1.28]$. The median number of days per hospital admission decreased over time, from 17 days [IQR 12-26] in cohort A, to 15 days [IQR 10-24] in cohort B, to 13 days [IQR 819] in cohort $C, p<0.01$.

The cumulative 5 -year probability of rehospitalization during disease course was $37.2 \%$ in the first, $48.6 \%$ in the second, and $44.8 \%$ in the most recent cohort. In contrast to the first cohort, rehospitalization was more likely to occur in the second [adjusted HR 1.55; $95 \% \mathrm{Cl} 1.09-2.22]$, whereas no statistically significant effect was observed in the third cohort [adjusted HR 1.11; 95\% Cl 0.69-1.81]. 


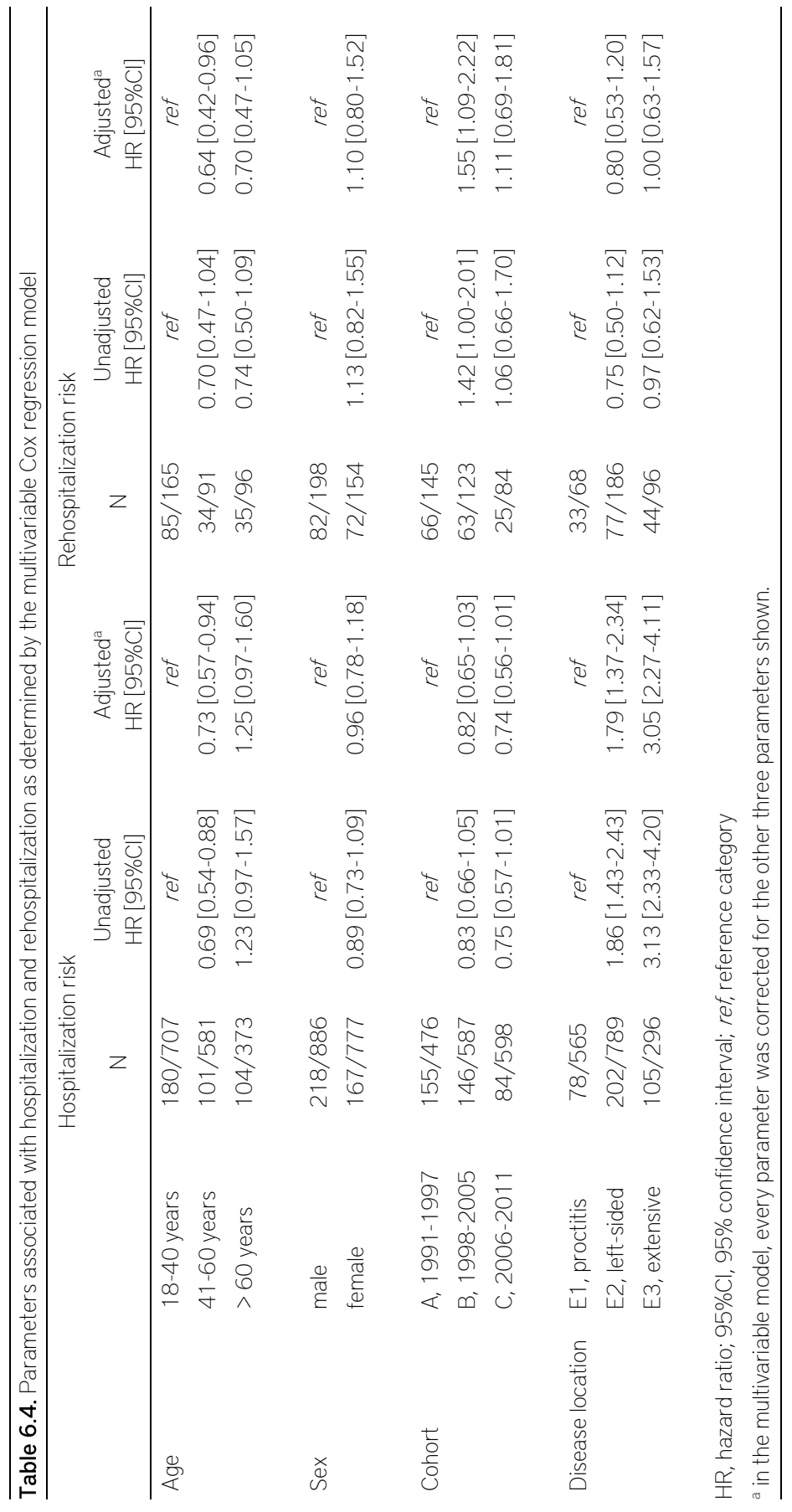




\section{Discussion}

In the Dutch South Limburg area, the risk of early colectomy has decreased over time, albeit no further risk reduction was observed in the most recent cohort diagnosed until 2010. Late colectomy rate was found to be stable over time, as was the hospitalization rate. Duration of hospital admission gradually decreased over time.

This is the first study to assess UC disease outcome in the era of current treatment strategies, including availability of biologicals, in direct comparison with previous eras in the same source population. We studied the time trend in disease outcome since the early $90 \mathrm{~s}$ and could reflect on the clinically relevant questions regarding the effectiveness of treatment changes in UC management at population level. The colectomy rate observed in cohort A [4.1\% after 1 year, $7.5 \%$ after 5 years] was comparable to the rate observed in other population-based studies from the 90s, such as the Scandinavian IBSEN cohort [3.5\% and 7.6\%], the Canadian UMIBDED cohort [3.6\% and 7.6\%], the French EPIMAD cohort [4.0\% and 8.0\%], and the European EC-IBD cohort [4.7\% after 2 years]. ${ }^{3,7,11,37}$

The present study observed a decline in colectomy rate between patients diagnosed between 1991 and 1997 and patients diagnosed between 1998 and 2005. Current literature is inconsistent with respect to the question whether the colectomy rate has changed over time. A recent study in the UMIBDED cohort has shown that late colectomy rate [> 90 days after diagnosis] was 47\% lower in UC patients diagnosed between 2002 and 2008, compared with patients diagnosed between 1987 and 1991. The declining colectomy rate was suggested to be the result of an increasing adoption of immunomodulators in more recent UC cohorts. ${ }^{11}$ Conversely, in Olmsted County, Minnesota, a higher 5-year cumulative colectomy rate was observed in patients diagnosed between 2000 and 2004 compared with patients diagnosed between 1990 and 1999 [24.2\% vs 13.1\%, respectively]. The obvious increase was explained as being the result of an increase in the incidence of refractory Clostridium difficile associated disease. ${ }^{38}$ The colectomy rate in the area of Veszprem, Hungary, was reported to be stable over time. ${ }^{39,40}$ Some time trend studies observed a decrease in the annual colectomy rate over time, but did not take the era of patients' diagnosis and disease course into account. ${ }^{12,13,22}$ These contradicting conclusions indicate that time trends in colectomy rate differ between UC populations, illustrating the importance of studying time trends in disease outcome in the same source population. Area-specific factors, such as microbial superinfections, treatment availability, adoption of treatment strategies, and attitude towards surgery, may contribute to the differences between populations.

The decline in colectomy rate observed in the present study was mainly driven by a decrease in the risk of colectomy within 6 months after diagnosis [early colectomy]. As the colectomy rate had already decreased shortly after diagnosis, the reasons for this decline 
might reside in an improved diagnostic process, i.e. increased awareness of the disease among patients and general practitioners, resulting in a shorter patient or physician delay. Regrettably, data on time between onset of complaints and diagnosis were not available for study. Advances in and availability and application of therapeutic options may also have contributed to the observed decrease in early colectomy risk. In line with this presumption, we documented that immunomodulatory agents were earlier and more frequently used in patients from cohort B and changes herein were already observed within the time window of early colectomy. In previous studies, it has been hypothesized that changes in the timing of, and indication for, immunomodulating therapy played an important role in observed decreasing colectomy rates. ${ }^{11-13}$ A change in the role of surgery in patients with acute, severe disease was probably not causing the decline in colectomy rate, as the frequency of rescue therapy was equal in the first two cohorts. Under the current treatment strategy, the advent of biologicals seemed not to have resulted in a further decrease in early colectomy risk, as the colectomy rate in the biological era [cohort C] was not different from the last cohort of the pre-biological era [cohort B]. Of note, the actual number of patients in cohort $\mathrm{C}$ who received biological treatment within 6 months after diagnosis was low: none of the patients received rescue therapy and only $2.7 \%$ were prescribed IFX as maintenance treatment at that stage.

Late colectomy risk was found to be similar among all three time cohorts. This observation is of interest, acknowledging the changes in the therapeutic armamentarium and treatment strategies in the past two decades. In the present cohort of UC patients, we observed a strong increase in the use of immunomodulators and biological agents. In addition, a decrease in the time to initiation of these treatment options was observed, indicative of a change in treatment strategy. Data on the long-term disease outcome of UC patients on immunomodulator therapy are lacking. Follow-up data from the ACT trials showed a lower colectomy rate in UC patients on IFX therapy compared with patients on placebo after 54 weeks $\left[10 \%\right.$ vs $17 \%$, respectively]. ${ }^{21}$ Although in line with the available guidelines ${ }^{33-34}$ and other population-based cohorts ${ }^{6,13}$, the number of UC patients on immunomodulator and antiTNF $\alpha$ therapy is rather low, even in the most recent cohort [21.7\% and $10.6 \%$, respectively]. Whether a more common use of immunomodulators or anti-TNF $\alpha$ agents would result in a lower late colectomy rate is a very relevant question, but cannot be answered by our real-life, observational data. Moreover, the follow-up of patients in cohort $\mathrm{C}$ was considerably shorter [median 3.3 years] than that of cohort A [median 17.5 years] and cohort B [median 9.5 years], because IFX was registered for UC only in 2006. Although previous data from the EC-IBD study showed that the majority of colectomies are performed within 2 years after diagnosis ${ }^{7}$, future studies with a longer follow-up of patients in the biological era should further reflect the advent of anti-TNF $\alpha$ availability on the long-term surgery rate in UC. Ultimately, our 
data suggest that the changes in UC management have not resulted in a lower late colectomy rate, at least not under the currently recommended treatment strategy.

The hospitalization rate in the 90s of the present study is comparable to the one observed in the Olmsted County. Samuel et al. found a cumulative probability of UC-related hospitalization of $28.6 \%$ after 5 years $^{38}$, in comparison with the $22.3 \%$ [cohort A] observed in the present study. However, a difference in the time trend was observed between studies, as Samuel et al. observed an increase to $44.2 \%$ in more recently diagnosed patients, whereas we observed a decline to $18.3 \%$ in the most recent cohort. This disparity may be explained by a difference in Clostridium difficile-related hospitalization as this was suggested to have contributed to the strong rise in hospitalization rate in the Olmsted County. In the Netherlands, the prevalence of Clostridium difficile is low and not a common trigger for IBD exacerbations. ${ }^{41}$

The main limitation of our study is its observational design. As a result, we cannot assess a causal relationship between the decreased risk of early colectomy and changes in early UC management, such as the early introduction of immunomodulatory agents. In general, in retrospective studies the effects of gradually adopted changes in disease management, such as the implementation of guidelines, or increased disease awareness, and also the gradual adoption of immunomodulators, cannot be assessed accurately. Furthermore, information regarding smoking status and duration of corticosteroid use was not available. Smoking is associated with a better long-term prognosis of $\mathrm{UC}$, and smoking cessation results in a more aggressive disease course thereafter. ${ }^{42-43}$ In general smoking is decreasing in the Netherlands which, if having influence, would have resulted in a more severe disease course over time. Detailed information regarding corticosteroid use would have given insight into the prevalence of steroid-dependent and steroid-refractory disease. Additionally, it is regarded as a marker for average disease course severity, which would have been an interesting outcome parameter in the comparison of the three time cohorts.

Strengths of this study reside in its strict population-based origin, high coverage, and the long period of patient inclusion. The latter offered the opportunity to assess time trends in disease outcome in decades in which marked changes in UC management have taken place. In particular, the inclusion of patients diagnosed after clinical availability of IFX delineated the effect of biological availability on disease outcome. In addition, population-based cohort studies have external validity as they are the best available instrument to study the impact of new therapies in a real-life setting of unselected patients at population level. ${ }^{44,45}$

In conclusion, in the Dutch population-based IBDSL cohort, a decline in early colectomy rate was observed over the past two decades, although no further reduction was observed in the most recent era. Late colectomy rate and hospitalization rate remained unchanged over time, although duration of hospital stay reduced. These results provide an update on the prognosis of UC patients diagnosed nowadays. 


\section{References}

1. Cosnes J, Gower-Rousseau C, Seksik P Cortot A. Epidemiology and natural history of inflammatory bowel diseases. Gastroenterology 2011;140:1785-94.

2. Langholz E, Munkholm P, Davidsen M, Binder V. Course of ulcerative colitis: analysis of changes in disease activity over years. Gastroenterology 1994;107:3-11.

3. Solberg IC, Lygren I, Jahnsen J, et al. Clinical course during the first 10 years of ulcerative colitis: results from a population-based inception cohort [IBSEN Study]. Scand J Gastroenterol 2009;44:431-40.

4. Hoie $\mathrm{O}$, Wolters $F$, Riis $\mathrm{L}$, et al. Ulcerative colitis: patient characteristics may predict 10-yr disease recurrence in a European-wide population-based cohort. Am J Gastroentero/ 2007; 102:1692-

701.

5. Romberg-Camps MJ, Dagnelie PC, Kester AD, et al. Influence of phenotype at diagnosis and of other potential prognostic factors on the course of inflammatory bowel disease. Am J Gastroentero/2009;104:371-83.

6. Jess $T$, Riis $L$, Vind I, et al. Changes in clinical characteristics, course, and prognosis of inflammatory bowel disease during the last 5 decades: a populationbased study from Copenhagen, Denmark. Inflamm Bowel Dis 2007;13:481-9.

7. Hoie $\mathrm{O}$, Wolters $F \mathrm{~L}$, Riis $\mathrm{L}$, et al. Low colectomy rates in ulcerative colitis in an unselected European cohort followed for 10 years. Gastroenterology 2007;132:507-15.

8. Kaplan GG, McCarthy EP, Ayanian JZ, et al. Impact of hospital volume on postoperative morbidity and mortality following a colectomy for ulcerative colitis. Gastroenterology 2008;134:680-7.

9. Lichtenstein GR, Cohen R, Yamashita B, Diamond RH. Quality of life after proctocolectomy with ileoanal anastomosis for patients with ulcerative colitis. J Clin Gastroenterol 2006;40:669-77.

10. Bach SP, Mortensen NJ. Revolution and evolution: 30 years of ileoanal pouch surgery. Inflamm Bowel Dis 2006;12:131-45.

11. Targownik LE, Singh $H$, Nugent $Z$, Bernstein $\mathrm{CN}$. The epidemiology of colectomy in ulcerative colitis: results from a population-based cohort. Am J Gastroenterol2012;107:1228-35.

12. Herrinton LJ, Liu L, Fireman B, et al. Time trends in therapies and outcomes for adult inflammatory bowel disease, Northern California, 1998-2005. Gastroenterology 2009;137:502-11.

13. Kaplan GG, Seow CH, Ghosh S, et al. Decreasing colectomy rates for ulcerative colitis: a population-based time trend study. Am J Gastroenterol 2012;107:1879-87.

14. Rosenberg JL, Wall AJ, Levin B, et al. A controlled trial of azathioprine in the management of chronic ulcerative colitis. Gastroenterology 1975;69:96-9.

15. Kirk AP, Lennard-Jones JE. Controlled trial of azathioprine in chronic ulcerative colitis. British medical journal 1982;284:1291-2.

16. Ardizzone S, Molteni P, Imbesi V, et al. Azathioprine in steroid-resistant and steroid-dependent ulcerative colitis. $J$ Clin Gastroenterol 1997;25:330-3.

17. Fraser AG, Orchard TR, Jewell DP. The efficacy of azathioprine for the treatment of inflammatory bowel disease: a 30 year review. Gut 2002;50:485-9.

18. Rutgeerts P, Sandborn WJ, Feagan BG, et al. Infliximab for induction and maintenance therapy for ulcerative colitis. N Eng/ J Med 2005;353:246276.

19. Reinisch W, Sandborn WJ, Rutgeerts P, et al. Long-term infliximab maintenance 
therapy for ulcerative colitis: the ACT-1 and -2 extension studies. Inflamm Bowel Dis 2012;18:201-11.

20. Sandborn WJ, van Assche G, Reinisch W, et al. Adalimumab induces and maintains clinical remission in patients with moderate-to-severe ulcerative colitis. Gastroenterology 2012;142:257-65 e1-3.

21. Sandborn WJ, Rutgeerts P, Feagan BG, et al. Colectomy rate comparison after treatment of ulcerative colitis with placebo or infliximab. Gastroenterology 2009; 137:1250-60; quiz 520.

22. Reich KM, Chang HJ, Rezaie A, et al. The incidence rate of colectomy for medically refractory ulcerative colitis has declined in parallel with increasing anti-TNF use: a time-trend study. Aliment Pharmacol Ther 2014, 10.1111/apt.12873.

23. Chatu S, Saxena S, Subramanian V, et al. The impact of timing and duration of thiopurine treatment on first intestinal resection in Crohn's disease: national UK population-based study 1989-2010. Am J Gastroentero/ 2014;109:409-16.

24. Picco MF, Zubiaurre I, Adluni M, et al. Immunomodulators are associated with a lower risk of first surgery among patients with non-penetrating nonstricturing Crohn's disease. Am J Gastroentero/2009;104:2754-9.

25. van den Heuvel TR, Jonkers DM, Jeuring SF, et al. Cohort Profile: The Inflammatory Bowel Disease South Limburg Cohort [IBDSL]. Int J Epidemiol 2015, DOl 10.1093/ije/dyv088.

26. Russel MG, Dorant E, Volovics A, et al. High incidence of inflammatory bowel disease in The Netherlands: results of a prospective study. The South Limburg IBD Study Group. Dis Colon Rectum 1998:41:33-40.

27. Romberg-Camps MJ, Hesselink-van de Kruijs MA, Schouten LJ, et al. Inflammatory Bowel Disease in South Limburg [the Netherlands] 1991-2002: Incidence, diagnostic delay, and seasonal variations in onset of symptoms. J Crohns Colitis 2009:3:115-24.
28. Statistiek CBv. Statline. http://statline.cbs.nl/, 01-02-2015.

29. Oostenbrink JB, Rutten FF. Cost assessment and price setting of inpatient care in The Netherlands. the DBC casemix system. Health care management science 2006;9:287-94.

30. Lennard-Jones JE. Classification of inflammatory bowel disease. Scand J Gastroentero/ Supp/ 1989;170:2-6; discussion 16-9.

31. Satsangi J, Silverberg MS, Vermeire S, Colombel JF. The Montreal classification of inflammatory bowel disease: controversies, consensus, and implications. Gut 2006;55:749-53.

32. Travis SP, Stange EF, Lemann M, et al. European evidence-based Consensus on the management of ulcerative colitis: Current management. J Crohns Colitis 2008:2:24-62.

33. Dignass A, Lindsay JO, Sturm A, et al. Second European evidence-based consensus on the diagnosis and management of ulcerative colitis part 2 : current management. J Crohns Colitis 2012;6:991-1030

34. van Bodegraven AA, van Everdingen JJ, Dijkstra G, et al. [Guideline 'Diagnosis and treatment of inflammatory bowel disease in adults'. I. Diagnosis and treatment]. Nederlands tijdschrift voor geneeskunde 2010;154:A1899.

35. Dignass A, Eliakim R, Magro F, et al. Second European evidence-based consensus on the diagnosis and management of ulcerative colitis part 1 : definitions and diagnosis. J Crohns Colitis 2012;6:965-90.

36. Kleinbaum D.G. KM. Survival Analysis, A Self-Learning Text. New York: Springer; 2005.

37. Charpentier C, Salleron J, Savoye G, et al. Natural history of elderly-onset inflammatory bowel disease: a population-based cohort study. Gut 2014;63:423-32.

38. Samuel S, Ingle SB, Dhillon S, et al. Cumulative Incidence and Risk Factors for Hospitalization and Surgery in a 
Population-based Cohort of Ulcerative Colitis. Inflamm Bowel Dis 2013, 10.1097/MIB.0b013e31828c84c5.

39. Lakatos PL, David G, Pandur T, et al. IBD in the elderly population: results from a population-based study in Western Hungary, 1977-2008. J Crohns Colitis 2011;5:5-13.

40. Lakatos L, Kiss LS, David G, et al. Incidence, disease phenotype at diagnosis, and early disease course in inflammatory bowel diseases in Western Hungary, 2002-2006. Inflamm Bowel Dis 2011;17:2558-65.

41. Masclee GM, Penders J, Jonkers DM, et al. Is clostridium difficile associated with relapse of inflammatory bowel disease? results from a retrospective and prospective cohort study in the Netherlands. Inflamm Bowel Dis 2013;19:2125-31

42. Mokbel M, Carbonnel F, Beaugerie L, Gendre JP, Cosnes J. [Effect of smoking on the long-term course of ulcerative colitis]. Gastroenterol Clin Biol 1998:22:858-62.

43. Beaugerie L, Massot N, Carbonnel F, et al. Impact of cessation of smoking on the course of ulcerative colitis. Am J Gastroentero/2001;96:2113-6.

44. Gower-Rousseau C, Savoye G, Colombel $J F$, Peyrin-Biroulet L. Are we improving disease outcomes in IBD? A view from the epidemiology side. Gut 2013, 10.1136/gutjnl-2013-306045.

45. van den Heuvel T, Jonkers D, Jeuring S, et al. DOP019 The relevance of population based IBD biobanks: a metaanalysis and introduction of the IBD-SL biobank cohort. J Crohns Colitis 2014;8:S23 



\section{CHAPTER 7}

\section{Corticosteroid sparing in inflammatory bowel disease is more often achieved in the immunomodulator and biological era}

Steven FG Jeuring, Vince BC Biemans, Tim RA van den Heuvel, Maurice P Zeegers, Wim H Hameeteman, Marielle JL Romberg-Camps, Liekele E Oostenbrug, Ad AM Masclee, Daisy MAE Jonkers, Marieke J Pierik

Am J Gastroenterol. 2018 Mar; 113(3):384-395 


\section{Abstract}

\section{Background}

Corticosteroid-free remission is an emerging treatment goal in the management of inflammatory bowel disease. In the population-based IBDSL cohort, we studied temporal changes in corticosteroid use and assessed the corticosteroid sparing effects of immunomodulators and biologicals in real-life.

\section{Methods}

In total, 2823 newly-diagnosed patients with Crohn's disease [CD] and ulcerative colitis [UC] were included. Corticosteroid exposure and cumulative days of use were compared between patients diagnosed in 1991-1998 [CD: $n=316, U C: n=539], 1999-2005$ [CD: $n=387$, UC: $n=527$ ], and 2006-2011 [CD: $n=459, \cup C: n=595]$. Second, the corticosteroid sparing effects of immunomodulators and biologicals were assessed.

\section{Results}

Over time, the corticosteroid exposure rate was stable [54.0\% in CD and 31.4\% in UC], even as the cumulative corticosteroid use in the first disease year [CD: 83 days [IQR 35-189], UC: 62 days [IQR 0-137]]. On the long-term, a gradual decrease was seen in CD [era '91-'98: 366 days [IQR 107-841], era '06-'11: 120 days [IQR 72-211], $p<0.01$ ], while in UC an initial decrease was observed (era '91-'98: 184 days (IQR 86-443), era '99-'05: 166 days [IQR 74-281], $p=0.03]$, and stabilization thereafter. Immunomodulator and biological users had a lower risk of requiring corticosteroids than matched controls in CD only [33.6\% vs. $49.9 \%, p<0.01$ and $25.7 \%$ vs. $38.2 \%, p=0.04$, respectively].

\section{Conclusion}

In a real-world setting, more-recently diagnosed IBD patients used lower amounts of corticosteroids as of the second year of disease. For CD, a significant association was found with the use of immunomodulators and biologicals. These conclusions support the increasing use of these treatment modalities. 


\section{Introduction}

The primary treatment goal in the management of Crohn's disease [CD] and ulcerative colitis [UC] is the induction and maintenance of clinical remission, according to international guidelines. ${ }^{1,2}$ For decades, corticosteroids have been a cornerstone in achieving this treatment goal, given the high response [80\%] and remission [73\%] rates. ${ }^{3-7}$ Despite their high efficacy for remission induction, corticosteroids are not recommended as maintenance therapy ${ }^{8,9}$, because of a low efficacy on the long-term and severe side effects. Typical short-term effects include hyperglycemia and Cushingoid features, while on the long-term patients have a higher risk of osteoporosis, cardiovascular events, and glaucoma. ${ }^{10-12}$ Furthermore, corticosteroid use increases the risk of opportunistic infections and death, especially in elderly patients. ${ }^{13-15}$ Therefore, corticosteroid sparing is an emerging goal in IBD management, as advocated in the latest European and American guidelines. ${ }^{1,2,16}$

Randomized controlled trials have shown that the immunomodulatory agents azathioprine and 6-mercaptopurine are superior to placebo for the maintenance of remission and have a corticosteroid sparing effect in both $C D^{17}$ and $U C^{18}$. The efficacy of anti-tumor necrosis factor $\alpha$ [anti-TNF $\alpha$ ] agents to induce and maintain disease remission and to reduce the need for corticosteroid therapy has been established in trial populations as well, both in $C^{19,20}$ and $U C^{21}$. It is unknown whether these drugs also have corticosteroid-sparing effects in real-life. Such information is important, because the real-life situation often differs from a trial setting, for example in terms of a more heterogeneous patient population with more comorbidities, less stringent disease monitoring, and sometimes treatment indication, due to local drug availability and costs. ${ }^{22}$ Previous observational studies demonstrated that both immunomodulators and anti-TNF $\alpha$ agents are increasingly used in the medical management of IBD. ${ }^{23-25}$ Albeit many studies have discussed the impact of changing treatment strategies on the phenotype progression and surgery rate ${ }^{23-29}$, little is known about their impact on the total amount of corticosteroids IBD patients use during disease course.

The primary aim of this study was therefore to study the evolution of corticosteroid use over the past two decades in a large population-based cohort of IBD patients. Second, the real-life corticosteroid sparing effects of immunomodulators and anti-TNFa agents were studied. 


\section{Methods}

\section{Study population}

The Inflammatory Bowel Disease South Limburg [IBDSL] cohort is a long-term, populationbased, prospective inception cohort of patients with IBD. The design and rationale of the IBDSL cohort are described in detail elsewhere..$^{30}$ In brief, newly-diagnosed adult IBD patients in the area of South Limburg, the Netherlands, are being registered and followed as from 1991. Via a multi-faceted identification strategy, 1162 CD and 1661 UC patients have been included and were followed, which was estimated to be over 93\% of the IBD population in South Limburg. ${ }^{30}$ Patients were diagnosed between January 1st 1991 and December 31st 2010 [UC] and July 1st 2011 [CD] and followed until the end of current data collection [2011 for UC, 2014 for CD]. The IBDSL project has been approved by the Medial Ethics Committee of the Maastricht University Medical Center [NL31636.068.10], is registered at www.ClinicalTrial.gov [NCT02130349], and follows the revised declaration of Helsinki.

\section{Data collection}

All data were retrieved via a thorough review of each patient's individual record, thereby assuring case ascertainment. Demographic data, disease phenotype [classified according to the Montreal consensus ${ }^{31-32}$, and medication data were collected by trained physicians, using standardized registration forms.

For the purpose of the present study, all patient visits were scrutinized for information on corticosteroid use. Corticosteroid use was defined as the use of a systemic corticosteroid, either orally or intravenously administered. Budesonide use was addressed separately in this study. Local application of corticosteroids, including enemas or suppositories containing prednisone, budesonide, or dexamethasone were not studied, because of their low systemic availability. For every visit, corticosteroid use, dose, and administration route were recorded, including reasons for treatment initiation and cessation, and the tapering regimen. In case no regimen was specified, a standard tapering regimen of $5 \mathrm{mg}$ per week was assumed as from the date of last visit, in line with the Dutch IBD guideline. ${ }^{33}$ Immunomodulators comprised azathioprine, mercaptopurine, tioguanine, and methotrexate. Anti-TNF $\alpha$ agents registered during the studied period in the Netherlands were infliximab and adalimumab.

\section{Study endpoints and definitions}

The primary endpoints of the study were: [l] the corticosteroid exposure [measured as the cumulative probability of ever requiring corticosteroids and as the point prevalence of corticosteroid use during disease course] and [II] the cumulative days of corticosteroid use. The latter was defined as the total number of calendar days a patient has been treated with corticosteroids [ $\geq 5 \mathrm{mg} /$ day]. 
The temporal changes in the primary endpoints were assessed by comparing the outcome measures between three subsequent eras: era 1991-1998, era 1999-2005, and era 2006-2011. Eras were distinguished by the years of anti-TNF $\alpha$ availability in the Netherlands [1999 for CD, 2006 for UC] and comprised all IBD patients that were diagnosed within that time span. Analyses were performed separately for CD and UC.

The secondary aim of the study was to assess the corticosteroid sparing effect of immunomodulators and anti-TNF $\alpha$ agents, measured by the difference in the need for a course of corticosteroids between users and non-users. Corticosteroid sparing was addressed in terms of the risk of requiring a new course of corticosteroids after the specific treatment was started.

\section{Statistical analyses}

Continuous variables were presented as means with standard deviations [SD] or as medians with interquartile ranges [IQR], depending on normality of the underlying distribution. Variables were subsequently compared between eras by using one-way ANOVA or Kruskal-Wallis tests, respectively. Categorical variables were presented as numbers with percentages and compared between eras by using Chi-square tests.

Corticosteroid exposure was assessed by estimating the cumulative probability of ever requiring corticosteroids during disease course, using Kaplan-Meier survival statistics. Differences between eras were assessed by using multivariable Cox regression modeling, adjusting for differences in age at diagnosis, sex, and initial disease phenotype between eras. Differences in hazards between eras were expressed as adjusted hazard ratios [aHR] with the $95 \%$ confidence intervals $[95 \% \mathrm{Cl}$ ] or by the accompanying [adjusted] $p$-value from the multivariable model.

The point prevalence of corticosteroid use was determined by calculating the proportion of patients that were on corticosteroid therapy at the end of every week of followup. The peak prevalence was defined as the time point at which most IBD patients were on corticosteroid therapy, and differences in peak heights between eras were assessed by using a Chi-square test. Additionally, the average corticosteroid point-prevalence was calculated and compared between eras by performing linear regression modeling on log-transformed prevalences, adjusting for the same confounders as previously described.

Cumulative days of corticosteroid use were determined in corticosteroid users with a minimum follow-up of five years in order to avoid extrapolation of the data, particularly in more recent eras with a shorter mean follow-up time. Extrapolation could lead to an overestimation of the cumulative days of corticosteroid use, because the probability of requiring corticosteroids is highest in the early years of disease. ${ }^{34}$ The cumulative days of corticosteroid use in the first year and first five years of disease were calculated in these 
patients and subsequently compared between eras by using linear regression modeling on log transformed values, adjusting for the previously discussed confounders.

The corticosteroid sparing effect of immunomodulators and anti-TNF $\alpha$ agents was studied by using propensity score matching analyses. CD and UC patients with a first immunomodulator or anti-TNFa prescription within 12 months after diagnosis [users] were matched to patients that had no prescription within that time window [nonusers]. Matching was based on the propensity score derived from a logistic regression model with immunomodulator use or anti-TNF $\alpha$ use as the dependent variable, respectively [nearest neighbor 1:1 matching without replacement, caliper 0.2]. Parameters included in the propensity model were age at diagnosis, sex, and early disease course characteristics [i.e. disease phenotype, hospitalization, and corticosteroid use within three months after diagnosis]. By balancing these characteristics between users and non-users, we aimed to control for measured confounding that results from treatment allocation. After matching, nonusers and users were followed as from the same point in disease course, by assigning nonusers an 'index date', based on the date at which the matched user starts with the specific therapy. As from this point, patients were followed until the day on which corticosteroid therapy was started, immunomodulator or anti-TNFa therapy was stopped, or end of followup was reached. Kaplan-Meier survival analyses were used to estimate the cumulative probability of requiring corticosteroids. Cox regression models were used to assess differences in outcomes between users and non-users. Analyses were further adjusted for confounding by adding the cumulative days of corticosteroid use prior to the index date as parameter in the model. As for the outcomes, the first six months after the index date were censored, because many users are on corticosteroid therapy at the start of immunomodulator or anti-TNFa therapy. Furthermore, events that took place within six months after therapy discontinuation were included, because therapy cessation may have preceded an event of interest.

Two-sided $p$-values lower than 0.05 were considered statistically significant. All statistical analyses were performed using IBM SPSS Statistics for Windows, Version 23.0. [IBM Corp. Armonk, NY, USA]. 


\section{Results}

\section{Patient population}

In total, 1162 CD patients and 1661 UC patients were studied. Regarding CD, 316 patients were diagnosed in era 1991-1998 ['91-'98], 387 patients in era 1999-2005 ['99-'05], and 459 in era 2006-2011 ['06-'11]. Regarding UC, 539 patients were diagnosed in the first, 527 patients in the second, and 595 in the third era. Patients were followed for a median of 7.5 years [IQR 4.1-12.4] [CD] and 8.5 years [IQR 4.4-14.7] [UC]. Detailed patient characteristics are shown in Table 7.1 [CD] and Table 7.2 [UC].

Table 7.1. Patient characteristics of Crohn's disease patients from the IBDSL cohort

\begin{tabular}{|c|c|c|c|c|c|}
\hline & & $\begin{array}{l}\text { era } 1991-1998 \\
\quad[N=316]\end{array}$ & $\begin{array}{l}\text { era } 1999-2005 \\
\quad[N=387]\end{array}$ & $\begin{array}{c}\text { era } 2006-2011 \\
\quad[N=459]\end{array}$ & \\
\hline Age in years ${ }^{a}$ & mean $[\mathrm{SD}]$ & $36.2[15.8]$ & $37.5[15.8]$ & $38.8[16.1]$ & $p=0.08$ \\
\hline Male & $N[\%]$ & 121 [38.3] & $137[35.4]$ & $176[38.3]$ & $p=0.62$ \\
\hline Current smoker ${ }^{\mathrm{a}, \mathrm{b}}$ & $N[\%]$ & $141[55.3]$ & $178[49.4]$ & $175[46.3]$ & $p=0.08$ \\
\hline $\begin{array}{l}\text { Disease duration } \\
\text { in years }\end{array}$ & $\begin{array}{l}\text { median } \\
{[I Q R]}\end{array}$ & 16.1 [10.3-19.3] & 9.7 [8.0-11.8] & $4.2[2.9-5.6]$ & $p<0.01$ \\
\hline Disease location & & & & & $p=0.04$ \\
\hline L1, ileum & $N[\%]$ & 155 [49.1] & 170 [43.9] & 175 [38.1] & \\
\hline L2, colon & $N[\%]$ & 94 [29.7] & 127 [32.8] & $150[32.7]$ & \\
\hline L3, ileocolon & $N[\%]$ & $63[19.9]$ & $80[20.7]$ & $124[27.0]$ & \\
\hline Ł4, only upper GI & $N[\%]$ & $4[1.3]$ & $10[2.6]$ & $10[2.2]$ & \\
\hline Disease behavior ${ }^{c}$ & & & & & $p=0.03$ \\
\hline B1, inflammatory & $N[\%]$ & $232[73.4]$ & 299 [77.3] & $369[80.4]$ & \\
\hline B2, stricturing & $N[\%]$ & $51[16.1]$ & $57[14.7]$ & $69[15.0]$ & \\
\hline B3, penetrating & $N[\%]$ & $33[10.4]$ & $31[8.0]$ & $21[4.6]$ & \\
\hline Perianal disease ${ }^{a}$ & $N[\%]$ & $30[9.5]$ & $27[7.0]$ & $37[8.1]$ & $p=0.48$ \\
\hline Upper Gl locationa & $N[\%]$ & $21[6.6]$ & $43[11.1]$ & $60[13.1]$ & $p=0.02$ \\
\hline $\begin{array}{l}\text { Cumulative exposure } \\
\text { to immunomodulators }\end{array}$ & & & & & $p<0.01$ \\
\hline 1-year exposure & $N[\%]$ & 28 [9.1] & 117 [31.0] & $221[49.5]$ & \\
\hline 5-year exposure & $N[\%]$ & 91 [30.6] & 209 [56.6] & $286[70.8]$ & \\
\hline $\begin{array}{l}\text { Cumulative exposure } \\
\text { to anti-TNF } \alpha \text { agents }\end{array}$ & & & & & $p<0.01$ \\
\hline 1-year exposure & $N[\%]$ & $0[0]$ & $27[7.1]$ & 94 [21.2] & \\
\hline 5-year exposure & $\mathrm{N}[\%]$ & $9[3.1]$ & 73 [19.9] & $161[41.2]$ & \\
\hline
\end{tabular}

$\mathrm{SD}$, standard deviation; IQR, interquartile range; TNF $\alpha$, tumor necrosis factor $\alpha$

a at diagnosis

${ }^{\mathrm{b}}$ smoking status was not available in 169 cases

c disease location according to Montreal consensus 
Table 7.2. Patient characteristics of ulcerative colitis patients from the IBDSL cohort

\begin{tabular}{|c|c|c|c|c|c|}
\hline & & $\begin{array}{l}\text { era } 1991-1998 \\
\quad[N=539]\end{array}$ & $\begin{array}{l}\text { era } 1999-2005 \\
\quad[N=527]\end{array}$ & $\begin{array}{c}\text { era } 2006-2011 \\
{[N=595]}\end{array}$ & \\
\hline Age in years ${ }^{a}$ & mean [SD] & $43.2[15.8]$ & $45.7[16.3]$ & $48.3[17.4]$ & $p<0.01$ \\
\hline Male & $\mathrm{N}[\%]$ & $302[56.0]$ & $288[54.6]$ & 295 [49.6] & $p=0.07$ \\
\hline $\begin{array}{l}\text { Disease duration } \\
\text { in years }\end{array}$ & $\begin{array}{l}\text { median } \\
{[\mathrm{IQR}]}\end{array}$ & $16.9[14.9-19.0]$ & $8.9[7.5-10.9]$ & $3.3[2.0-4.7]$ & $p<0.01$ \\
\hline Disease location ${ }^{\mathrm{a}, \mathrm{c}}$ & & & & & $p<0.01$ \\
\hline E1, proctitis & $N[\%]$ & 156 [29.5] & $177[33.7]$ & $230[38.7]$ & \\
\hline E2, left-sided & $N[\%]$ & $291[55.1]$ & $249[47.4]$ & $249[41.8]$ & \\
\hline E3, extensive & $N[\%]$ & $81[15.3]$ & 99 [18.9] & $116[19.5]$ & \\
\hline \multicolumn{6}{|l|}{ Cumulative exposure } \\
\hline to immunomodulators & & & & & $p<0.01$ \\
\hline 1-year exposure & $N[\%]$ & 13 [2.5] & $47[8.9]$ & $56[9.5]$ & \\
\hline 5-year exposure & $N[\%]$ & $46[8.7]$ & $127[24.2]$ & $102[22.5]$ & \\
\hline $\begin{array}{l}\text { Cumulative exposure } \\
\text { to anti-TNF } \alpha \text { agents }\end{array}$ & & & & & $p<0.01$ \\
\hline 1-year exposure & $N[\%]$ & $0[0]$ & $3[0.6]$ & $26[4.4]$ & \\
\hline 5-year exposure & $\mathrm{N}[\%]$ & $0[0]$ & $25[4.8]$ & $52[10.6]$ & \\
\hline
\end{tabular}

$\mathrm{SD}$, standard deviation; IQR, interquartile range; $\mathrm{TNF} \alpha$, tumor necrosis factor $\alpha$

a at diagnosis

${ }^{\mathrm{b}}$ disease location according to Montreal consensus

${ }^{c}$ disease location could not be retrieved in 13 cases

smoking data were not available for UC patients

Over time, both $C D$ and $U C$ patients were more frequently exposed to immunomodulators and anti-TNF $\alpha$ agents. For immunomodulators, the cumulative 5 -year exposure rates increased from 30.6\% [era '91-'98] to 56.6\% [era '99-'05] to 70.8\% [era '06-'11] in CD [ $p<0.01$ ] and from $8.7 \%$ [era '91-'98] to 24.2\% [era '99-'05] [ $p<0.01$ ] and stabilized thereafter [22.5\%, era '06-'11] [ $p=0.94]$ in UC. For anti-TNF $\alpha$ agents, the cumulative 5 -year exposure rates increased from 3.1\% [era '91-'98] to 19.9\% [era '99-'05] to 41.2\% [era '06-'11] and from 0\% [era '91-'98] to 4.8\% [era '99-'05] to 10.6\% [era '06-'11], respectively [both $p<$ 0.01 ]. Furthermore, both therapies were started earlier in disease course in more recent eras, reflected by increasing cumulative 1-year exposure rates [see Tables 7.1 and 7.2].

\section{Corticosteroid exposure}

In $C D, 665$ patients were ever exposed to corticosteroid therapy, corresponding to a 5 -year exposure rate of $54.0 \%$. The exposures to corticosteroids and budesonide are shown in Figure 7.1 . 


\section{Crohn's disease}

A

Cumulative exposure to prednisone

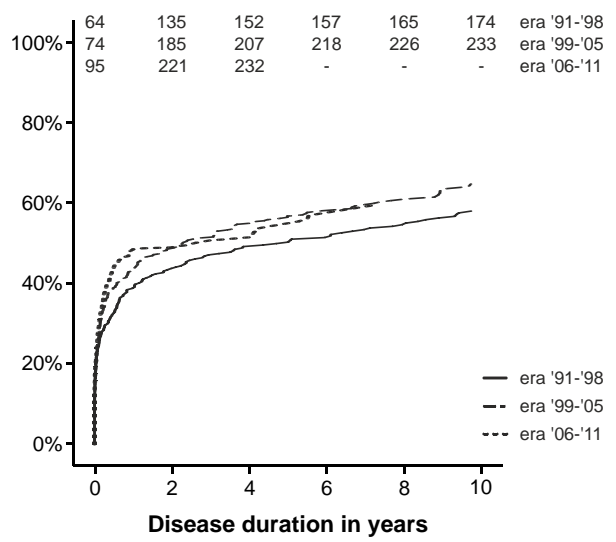

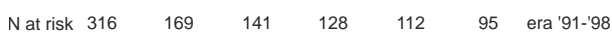

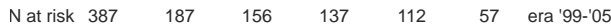

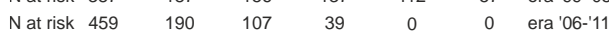

\section{Ulcerative colitis}

C

\section{Cumulative exposure to prednisone}

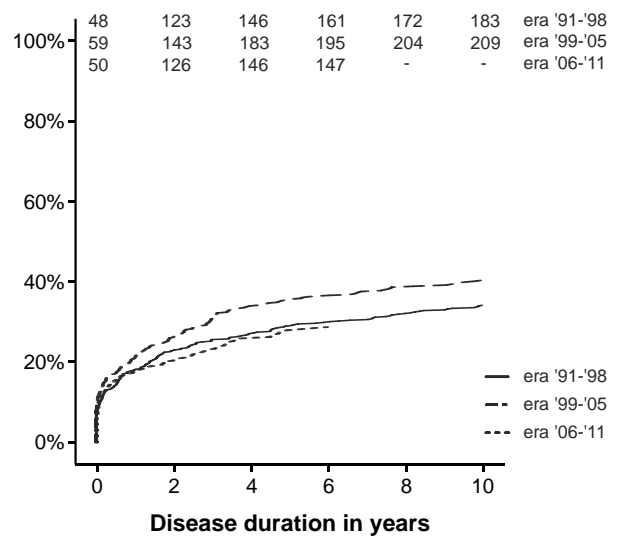

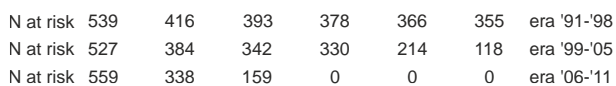

B

Cumulative exposure to budesonide

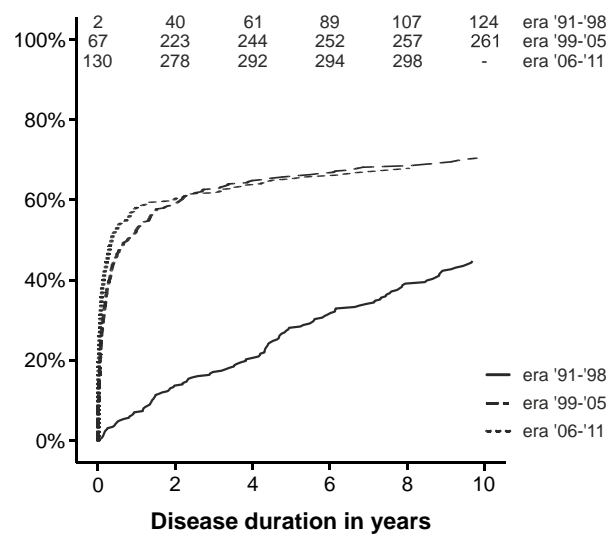

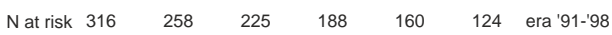

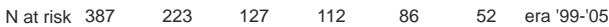

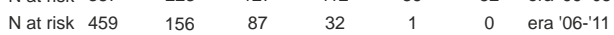

Figure 7.1. The cumulative exposures to corticosteroids and budesonide in Crohn's disease [A and B] and ulcerative colitis [C and D], stratified by era of diagnosis.

$\mathrm{N}$, number of patients who used corticosteroid and budesonide therapy 
Although the 5-year exposure rate was not different between the three eras [50.0\%, 56.2\%, and $54.8 \%$, respectively, adjusted $p=0.20]$, more CD patients were already exposed to corticosteroids in the first year of disease in more recent eras [38.5\% in era ' $91{ }^{\prime}{ }^{\prime} 98,43.2 \%$ in era '99-'05, and $48.5 \%$ in era '06-'11, adjusted $p=0.04$ ]. In parallel, the 5 -year exposure to budesonide increased from $26.8 \%$ in the first to $65.7 \%$ in the second era and remained stable afterwards [66.2\% in the third era], adjusted $p<0.01$. Similar to corticosteroids, the 1-year budesonide exposure rate increased over time: 7.1\% [era 91-98], 51.9\% [era 99-05], and $59.2 \%$ [era 06-11], $p<0.01$.

In UC, 558 patients were exposed to corticosteroid therapy, corresponding to a 5 year exposure rate of $31.4 \%$, significantly lower than the exposure rate in CD [54.0\%], $p<0.01$. The 5-year exposure rate was higher in era '99-'05 [36.3\%] than in eras ' 91 -'$^{\prime} 98$ [28.9\%] and '06-'11 [28.7\%] [ $p=0.03]$, while no difference was observed in the 1 -year exposure rate between eras [18.0\% in era ' 91 -' $98,22.0 \%$ in era ' $99-{ }^{\prime} 05,21.9 \%$ in era '06$111, p=0.24]$. Budesonide use was significantly lower in UC than in CD, but also increased over time, reflected by 5 -year rates of $0.4 \%, 5.3 \%$, and $7.2 \%$ in the three subsequent eras [adjusted $p<0.01$ ].

\section{Corticosteroid point prevalence}

The point prevalence of corticosteroid use during disease course is shown in Figures 7.2 [CD] and 7.3 [UC]. The peak prevalence was higher in CD [26.7\% after week 8] than in UC [11.2\% after week 6], $p<0.01$. Beyond the first year of disease, the mean point prevalence in $C D$ decreased over time from $9.0 \% \pm 3.4 \%$ in era ' 91 -' 98 to $3.9 \% \pm 2.0 \%$ in era ' 99 -' 05 to $1.4 \%$ $\pm 1.0 \%$ in era '06-' 11 , adjusted $p<0.01$. A similar time-trend was observed in UC: $4.2 \% \pm$ $1.0 \%$ in the first to $3.4 \% \pm 1.2 \%$ in the second to $2.1 \% \pm 1.5 \%$ in the third era, adjusted $p<0.01$.

\section{Cumulative days of corticosteroid use}

The cumulative days of corticosteroid use was assessed in IBD patients with a follow-up of at least five years, whom ever had a corticosteroid prescription [ $n=430$ for $C D, n=386$ for $U C$ ]. In CD, we observed that corticosteroid users received a median of 83 days [IQR 35189] of corticosteroids within the first year and 167 days [IQR 78-414] within the first five years after CD diagnosis. Over time, the cumulative corticosteroid use in the first year of disease was stable [median 107 days [IQR 35-268] in era '91-'98, 81 days [IQR 13-170] in era '99-'05, and median 79 days [IQR 54-160] in era '06-'11, $p=0.15$, see Figure 7.4A], whereas the cumulative number of treatment days in the first five years of disease significantly decreased over time $[p<0.01$, see Figure $7.4 \mathrm{~B}$ and Table 7.3A]. 

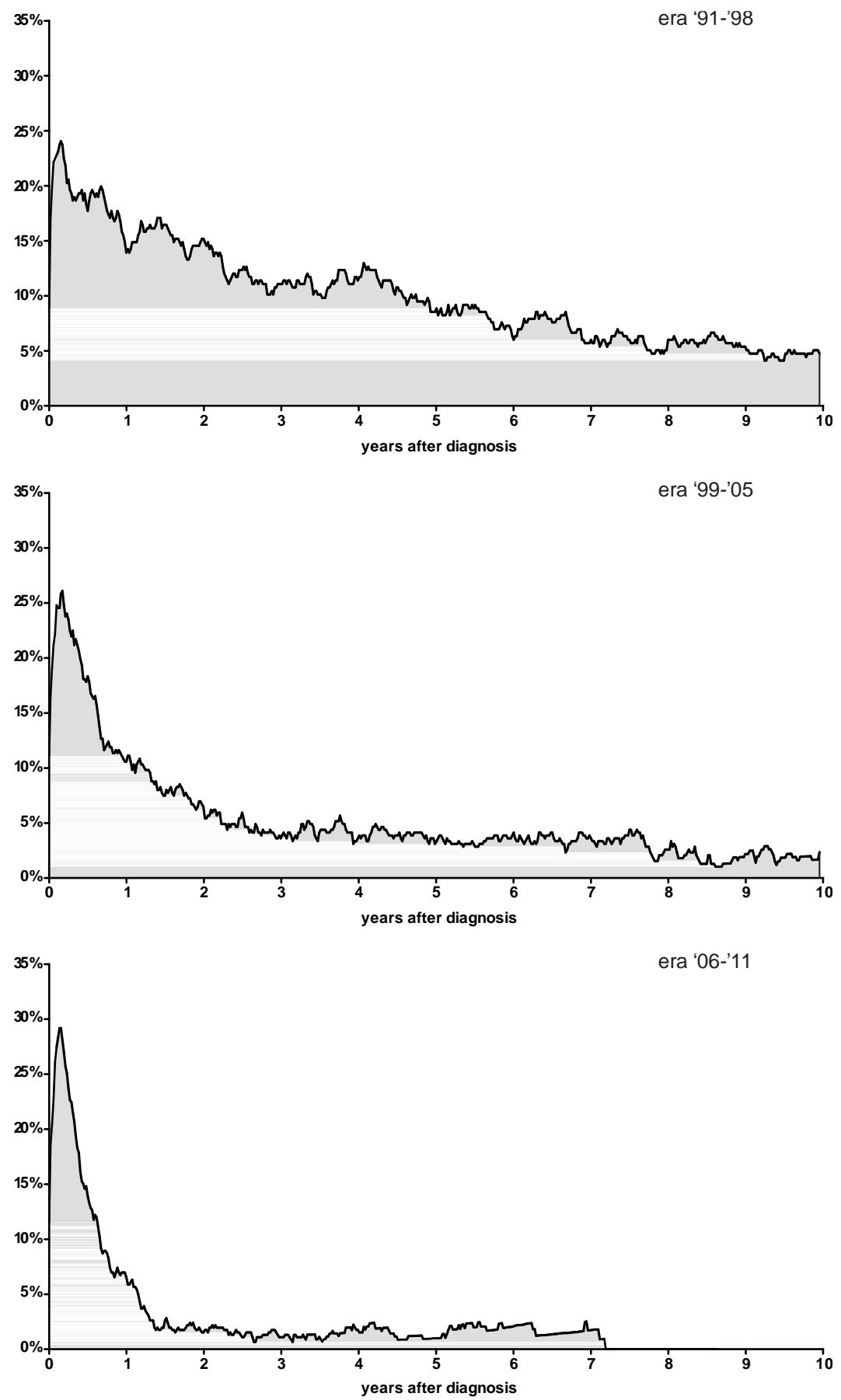

Figure 7.2. Point prevalence of systemic corticosteroid use in Crohn's disease, stratified by era of diagnosis 

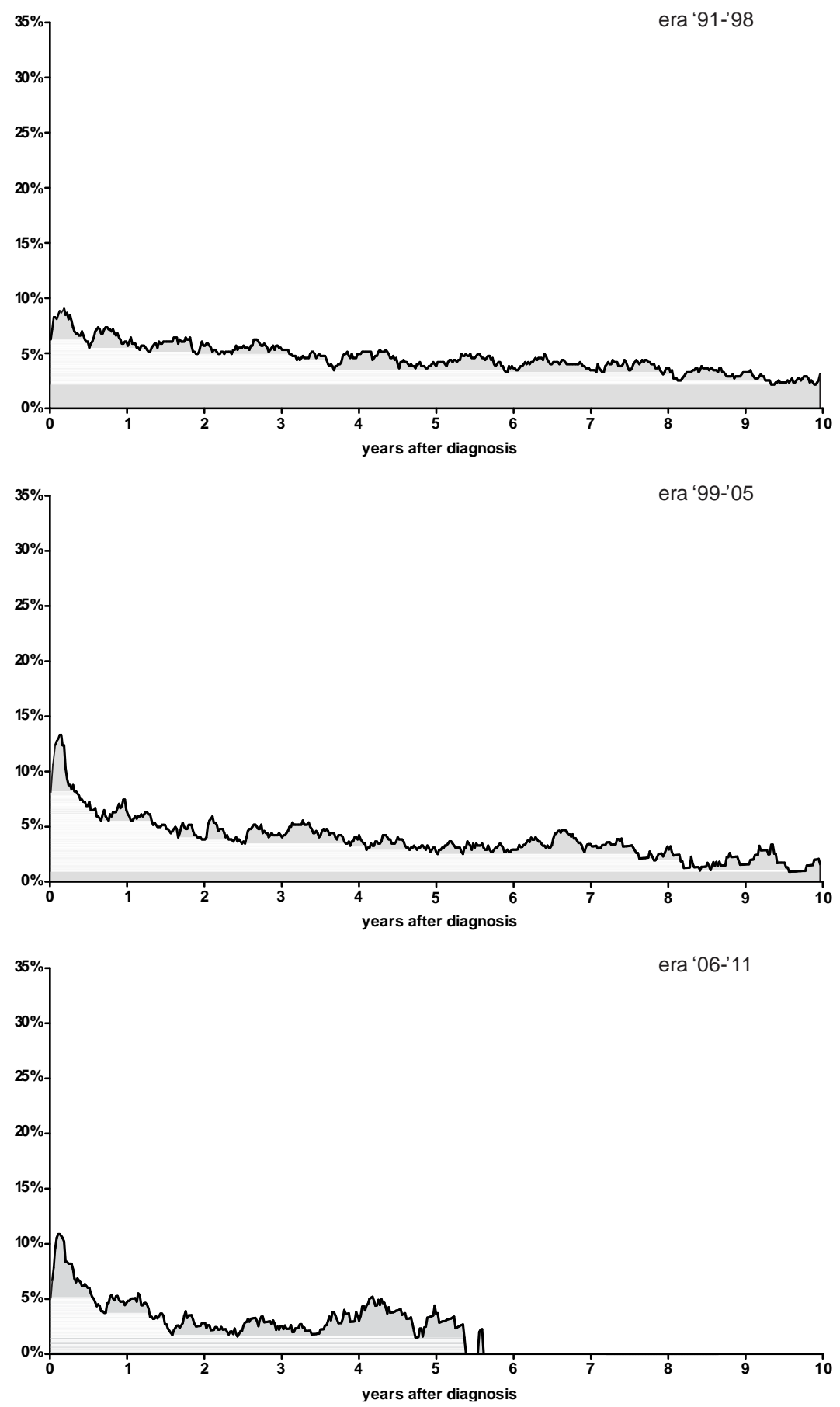

Figure 7.3. Point prevalence of systemic corticosteroid use in ulcerative colitis, stratified by era of diagnosis 


\section{Crohn's disease}

A Cumulative days of corticosteroids - year 1

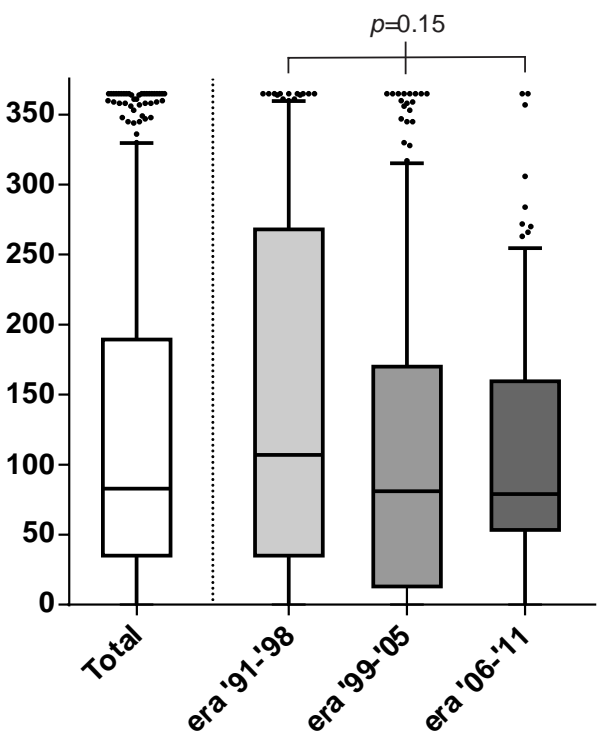

Ulcerative colitis

C Cumulative days of corticosteroids - year 1

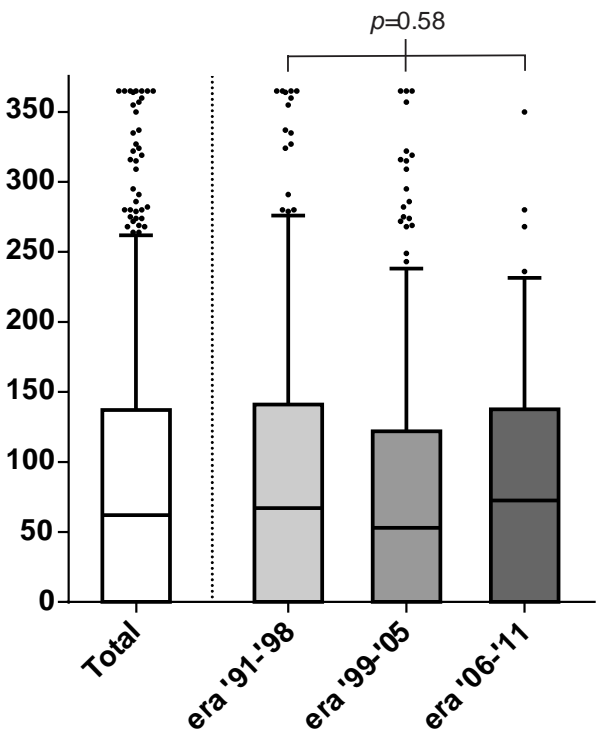

B Cumulative days of corticosteroids - year 5

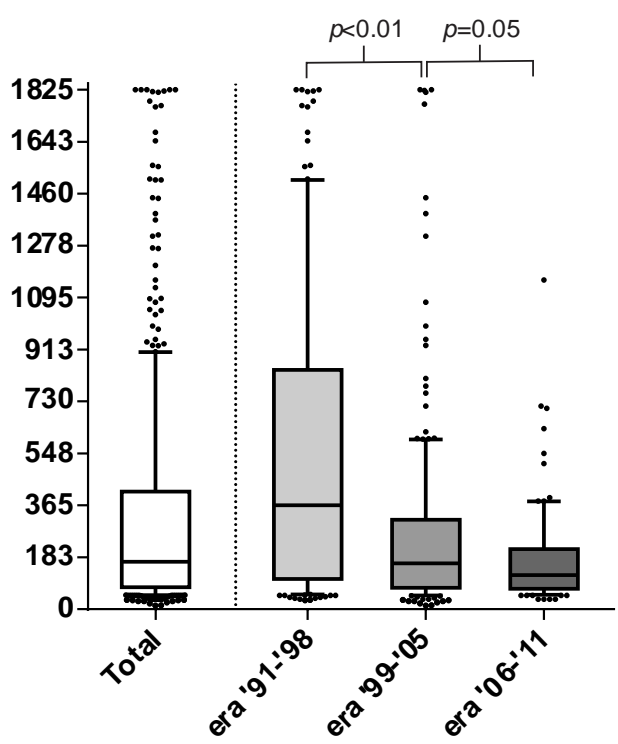

D Cumulative days of corticosteroids - year 5

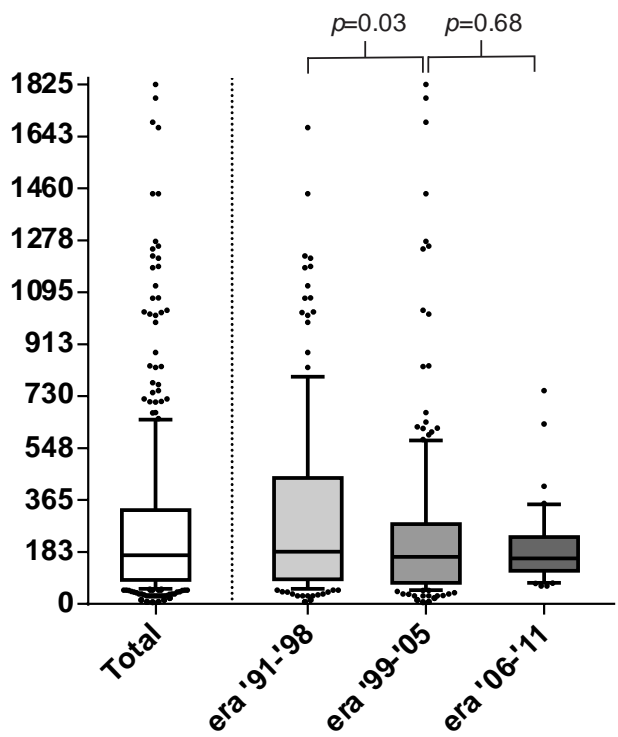

Figure 7.4. Cumulative corticosteroid use in the first year and in the first five years of disease in Crohn's disease [A and $\mathrm{B}$ ] and ulcerative colitis [C and D] 
Table 7.3A. Parameters associated with higher levels of cumulative corticosteroid use in Crohn's disease

\begin{tabular}{|c|c|c|c|}
\hline & $\begin{array}{l}\text { Cumulative 5-year } \\
\text { corticosteroid use }\end{array}$ & $\begin{array}{l}\text { Unadjusted } \\
\text { Odds ratio }\end{array}$ & $\begin{array}{l}\text { Adjusted } \\
\text { Odds ratio }\end{array}$ \\
\hline & median days [IQR] & OR $[95 \% \mathrm{Cl}]$ & \\
\hline \multicolumn{4}{|l|}{$\mathrm{Age}^{\mathrm{a}}$} \\
\hline $18-40$ years & 194 [91-504] & ref & ref \\
\hline 41-60 years & 119 [69-257] & $0.63[0.48-0.81]$ & $0.71[0.56-0.92]$ \\
\hline$>60$ years & 106 [69-205] & $0.62[0.42-0.93]$ & $0.64[0.43-0.96]$ \\
\hline \multicolumn{4}{|l|}{ Sex } \\
\hline male & 145 [82-399] & ref & ref \\
\hline female & $171[77-458]$ & $1.12[0.90-1.39]$ & $1.07[0.88-1.31]$ \\
\hline \multicolumn{4}{|l|}{ Era } \\
\hline 1991-1998 & $366[107-841]$ & ref & ref \\
\hline 1999-2005 & $161[75-314]$ & $0.54[0.43-0.67]$ & $0.54[0.44-0.68]$ \\
\hline 2006-2011 & $120[72-211]$ & $0.44[0.33-0.57]$ & $0.44[0.34-0.58]$ \\
\hline \multicolumn{4}{|l|}{ Disease locationa,b } \\
\hline L1, ileum & 129 [64-302] & ref & ref \\
\hline L2, colon & 191 [88-565] & $1.61[1.26-2.04]$ & $1.53[1.21-1.95]$ \\
\hline L3, ileocolon & $184[88-384]$ & $1.34[1.04-1.72]$ & $1.34[1.05-1.70]$ \\
\hline$\llcorner 4$, only upper $\mathrm{Gl}$ & $217[63-500]$ & $1.25[0.49-3.20]$ & $1.54[0.56-4.24]$ \\
\hline \multicolumn{4}{|l|}{ Disease behavior ${ }^{a, b}$} \\
\hline B1, inflammatory & 182 [84-497] & ref & ref \\
\hline $\mathrm{B} 2$, stricturing & $136[72-272]$ & $0.70[0.54-0.91]$ & $0.77[0.60-0.99]$ \\
\hline B3, penetrating & 168 [73-266] & $0.76[0.50-1.16]$ & 0.88 [0.58-1.33] \\
\hline \multicolumn{4}{|l|}{ Perianal disease ${ }^{a}$} \\
\hline No & 168 [82-497] & ref & ref \\
\hline Yes & $122[69-513]$ & $0.94[0.67-1.32]$ & $0.85[0.62-1.16]$ \\
\hline \multicolumn{4}{|l|}{ Upper GI locationa } \\
\hline No & $161[78-414]$ & ref & ref \\
\hline Yes & $209[101-378]$ & $1.05[0.74-1.50]$ & $1.07[0.75-1.53]$ \\
\hline \multicolumn{4}{|l|}{ Current smoker ${ }^{a}$} \\
\hline No & $131[76-314]$ & ref & ref \\
\hline Yes & $199[92-487]$ & $1.38[1.12-1.69]$ & $1.30[1.07-1.57]$ \\
\hline
\end{tabular}

95\% Cl, 95\% confidence interval; IQR, interquartile range; GI, gastrointestinal; ref, reference category

a at diagnosis

${ }^{\mathrm{b}}$ disease location according to Montreal consensus

analyses were performed in patients with a corticosteroid prescription within five years after diagnosis and a

follow-up of at least five years 


\begin{tabular}{|c|c|c|c|}
\hline & $\begin{array}{l}\text { Cumulative 5-year } \\
\text { corticosteroid use }\end{array}$ & $\begin{array}{l}\text { Unadjusted } \\
\text { Odds ratio }\end{array}$ & $\begin{array}{l}\text { Adjusted } \\
\text { Odds ratio }\end{array}$ \\
\hline & median days [IQR] & OR [95\%Cl] & OR [95\%Cl] \\
\hline \multicolumn{4}{|l|}{$\mathrm{Age}^{\mathrm{a}}$} \\
\hline $18-40$ years & 159 [84-342] & ref & ref \\
\hline 41-60 years & 169 [95-289] & $0.99[0.79-1.25]$ & $0.97[0.77-1.23]$ \\
\hline$>60$ years & 188 [73-358] & $0.98[0.76-1.26]$ & $0.96[0.75-1.24]$ \\
\hline \multicolumn{4}{|l|}{ Sex } \\
\hline male & 175 [91-353] & ref & ref \\
\hline female & $167[79-316]$ & $0.87[0.71-1.06]$ & $0.87[0.71-1.07]$ \\
\hline \multicolumn{4}{|l|}{ Era } \\
\hline 1991-1998 & 184 [86-443] & ref & ref \\
\hline 1999-2005 & $166[74-281]$ & $0.79[0.64-0.97]$ & 0.80 [0.65-0.99] \\
\hline 2006-2011 & $160[117-235]$ & $0.83[0.59-1.17]$ & $0.88[0.61-1.25]$ \\
\hline \multicolumn{4}{|l|}{ Disease locationa,b } \\
\hline E1, proctitis & 157 [76-272] & ref & ref \\
\hline E2, left-sided & 180 [98-384] & $1.29[0.99-1.69]$ & $1.24[0.95-1.63]$ \\
\hline E3, extensive & 158 [82-299] & $1.18[0.88-1.58]$ & $1.15[0.85-1.55]$ \\
\hline
\end{tabular}

$95 \% \mathrm{Cl}, 95 \%$ confidence interval; IQR, interquartile range; $\mathrm{Gl}$, gastrointestinal; ref, reference category

a at diagnosis

${ }^{b}$ disease location according to Montreal consensus

analyses were performed in patients with a corticosteroid prescription within five years after diagnosis and a follow-up of at least five years

The latter observation was found to be caused by a decrease in the cumulative corticosteroid use beyond the first year of disease: median 198 days [IQR 26-653] in era '91-'98 to median 63 days [IQR 0-173] in era '99-'05, to median 0 days [IQR 0-83] in era '06-'11, $p<0.01$.

In UC, corticosteroids users received a median of 62 days [IQR 0-137] of corticosteroids in the first year of disease, which was significantly lower compared to CD $[p<0.01]$. In the first five years, UC patients received a median of 171 days [IQR 84-330] of corticosteroids, which was not different from CD patients $[p=0.26]$. As in $C D$, the cumulative number of days on corticosteroids in the first year was found to be similar between eras [median 67 days [IQR 0-141] in era '91-'98, median 53 days [IQR 0-122] in era '99-'05, and median 73 days [IQR 0-138] in era '06-'11, $p=0.58]$, see Figure 7.4C. In contrast, the cumulative 5 -year use was significantly lower in era '99-'05 than in era '91 -'98 [ $\mathrm{p}=0.03]$, but did not further decrease in era '06-'11 [ $p=0.68]$, see Figure 7.4D and Table 7.3B. As in CD, the former decrease can be attributed to a decrease in cumulative corticosteroid use beyond the first year of disease [median 114 days [IQR 7-301] in the first and median 81 days [IQR $0-227]$ in the second era]. 
Other clinical parameters independently associated with a higher cumulative number of days of corticosteroid use in CD were: younger age at diagnosis, colonic involvement of the disease, inflammatory disease behavior, and being an active smoker at time of diagnosis, see Table 7.3A. In UC, none of the clinical parameters was significantly associated with higher levels of cumulative corticosteroids use, see Table 7.3B.

\section{Corticosteroid sparing effects of immunomodulators and anti-TNF $\alpha$ agents}

In the propensity score matched cohort on the corticosteroid sparing effects of immunomodulators, 313 CD users were matched to 313 CD nonusers and 114 UC users to 114 UC nonusers. Demographic and disease characteristics were well balanced between users and non-users [standardized differences $|<0.25|$ for all parameters] in both CD and UC and are presented in Supplement Tables $7 . S 1$ and 7.S2, respectively. In CD, the risk of corticosteroid exposure after the index date was $18.8 \%, 23.7 \%$, and $32.8 \%$ after 1,2 , and 5 years for immunomodulators users and $20.2 \%, 26.8 \%$, and $40.1 \%$ for nonusers, respectively [aHR 0.77; 95\% Cl 0.58-1.01]. The effect was stronger in CD patients commencing immunomodulator therapy within six months after diagnosis: aHR 0.63; 95\% Cl 0.47-0.85, with corresponding exposure rates of 22.0\%, 24.3\%, and 33.6\% [users] and $27.9 \%, 34.3 \%$, and $49.9 \%$ [nonusers] after 1,2, and 5 years after treatment start or index date, respectively. In UC, on the contrary, the corticosteroid exposure rate in users [17.2\%, 28.9\%, and 41.9\% after one, two, and five years] and nonusers [13.1\%, 18.2\%, and 37.6\%, respectively] was not different between groups [aHR 1.12; 95\% Cl 0.69-1.82].

In the propensity score matched cohort on the corticosteroid sparing effects of antiTNF $\alpha$ agents, 118 CD users were matched to 118 CD nonusers and 28 UC users to 28 UC nonusers. Groups were well balanced in demographic and disease characteristics [standardized differences $k 0.251$ for all parameters]. In CD, the risk of requiring corticosteroids after the index date was 13.2\% [one year], 14.2\% [two years], and 25.7\% [five years] in users as compared to $17.4 \%, 24.0 \%$, and $38.2 \%$, respectively, in nonusers [aHR 0.58; 95\% Cl 0.34-0.98]. For UC, numbers were limited, but no significant difference was found in the risk of requiring corticosteroids [HR 0.60; 95\% Cl 0.18-2.01]. 


\section{Discussion}

In this population-based study, we showed that the use of corticosteroids in IBD changed over the past two decades, in particular in CD. The number of IBD patients who ever required corticosteroid therapy remained stable, yet more CD patients already received a prescription in the first year of disease. The total amount of corticosteroids used was found to be stable in the first year of disease, while a divergence in days of use occurred after the first year, reflected by a lower cumulative corticosteroid use between the second and fifth year of disease and a lower corticosteroid point prevalence after the first disease year in more recent eras. Furthermore, we observed that immunomodulators and anti-TNF $\alpha$ agents are earlier and more frequently used and found that the use of these treatment modalities is associated with a lower risk of requiring corticosteroid therapy in real-life in CD.

This study illustrates that corticosteroid therapy remains a cornerstone in IBD management, even in the era of frequent and early use of immunomodulators and anti-TNFa therapy. We observed that $54.0 \%$ of the CD patients and $31.4 \%$ of the UC patients were exposed to corticosteroids within five years after diagnosis and found these rates to be stable over time. In literature, the corticosteroid exposure rate varies widely between studies, yet seems to be higher in hospital-based [57-75\% for $\mathrm{CD}^{5,35-38}, 45 \%-63 \%$ for $\mathrm{UC}^{35-38}$ ] than in population-based studies [43\%-76\% for $\mathrm{CD}^{6,23,25,39}, 34 \%-41 \%$ for UC $\left.6,23,40\right]$. Furthermore, exposure rates are higher in CD than in UC, which can be ascribed to the efficacy of 5 aminosalicylate therapy ${ }^{41}$ and the use of topical therapies in UC. ${ }^{42}$ We found an increase over time in corticosteroid exposure in the first year of disease in CD. It is likely that this is the consequence of good adherence to recent Dutch and European IBD guidelines, which advocate the use of corticosteroids for remission induction of the first flare of the disease in all patients, except for those with limited ileal disease localization. ${ }^{1,33}$

The total amount of corticosteroids given to a patient during disease course is an important clinical outcome measure, but has rarely been studied. In the present study, we found that more-recently diagnosed CD and UC patients have less cumulative days of corticosteroid use than patients diagnosed in the early days of our cohort and that this attenuation is caused by a less frequent use of corticosteroids after the first year of disease. Several factors are probably contributing to this effect. First, it is likely that an increasing awareness of the need for corticosteroid sparing and an improved insight in the long-term side effects of corticosteroid therapy played a role. The need for corticosteroid sparing is consequently underlined in recent IBD guidelines. ${ }^{1,2,33}$ Second, it is possible that sustained disease control has improved over time, resulting in a lower need for corticosteroid therapy. This may, for instance, be caused by better maintenance therapy and/or changes in the use of bowel surgery. Reflecting on the latter, in two previous studies on the temporal changes in 
the long-term outcome of IBD in the IBDSL cohort, we observed that the surgery rate in CD and the early colectomy rate in UC have decreased over time..$^{24,43}$ The paralleling trends of a declining surgery rate and a lower use of longstanding corticosteroid therapy, seem to indicate that refractory disease is better controlled nowadays. Another important factor is the increasing and earlier use of immunomodulators and anti-TNF $\alpha$ agents. In current guidelines, both immunomodulators and anti-TNF $\alpha$ agents are advocated as corticosteroid-sparing agents for $C D^{1,16,33,44}$ and $U C^{2,33,44,45}$. In two clinical $C D$ trials from the seventies, azathioprine was found to have a corticosteroid-sparing effect ${ }^{46-47}$, whereas no difference in corticosteroid requirement between azathioprine and placebo was found in the more recent AZTEC trial. ${ }^{48}$ In UC, the evidence for the efficacy of immunomodulators is less strong than in CD. Nevertheless, a meta-analysis of seven trials concluded that azathioprine is superior to placebo for maintaining corticosteroid-free remission ${ }^{18}$ and this conclusion was supported by a recent randomized study between azathioprine and 5 -aminosalicylate therapy. ${ }^{49}$ Data on the corticosteroid sparing effects of anti-TNF $\alpha$ agents is more consistent and illustrate that the risk of requiring corticosteroid therapy is lower in patients on anti-TNFa therapy than in patients on placebo. ${ }^{19-20}$ In the present study, we could confirm the corticosteroid sparing effect of immunomodulators and anti-TNFa agents for CD also in real-life, particularly if therapy is started early in disease course. Hence, it is likely that changes in medication played an important role in the decreasing use of corticosteroids after the first year of disease in CD. For UC, on the contrary, no corticosteroid-sparing effects of immunomodulator or anti-TNF $\alpha$ therapy were observed. However, it is important to acknowledge the limited number of patients in these analyses, possibly caused by the fact that UC patients are less often exposed to these treatment modalities and anti-TNF $\alpha$ therapy was registered at a later stage for UC. In the only other study evaluating the cumulative use of corticosteroids, Targownik et al. demonstrated that, in contrast to the present study, the cumulative corticosteroid use in IBD remained stable in the county of Manitoba [Canada] between 1995 and 2010, despite an increasing uptake of immunomodulator and anti-TNF $\alpha$ agents. ${ }^{34}$ Although it is likely that the use of corticosteroids in the management of $\mathrm{BD}$ is highly area dependent, a more frequent use of immunomodulator and biological therapy in our cohort over the Canadian cohort must be noted. In the Canadian cohort, the cumulative 5-year immunomodulator exposure increased from 19.8\% [1995-2000] to 31.7\% [2005-2009], whereas the exposure increased from 30.6\% [1991-1998] to 70.8\% [2006-2011] in our cohort. A similar difference was noted in the use of anti-TNF $\alpha$ agents: $5.1 \%$ to $12.7 \%$ [Canadian cohort] vs. $19.9 \%$ to $41.2 \%$ [IBDSL]. As a result, corticosteroid sparing effects can therefore be missed at population level. Future studies, in cohorts with a high uptake of immunomodulators and anti-TNF $\alpha$ therapy, preferably early in disease course, should confirm the results of the present study. 
Interestingly, in contrast to CD, the cumulative corticosteroid use showed no further decrease into the most-recent era in UC. This may be due to a more limited gain in corticosteroid sparing in UC, given the lower use of corticosteroids in UC than in CD. It may also be related to the observation that immunomodulator exposure in UC also stabilized between the second and third era [5-year exposure: $22.8 \%$ and $21.7 \%$, respectively]. Although no significant association was found between immunomodulator use and corticosteroid sparing in UC in this study, a clinical effect may have been missed due to the limited number of UC patients in the propensity score analyses.

Strengths of the present study reside in the large number of IBD patients included, its population-based character, and the long inclusion period. The latter two enabled us to determine the effects of the uptake of immunomodulators and anti-TNF $\alpha$ agents on corticosteroid use in an unselected IBD population. Despite its strengths, some limitations need to be addressed. First, data on corticosteroid use was retrieved directly from the medical records, rather than from pharmacies. This may have induced some inaccuracy, in particular in the duration of a course of corticosteroids, and has inevitably led to assumptions regarding missing or inconsistent data. Second, other factors than medication use may have contributed to the attenuated corticosteroid use during disease course, for example the advocacy of corticosteroid sparing in recent guidelines. The effects of such interventions are hard to study in an observational design and therefore must be kept in mind when interpreting the data. Third, residual confounding by indication may be present in the propensity score analyses. Such confounding would result in an underestimation of the true effect and may therefore partly explain the negative results concerning the corticosteroid sparing effects of immunomodulators in UC. Last, no firm conclusions can be drawn regarding the corticosteroid sparing effects of immunomodulators and anti-TNFa therapy in UC, given the low number of patients in the propensity score matched analyses. Future studies, with a longer follow-up time of UC patients in the era of anti-TNF $\alpha$ availability are therefore warranted.

In conclusion, corticosteroids are still frequently used in the first year of disease, but their cumulative use has decreased as of the second year of disease over the past two decades in a real-life IBD cohort. For CD, a significant association was found with the use of immunomodulators and anti-TNF $\alpha$ agents, which supports the increasing use of these treatment modalities in disease management. 


\section{Supplement}

\begin{tabular}{|c|c|c|c|c|c|c|c|}
\hline & & $\begin{array}{c}\text { IMM } \\
\text { nonuser } \\
{[\mathrm{N}=313]}\end{array}$ & $\begin{array}{c}\text { IMM } \\
\text { user } \\
{[\mathrm{N}=313]}\end{array}$ & & $\begin{array}{c}\text { anti-TNF } \alpha \\
\text { nonuser } \\
{[\mathrm{N}=118]}\end{array}$ & $\begin{array}{c}\text { anti-TNF } \alpha \\
\text { user } \\
{[N=118]}\end{array}$ & \\
\hline Age in years ${ }^{a}$ & mean [SD] & $35.2[14.7]$ & $35.5[14.9]$ & $p=0.83$ & $36.9[15.2]$ & $34.7[15.2]$ & $p=0.26$ \\
\hline Male & $N[\%]$ & $103[32.9]$ & $104[33$ & & 38 [32.2] & $35[29.7]$ & \\
\hline Current smoker & $N[\%]$ & $139[51.1]$ & $137[48.2]$ & $p=0.55$ & $44[40.0]$ & $50[48.1]$ & $p=0.27$ \\
\hline $\begin{array}{l}\text { Disease duration } \\
\text { in years }\end{array}$ & $\begin{array}{c}\text { median } \\
{[\mathrm{IQR}]}\end{array}$ & $\begin{array}{c}9.8 \\
{[5.4-15.1]}\end{array}$ & $\begin{array}{c}5.8 \\
{[3.9-9.0]}\end{array}$ & $p<0.01$ & $\begin{array}{c}7.1 \\
{[4.2-12.3]}\end{array}$ & $\begin{array}{c}4.8 \\
{[3.4-6.6]}\end{array}$ & $p<0.01$ \\
\hline Disease locationa,c & & & & $p=0.31$ & & & $p=0.85$ \\
\hline L1, ileum & $N[\%]$ & 138 [44.1] & $123(39.3)$ & & 39 [33.1] & 36 [30.5] & \\
\hline L2, colon & $N[\%]$ & $105[33.5]$ & $101(32$ & & 39 [33.1] & $43[36.4]$ & \\
\hline L3, ileocolon & $N[\%]$ & 69 [22.0] & $86(27.5)$ & & 40 [33.9] & 39 [33.1] & \\
\hline L4, only upper GI & $N[\%]$ & $1[0.3]$ & $3(1.0)$ & & - & - & \\
\hline Disease behavior ${ }^{c}$ & & & & $p=0.82$ & & & $p=0.92$ \\
\hline B1, inflammatory & $N[\%]$ & $220[70.3]$ & $226(72.2)$ & & 80 [67.8] & 82 [69.5] & \\
\hline B2, stricturing & $N[\%]$ & $56[17.9]$ & $50(16.0)$ & & 25 [21.2] & $22[18.6]$ & \\
\hline B3, penetrating & $N[\%]$ & $37[11.8]$ & $37(11.8)$ & & $13[11.0]$ & $14[11.9]$ & \\
\hline Perianal disease $e^{a}$ & $N[\%]$ & $29[9.3]$ & $23[7.3]$ & $p=0.47$ & 16 [13.6] & $15[12.7]$ & $p=0.99$ \\
\hline Upper Gl locationa & $N[\%]$ & 34 [10.9] & $42[13.4]$ & $p=0.39$ & 20 [16.9] & 20 [16.9] & $p=1.00$ \\
\hline
\end{tabular}

$\mathrm{SD}$, standard deviation; IQR, interquartile range; TNF $\alpha$, tumor necrosis factor $\alpha$

a at diagnosis

${ }^{\mathrm{b}}$ smoking status could not be retrieved in 169 cases

${ }^{c}$ disease location according to Montreal consensus

Table 7.S2. Patient characteristics of propensity-score matched ulcerative colitis patients

\begin{tabular}{|c|c|c|c|c|c|c|c|}
\hline & & $\begin{array}{c}\text { IMM } \\
\text { nonuser } \\
{[\mathrm{N}=114]}\end{array}$ & $\begin{array}{c}\text { IMM } \\
\text { user } \\
{[N=114]}\end{array}$ & & $\begin{array}{c}\text { anti-TNF } \alpha \\
\text { nonuser } \\
{[\mathrm{N}=28]}\end{array}$ & $\begin{array}{c}\text { anti-TNF } \alpha \\
\text { user } \\
{[\mathrm{N}=28]}\end{array}$ & \\
\hline Age in years ${ }^{a}$ & mean $[S D]$ & $43.2[17.3]$ & $42.6[16.2]$ & $p=0.80$ & $42.2[15.1]$ & $41.1[17.4]$ & $p=0.81$ \\
\hline Male & $\mathrm{N}[\%]$ & 63 [55.3] & 63 [55.3] & $p=1.00$ & 14 [50.0] & $14[50.0]$ & $p=1.00$ \\
\hline $\begin{array}{l}\text { Disease duration } \\
\text { in years }\end{array}$ & $\begin{array}{l}\text { median } \\
{[\mathrm{IQR}]}\end{array}$ & $\begin{array}{c}8.7 \\
{[5.6-15.4]}\end{array}$ & $\begin{array}{c}6.3 \\
{[3.4-10.4]}\end{array}$ & $p<0.01$ & $\begin{array}{c}9.2 \\
{[5.0-14.1]}\end{array}$ & $\begin{array}{c}3.1 \\
{[2.2-3.8]}\end{array}$ & $p<0.01$ \\
\hline Disease locationa,c & & & & $p=0.80$ & & & $p=0.77$ \\
\hline E1, proctitis & $N[\%]$ & 17 [15.0] & 13 [11.4] & & $6[21.4]$ & 5 [17.9] & \\
\hline E2, left-sided & $\mathrm{N}[\%]$ & $55[48.7]$ & $51[44.7]$ & & $16[57.1]$ & $14[50.0]$ & \\
\hline E3, extensive & $\mathrm{N}[\%]$ & 41 [36.3] & 50 [43.9] & & $6[21.4]$ & $9[32.1]$ & \\
\hline
\end{tabular}

$\mathrm{SD}$, standard deviation; IQR, interquartile range; TNF $\alpha$, tumor necrosis factor $\alpha$

a at diagnosis

${ }^{\mathrm{b}}$ disease location according to Montreal consensus

c disease location could not be retrieved in 13 cases

smoking data were not available for UC patients 


\section{References}

1. Dignass A, Van Assche G, Lindsay JO et al. The second European evidencebased Consensus on the diagnosis and management of Crohn's disease: current management. J Crohns Colitis 2010 ; 4: 28-62.

2. Dignass A, Lindsay JO , Sturm A et al. Second European evidence-based consensus on the diagnosis and management of ulcerative colitis part 2: current management. J Crohns Colitis $2012 ; 6: 991-1030$.

3. Truelove SC, Witts LJ . Cortisone in ulcerative colitis; final report on a therapeutic trial . Br Med J 1955;2: 1041-8

4. Summers RW, Switz DM, Sessions JT Jr. et al. National Cooperative Crohn's Disease Study: results of drug treatment Gastroenterology $1979 ; 77: 847-69$

5. Munkholm P, Langholz E, Davidsen M et al. Frequency of glucocorticoid resistance and dependency in Crohn's disease. Gut 1994; 35 : 360-2

6. Faubion WA Jr, Loftus EV Jr, Harmsen WS et al. The natural history of corticosteroid therapy for inflammatory bowel disease: a population-based study Gastroenterology $2001 ; 121: 255-60$

7. Ford AC, Bernstein CN, Khan KJ et al. Glucocorticosteroid therapy in inflammatory bowel disease: systematic review and meta-analysis. Am J Gastroentero/2011;106:590-9

8. Lennard-Jones JE, Misiewicz JJ , Connell AM et al. Prednisone as maintenance treatment for ulcerative colitis in remission. Lancet 1965;1:1889

9. Steinhart AH, Ewe K, Griffiths AM et al. Corticosteroids for maintenance of remission in Crohn's disease. Cochrane Database Syst Rev 2003 , Cd000301

10. Buchman AL. Side effects of corticosteroid therapy. J Clin Gastroentero/2001;33:289-94 .
11. Curtis JR, Westfall AO, Allison J et al. Population-based assessment of adverse events associated with long-term glucocorticoid use. Arthritis Rheum $2006 ; 55: 420-6$

12. Hoes JN, Jacobs JW, Verstappen SM et al. Adverse events of low- to mediumdose oral glucocorticoids in inflammatory diseases: a meta-analysis . Ann Rheum Dis 2009 ; 68:1833-8.

13. Lichtenstein GR, Feagan BG , Cohen RD et al. Serious infection and mortality in patients with Crohn's disease: more than 5 years of follow-up in the TREAT registry . Am J Gastroentero/ 2012;107; 1409-22

14. Katz JA. Treatment of inflammatory bowel disease with corticosteroids Gastroenterol Clin North Am 2004 ;33: 171-89

15. Irving PM, Gearry RB, Sparrow MP et al. Review article: appropriate use of corticosteroids in Crohn's disease . Aliment Pharmacol Ther 2007; 26:31329.

16. Lichtenstein GR, Hanauer SB, Sandborn WJ et al. Management of Crohn's disease in adults. Am J Gastroenterol 2009; 104: 465-83

17. Chande N, Patton PH, Tsoulis DJ et al. Azathioprine or 6-mercaptopurine for maintenance of remission in Crohn's disease . Cochrane Database Syst Rev 2015, CD000067

18. Timmer A, Patton PH , Chande $\mathrm{N}$ et al. Azathioprine and 6-mercaptopurine for maintenance of remission in ulcerative colitis . Cochrane Database Syst Rev 2016, CD000478.

19. Akobeng AK, Zachos M. Tumor necrosis factor-alpha antibody for induction of remission in Crohn's disease. Cochrane Database Syst Rev 2004 ,CD003574 .

20. Behm BW, Bickston SJ. Tumor necrosis factor-alpha antibody for maintenance of remission in Crohn's disease . 
Cochrane Database Syst Rev 2008 , CD006893.

21. Lawson MM , Thomas AG, Akobeng AK . Tumour necrosis factor alpha blocking agents for induction of remission in ulcerative colitis. Cochrane Database Syst Rev 2006 , CD005112.

22. Salleron $J$, Danese $S, D^{\prime}$ Agay $L$ et al. Effectiveness research in inflammatory bowel disease: a necessity and a methodological challenge. J Crohns Colitis 2016; 10: 1096-102.

23. Rungoe $C$, Langholz $E$, Andersson $M$ et al. Changes in medical treatment and surgery rates in inflammatory bowel disease: a nationwide cohort study 1979-2011. Gut 2014; 63: 1607-16.

24. Jeuring SF, Bours PH, Zeegers MP et al. Disease outcome of ulcerative colitis in an era of changing treatment strategies: results from the Dutch population-based IBDSL cohort. J Crohns Colitis 2015; 9 837-45.

25. Ramadas AV, Gunesh S, Thomas GA et al. Natural history of Crohn's disease in a population-based cohort from Cardiff (1986-2003): a study of changes in medical treatment and surgical resection rates. Gut 2010; 59: 1200-6.

26. Lakatos PL, Golovics PA, David G et al. Has there been a change in the natural history of Crohn's disease? Surgical rates and medical management in a population-based inception cohort from Western Hungary between 1977-2009 . Am J Gastroentero/ 2012; 107: 57988.

27. Zhulina $Y$, Udumyan $R$, Tysk $C$ et al. The changing face of Crohn's disease: a population-based study of the natural history of Crohn's disease in Orebro, Sweden 1963-2005. Scand J Gastroenterol2016; 51: 304-13.

28. Magro F, Rodrigues-Pinto E, Coelho R et al. Is it possible to change phenotype progression in Crohn's disease in the era of immunomodulators? Predictive factors of phenotype progression. Am J Gastroentero/2014; 109: 1026-36.
29. Safroneeva E, Vavricka SR, Fournier N et al. Impact of the early use of immunomodulators or TNF antagonists on bowel damage and surgery in Crohn's disease. Aliment Pharmacol Ther 2015; 42: 977-89.

30. van den Heuvel TR, Jonkers DM Jeuring SF et al. Cohort profile: the Inflammatory Bowel Disease South Limburg Cohort [IBDSL] . Int J Epidemiol 2015; 46: e7.

31. Silverberg MS, Satsangi J , Ahmad T et al. Toward an integrated clinical, molecular and serological classification of inflammatory bowel disease: report of a Working Party of the 2005 Montreal World Congress of Gastroenterology. Can J Gastroentero/ 2005; 19 [Suppl A]: 5A-36A.

32. Satsangi $J$, Silverberg MS, Vermeire $S$ et al. The Montreal classification of inflammatory bowel disease: controversies, consensus, and implications. Gut 2006; 55: 749-53.

33. van Bodegraven AA, van Everdingen $\mathrm{JJ}$, Dijkstra G et al. [Guideline 'Diagnosis and treatment of inflammatory bowel disease in adults'. I. Diagnosis and treatment]. Ned Tijdschr Geneeskd 2010; 154: A1899.

34. Targownik LE, Nugent $Z$, Singh $H$ et al. Prevalence of and outcomes associated with corticosteroid prescription in inflammatory bowel disease. Inflamm Bowel Dis 2014; 20: 622-30.

35. Ho GT, Chiam P, Drummond $\mathrm{H}$ et al. The efficacy of corticosteroid therapy in inflammatory bowel disease: analysis of a 5-year UK inception cohort. Aliment Pharmacol Ther 2006; 24: 319-30.

36. Chow DK, Sung JJ, Tsoi KK et al. Predictors of corticosteroid-dependent and corticosteroid-refractory inflammatory bowel disease: analysis of a Chinese cohort study. Aliment Pharmacol Ther 2009; 29: 843-54.

37. Shapiro JM , Hagin SE, Shah SA et al. corticosteroid use in a prospective, community-based cohort of newly diagnosed inflammatory bowel disease 
patients. Dig Dis Sci2016; 61 : 1635 40 .

38. Khan N, Abbas A, Williamson A et al. Prevalence of corticosteroids use and disease course after initial steroid exposure in ulcerative colitis. Dig Dis Sci 2013; 58: 2963-9.

39. Solberg IC, Vatn MH, Hoie O et al. Clinical course in Crohn's disease: results of a Norwegian population-based tenyear follow-up study. Clin Gastroenterol Hepato/2007; 5: 1430-8.

40. Solberg IC, Lygren I, Jahnsen J et al. Clinical course during the first 10 years of ulcerative colitis: results from a population-based inception cohort [IBSEN Study] . Scand J Gastroenterol $2009 ; 44: 431-40$.

41. Sutherland LR, May GR, Shaffer EA . Sulfasalazine revisited: a meta-analysis of 5-aminosalicylic acid in the treatment of ulcerative colitis. Ann Intern Med $1993 ; 118: 540-9$.

42. Scholmerich J. Review article: systemic and topical steroids in inflammatory bowel disease. Aliment Pharmacol Ther 2004; 20 [Suppl 4]:66 -74.

43. Jeuring SF, van den Heuvel TR, Liu LY et al. Improvements in the long term outcome of Crohn's disease over the past two decades and the relation to changes in medical management: results from the population-based IBDSL cohort. Am J Gastroentero/ 2017 ; 112:32536.

44. Mowat C, Cole A, Windsor A et al. Guidelines for the management of inflammatory bowel disease in adults . Gut 2011; 60: 571-607.

45. Kornbluth A, Sachar DB. Practice Parameters Committee of the American College of $G$. Ulcerative colitis practice guidelines in adults: American College Of Gastroenterology, Practice Parameters Committee. Am J Gastroentero/ 2010; 105: 501-23

46. Rosenberg JL, Levin B, Wall AJ et al. A controlled trial of azathioprine in Crohn's disease. Am J Dig Dis 1975; 20: 721-6
47. Willoughby JM, Beckett J , Kumar PJ et al. Controlled trial of azathioprine in Crohn's disease. Lancet 1971; 2: 944-7

48. Panes J , Lopez-Sanroman A, Bermejo F et al. Early azathioprine therapy is no more effective than placebo for newly diagnosed Crohn's disease . Gastroenterology 2013; 145: 766-74

49. Ardizzone S, Maconi G, Russo A et al. Randomised controlled trial of azathioprine and 5-aminosalicylic acid for treatment of steroid dependent ulcerative colitis. Gut 2006 ; 55: 47-53. 



\section{CHAPTER 8}

\section{Time trends in the epidemiology and outcome of perianal fistulizing Crohn's disease in a population-based cohort}

Kevin WA Göttgens*, Steven FG Jeuring*, Rosel Sturkenboom, Marielle JL Romberg-Camps, Liekele E Oostenbrug, Daisy MAE Jonkers, Laurents PS Stassen, Ad AM Masclee, Marieke J Pierik, Stéphanie O Breukink

* both authors contributed equally to this work

Eur J Gastroenterol Hepatol. 2017 May;29(5):595-601 


\section{Abstract}

\section{Background}

Perianal disease is a debilitating condition that frequently occurs in Crohn's disease [CD] patients. It is currently unknown whether its incidence has changed in the era of frequent immunomodulator use and biological availability. We studied the incidence and outcome of perianal and rectovaginal fistulas over the past two decades in our population-based Inflammatory Bowel Disease South Limburg cohort.

\section{Methods}

All 1162 CD patients registered in the Inflammatory Bowel Disease South-Limburg registry were included. The cumulative probabilities of developing a perianal and rectovaginal fistula were compared between three eras distinguished by the year of CD diagnosis: 1991-1998, 1999-2005 and 2006-2011. Second, clinical risk factors and the risk of fistula recurrence were determined.

\section{Results}

The cumulative 5 -year perianal fistula rate was $14.1 \%$ in the $1991-1998$ era, $10.4 \%$ in the 1999-2005 era and $10.3 \%$ in the 2006-2011 era, $p=0.70$. Colonic disease was associated with an increased risk of developing perianal disease, whereas older age was associated with a decreased risk [both $p<0.01$ ]. Over time, more patients were exposed to immunomodulators or biologicals before fistula diagnosis [18.5 vs. 32.1 vs. $52.1 \%$, respectively, $p=0.02$ ] and started biological therapy thereafter [ 18.6 vs. 34.1 vs. $54.0 \%$, respectively, $p<0.01]$. The cumulative 5 -year perianal fistula recurrence rate was not significantly different between eras [19.5 vs. 25.5 vs. $33.1 \%, p=0.28$ ]. In contrast, the cumulative 5 -year rectovaginal rate attenuated from $5.7 \%$ [the 1991-2005 era] to $1.7 \%$ [the 2006-2011 era], $p=0.01$.

\section{Conclusion}

Over the past two decades, the risk of developing a perianal fistula was stable, as well as its recurrence rate, underlining the lasting need for improving treatment strategies for this invalidating condition. 


\section{Introduction}

Crohn's disease [CD] is a chronic inflammatory disease that can affect the entire gastrointestinal tract, including the anus. Perianal CD is present in 10-37\% of patients at the time of diagnosis and may even be the presenting symptom. ${ }^{1-3}$ Perianal CD is an umbrella term for perianal abscesses, skin tags, fissures, strictures and fistulas ${ }^{4}$. Perianal fistulizing CD comprise perianal fistulas [PF] and rectovaginal fistulas [RVF] and is probably the most debilitating form of perianal CD. The natural history is characterized by frequent episodes of ductal discharge, abscess formation and perianal pain, often impairing patients' daily functioning and quality of life. ${ }^{5}$

Several cohort studies have investigated the incidence of perianal CD. Populationbased studies are preferred when studying the epidemiology of fistulizing CD because selection bias is likely to occur in referral center studies as perianal fistulas are often treatment refractory and, as a result, patients are often referred to specialized centers. In populationbased studies, the incidence of PFs in CD patients is estimated to be between 13 and 28\%. ${ }^{1,6-9}$ Most incidence and outcome data derive from studies that recruited patients decades ago. An update of these data has recently been considered an 'unmet need' in the field of $\mathrm{PFs}^{10}$ and is needed to assess the risk of developing fistulizing disease in an era in which immunomodulators and biologicals are more frequently used in the medical management of CD.

Therefore, the aim of this study was to assess the incidence of PFs in our populationbased inflammatory bowel disease [IBD] cohort and to study the evolution thereof in patients diagnosed between 1991 and 2011, followed until 2014. Second, we aimed to study the incidence of RVFs specifically, to identify clinical risk factors for PF, and to assess changes in PF outcome over the past two decades. 


\section{Methods}

\section{Cohort description and study population}

The IBD South Limburg [IBDSL] cohort is a population-based IBD cohort in the well-defined South Limburg area of the Netherlands, with a completeness of over 93\%. The cohort has been described in detail previously. ${ }^{11-12}$ In brief, incident adult IBD cases in the area are being registered as from 1991 and data on demographics, disease phenotype, IBD medication, IBDrelated complications [including fistulas], hospitalizations and surgeries are being collected. All data are retrieved directly from the medical files using standardized registration forms. For this study, all 1162 CD patients in the IBDSL registry were included. These patients were diagnosed between January 1991 and July 2011 at the age of 18 years or older. Patients were followed from the date of diagnosis to the end of data collection [2014], migration out of the area or death. IBDSL has been approved by the Ethics Committee of the Maastricht University Medical Center [NL31636.068.10] and is registered in https://clinicaltrials.gov [NCT02130349].

\section{Study endpoints}

The primary endpoint of the study was the evolution of the incidence of PF in CD patients over the past two decades. To assess the time trend, the full cohort [1991-2011] was equally divided into three separate eras, also considering the registration of anti-tumor necrosis factor-alpha [anti-TNFa] agents in the Netherlands in 1999. The 1991-1998 era included patients with a CD diagnosis between 1991 and 1998, the 1999-2005 era included patients diagnosed between 1999 and 2005 and the 2006-2011 era included patients diagnosed between 2006 and 2011. The secondary endpoints were time trends in the incidence of RVFs, clinical risk factors for PFs and RVFs, and the evolution of the outcome of PFs in terms of the need for surgery and fistula recurrence. Furthermore, type of IBD therapy before fistula diagnosis was noted, even as changes in CD therapy after fistula diagnosis. Treatment status was determined considering all fistulas that occurred during the course of $\mathrm{CD}$ and considering fistulas that developed within 5 years after $C D$ diagnosis to adjust for differences in the maximum follow-up of the three eras.

\section{Definitions}

PFs and RVFs were identified by a thorough review of the medical records of all CD patients. Fistulas diagnosed by either radiologic imaging or a physical examination were included for study. If imaging data [or endoanal ultrasound] were available, the PF was subsequently classified by two authors [K.G./S.B.], including an experienced gastrointestinal surgeon, or the classification was extracted from the radiologic report. In case classification was not possible, 
because radiologic imaging was not performed or unavailable, the classification was coded 'unknown'. PFs were classified according to [l] its relation to the sphincter muscle [low or high fistula $]^{13}$, [II] the Park classification ${ }^{14}$ and [III] the simple/complex classification. ${ }^{4}$ A low fistula was defined as a fistula involving the lower one-third of the sphincter complex and a high fistula was defined as a fistula traversing the middle and/or the upper third part. ${ }^{13} \mathrm{~A}$ fistula was classified as a simple fistula if a low superficial, intersphincteric or transsphincteric fistula was observed without complicating factors such as multiple tracts, abscesses or strictures. A complex fistula is a simple fistula with the aforementioned complications or a high fistula. ${ }^{4}$ RVFs were described separately in this study and classified as either a low or a high fistula. Low fistulas involve the lower half of the rectovaginal septum and high fistulas involved the upper half. In this study, a fistula recurrence was defined as either a visible new fistula at the same location or the return of symptoms after a symptom-free period. Disease phenotype was classified according to the Montreal consensus. ${ }^{15-16}$ In contrast to the older Vienna classification ${ }^{17}$, PFs are not included in the definition of a penetrating disease behavior [B3], but classified separately.

\section{Statistical analyses}

Continuous data are presented as means with SDs or medians with interquartile ranges [IQR], depending on the normality of the underlying distribution, and subsequently compared between groups using an independent Student's t-test or the Mann-Whitney U-test, respectively. Discrete data are presented as absolute numbers with percentages and subsequently compared between groups using $x^{2}$-tests. Kaplan-Meier survival analyses were used to estimate the cumulative probabilities of developing a PF or an RVF over time to adjust for follow-up differences between patients. PFs and RVFs present before CD diagnosis were described separately, but considered to have developed at the time of diagnosis in the cumulative probability analyses to include these fistulas in the cumulative incidence rates. Cox regression models were established to assess associations between fistula incidence and era of diagnosis, adjusting for age at diagnosis, sex, disease location and disease behavior. Because of the low number of RVFs in the cohort, only univariable models could be used for RVF analyses. Cox regression model data were expressed as hazard ratios [HR] with accompanying 95\% confidence intervals [95\% Cl]. A p-value lower than 0.05 was considered statistically significant. All statistical analyses were carried out using SPSS, version 21 [IBM Corp., Armonk, New York, USA]. 


\section{Results}

In the IBDSL database, 1162 CD patients were identified. The patient group included 728 [62.7\%] female patients. The mean age at CD diagnosis was 37.7 [SD 15.9] years and the mean follow-up duration was 8.7 [SD 5.7] years. Of the 1162 patients, 316 patients were diagnosed in the 1991-1998 era, 387 patients in the 1999-2005 era and 459 patients in the 2006-2011 era. Over time, the cumulative 1-year and 5-year exposure to immunomodulators increased from 9.1 and 30.6\% [the 1991-1998 era] to 31.0 and $56.7 \%$ [the 1999-2005 era] to 49.5 and $70.8 \%$ [the 2006-2011 era], $p<0.01$. Similarly, the cumulative 1-year and 5-year exposure to anti-TNF $\alpha$ agents increased: 0 and $3.1 \%$ in the 1991-1998 era, 7.1 and $19.9 \%$ in the $1999-2005$ era and 21.2 and $41.2 \%$ in the $2006-$ 2011 era, $p<0.01$. We identified 284 [24.4\%] patients with fistulizing disease [including perianal, rectovaginal, enterocutaneous and enteroenteral fistulas]. The number of patients with a PF and/or an RVF was 163 [14.0\%]. Of these patients, 161 [13.9\%] ever had a PF, 17 [2.3\%] women ever had an RVF and 15 [2.1\%] women ever suffered from both a PF and an RVF. Patient characteristics of CD patients with and without fistulas are shown in Table 8.1 .

\begin{tabular}{|c|c|c|c|c|c|}
\hline & & $\begin{array}{c}\text { CD patients } \\
\text { without PF/RVF } \\
{[N=995]}\end{array}$ & $\begin{array}{c}\text { CD patients } \\
\text { with only a PF } \\
{[N=150]}\end{array}$ & $\begin{array}{c}\text { CD patients } \\
\text { with a RVF } \\
{[N=17]}\end{array}$ & \\
\hline Age in years ${ }^{a}$ & mean [SD] & $38.5[16.3]$ & $32.3[12.5]$ & $37.3[15.1]$ & $p_{1}<0.01, p_{2}=0.78$ \\
\hline Female & $N[\%]$ & $620[62.3]$ & $91[60.7]$ & $17[100]$ & $p_{1}=0.72$ \\
\hline Current smoker ${ }^{a, b}$ & $\mathrm{~N}[\%]$ & $420[49.8]$ & 70 [49.6] & $7[46.7]$ & $p_{1}=0.99$ \\
\hline Follow-up in years & mean $[S D]$ & $8.3[5.6]$ & $11.1[5.8]$ & $13.6[5.8]$ & $p_{1}<0.01, p_{2}<0.01$ \\
\hline Disease location ${ }^{a}$ & & & & & $p_{1}=0.03, p_{2}=0.23$ \\
\hline$L 1$, ileum & $N[\%]$ & $448[45.0]$ & 48 [32.0] & $4[23.5]$ & \\
\hline L2, colon & $N[\%]$ & $305[30.7]$ & $58[38.7]$ & $8[47.1]$ & \\
\hline L3, ileocolon & $N[\%]$ & $222[22.3]$ & $40[26.7]$ & $5[29.4]$ & \\
\hline$\llcorner 4$, only upper $G \mid$ & $N[\%]$ & $20[2.0]$ & $4[2.7]$ & - & \\
\hline Disease behavior ${ }^{\mathrm{a}}$ & & & & & $p_{1}=0.52, p_{2}=0.20$ \\
\hline B1, inflammatory & $N$ [\%] & $773[77.7]$ & 116 [77.3] & 11 [64.7] & \\
\hline B2, stricturing & $\mathrm{N}[\%]$ & $148[14.9]$ & $26[17.3]$ & $3[17.6]$ & \\
\hline B3, penetrating & $N[\%]$ & $74[7.4]$ & $8[5.3]$ & $3[17.6]$ & \\
\hline
\end{tabular}

$\mathrm{CD}$, Crohn's disease; PF, perianal fistula; RVF, rectovaginal fistula; $\mathrm{SD}$, standard deviation $p_{1}$ - $p$-value of the comparison CD patients without PF/RVF versus CD patients with a PF $p_{2}$ - $p$-value of the comparison CD patients without PF/RVF versus CD patients with a RVF ${ }^{a}$ at diagnosis

${ }^{\mathrm{b}}$ smoking status was not available in 169 cases 


\section{Perianal fistula incidence}

In total, 161 [13.9\%] CD patients ever had a PF. The classifications of these fistulas are shown in Table 8.2.

\begin{tabular}{|c|c|c|c|c|}
\hline & $\begin{array}{c}\text { All primary } \\
\text { perianal fistulas } \\
{[\mathrm{N}=161]}\end{array}$ & $\begin{array}{c}\text { Operated primary } \\
\text { perianal fistulas } \\
\qquad[N=95]\end{array}$ & $\begin{array}{c}\text { Perianal fistula } \\
\text { recurrences } \\
{[\mathrm{N}=59]}\end{array}$ & $\begin{array}{c}\text { Rectovaginal } \\
\text { fistulas } \\
{[N=17]}\end{array}$ \\
\hline \multicolumn{5}{|l|}{ Low/high classification } \\
\hline low fistula, $N[\%]$ & 54 [33.5] & 34 [35.8] & $11[18.6]$ & $6[35.3]$ \\
\hline high fistula, $N[\%]$ & $36[22.4]$ & $32[33.7]$ & $14[23.7]$ & $2[11.8]$ \\
\hline unknown, N $[\%]$ & $71[44.1]$ & 29 [30.5] & $34[57.6]$ & 9 [52.9] \\
\hline \multicolumn{5}{|l|}{ Park's classification } \\
\hline intersphincteric, $N[\%]$ & 36 [22.4] & 22 [23.2] & $14[23.7]$ & - \\
\hline transsphincteric, $N[\%]$ & 30 [18.6] & $20[21.1]$ & $4[6.8]$ & - \\
\hline extrasphincteric, $N[\%]$ & $12[7.5]$ & $11[11.6]$ & $3[5.1]$ & - \\
\hline suprasphincteric, $N[\%]$ & $7[4.3]$ & $6[6.3]$ & $3[5.1]$ & - \\
\hline superficial, $N[\%]$ & $15[9.3]$ & $7[7.4]$ & $2[3.4]$ & - \\
\hline unknown, $N[\%]$ & $61[37.9]$ & 29 [30.5] & $33[55.9]$ & - \\
\hline \multicolumn{5}{|l|}{ Simple/complex classification } \\
\hline simple, $N[\%]$ & 39 [24.2] & $20[21.1]$ & $12[20.3]$ & - \\
\hline complex ${ }^{\mathrm{a}}, N[\%]$ & 39 [24.2] & 29 [30.5] & 9 [15.3] & - \\
\hline unknown, $N[\%]$ & $83[51.6]$ & $46[48.4]$ & 38 [64.4] & - \\
\hline
\end{tabular}

a rectovaginal fistulas were not considered complex perianal fistulas, but described separately

Forty-nine [30.4\%] patients developed the fistula before CD diagnosis at a median time of 0.8 [IQR 0.2-2.7] years before diagnosis. The majority [ $n=112,69.6 \%]$ developed a PF during the course of $C D$ at a median time of 2.1 [IQR 0.4-6.3] years after diagnosis. The overall cumulative probability of developing a PF during the course of the disease was $8.3 \%$ after 1 year, $11.6 \%$ after 5 years and $15.8 \%$ after 10 years. The cumulative 5 -year probability of developing a PF was not different between the three eras [14.1\% in the 1991-1998 era, $10.4 \%$ in the 1999-2005 era and $10.3 \%$ in the 2006-2011 era, $p=0.70$; Figure 8.1A]. 
A

Cumulative probability of developing perianal fistula

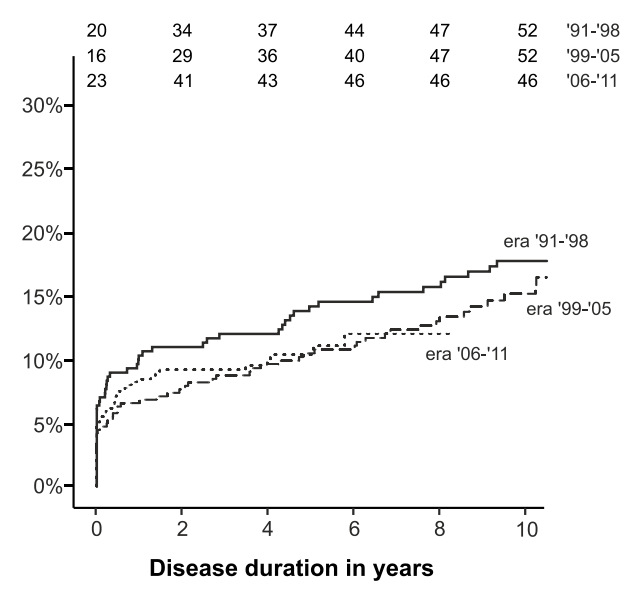

$\begin{array}{llllcccc}\mathrm{N} \text { at risk } & 316 & 262 & 248 & 229 & 211 & 193 & \text { '91-'98 } \\ \mathrm{N} \text { at risk } & 387 & 340 & 316 & 297 & 251 & 144 & \text { '99-'05 } \\ \mathrm{N} \text { at risk } & 459 & 356 & 216 & 77 & 4 & 0 & \text { '06-'11 }\end{array}$
B

Cumulative probability of developing fistula recurrence

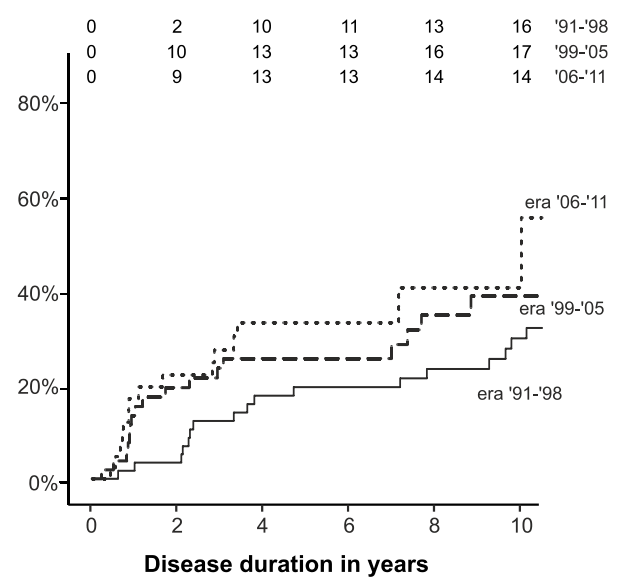

Figure 8.1. Kaplan-Meier survival curves showing the cumulative probability of developing a primary perianal fistula $[\mathrm{A}]$ and a perianal fistula recurrence $[\mathrm{B}]$.

Results were concordant in the multivariable model, adjusted for age, sex, disease location and disease behavior $[p=0.72]$. A colonic or an ileocolonic disease location at diagnosis was associated with an increased risk of developing a PF [adjusted HR: 1.90; 95\% Cl: 1.29-2.81 and adjusted HR: 1.60; 95\% Cl: 1.06-2.41, respectively]. In addition, the cumulative 5-year PF risk was found to be the lowest in patients who were older in age at CD diagnosis: 18-40 years, 13.6\%, 40-60 years, 8.5\% and older than 60 years, 6.2\% [ $p<0.01$; Table 8.3]. Medication status at PF diagnosis is shown in Figure 8.2, stratified by era. Considering PFs that were diagnosed within 5 years after diagnosis $[n=78]$, an increasing number of patients had been exposed to immunomodulator and anti-TNFa agents before the diagnosis of PF: $18.5 \%$ [the 1991-1998 era] versus 32.1\% [the 1999-2005 era] versus $52.1 \%$ [the $2006-$ $2011 \mathrm{era}$ ], $p=0.02$. In particular, the number of patients with previous exposure to anti-TNF $\alpha$ agents increased: 0\% [the 1991-1998 era] versus 0\% [the 1999-2005 era] versus 26.0\% [the 2006-2011 era] [ $p<0.01]$. 


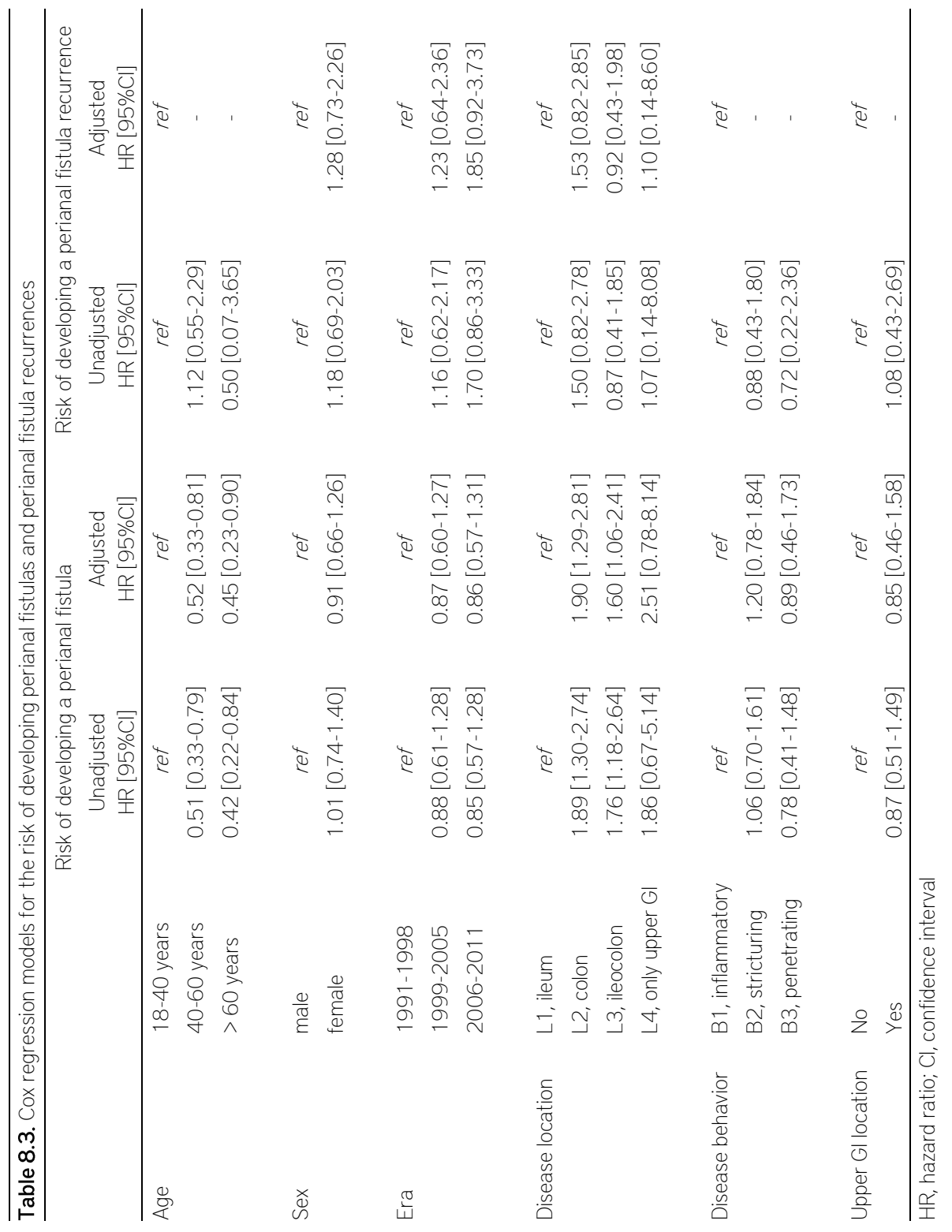


A Medication use before perianal fistula diagnosis

Era 1991-1998

$(n=43)$

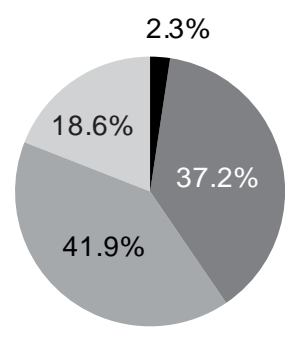

Era 1999-2005

$(n=44)$

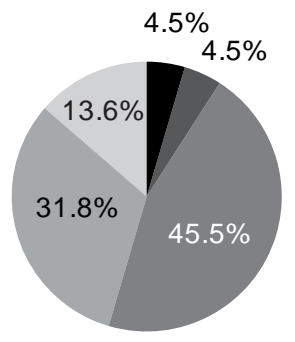

Era 2006-2011

$(n=25)$

\section{B Medication use after perianal fistula diagnosis}

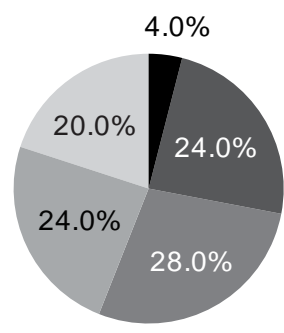

Therapy naive or no medication use

Current or past 5-ASA monotherapy

Current or past IMM monotherapy

Current or past anti-TNFa monotherapy

Current or past combination therapy

$$
\underset{(n=43)}{\text { Era }}
$$

Era 1999-2005

$(n=44)$
Era 2006-2011

$(n=25)$
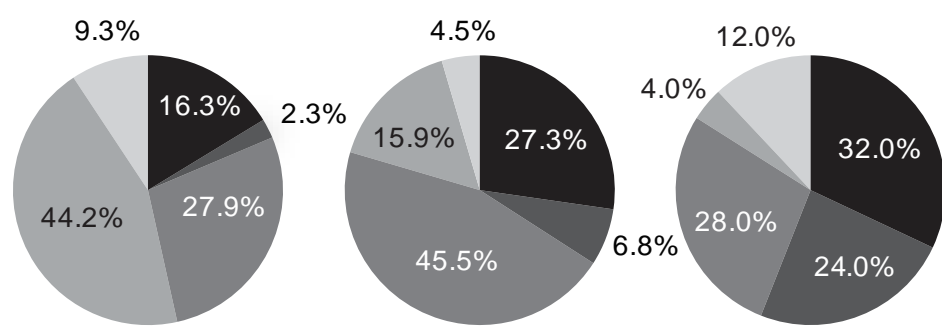

Therapy naive or no medication use

Current or past 5-ASA monotherapy

Current or past IMM monotherapy

Current or past anti-TNFa monotherapy

Current or past combination therapy

Figure 8.2. Medication use before $[A]$ and after $[B]$ the diagnosis of perianal fistulas that developed during the course of the disease.

5-ASA, 5-aminosalicylate; IMM, immunomodulator; TNF $\alpha$, tumor necrosis factor $\alpha$.

\section{Perianal fistula outcome}

Of the 161 primary PFs diagnosed, 133 [82.6\%] were treated with antibiotics. Ninety-nine [61.5\%] patients underwent surgery for a PF and the surgery rate was similar in the three eras [55.9, 63.6 and 58.7\%, $p=0.71$, respectively]. Thirty-four [63.0\%] patients with a low PF were operated [all fistulotomy] and 32 [88.9\%] patients with a high PF required surgery.

Adjustments to CD maintenance therapy upon PF diagnosis were made in 46 of the 112 patients who developed a PF during the course of the disease [41.1\%], adjustments being more common in more recently diagnosed patients: $27.9 \%$ in the $1991-1998$ era versus $47.7 \%$ in the $1999-2005$ era versus $52.0 \%$ in the $2006-2011$ era, $p=0.04$. After PF 
diagnosis, an increasing number of CD patients were on anti-TNFa therapy [the 1991-1998 era: $18.6 \%$, the $1999-2005$ era: $34.1 \%$, the $2006-2011$ era: $54.0 \%, p<0.01]$, most [69.5\%] in combination with immunomodulator therapy [Figure 8.2].

In total, 58 [36.0\%] patients developed a PF recurrence. The cumulative probability of a PF recurrence was $10.7 \% 1$ year, $25.7 \% 5$ years and $36.7 \% 10$ years after the diagnosis of the primary PF. The median duration between the primary PF and recurrent PF was 2.9 [IQR 0.9-9.0] years. The cumulative 5-year recurrence rate was not found to be significantly different between eras: 19.5\% [the 1991-1998 era] versus 25.5\% [the 1999-2005 era] versus $33.1 \%$ [the 2006-2011 era], $p=0.22$ [adjusted for sex and disease location] [Figure 8.1B and Table 8.3]. The cumulative 5-year recurrence rate was found to be higher in patients who required surgery for their primary PF than in patients who were treated medically [33.0 vs. $13.8 \%$, HR: $4.08 ;$; $95 \%$ Cl: 1.98-8.38].

\section{Rectovaginal fistula incidence}

In total, 17 of the 728 [2.3\%] female CD patients ever had an RVF. Two [11.8\%] patients already had an RVF before CD diagnosis, with a time to CD diagnosis of 0.2 and 6.7 years. The other 15 RVFs developed during the course of disease at a median of 4.7 [IQR: 1.3-8.1] years after $\mathrm{CD}$ diagnosis. The overall cumulative probability of developing an RVF was $0.7 \%$ after 1 year, $1.7 \%$ after 5 years and $3.1 \%$ after 10 years. For these analyses, eras 1999 2005 and 2006-2011 were combined and subsequently compared with the 1991-1998 era because of the small number of events $[n=17]$. The cumulative 10 -year probability of developing an RVF was significantly lower in the 1999-2011 era than in the 1991-1998 era [ 1.7 vs. $5.7 \%$, HR: $0.28 ; 95 \% \mathrm{Cl}$ : $0.10-0.78$, respectively; see Figure 8.3$]$. Because of the limited number of events, only univariable analyses could be carried out to assess the risk factors for developing an RVF. No significant associations were found with age $[p=0.11]$, disease location $[p=0.47$ ] or disease behavior $[p=0.16]$.

Seven of the 10 [70.0\%] patients in the 1991-1998 era with an RVF who developed during the course of the disease had been exposed previously to immunomodulator or antiTNF $\alpha$ therapy compared with three of the five [60.0\%] patients in the 1999-2011 era. 
A

Cumulative probability of developing rectovaginal fistula

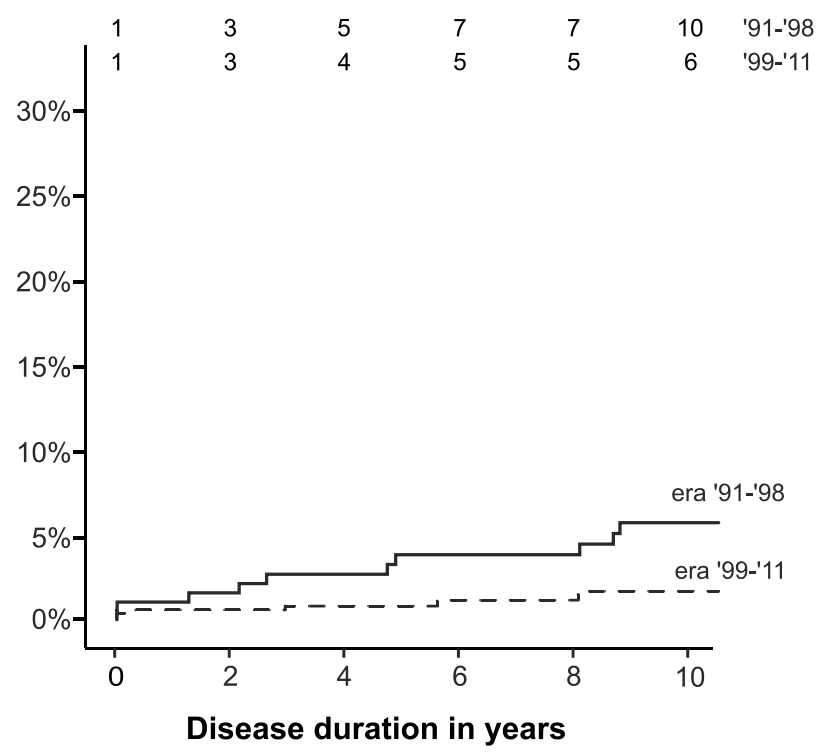

$\begin{array}{llllllll}\text { N at risk } & 195 & 181 & 172 & 164 & 155 & 145 & \text { '91-'98 } \\ \text { N at risk } & 533 & 482 & 372 & 268 & 195 & 114 & \text { '99-'11 }\end{array}$

Figure 8.3. Kaplan-Meier survival curve showing the cumulative probability of developing a rectovaginal fistula 


\section{Discussion}

In this population-based cohort study, we observed a stable cumulative 5-year PF risk of $11.6 \%$ over the past two decades, whereas the cumulative 5 -year RVF risk decreased from 5.7\% in patients diagnosed between 1991 and 1998 to $1.7 \%$ in patients diagnosed between 1999 and 2011. Over time, more patients are exposed to immunomodulator or anti-TNF $\alpha$ therapy; also before PF diagnosis, and an increasing number of patients received anti-TNF $\alpha$ therapy after fistula diagnosis. Patients with colon involvement had a higher risk of PF and older patients had a lower risk of developing a PF. More than half of the patients [61.5\%] required a surgical intervention and the surgery rate remained stable over time. The cumulative 5-year risk of recurrence of PF was $25.7 \%$, which was also stable over the past 20 years.

The present study is the first to study the evolution of the PF incidence in a large population-based cohort of CD patients in an era in which the medical management of CD and CD-related PFs changed significantly. Overall, a PF incidence of 13.9\% was observed, which is very similar to the rates observed in other population-based cohorts, such as Stockholm County [13.7\% $]^{6}$ and Manitoba [9.9-14.0\%]. ${ }^{7}$ Moreover, the cumulative 10-year PF risk of $15.8 \%$ observed in our cohort is in line with the cumulative 10-year risk observed in Minnesota [21\%] $]^{1}$ and Canterbury [16.9\%]. ${ }^{8}$ Over the past two decades, the cumulative PF risk was stable in our CD population. In parallel, marked changes have occurred in the medical management of $C D$ and the medical management of CD-related PFs, reflected by an increasing number of CD patients exposed to immunomodulators and anti-TNF $\alpha$ agents in general and before PF diagnosis. In a clinical trial that compared the effects of early azathioprine administration versus conventional management of $C D$, the authors found a lower risk of having active perianal lesions [14 vs. 27\%, $p=0.049$ ] and perianal surgery [3 vs. $13 \%, p=0.04]$ in the treatment arm that commenced immunomodulator therapy within 6 months after diagnosis compared with conventional management, respectively. ${ }^{18}$ Hence, it could be expected that the incidence of PF is lower in more recent eras, given that the number of patients who are exposed early to immunomodulators is increasing. However, the results of the present study do not confirm these findings. A possible explanation for this discrepancy may reside in the fact that, even in the most recent era, only $40.7 \%$ of the CD patients in the IBDSL cohort were exposed to immunomodulators within 6 months after diagnosis [data not shown]. Consequently, a potential beneficial effect of immunomodulator therapy on fistula development may therefore be not present at the population level. Ultimately, early azathioprine use may prevent the development of PF and PF surgery, but our findings support the conduction of future studies that should look at the number needed to treat early with immunomodulator therapy to prevent one PF. These findings should then be considered in the 
clinical discussion on balancing the risks and benefits of early and longstanding immunomodulator therapy.

The PF recurrence rate was $36.7 \%$ within 10 years after the primary PF. Cohort studies evaluating PF recurrences are scarce and definitions vary, not allowing a direct comparison of recurrence rates. Schwartz et al. ${ }^{1}$ observed a recurrence rate of $33 \%$ in CD patients diagnosed between 1970 and 1993 at a median time of 2.8 years after the primary PF. In a referral hospital setting, $6 \%$ of simple PF persisted and $32 \%$ relapsed after a median of 30 months, whereas 30\% of the complex PF persisted and 23\% relapsed after a median of 11 months. ${ }^{19}$ In line with previously discussed changes in medical management, we observed that, over time, an increasing number of CD patients were on immunomodulator or anti-TNF $\alpha$ therapy after the diagnosis of the primary PF, in line with current European and Dutch guidelines. ${ }^{20-21}$ It is noteworthy that more than half [54.0\%] of the most recently diagnosed CD patients were on anti-TNF $\alpha$ therapy after PF diagnosis, either as monotherapy or as combination therapy with immunomodulators. We found no significant differences in the PF recurrence rate between the three eras, indicating that changes in the medical management of PF seemed not to have resulted in fewer PF recurrences in general. However, we must acknowledge that the implementation of MRI and endoanal ultrasound in the followup of CD and PF, in particular, may have had impact on the reported recurrence rate as subclinical PFs may have been more often detected in more recent eras.

Next to changes in medical therapy, surgical techniques for high PFs have also changed over the past two decades, whereas treatment of low PFs has remained unchanged [fistulotomy]. For a long time, the seton and mucosal advancement flap were the most used techniques to treat high PFs. In addition to these classic surgical treatment options, several other techniques have been used over the years in our area, such as fibrin glue ${ }^{22-23}$, fistula plugs $^{23}$, ligation of the intersphincteric fistula tract $^{24}$ and, more recently, endoscopic techniques ${ }^{25}$ and the local injection of stem cells ${ }^{26}$ or platelet-rich plasma ${ }^{27}$ and variations on all these treatment options. ${ }^{28}$ Regrettably, we could not obtain detailed information on the specific surgical technique used in operated patients from our cohort. However, the number of patients treated with these new techniques is limited, and as such, the outcome of high PFs from the present study can mainly be interpreted as the outcome after seton and mucosal advancement flap interventions. Ultimately, the stable recurrence rate and the stable high surgery rate observed in this study underline the importance of developing new surgical procedures and the need for a direct comparison of current surgical treatment options.

The prevalence of RVFs in the IBDSL cohort was rather low [2.3\% in female CD patients]. Nevertheless, we found that the incidence of RVFs was significantly lower in the second decade of our cohort. The reason for this decrease is unclear. Further elaboration on this finding is warranted, particularly because a different time trend was found for PFs. 
Unfortunately, the low number of RVFs in the present study impeded further elaboration on this finding. As this is the first study that looked into the time trend of RVF incidence, future studies should confirm our observation.

Risk factors for the development of PFs have been reported in several cohort studies. ${ }^{1,6,7,9}$ In line with these studies, we observed that an older age at CD diagnosis was associated with a lower risk of developing PFs, whereas colonic disease involvement was found to be associated with an increased risk of developing PFs. We did not find sex to be related to the development of a PF, which may be related to the fact that we analyzed RVFs separately.

The strengths of the present study are its population-based nature, the long followup of patients and the long period of patient inclusion. This allowed us to study time trends in the PF and RVF incidences in an unselected, representative population of CD patients. Some limitations have to be addressed. First, the retrospective identification of the fistulas on the basis of medical files might have resulted in an under-representation of the true incidence of fistula, especially in the first era, in which imaging was not as commonly used in the diagnosis and follow-up of PFs and RVFs as it is nowadays. As a result, a theoretical decrease in the incidence of fistula over time might have been missed because of a parallel increase in the use of [new] diagnostic procedures. Second, the lack of detailed data on the specific surgical procedure did not allow a direct comparison of surgical techniques, which would have been of interest, considering the lack of comparative trials on this subject.

In conclusion, PFs are still common nowadays and the recurrence rate remains high, despite changes in treatment options and strategies for $C D$ and $C D$-related fistulas over the past two decades. RVFs occur less frequently in CD patients and its incidence has decreased over time. The findings of the present study underline the lasting importance of improving medical and surgical treatment strategies for these invalidating conditions. 


\section{References}

1. Schwartz DA, Loftus EV, Jr., Tremaine WJ, et al. The natural history of fistulizing crohn's disease in olmsted county, minnesota. Gastroenterology 2002;122:875-80.

2. Lakatos PL, Golovics PA, David G, et al. Has there been a change in the natural history of crohn's disease? Surgical rates and medical management in a population-based inception cohort from western hungary between 1977-2009. Am J Gastroentero/ 2012;107:579-88.

3. Lapidus A, Bernell O, Hellers G, Lofberg R. Clinical course of colorectal crohn's disease: A 35-year follow-up study of 507 patients. Gastroenterology 1998;114:1151-60.

4. Sandborn WJ, Fazio VW, Feagan BG, Hanauer SB, American Gastroenterological Association Clinical Practice C. Aga technical review on perianal crohn's disease. Gastroenterology 2003;125:1508-30.

5. Andersson P, Olaison G, Bendtsen P, Myrelid P, Sjodahl R. Health related quality of life in crohn's proctocolitis does not differ from a general population when in remission. Colorectal Dis 2003;5:56-62.

6. Lapidus A. Crohn's disease in stockholm county during 1990-2001: An epidemiological update. World J Gastroentero/2006;12:75-81.

7. Tang LY, Rawsthorne P, Bernstein CN. Are perineal and luminal fistulas associated in Crohn's disease? A population-based study. Clin Gastroenterol Hepato/2006;4:1130-4.

8. Eglinton TW, Barclay ML, Gearry RB, Frizelle FA. The spectrum of perianal crohn's disease in a population-based cohort. Dis Colon Rectum 2012;55:7737.

9. Hellers G, Bergstrand O, Ewerth S, Holmstrom B. Occurrence and outcome after primary treatment of anal fistulae in crohn's disease. Gut 1980;21:525-7.

10. Gecse KB, Sebastian S, Hertogh G, et al. Results of the fifth scientific workshop of the ECCO [ii]: Clinical aspects of perianal fistulising crohn's disease-the unmet needs. J Crohns Colitis 2016.

11. van den Heuvel TR, Jonkers DM, Jeuring SF, et al. Cohort profile: The inflammatory bowel disease South Limburg cohort [IBDSL]. Int J Epidemiol 2015.

12. Romberg-Camps MJ, Dagnelie PC, Kester AD, et al. Influence of phenotype at diagnosis and of other potential prognostic factors on the course of inflammatory bowel disease. Am J Gastroenterol 2009;104:371-83.

13. Gottgens KW, Smeets RR, Stassen LP, Beets G, Breukink SO. Systematic review and meta-analysis of surgical interventions for high cryptoglandular perianal fistula. Int J Colorectal Dis 2015;30:583-93

14. Parks AG, Gordon PH, Hardcastle JD. A classification of fistula-in-ano. Br J Surg 1976;63:1-12.

15. Silverberg MS, Satsangi J, Ahmad T, et al. Toward an integrated clinical, molecular and serological classification of inflammatory bowel disease: Report of a working party of the 2005 montreal world congress of gastroenterology. Can $J$ Gastroentero/2005;19 Suppl A:5A36A.

16. Satsangi J, Silverberg MS, Vermeire S, Colombel JF. The montreal classification of inflammatory bowel disease: Controversies, consensus, and implications. Gut 2006;55:749-53.

17. Gasche C, Scholmerich J, Brynskov J, et al. A simple classification of crohn's disease: Report of the working party for the world congresses of gastroenterology, vienna 1998. Inflamm Bowel Dis 2000;6:8-15. 
18. Cosnes J, Bourrier A, Laharie D, et al. Early administration of azathioprine vs conventional management of crohn's disease: A randomized controlled trial. Gastroenterology 2013;145:758-65 e2; quiz e14-5.

19. Bell SJ, Williams AB, Wiesel $P$, et al. The clinical course of fistulating crohn's disease. Aliment Pharmacol Ther 2003; 17:1145-51.

20. Van Assche G, Dignass A, Reinisch W, et al. The second european evidence-based consensus on the diagnosis and management of crohn's disease: Special situations. J Crohns Colitis 2010;4:63101.

21. van Bodegraven $A A$, van Everdingen $\mathrm{JJ}$, Dijkstra G, et al. [guideline 'diagnosis and treatment of inflammatory bowel disease in adults'. I. Diagnosis and treatment]. Ned Tijdschr Geneeskd 2010;154:A1899.

22. Abel ME, Chiu YS, Russell TR, Volpe PA. Autologous fibrin glue in the treatment of rectovaginal and complex fistulas. Dis Colon Rectum 1993;36:447-9.

23. Johnson EK, Gaw JU, Armstrong DN. Efficacy of anal fistula plug vs. Fibrin glue in closure of anorectal fistulas. Dis Colon Rectum 2006;49:371-6.

24. Rojanasakul A, Pattanaarun J, Sahakitrungruang C, Tantiphlachiva K. Total anal sphincter saving technique for fistula-in-ano; the ligation of intersphincteric fistula tract. J Med Assoc Thai 2007;90:581-6.

25. Meinero P, Mori L. Video-assisted anal fistula treatment [vaaft]: A novel sphincter-saving procedure for treating complex anal fistulas. Tech Coloproctol 2011;15:417-22.

26. Garcia-Olmo D, Herreros D, Pascual I, et al. Expanded adipose-derived stem cells for the treatment of complex perianal fistula: A phase ii clinical trial. Dis Colon Rectum 2009;52:79-86.

27. van der Hagen SJ, Baeten CG, Soeters PB, van Gemert WG. Autologous platelet-derived growth factors [plateletrich plasma] as an adjunct to mucosal advancement flap in high cryptoglandular perianal fistulae: A pilot study. Colorectal Dis 2011;13:215-8.

28. Han JG, Yi BQ, Wang ZJ, et al. Ligation of the intersphincteric fistula tract plus a bioprosthetic anal fistula plug [lift-plug]: A new technique for fistula-in-ano. Colorectal Dis 2013;15:582-6 



\section{Abstract}

\section{Background}

Clinical trials and meta analyses have proven the efficacy of infliximab [IFX] and adalimumab [ADA] for induction and maintenance of remission in Crohn's disease [CD]. The aim of the present study was to study the long-term and comparative effectiveness of these agents in a real-world population-based cohort.

\section{Methods}

All CD patients from the IBD South Limburg cohort who used IFX [ $n=295]$ or ADA [ $n=84]$ as first-line anti-tumor necrosis factor $\alpha$ [anti-TNF $\alpha$ ] therapy were included. Patients were diagnosed between 1991 and 2011 and followed until 2014. The long-term corticosteroidfree response [CSFR] rate of anti-TNF $\alpha$ therapy was assessed by using Kaplan-Meier survival statistics, adjusted for confounders by using multivariable Cox regression modelling. Propensity score matching was used to compare outcomes between IFX and ADA and between monotherapy and combination therapy.

\section{Results}

In total, $50.1 \%$ of IFX patients and $60.9 \%$ of ADA patients had durable CSFR after five years. In the propensity score matched analyses, no difference in the 5-year CSFR was found between agents [63.2\% vs. 60.5\%, $p=0.93$ ], nor between IFX monotherapy and combination therapy $[50.5 \%$ vs. $52.9 \%, p=0.98]$. After treatment discontinuation, $20.5 \%$ underwent surgery within one year and $44.9 \%$ restarted anti-TNFa therapy within five years. Subsequently, 32.9\% showed durable 5-year treatment response to the second agent.

\section{Conclusion}

In a real-world population-based cohort of CD patients, both IFX and ADA had durable response rates. We found no superiority of one agent over the other. Therefore, the choice for an anti-TNF $\alpha$ agent can be based on the preferred administration route and costs. 


\section{Introduction}

Crohn's disease is a chronic inflammatory disease of the intestinal tract with an unfavorable long-term outcome for many patients. Previous studies have shown that approximately half of the patients requires surgery and more than half develops stricturing or penetrating disease..$^{1-3}$ The introduction of anti-tumor necrosis factor $\alpha$ [anti-TNF $\alpha$ ] therapy in the late nineties was considered the start of a new era of CD management, given the different mechanism of action of these agents as compared to other IBD medication at that time.

Randomized controlled trials [RCTs] and meta analyses have proven the efficacy of infliximab [IFX) and adalimumab [ADA] and resulted in their registration in the Netherlands in 1999 [IFX] and 2007 [ADA] for moderate to severe CD. In the ACCENT-I trial on the efficacy of IFX as maintenance therapy, 38\% of the primary responders maintained clinical response and 29\% were in corticosteroid-free clinical remission at week 54. ${ }^{4}$ In the CLASSIC-II and CHARM trials on the effect of ADA as maintenance therapy, corresponding proportions were 52-65\% and 36-46\% at week 56. ${ }^{5-7}$ Currently, a similar efficacy is being ascribed to IFX and ADA, based on indirect comparisons in network analyses ${ }^{8}$, as head-to-head trials are lacking.

Data from the registration trials provided information on the efficacy of IFX and ADA [i.e. whether they provided direct health benefit ${ }^{9}$ ]. Additionally, it is important to have insight in their effectiveness [i.e. their effect in real-life clinical practice ${ }^{10}$ ], since the generalizability of trial results is limited. For example, study populations are often highly selected and intensely monitored as compared to real-world patient populations ${ }^{11}$ and treatment indication is in reallife affected by local policies, costs, reimbursement, and availability. Most previous real-world studies on anti-TNF $\alpha$ therapy effectiveness were performed in referral centres ${ }^{12-15}$ or made use of large administrative databases. ${ }^{16,17}$ Population-based cohorts have the advantage of reflecting on an unselected $\mathrm{IBD}$ population and have detailed data on patient and disease characteristics, required for addressing confounding.

Therefore, the aim of the present study was to assess the use and effectiveness of IFX and ADA in a large, population-based cohort of CD patients. We evaluated the long-term treatment outcomes of these agents and performed comparative effectiveness analyses between IFX and ADA and between anti-TNF $\alpha$ monotherapy and combination therapy. 


\section{Methods}

\section{Study population and data collection}

The IBD South Limburg [IBDSL] cohort is a large population-based cohort in the South Limburg area of the Netherlands. As from 1991, newly diagnosed IBD patients in the area are registered and their disease outcome is recorded via a thorough review of the medical records. A multi-faceted completeness check has shown that over 93\% of all eligible IBD patients in the South Limburg area is registered in the cohort. For detailed information on the design of the cohort, we refer to the cohort design paper. ${ }^{18}$ For this study, all 1162 CD patients registered in the cohort were eligible for inclusion. These patients were diagnosed between January $1^{\text {st }} 1991$ and July $1^{\text {st }} 2011$, and were followed until December 2014, migration, or death.

All data on patient and disease characteristics, including medication status, were directly retrieved from the medical records by trained physicians, using a standardized report form. Disease phenotype was classified in line with the Montreal consensus ${ }^{19}$ and assessed at time of CD diagnosis and at time of the initiation of anti-TNF $\alpha$ therapy. Cumulative corticosteroid use was defined as the total number of days on which corticosteroid therapy was used between CD diagnosis and start of anti-TNFa therapy. Regarding the outcome measures, hospitalization was defined as a hospital admission for CD-related symptoms or surgery. Elective admissions for endoscopy procedures or regular drug administration were not included. Surgery was defined as resection of part of the bowel for refractory intestinal inflammation or disease-related complications. Perianal fistula surgery was excluded. Combination therapy was defined as the concomitant use of an immunomodulator [azathioprine, mercaptopurine, or methotrexate] within three months after start of anti-TNF $\alpha$ therapy.

In the Netherlands, IFX was registered in 1999 and ADA in 2007. As from their availability, the European ECCO guideline ${ }^{20}$ and the derived Dutch CBO guideline ${ }^{21}$ have been advocated regarding therapy indication and usage. In the study period, there was no local, uniform consensus on the use of combination therapy, when to stop anti-TNFa therapy, or when to use IFX trough levels.

\section{Study endpoints and design}

The objectives of the study were [1] to assess the long-term effectiveness of IFX and ADA as first-line anti-TNF $\alpha$ agent, [2] to directly compare the effectiveness of IFX and ADA as firstline anti-TNF $\alpha$ agent and [3] to compare the effectiveness of anti-TNF $\alpha$ monotherapy and combination therapy in a real-world population-based cohort. 
The long-term effectiveness was assessed by determining the corticosteroid-free clinical response [CSFR], a previously used outcome parameter in observational studies. ${ }^{22}$ This parameter resembles the definition of corticosteroid-free remission, commonly used in clinical trials. Corticosteroid-free clinical response was defined as the situation in which anti-TNF $\alpha$ treatment was not discontinued because of primary or secondary loss of response, and no corticosteroid therapy, hospital admission, or surgery was required. Corticosteroid use at the start of anti-TNFa therapy was allowed, but had to be completely withdrawn within three months to be classified as 'anti-TNFa responder', in line with the definition of corticosteroid dependency. ${ }^{23}$ As secondary outcome measures, the risks of requiring corticosteroid therapy, hospitalization, and surgery after anti-TNF $\alpha$ discontinuation were individually studied.

The comparative effectiveness between IFX and ADA and between monotherapy and combination therapy was studied using the same outcome parameters.

\section{Statistical analyses}

Continuous data were presented as means with corresponding standard deviations [SD] or as medians with corresponding interquartile ranges [IQR], depending on the normality of the underlying distribution. For baseline characteristics, data were compared by independent Student's T-test [two categories] or One-way ANOVA [more than two categories] analyses in case of parametric data and by Mann-Whitney $U$ test [two categories] or Kruskal-Wallis test analyses [more than two categories] in case of non-parametric data.

Kaplan-Meier survival analyses were conducted to estimate the CSFR rate, as well as the need for corticosteroid therapy, hospitalization, surgery, and second-line anti-TNF $\alpha$ therapy, in order to adjust for differences in follow-up between patients. In the long-term effectiveness analyses, independent associations between patient and disease characteristics with the respective outcome measures were assessed by using a multivariable Cox regression model. Parameters included in the models were: age, sex, disease duration, disease phenotype, smoking status, cumulative corticosteroid use prior to the start of anti-TNFa therapy, year of first anti-TNFa prescription after 1999, and the type of anti-TNFa agent. All parameters were assessed at time of treatment start, except for smoking status, which was only available at time of $\mathrm{CD}$ diagnosis. In case not all parameters were allowed in a multivariable model due to a limited number of events [less than 10 times the number of parameters], only parameters with $p<0.10$ in univariable analyses were included. Results were expressed as hazard ratios $[\mathrm{HR}]$ with accompanying $95 \%$ confidence intervals $[95 \% \mathrm{Cl}]$. The proportional hazards assumption was tested for all parameters, by including time-dependent interaction terms to the model.

Propensity score matching was applied in the comparative effectiveness analyses. By using this approach, baseline parameters were balanced between the IFX and ADA group. 
It thereby diminished the risk of confounding resulting from non-random treatment allocation. A logistic regression model with ADA vs. IFX as outcome parameter was created to obtain a propensity score for every patient. All parameters from the aforementioned multivariable models were included, as well as the parameters: indication for anti-TNF $\alpha$ therapy, history of surgery, history of immunomodulator use, hospital, and start of anti-TNF $\alpha$ therapy during a hospital admission. Based on their propensity score, ADA patients were 1:1 matched to IFX patients with a caliper of 0.2 and allowing case replacement. After matching, all parameters were found to be well balanced, as reflected by the absence of parameters with a standardized mean difference of 0.25 or more.

Second, the effect of combination therapy on the outcome measures was studied. To adjust for the assumption that patients using combination therapy have more severe disease than patients on monotherapy, a similar propensity score modelling approach was employed. Based on the propensity score, patients on combination therapy were matched to patients on monotherapy and the aforementioned outcome parameters were subsequently compared between groups. Follow-up was censored at the time patients on combination therapy stopped the concomitant immunomodulator or patients on monotherapy started an immunomodulator.

In all analyses, two-sided p-values lower than 0.05 were considered statistically significant. All statistical analyses were conducted using IBM SPSS Statistics for Windows, Version 23.0. [IBM Corp. Armonk, NY, USA]. 


\section{Results}

\section{Patient population}

All 1162 CD patients from the IBDSL registry were included in the present study. Overall, the cumulative anti-TNF $\alpha$ exposure rate was 10.7\%,22.9\%, and 35.4\% at one, five, and ten years after $\mathrm{CD}$ diagnosis. More recently diagnosed patients were more often and earlier exposed to these biologicals, reflected by increases in the cumulative 1 - and 5-year exposure rate over time: $0 \%$ and $3.1 \%$ in patients diagnosed between 1991 and $1998,7.1 \%$ and $19.9 \%$ in patients diagnosed between 1999 and 2005, and $21.2 \%$ and $41.2 \%$ in patients diagnosed between 2006 and 2011, respectively.

In total, 295 patients received IFX and 84 patients ADA as first-line anti-TNF $\alpha$ therapy after a median of 2.3 years [IQR 0.6-7.2] and 3.3 years [IQR 1.1-7.2] after CD diagnosis, respectively. Baseline characteristics of CD patients on anti-TNF $\alpha$ therapy and CD patients who never used anti-TNF $\alpha$ therapy are outlined in Table 9.1. In a multivariable model, active smoking [aHR $1.31 ; 95 \% \mathrm{Cl} 1.06-1.62$ ], colonic [aHR 1.38; 95\% Cl 1.06-1.80] or ileocolonic [aHR 1.47; 95\% Cl 1.13-1.92] disease localization, perianal disease [aHR 1.93; $95 \% \mathrm{Cl} 1.43-2.60$ ], and upper $\mathrm{Gl}$ disease [aHR 1.93; 95\% Cl 1.40-2.68] at diagnosis were significantly and independently associated with a higher probability of receiving anti-TNFa treatment during disease course, whereas older age [>60, aHR 0.56; 95\% Cl 0.33-0.93] was associated with a lower probability.

The main indication for starting anti-TNFa therapy was refractory luminal disease [70.7\%]. Other indications included immunomodulator intolerance [12.4\%], perianal disease [11.3\%], penetrating disease [4.0\%], concomitant rheumatoid disease [1.1\%], and extraintestinal manifestations [0.5\%]. Therapy indication was not different between IFX and ADA $[p=0.23]$.

During anti-TNF $\alpha$ therapy, 131 patients [34.8\%] received treatment optimization. In 66 IFX users [22.4\%] the interval was reduced and in 42 patients [14.2\%] the dose was increased. For ADA, these numbers were 21 [25.0\%] and 2 [2.4\%], respectively. These optimizations were performed after a median of 0.9 years [IQR 0.5-1.9] after start of therapy. No difference in optimization frequency was observed between IFX [36.9\%] and ADA $[27.4 \%], p=0.12$. 
Table 9.1. Patient characteristics of Crohn's disease patients from the IBDSL cohort

\begin{tabular}{|c|c|c|c|c|c|}
\hline & & $\begin{array}{l}\text { IFX } \text { users }^{\mathrm{a}} \\
{[\mathrm{N}=295]}\end{array}$ & $\begin{array}{c}\text { ADA users }^{a} \\
{[\mathrm{~N}=84]}\end{array}$ & $\begin{array}{l}\text { anti-TNF } \alpha \\
\text { naive }\end{array}$ & \\
\hline Age in years & mean $[S D]$ & $33.6[14.0]$ & $33.3[12.0]$ & $39.7[16.6]$ & $p_{1}<0.01 / p_{2}=0.90$ \\
\hline Sex - male & $N[\%]$ & 91 [30.8] & 33 [39.3] & 310 [39.6] & $p_{1}=0.02 / p_{2}=0.15$ \\
\hline Current smoker & $N[\%]$ & 158 [58.3] & $46[56.1]$ & $290[45.3]$ & $p_{1}<0.01 / p_{2}=0.80$ \\
\hline $\begin{array}{l}\text { Disease duration } \\
\text { in years }\end{array}$ & median $[\mathrm{QQR}]$ & $9.0[5.3-13.8]$ & $7.0[4.6-10.6]$ & $7.2[3.6-12.4]$ & $p_{1}<0.01 / p_{2}<0.01$ \\
\hline $\begin{array}{l}\text { Time to first anti-TNF } \alpha \\
\text { administration in years }\end{array}$ & median $[\mathrm{QQR}]$ & $2.3[0.6-7.2]$ & $3.3[1.1-7.2]$ & - & $p_{1}=-/ p_{2}=0.17$ \\
\hline Location $^{a, b}$ & & & & & $p_{1}=0.02 / p_{2}=0.12$ \\
\hline L1, ileum & $N[\%]$ & $106[35.9]$ & $40[47.6]$ & $354[45.2]$ & \\
\hline L2, colon & $N[\%]$ & $102[34.6]$ & 20 [23.8] & 249 [31.8] & \\
\hline L3, ileocolon & $N[\%]$ & $84[28.5]$ & $22[26.2]$ & $161[20.6]$ & \\
\hline L4, only upper GI & $N[\%]$ & $3[1.0]$ & $2[2.4]$ & $19[2.4]$ & \\
\hline$\llcorner 4$, modifier & $N[\%]$ & 36 [12.2] & 19 [22.6] & $69[8.8]$ & \\
\hline Behavior $^{a}$ & & & & & $p_{1}=0.10 / p_{2}=0.51$ \\
\hline B1, inflammatory & $N[\%]$ & 224 [75.9] & 59 [70.2] & $617[78.8]$ & \\
\hline B2, stricturing & $N[\%]$ & $51[17.3]$ & 19 [22.6] & $107[13.7]$ & \\
\hline B3, penetrating & $N[\%]$ & $20[6.8]$ & $6[7.1]$ & $59[7.5]$ & \\
\hline P, perianal disease & $N[\%]$ & $49[16.6]$ & $8[9.5]$ & $37[4.7]$ & $p_{1}<0.01 / p_{2}=0.12$ \\
\hline $\begin{array}{l}\text { Concomitant } \\
\text { immunomodulator thera }\end{array}$ & & & & & \\
\hline $\begin{array}{l}\text { at start } \\
\text { anti-TNF } \alpha \text { therapy }\end{array}$ & $N[\%]$ & $139[47.1]$ & $26[31.0]$ & & $p_{1}=-/ p_{2}<0.01$ \\
\hline $\begin{array}{l}\text { discontinuation } \\
\text { during follow-up }\end{array}$ & $N[\%]$ & 65 [46.8] & 9 [34.6] & & $p_{1}=-/ p_{2}=0.29$ \\
\hline $\begin{array}{l}\text { Bowel surgery prior to } \\
\text { anti-TNF } \alpha \text { therapy }\end{array}$ & $N[\%]$ & $44[14.9[$ & $19[22.3]$ & & $p_{1}=-/ p_{2}=0.10$ \\
\hline \multicolumn{4}{|c|}{ anti-TNF $\alpha$, anti tumor necrosis factor $\alpha$} & & \\
\hline \multicolumn{6}{|l|}{${ }^{b}$ at diagnosis } \\
\hline \multicolumn{6}{|c|}{${ }^{\mathrm{C}}$ smoking status could not be retrieved in 169 cases } \\
\hline \multicolumn{6}{|c|}{ d disease phenotype according to the Montreal consensus } \\
\hline \multicolumn{6}{|c|}{$p_{1} p$-value of the test between anti-TNF $\alpha$ users and anti-TNF $\alpha$ naïve patients } \\
\hline \multicolumn{6}{|c|}{$p_{2} p$-value of the test between infliximab and adalimumab users } \\
\hline
\end{tabular}




\section{Corticosteroid-free response}

Of the 295 CD patients who started with IFX as first-line anti-TNFa agent, 67.2\% had CSFR during one year, $57.6 \%$ during two years, $50.1 \%$ during five years, and $38.8 \%$ during ten years of follow-up [Figure 9.1A]. Fifty-eight [19.7\%] patients commenced IFX therapy in an in-hospital setting. IFX patients who achieved CSFR within six months, had a chance of $67.1 \%$ of showing sustained CSFR over the subsequent five years. For ADA, 76.9\% had CSFR for one year, $63.0 \%$ for two years, and $60.9 \%$ for five years [Figure 9.1A]. Only one [1.2\%] patient started ADA therapy during hospitalization. The cumulative 5-year CSFR rate was $70.1 \%$ in ADA patients who achieved CSFR within six months.

A

\section{UNADJUSTED}

Cumulative probability of corticosteroid-free response

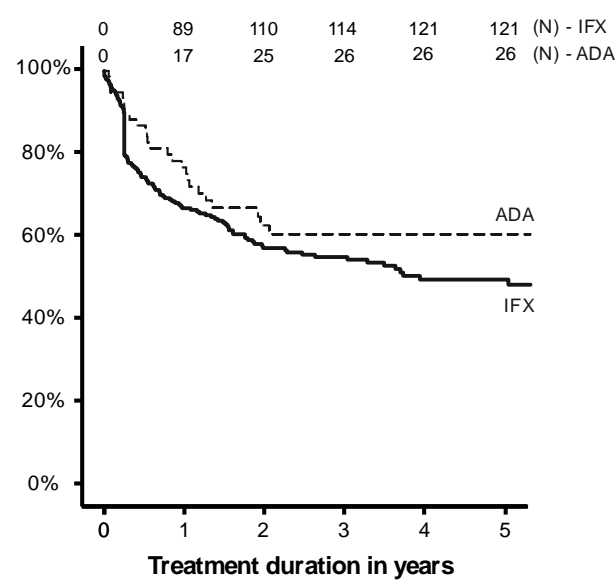

$\begin{array}{llllllll}\text { Nat risk } & 295 & 164 & 119 & 87 & 53 & 41 & \text { IFX }\end{array}$

$\begin{array}{llllllll}\mathrm{N} \text { at risk } & 84 & 50 & 30 & 16 & 7 & 1 & \text { ADA }\end{array}$

\section{B}

PROPENSITY SCORE MATCHED

Cumulative probability of corticosteroid-free response

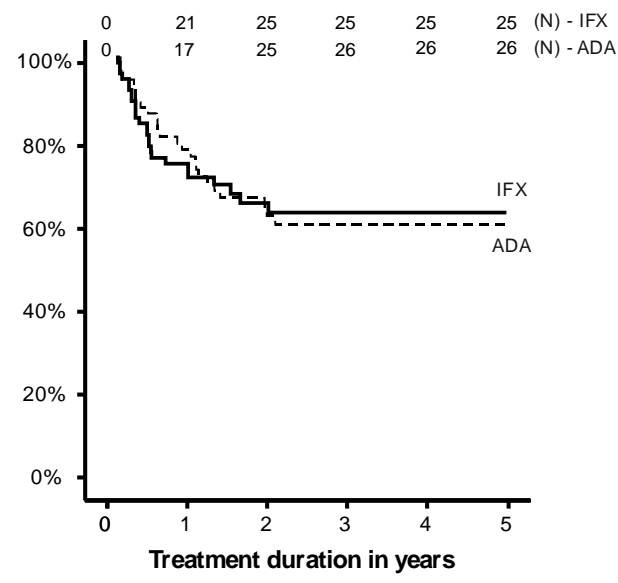

$\begin{array}{lllllllll}\text { Natrisk } & 80 & 44 & 28 & 18 & 8 & 6 & \text { IFX }\end{array}$

$\begin{array}{llllllll}\mathrm{N} \text { at risk } & 80 & 49 & 30 & 16 & 7 & 1 & \text { ADA }\end{array}$

Figure 9.1. Long-term cumulative corticosteroid-free response in patients on infliximab and adalimumab in the original cohort $[\mathrm{A}]$ and in the propensity score matched cohort [B]

IFX, infliximab. ADA, adalimumab. N, number of patients.

By using propensity score modelling, 80 IFX patients could be matched to 80 ADA patients. The CSFR rate of the propensity score matched cohort is shown in Figure 9.1B. In this matched cohort, the CSFR rate was not different between IFX and ADA users [5-year 63.2\% vs. $60.5 \%$, HR $1.02 ; 95 \% \mathrm{Cl} 0.63-1.66]$.

Clinical factors associated with sustained CSFR were determined in a multivariable Cox regression model, in which IFX and ADA patients were combined [see Table 9.2]. 
Table 9.2. Parameters associated with corticosteroid-free response

\begin{tabular}{|c|c|c|c|}
\hline & $\begin{array}{l}\text { Cumulative 5-year } \\
\text { probability of CSFR }\end{array}$ & $\begin{array}{l}\text { Unadjusted } \\
\text { Odds Ratio } \\
\text { OR [95\%Cl] }\end{array}$ & $\begin{array}{c}\text { Adjusted } \\
\text { Odds Ratio } \\
\text { OR }[95 \% \mathrm{Cl}]\end{array}$ \\
\hline \multicolumn{4}{|l|}{$\operatorname{Age}^{a}$} \\
\hline $18-40$ years & $52.2 \%$ & ref & ref \\
\hline 41-60 years & $45.5 \%$ & $0.81[0.58-1.13]$ & 0.69 [0.48-0.99] \\
\hline 61 years and older & $77.4 \%$ & $1.49[0.72-3.07]$ & $1.46[0.57-3.75]$ \\
\hline \multicolumn{4}{|l|}{ Sex } \\
\hline male & $49.3 \%$ & ref & ref \\
\hline female & $52.7 \%$ & 1.44 [1.04-1.99] & $1.70[1.21-2.40]$ \\
\hline \multicolumn{4}{|l|}{ Current smoker $^{\mathrm{b}}$} \\
\hline no & $55.6 \%$ & ref & ref \\
\hline yes & $48.5 \%$ & 0.77 [0.55-1.09] & $0.88[0.61-1.27]$ \\
\hline \multicolumn{4}{|l|}{ Disease location ${ }^{a}$} \\
\hline L1, ileum & $53.6 \%$ & ref & ref \\
\hline L2, colon & $52.0 \%$ & $0.91[0.59-1.41]$ & $0.88[0.52-1.50]$ \\
\hline L3, ileocolon & $50.8 \%$ & $0.90[0.61-1.32]$ & $0.88[0.57-1.36]$ \\
\hline$\llcorner 4$, only upper GI & - & - & - \\
\hline \multicolumn{4}{|l|}{ Behavior ${ }^{a}$} \\
\hline B1, inflammatory & $56.9 \%$ & ref & ref \\
\hline B2, stricturing & $39.6 \%$ & $0.72[0.50-1.02]$ & 0.60 [0.38-0.93] \\
\hline B3, penetrating & $54.1 \%$ & $0.94[0.56-1.56]$ & $1.01[0.57-1.80]$ \\
\hline \multicolumn{4}{|l|}{ Perianal disease ${ }^{a}(+p)$} \\
\hline no & $49.7 \%$ & ref & ref \\
\hline yes & $58.3 \%$ & $1.30[0.88-1.92]$ & $1.43[0.91-2.24]$ \\
\hline \multicolumn{4}{|l|}{ Upper Gl location ${ }^{\mathrm{a}}(+\angle 4)$} \\
\hline no & $53.1 \%$ & ref & ref \\
\hline yes & $47.1 \%$ & $0.95[0.65-1.38]$ & $1.10[0.71-1.72]$ \\
\hline \multicolumn{4}{|l|}{ anti-TNF $\alpha$ agent } \\
\hline infliximab & $50.1 \%$ & ref & ref \\
\hline adalimumab & $60.9 \%$ & $1.31[0.86-2.00]$ & $1.11[0.70-1.77]$ \\
\hline \multicolumn{4}{|l|}{ Start of anti-TNF $\alpha$ therapy during hospitalization } \\
\hline no & $53.2 \%$ & ref & ref \\
\hline yes & $37.6 \%$ & $0.64[0.42-0.95]$ & $0.61[0.38-0.97]$ \\
\hline & \multicolumn{3}{|c|}{ - per month of corticosteroid use } \\
\hline Disease duration at start anti-TNF $\alpha$ therapy - & & $1.01[0.98-1.05]$ & $1.04[0.99-1.10]$ \\
\hline \multicolumn{4}{|l|}{ per year } \\
\hline \multicolumn{4}{|l|}{ after biological availability ${ }^{c}$ - per year } \\
\hline \multicolumn{4}{|l|}{$\begin{array}{l}\text { anti-TNF } \alpha \text {, anti tumor necrosis factor } \alpha \\
\text { a at time of start anti-TNF } \alpha \text { therapy }\end{array}$} \\
\hline \multicolumn{4}{|l|}{ a at time of start anti-TNF $\alpha$ therapy } \\
\hline b at time of Crohn's disease diagnosis & & & \\
\hline${ }^{\mathrm{c}}$ year of biological availability in the Netherl & 999 & & \\
\hline
\end{tabular}


Commencing anti-TNFa therapy during hospitalization was associated with a lower CSFR rate [adjusted HR 0.61; 95\% 0.38-0.97], and we found the CSFR rate to be independently related to the calendar year of starting anti-TNF $\alpha$ therapy, in the way that the CSFR rate was higher in patients who more recently started therapy [adjusted HR 1.08; 95\%Cl 1.02-1.14 per year as from 1999].

\section{Anti-TNF $\alpha$ therapy discontinuation and outcome}

In total, 169 IFX and 36 ADA patients discontinued treatment during follow-up. The corresponding cumulative discontinuation rates are $26.9 \%, 41.1 \%$, and $57.7 \%$ after one, two, and five years, respectively, for IFX and $21.8 \%, 35.6 \%$, and $68.4 \%$, respectively, for ADA. In the propensity score analysis, no difference between IFX and ADA was observed herein [5year, $55.1 \%$ vs. $69.2 \%, \mathrm{HR} 1.01 ; 95 \% \mathrm{Cl} 0.66-1.56, p=0.95]$. Treatment was stopped because of loss of response [38.1\%], quiescent disease [18.8\%], side effects [31.7\%], or because of other reasons [11.4\%], such as pregnancy, infection risk, or concomitant malignant diseases. Common side effects resulting in treatment discontinuation included infusion or injection reactions [28.1\%], skin rashes [14.1\%], joint complaints [14.1\%], delayed hypersensitivity reactions [12.5\%], and other diverse side effects [31.2\%], such as malaise, headache, and nausea.

The Kaplan-Meier curves illustrating the disease course after treatment cessation are presented in Figure 9.2. In the first year after discontinuation, $17.8 \%$ required corticosteroids, $22.5 \%$ required hospitalization, and $20.5 \%$ underwent surgery. Twenty-five [12.3\%] patients started another anti-TNFa agent [86.8\%] or restarted with the same agent [13.2\%] within one year after therapy discontinuation, and nearly half [44.9\%] restarted antiTNF $\alpha$ therapy within five years. Subsequently, the second anti-TNF $\alpha$ treatment was discontinued in $67.1 \%$ within five years, because of loss of response [40.0\%], quiescent disease [20.0\%], side effects [7.3\%], or other reasons [32.7\%], mainly pregnancy. The risk of discontinuing the second anti-TNFa was not different between patients who failed the first anti-TNF $\alpha$ agent [64.1\%] and patients who stopped treatment because of side effects [69.6\%] or quiescent disease [70.3\%], $p=0.35$. 
A

Corticosteroid-free survival

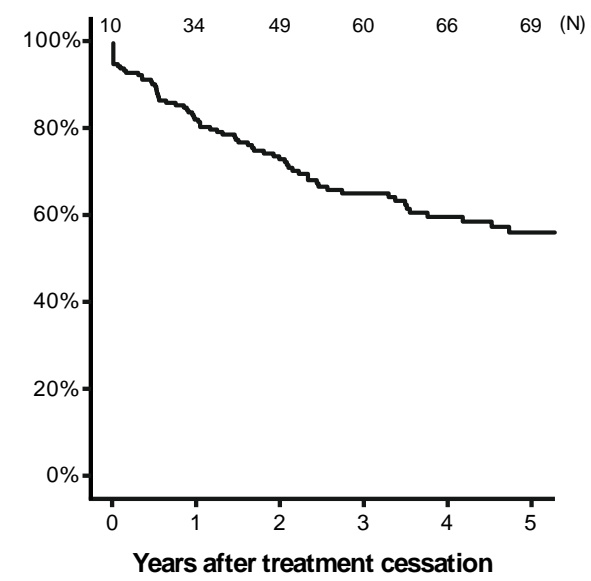

$\begin{array}{lllllll}\text { No at risk } & 205 & 146 & 110 & 77 & 58 & 39\end{array}$

C

Surgery-free survival

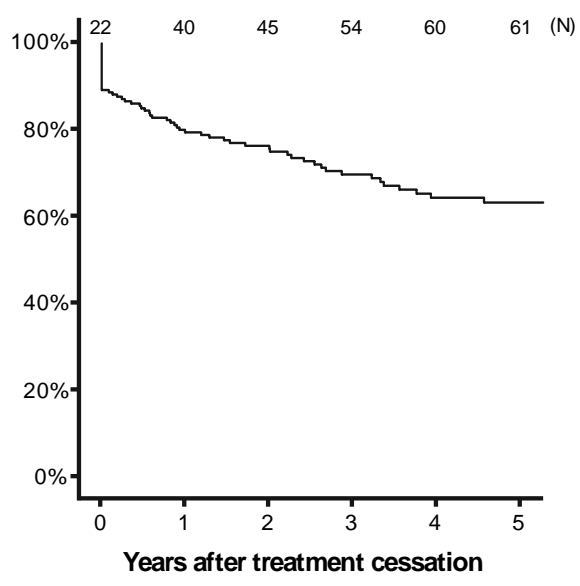

$\begin{array}{lllllll}\text { No at risk } & 205 & 139 & 113 & 84 & 66 & 52\end{array}$
B

Hospitalisation-free survival

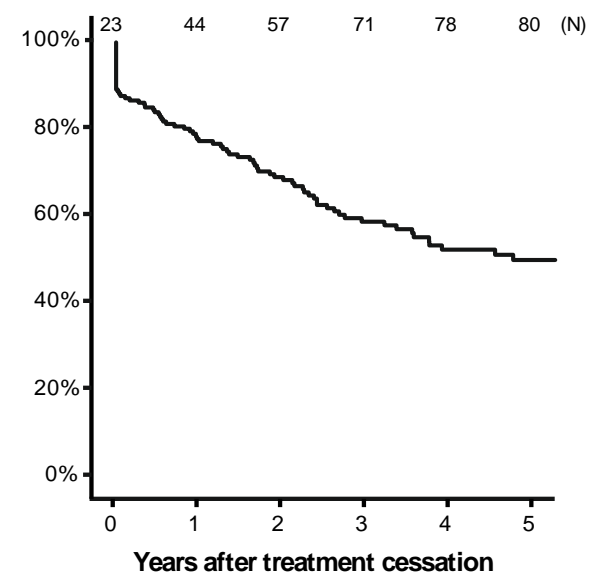

$\begin{array}{lllllll}\text { No at risk } & 205 & 136 & 103 & 72 & 52 & 41\end{array}$

D Cumulative exposure to a second anti-TNF $\alpha$ agent

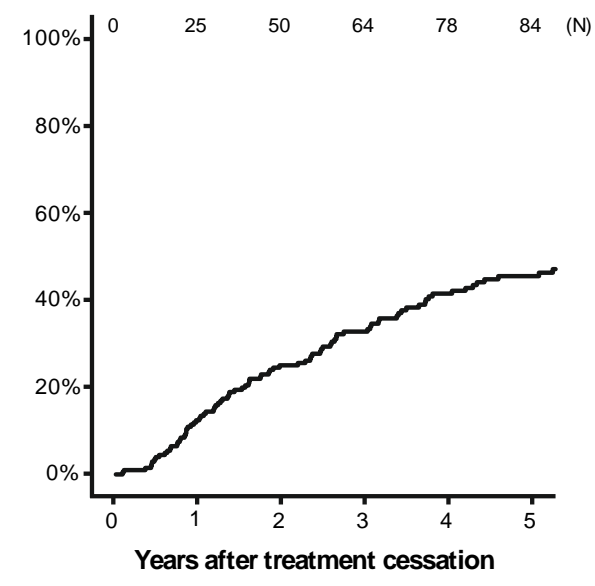

$\begin{array}{lllllll}\text { No at risk } & 205 & 179 & 148 & 113 & 93 & 72\end{array}$

Figure 9.2. Long-term outcome after anti-TNF $\alpha$ therapy cessation in terms of corticosteroid exposure [A], hospitalization [B], surgery [C], and the start of a second anti-TNFa agent [D] 


\section{Combination therapy}

Concomitant immunomodulator therapy was used by $57.6 \%$ of the IFX and $35.7 \%$ of the ADA patients. During the course of anti-TNFa therapy, the immunomodulator was discontinued in $44.7 \%$ and $40.0 \%$ of the combination therapy users, while an immunomodulator was added in $15.2 \%$ and $9.3 \%$ of the monotherapy users, respectively.

In total, 197 CD patients on combination therapy could be matched to 197 cases on anti-TNF $\alpha$ monotherapy. The CSFR rate was not significantly different between combination and monotherapy [5-year: $46.5 \%$ vs. $51.4 \%$, HR $1.06 ; 95 \% \mathrm{Cl} 0.77-1.46$ ], nor were the 5 year risks of requiring hospitalization [41.1\% vs. $28.8 \%$, HR $1.04 ; 95 \% \mathrm{Cl} 0.66-1.54]$ or surgery [ $27.9 \%$ vs. $27.1 \%$, HR $0.85 ; 95 \% \mathrm{Cl} 0.50-1.44]$. If only IFX patients were considered [165 CD patients combination therapy matched to 165 patients on IFX monotherapy], also no significant differences were observed in the CSFR rate [5-year: $50.5 \%$ vs $52.9 \%, H R$ 1.01; $95 \% \mathrm{Cl} 0.70-1.44$ ], the risk of hospitalization [5-year: $36.9 \%$ vs. $21.6 \%, \mathrm{HR} 1.26 ; 95 \% \mathrm{Cl}$ $0.75-2.12$ ], nor in the risk of surgery [5-year: $25.3 \%$ vs. $29.0 \%, \mathrm{HR} 1.16 ; 95 \% \mathrm{Cl} 0.60-2.23$ ] [Table 9.S1]. 


\section{Discussion}

In this real-world, population-based study, we found that anti-TNF $\alpha$ therapy is increasingly being used in $C D$ management and that more than half of the patients show durable corticosteroid-free response to treatment. Retreatment after failure was successful in onethird of the cases. No differences between IFX and ADA were observed in terms of achieving corticosteroid-free response, nor in the risks of hospitalization or surgery. We also found no difference in the corticosteroid-free response rates of patients on anti-TNFa monotherapy and combination therapy.

In the South Limburg area, anti-TNFo therapy is frequently being used in CD management [ $21.2 \%$ after one year and $41.2 \%$ after five years in the most-recent years] as compared to recent data from other large cohorts, such as a Danish national study [24\% after a mean follow-up of 7.7 years] and the ECCO-Epicom study [19\% within one year].24,25 Exposure rates were highest in patients with colonic or ileocolonic disease localization, patients with upper Gl or perianal disease, and smokers, all of which are associated with an unfavorable long-term disease outcome. ${ }^{1,2}$ In comparison to the baseline characteristics of the anti-TNF $\alpha$ registration trials populations, age, sex, and disease phenotype distributions were comparable, yet the median time to first anti-TNF $\alpha$ agent administration was considerably lower in our cohort [2.5 years vs. 7.7-9.1 years $\left.{ }^{4-7}\right]$, as well as in some ${ }^{13,14,26}$ but not all 12,15,22,27${ }^{29}$ other real-world cohorts. Differences in the timing of first anti-TNFa prescription between trials and real-life, but also among real-world studies, are important to acknowledge, as it is assumed that the effectiveness of anti-TNF $\alpha$ therapy is higher early in disease course.

The long-term effectiveness results from the present study showed that anti-TNF $\alpha$ agents have a durable response in a real-world setting. In our study, 50.1\% [IFX] and 60.9\% [ADA] of the anti-TNF $\alpha$ users still used the agent after five years and did not require corticosteroids, hospitalization or surgery. Insight in the long-term response of anti-TNF $\alpha$ agents through observational studies is important, because clinical trials only had a follow-up up to two years. Direct comparison of the response rates from our study with the ones from the registration trials is difficult, because of differences in response definition [i.e. our composite parameter of non-response vs. clinical activity scores] and study population [i.e. all patients starting anti-TNF $\alpha$ therapy vs. primary responders]. Other cohort studies used a definition of response that is more alike ours and reported rather similar 5-year IFX response rates [63.4-66.4\%]. ${ }^{14,15,27,28}$ Second, we found no association between the timing of initiating anti-TNF $\alpha$ therapy and the CSFR rate. However, firm conclusions on the timing of initiating therapy cannot be drawn from observational studies, since selection bias probably plays a major role [i.e. patients with severe disease, and thus poor prognosis, are most likely to receive anti-TNF $\alpha$ therapy early in disease course]. Third, we found an association between the CSFR 
rate and the calendar year of start of anti-TNFa therapy, with higher response rates in more recent years. In the multivariable analysis, this relation was found to be independent from temporal changes in patient or disease characteristics and also independent from the time between $\mathrm{CD}$ diagnosis and start of anti-TNF $\alpha$ therapy. This is an interesting finding, which may reflect a temporal change in treatment indication, optimization, and monitoring [e.g. therapeutic drug monitoring and serial measurements of fecal calprotectin], resulting in an improved durable response. Such effects are, however, difficult to assess in an observational study.

Combination therapy is considered to optimize the efficacy of anti-TNF $\alpha$ agents and inhibits the formation of antibodies, especially in IFX.30,31 For ADA, the beneficial effect of concurrent immunomodulator use seems less pronounced..$^{32}$ In the present study, we found no difference in achieving CSFR between CD patients on anti-TNF $\alpha$ monotherapy and combination therapy. Results were similar when only IFX patients were considered. Three large trials support the use of [early] combination therapy over anti-TNF $\alpha$ monotherapy with infliximab, by demonstrating higher corticosteroid-free remission rates ${ }^{33,34}$ or lower surgery rate on the long-term ${ }^{35}$ in the combination therapy arm. Some cohort studies also found better anti-TNF $\alpha$ response rates in combination with immunomodulators ${ }^{13,22,27,28,36}$, whereas other studies also did not find a significant relation. ${ }^{14,15}$ The discrepancy in current literature may be caused by local differences in the indication for combination therapy or by the employed methodological approach for addressing confounding. Referring to the latter, proper adjustment for confounding is needed, because the use of combination therapy and disease severity are strongly linked. Moreover, combination therapy requires a well-though-out definition, acknowledging situations in which the immunomodulator is being withdrawn or started during follow-up and acknowledging immune-time bias [i.e. unintentionally giving subjects a period of immunity for the event by the employed definition of treatment exposure]. ${ }^{37,38}$ In the present study, we attempted to minimize confounding by using propensity score matching to balance relevant characteristics between monotherapy and combination therapy users $^{39}$ and immune-time bias by censoring follow-up after immunomodulator discontinuation [in combination therapy users] or initiation [in monotherapy users]. Given the methodological complexity of studying the added value of combination therapy, other large cohorts with sufficient clinical detail should confirm our findings.

In clinical practice, IFX and ADA are the two most-used anti-TNF $\alpha$ agents. A head-tohead comparison trial has never been performed and therefore data on their comparative effectiveness must come from network analyses and observational studies, either studying large administrative databases or cohorts. In the present study, we found a similar effectiveness of IFX and ADA, also after adjusting for patient and disease characteristics via propensity score matching. In literature, data on the comparative effectiveness of IFX and ADA 
for CD are conflicting. Network analyses studies, which have assessed the comparative effectiveness via an indirect comparison of the clinical trials by using the placebo arm as 'comparator', have found the efficacy of IFX and ADA to be similar. 8,40 Two nationwide studies, using large claim-based databases from the United States, came to contradictory conclusions: Singh et al. found IFX to be superior to ADA in terms of a lower risk of hospitalization, surgery, and corticosteroid use, whereas Osterman et al. found no significant differences between agents. ${ }^{16,17}$ Another nationwide, claim-based study from Denmark also found no significant difference in effectiveness between IFX and ADA. ${ }^{41}$ Cohort studies also came to different conclusions. 12,13,22,26 The discrepancy in current literature may be the result of different methodological approaches to assess the comparative effectiveness of two drugs in an observational study design. For instance, it is relevant to acknowledge the non-random allocation of anti-TNFa agents over the patient population in an observational study. For example, IFX might be preferred above ADA in severe luminal disease in a hospitalized patient, as was observed in the present study. Second, sample size is important, as the expected differences in effectiveness between agents are small, given that both IFX and ADA are antiTNF $\alpha$ antibodies. Studies with a small sample size may miss potential [small] beneficial effects of one drug over another. Considering current literature, claim-based databases have a large sample size and hence can detect subtle differences between agents but lack clinical detail to adjust for confounding. Cohort studies have opposite characteristics. Ultimately, based on current literature, no firm conclusions can be drawn, yet it is likely that IFX and ADA exert a similar effect, although a small clinical benefit of IFX cannot be excluded.

The strength of the present study resides in the combination of a population-based design and a deep level of clinical detail. The former enhanced external validity by providing data from an unselected population of CD patients, while the latter enabled advanced adjustment for confounding. Study limitations include the sample size, which may have been too small to detect small differences in effectiveness, and the lack of information on drug safety [e.g. infections] which would have augmented the discussion on the long-term effectiveness of anti-TNFa therapy.

In conclusion, both IFX and ADA had durable response rates in a real-world cohort of unselected CD patients. Retreatment with anti-TNF $\alpha$ therapy after initial discontinuation is often successful. We found no superiority of one anti-TNFa agent over the other in clinical effectiveness. Therefore, the choice for an anti-TNF $\alpha$ agent can be based upon the preferred administration route and costs. 


\section{Supplement}

A

\section{Corticosteroid-free survival}

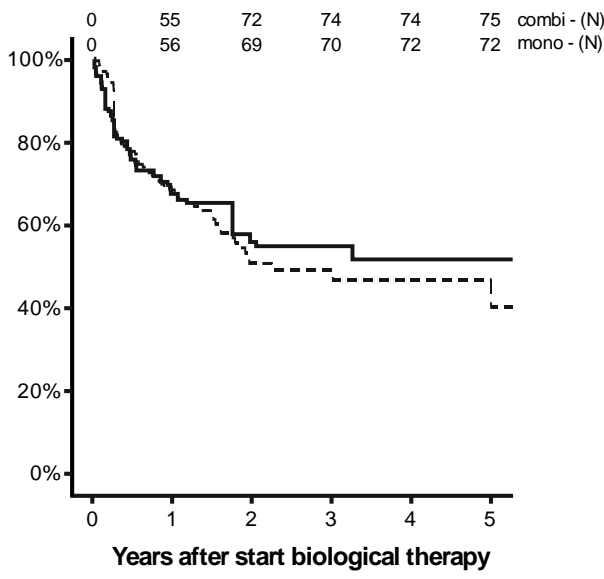

B

\section{Corticosteroid-free survival (IFX only)}

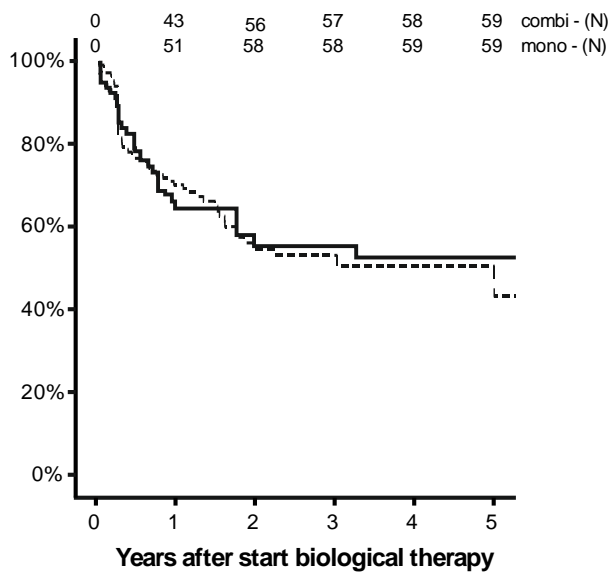

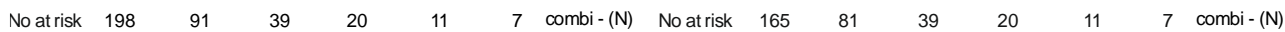

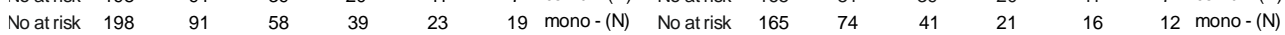

C

Risk of hospitalisation

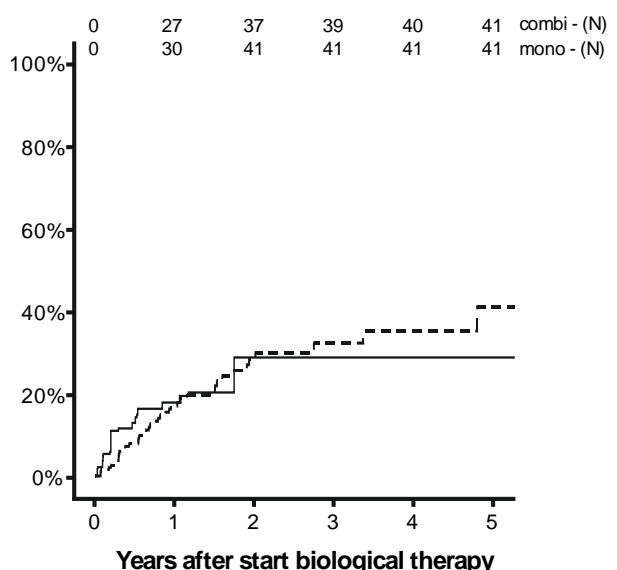

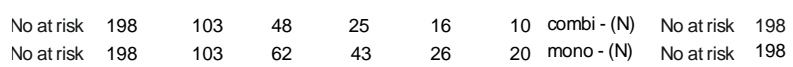

D

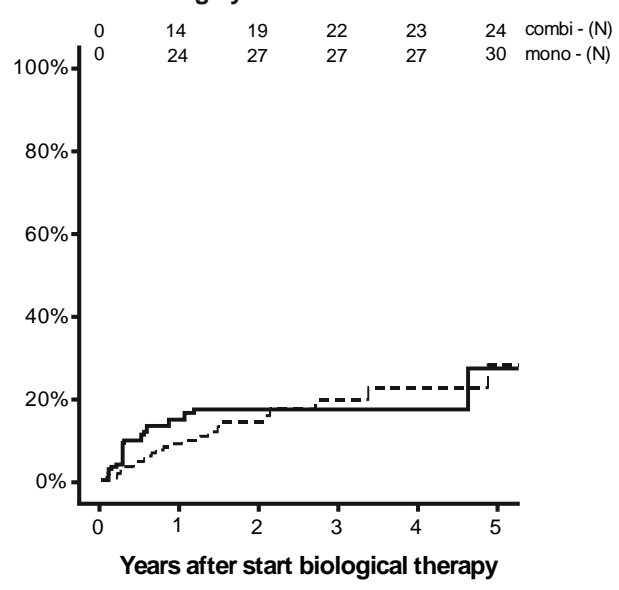

$\begin{array}{llllll}111 & 59 & 31 & 19 & 12 & \mathrm{combi}-(\mathrm{N})\end{array}$ monotherapy $\quad$ - - - combination therapy

Figure 9.S1. Long-term outcome of patients on anti-TNF $\alpha$ monotherapy and combination therapy in terms of cumulative corticosteroid-free response [A and B], hospitalization [C] and surgery [D].

IFX, infliximab. ADA, adalimumab. 


\section{References}

1. Solberg IC, Vatn $\mathrm{MH}$, Hoie $\mathrm{O}$, et al. Clinical course in Crohn's disease: results of a Norwegian population-based tenyear follow-up study. Clin Gastroenterol Hepatol. 2007;5(12):1430-8.

2. Jeuring SF, van den Heuvel TR, Liu LY, et al. Improvements in the Long-Term Outcome of Crohn's Disease Over the Past Two Decades and the Relation to Changes in Medical Management: Results from the Population-Based IBDSL Cohort. Am J Gastroenterol. 2017;112(2):325-36.

3. Peyrin-Biroulet L, Loftus EV, Jr., Colombel JF, et al. The natural history of adult Crohn's disease in populationbased cohorts. Am J Gastroenterol. 2010;105(2):289-97.

4. Hanauer SB, Feagan BG, Lichtenstein $G R$, et al. Maintenance infliximab for Crohn's disease: the ACCENT I randomised trial. Lancet. 2002;359(9317):1541-9.

5. Hanauer SB, Sandborn WJ, Rutgeerts P, et al. Human anti-tumor necrosis factor monoclonal antibody (adalimumab) in Crohn's disease: the CLASSIC-I trial. Gastroenterology. 2006;130(2):32333; quiz 591.

6. Sandborn WJ, Hanauer SB, Rutgeerts P, et al. Adalimumab for maintenance treatment of Crohn's disease: results of the CLASSIC II trial. Gut. 2007;56(9):1232-9.

7. Colombel JF, Sandborn WJ, Rutgeerts P, et al. Adalimumab for maintenance of clinical response and remission in patients with Crohn's disease: the CHARM trial. Gastroenterology. 2007;132(1):52-65.

8. Hazlewood GS, Rezaie A, Borman M, et al. Comparative effectiveness of immunosuppressants and biologics for inducing and maintaining remission in
Crohn's disease: a network metaanalysis. Gastroenterology. 2015;148(2):344-54 e5; quiz e 14-5.

9. Concato J, Shah N, Horwitz RI. Randomized, controlled trials, observational studies, and the hierarchy of research designs. N Eng/ J Med. 2000;342(25):1887-92.

10. Salleron J, Danese S, D'Agay L, et al. Effectiveness Research in Inflammatory Bowel Disease: A Necessity and a Methodological Challenge. J Crohns Colitis. 2016;10(9):1096-102.

11. Ha C, Ullman TA, Siegel CA, et al. Patients enrolled in randomized controlled trials do not represent the inflammatory bowel disease patient population. Clin Gastroenterol Hepatol. 2012;10(9):1002-7; quiz e78.

12. Patil $S A$, Rustgi $A$, Langenberg $P$, et al. Comparative effectiveness of anti-TNF agents for Crohn's disease in a tertiary referral IBD practice. Dig Dis Sci. 2013;58(1):209-15.

13. Ananthakrishnan AN, Cagan A, Cai T, et al. Comparative Effectiveness of Infliximab and Adalimumab in Crohn's Disease and Ulcerative Colitis. Inflamm Bowel Dis. 2016;22(4):880-5.

14. Billiet $T$, Cleynen I, Ballet $V$, et al. Prognostic factors for long-term infliximab treatment in Crohn's disease patients: a 20-year single centre experience. Aliment Pharmacol Ther. 2016;44(7):673-83.

15. Schnitzler $F$, Fidder $H$, Ferrante $M$, et al. Long-term outcome of treatment with infliximab in 614 patients with Crohn's disease: results from a single-centre cohort. Gut. 2009;58(4):492-500. Epub 2008/10/04.

16. Osterman MT, Haynes K, Delzell E, et al. Comparative effectiveness of infliximab and adalimumab for Crohn's disease. 
Clin Gastroenterol Hepatol.

2014;12(5):811-7 e3.

17. Singh S, Heien HC, Sangaralingham LR, et al. Comparative Effectiveness and Safety of Anti-Tumor Necrosis Factor Agents in Biologic-Naive Patients With Crohn's Disease. Clin Gastroenterol Hepatol. 2016;14(8):1120-9 e6.

18. van den Heuvel TR, Jonkers DM, Jeuring SF, et al. Cohort Profile: The Inflammatory Bowel Disease South Limburg Cohort (IBDSL). Int J Epidemiol. 2017;46(2):e7.

19. Satsangi J, Silverberg MS, Vermeire S, et al. The Montreal classification of inflammatory bowel disease: controversies, consensus, and implications. Gut. 2006;55(6):749-53.

20. Dignass A, Van Assche G, Lindsay JO, et al. The second European evidence-based Consensus on the diagnosis and management of Crohn's disease: Current management. J Crohns Colitis. 2010;4(1):28-62.

21. van Bodegraven $A A$, van Everdingen $\mathrm{JJ}$, Dijkstra G, et al. [Guideline 'Diagnosis and treatment of inflammatory bowel disease in adults'. I. Diagnosis and treatment]. Nederlands tijdschrift voor geneeskunde. 2010;154:A1899. Epub 2010/10/30. Richtlijn 'Diagnostiek en behandeling van inflammatoire darmziekten bij volwassenen'. I. Diagnostiek en behandeling.

22. Kestens $C$, van Oijen MG, Mulder $C L$, et al. Adalimumab and infliximab are equally effective for Crohn's disease in patients not previously treated with antitumor necrosis factor-alpha agents. Clin Gastroenterol Hepatol. 2013; 11(7): 826-31.

23. Van Assche G, Dignass A, Panes J, et al. The second European evidence-based Consensus on the diagnosis and management of Crohn's disease: Definitions and diagnosis. J Crohns Colitis. 2010;4(1):7-27.
24. Vester-Andersen MK, Vind I, Prosberg $\mathrm{MV}$, et al. Hospitalisation, surgical and medical recurrence rates in inflammatory bowel disease 20032011-a Danish population-based cohort study. J Crohns Colitis. 2014;8(12):1675-83.

25. Vegh Z, Burisch J, Pedersen N, et al. Treatment Steps, Surgery, and Hospitalization Rates During the First Year of Follow-up in Patients with Inflammatory Bowel Diseases from the 2011 ECCO-Epicom Inception Cohort. J Crohns Colitis. 2015;9(9):747-53.

26. Tursi A, Elisei W, Picchio $M$, et al. Effectiveness and safety of infliximab and adalimumab for ambulatory Crohn's disease patients in primary gastroenterology centres. Eur. J. Intern. Med. 2014;25(5):485-90.

27. Rudolph SJ, Weinberg DI, McCabe RP. Long-term durability of Crohn's disease treatment with infliximab. Dig Dis Sci. 2008;53(4):1033-41

28. Chaparro M, Panes J, Garcia V, et al. Long-term durability of infliximab treatment in Crohn's disease and efficacy of dose "escalation" in patients losing response. J Clin Gastroenterol. 2011;45(2):113-8.

29. Panaccione R, Colombel JF, Sandborn WJ, et al. Adalimumab sustains clinical remission and overall clinical benefit after 2 years of therapy for Crohn's disease. Aliment Pharmacol Ther. 2010;31(12):1296-309.

30. Hanauer $\mathrm{SB}$, Wagner $\mathrm{CL}$, Bala M, et al. Incidence and importance of antibody responses to infliximab after maintenance or episodic treatment in Crohn's disease. Clin Gastroenterol Hepatol. 2004;2(7):542-53.

31. Baert F, Noman M, Vermeire S, et al. Influence of immunogenicity on the longterm efficacy of infliximab in Crohn's disease. N Eng/ J Med. 2003;348(7): 601-8 
32. Chalhoub JM, Rimmani HH, Gumaste W, et al. Systematic Review and Metaanalysis: Adalimumab Monotherapy Versus Combination Therapy with Immunomodulators for Induction and Maintenance of Remission and Response in Patients with Crohn's Disease. Inflamm Bowel Dis. 2017;23(8):131627.

33. Colombel JF, Sandborn WJ, Reinisch W, et al. Infliximab, azathioprine, or combination therapy for Crohn's disease. N Eng/ J Med. 2010;362(15):1383-95.

34. D'Haens G, Baert F, van Assche G, et al. Early combined immunosuppression or conventional management in patients with newly diagnosed Crohn's disease: an open randomised trial. Lancet. 2008;371(9613):660-7

35. Khanna R, Bressler B, Levesque BG, et al. Early combined immunosuppression for the management of Crohn's disease (REACT): a cluster randomised controlled trial. Lancet. 2015;386(10006):1825-34.

36. Sokol H, Seksik P, Carrat F, et al. Usefulness of co-treatment with immunomodulators in patients with inflammatory bowel disease treated with scheduled infliximab maintenance therapy. Gut. 2010;59(10):1363-8.

37. Suissa S. Immortal time bias in observational studies of drug effects. Pharmacoepidemiol Drug Saf. 2007;16(3):241-9.

38. Targownik LE, Suissa S. Understanding and Avoiding Immortal-Time Bias in Gastrointestinal Observational Research. Am J Gastroenterol. 2015;110(12): 1647-50

39. Austin PC. An Introduction to Propensity Score Methods for Reducing the Effects of Confounding in Observational Studies. Multivariate Behav Res. 2011;46(3): 399-424

40. Stidham RW, Lee TC, Higgins PD, et al. Systematic review with network meta- analysis: the efficacy of anti-TNF agents for the treatment of Crohn's disease.

Aliment Pharmacol Ther. 2014;39(12):1349-62.

41. Singh S, Andersen NN, Andersson M, et al. Comparison of infliximab with adalimumab in 827 biologic-naive patients with Crohn's disease: a population-based Danish cohort study. Aliment Pharmacol Ther. 2018;47(5):596-604. 



\section{CHAPTER 10}

General discussion

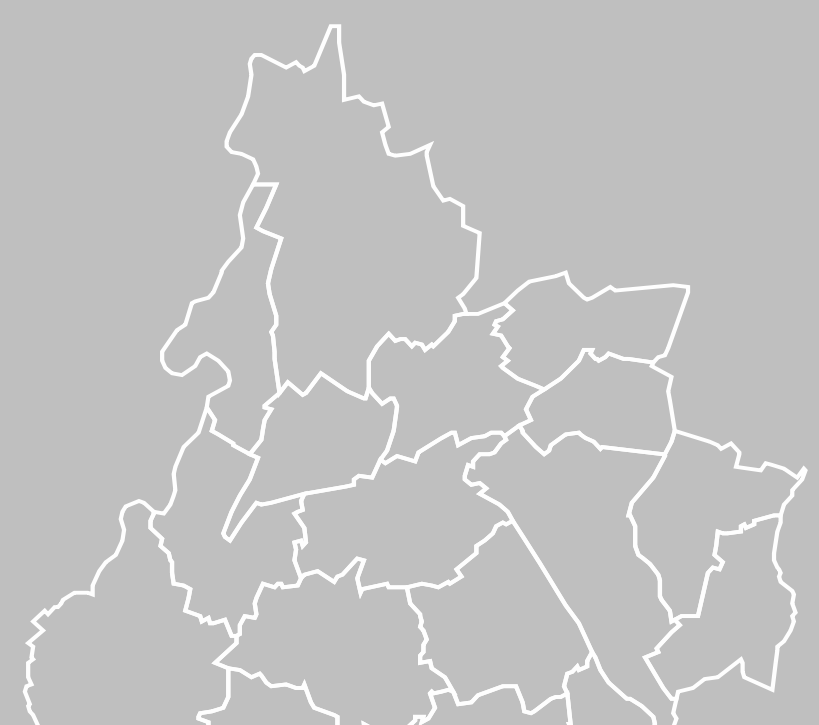




\section{General discussion}

In this thesis, temporal changes in the epidemiology of inflammatory bowel disease [IBD] were studied in the population of South Limburg, the Netherlands, between 1991 and 2014. Changes in disease incidence and outcome were assessed, with special focus on the question whether advances in IBD management over the past two decades have resulted in an improved disease outcome.

In brief, demographic, clinical and medication data were collected from patients diagnosed with IBD in the area of South Limburg as part of the IBD South Limburg [IBDSL] cohort and formed the basis for all studies. Over time, the incidences of both Crohn's disease [CD] and ulcerative colitis [UC] increased markedly. The long-term outcome of IBD has improved in terms of cumulative corticosteroid use and in the risks of requiring hospitalization [CD only] and surgery. However, no change was found in the risk of developing intestinal strictures and/or fistulas, despite pronounced changes in the medical management of IBD.

In this chapter, the conclusions from all studies are put into perspective and the implications for the future of IBD management will be discussed.

\section{Challenges in epidemiological research on IBD}

Pharmacoepidemiological studies in the field of IBD aid to provide insight in the burden of disease on the health care system and society, in the real-world effectiveness of current treatment modalities and in disease outcomes of specific patient subgroups, particularly those who are often excluded from clinical trials, such as the elderly. The validity of such studies largely depends on the degree to which bias and confounding can be minimized, warranting a careful selection of the study population and the statistical approaches.

When studying the incidence and long-term outcome of IBD, it is important to identify all IBD patients in the population. Suboptimal identification and ascertainment of cases can result in selection bias and incorrect estimates of the disease burden. Data on IBD epidemiology mainly derive from hospital-based cohorts, population-based cohorts or claimbased registries. An advantage of the latter is the high number of included patients, which results in high statistical power. However, case ascertainment is often confined, and, consequently, such studies may suffer from misclassification and may overrepresent patients who frequently employ healthcare resources. ${ }^{1-2}$ Additionally, clinical data often lack detail, impeding the statistical possibilities for addressing confounding. Cohort studies have the advantage of direct patient identification and a deeper level of detail of the clinical data. Yet, the number of patients is often lower, which can be a problem if infrequently observed outcome measures are being studied. Population-based cohort studies have the advantage of high external validity, as they reflect the full spectrum of disease, including milder cases. These 
may be missed in hospital-based cohorts if patients do not or rarely attend the [referral] hospital. Recently, Van den Heuvel et al. showed that hospital-based cohorts indeed have an overrepresentation of IBD patients with characteristics of severe disease as compared to population-based cohorts. ${ }^{3}$ In Chapter 2, we described the design of the population-based IBDSL cohort and the efforts taken to capture the majority of incident cases in South Limburg. Patients diagnosed with IBD as from 1991 were identified via a multi-faceted approach and completeness was cross-checked retrospectively with data from the national pathology database PALGA and regional general practitioners. Furthermore, IBD diagnosis was confirmed for each patient individually, via manual review of medical records. Supported by a stable source population and a close cooperation between hospitals and general practitioners in the region, 93\% of the IBD population in South Limburg was included in the IBDSL registry. Although there is no clear threshold value for 'cohort completeness' to consider a cohort study truly population-based, we consider our completeness high enough to designate IBDSL as population-based cohort and its results to reflect the impact IBD has on the local health care system and society.

A second challenge in epidemiological research is to limit bias and confounding. By employing regression statistics, the relation between medical therapy and the long-term disease outcome can be studied to reflect on the clinically relevant question whether the [early] use of a specific drug can alter the natural history of IBD. However, in such analyses, immune time bias needs to be considered, as this can lead to a profound overestimation of the effects of medical therapy, if inadequately addressed in the study design. Immune time bias occurs when the time between disease diagnosis and the start of medical therapy [time in which a patient is not exposed to a specific drug] is either misattributed as exposure time or ignored. ${ }^{4.5}$ Patients on a specific treatment are then credited with time in which the event of interest could not have taken place by means of the definitions used in the study. In 2015, Targownik et al. stated that conclusions from many studies on the association between immunomodulator use and surgery risk [some of which found a surgery risk reduction up to $\left.71 \%{ }^{6-13}\right]$ were 'too good to be true' and had to be ascribed to immune time bias. ${ }^{14}$ In Chapters 5 and 8 , we studied the relation between medication use and the long-term outcome of CD and corticosteroid use, respectively, and endeavored to minimize the effect of this form of bias by using propensity score modeling and subsequent matching of the follow-up between users and nonusers. This 'conservative' statistical approach improves the validity of our results and may explain the discrepancy in conclusion between our study and previous reports.

In conclusion, obtaining valid and generalizable epidemiological data is challenging. It is important to minimize bias and confounding already in the design of the cohort. Large population-based cohorts are preferred as they study the total population, have high case ascertainment and represent the full spectrum of disease. Nevertheless, all study types come 
with advantages and disadvantages, which need to be acknowledged in the interpretation of epidemiological literature on IBD.

\section{Implications of the rising incidence of IBD}

In a systematic review, Molodecky et al. demonstrated that the incidence of IBD has increased throughout the world over the past decades. ${ }^{15}$ Moreover, they showed that the incidence and prevalence, including their temporal trends, vary widely between continents, countries and even regions. Therefore, extrapolation of epidemiological data to other regions may result in a distorted view on the local disease burden, which underlines the need for local data. At the start of this thesis, no recent data for the Netherlands were available.

In Chapter 3, we reported that the incidence rates of CD and UC in South Limburg showed annual increases of $6 \%$ and $4 \%$, respectively. Insight in the potential causes of these increases may identify targets for intervening measures. First, it is important to examine whether the observed increases point to 'true' incidence increases or that they must be ascribed to methodological factors in study design or to changes in the diagnostic process [i.e. former underdiagnosing]. Methodological factors, however, are not likely to explain the rising incidence in South Limburg, given the stable in- and exclusion criteria of the IBDSL cohort over time and the thorough patient identification and ascertainment. Referring to changes in the diagnostic approach, it should be noted that ileocolonoscopies are currently more often performed than sigmoidoscopies as compared to the early nineties, and small bowel imaging via computed tomography $[\mathrm{CT}]$ and magnetic resonance imaging [MRI] is also more common. Furthermore, the introduction of fecal calprotectin as screening instrument for intestinal inflammation may have played a role. ${ }^{16}$ However, it seems unlikely that underdiagnosis fully explains the increasing incidence, considering the symptoms, chronicity and severity of IBD. Moreover, the ongoing incidence increase in more recent years also points towards other factors than underdiagnosis. If the rising incidence does reflect a true increase, environmental factors likely play a role. Supportive of the former is the short period of time in which the incidence increase occurred, which is too short for genetic factors to exert an effect. Meta analyses reported on associations between IBD and factors related to a 'Western lifestyle', including diet ${ }^{17}$, smoking ${ }^{18}$, urbanization ${ }^{19}$ and air pollution ${ }^{20-22}$. Therefore, the results presented in Chapter 3 served as a starting point for an environmental risk factor analysis in the South Limburg area. A recent geographic information study [GIS] by Van den Heuvel et al. found, however, no significant association between ambient air quality and the onset of IBD in South Limburg. ${ }^{23}$ Studying environmental factors is challenging, given the variation in exposure during day and life [e.g. traveling from home to work, moving to another region], complex relations between host and environment [ e.g. host susceptibility, co-exposure of other environmental factors], and varying time between exposure and the moment its effect is 
notable. The complex interaction between environment and host during life is commonly referred to as the 'exposome' and is an upcoming field in epidemiological research. ${ }^{24-25}$ In future, scientists may find leads for unraveling the complex etiology of IBD by studying the totality of exposures as from gestation onwards, although such work is unprecedented in complexity.

Meanwhile, the incidence of IBD is increasing. Acknowledging the young age at disease onset and the unaffected low mortality rate, the IBD prevalence rate increases accordingly. Based on an extrapolation of the incidence in South Limburg, it was estimated that the nationwide prevalence was 80,627 patients in 2010 and a further extrapolation to 2019 indicates that the current prevalence exceeds 90,000 patients [data not shown]. Such numbers illustrate that IBD puts increasing pressure on society in general and on the health care system in particular. For example, IBD patients have a high risk of work disability: 18.3\% of the CD and $9.5 \%$ of the UC patients were fully disabled from work in a Dutch multicenter study. ${ }^{26}$ Physical problems such as fatigue are also reported, even in periods of disease remission. ${ }^{27}$ Along with emotional and social problems, this will further impair quality of life. ${ }^{28}$ Regarding the health care system, it is challenging to maintain good quality of care with current resources if the disease prevalence rises quickly. The concomitant changes in treatment goals towards a tighter monitoring of mucosal inflammation put further pressure on the system. Therefore, it is crucial to find new, efficient strategies for managing and following IBD patients in order to maintain high-quality care, yet to avoid a subsequent profound increase in health care costs. Disease management with telemedicine tools is gaining interest, supported by the increasing use of internet devices and the widespread ability to utilize such devices in the general population. A large Dutch randomized controlled trial with the telemonitoring tool MijnIBDCoach ${ }^{29}$ showed that the group of IBD patients using remote monitoring required less outpatient visits and hospitalizations than the group of patients who received standard care, while safety and patient satisfaction were maintained. Via e-learnings, monthly questionnaires and an user-friendly and accessible communication module to contact specialized IBD nurses, patient empowerment is also supported. ${ }^{29}$ Previously, Robinson et al. suggested that enhancing patient empowerment may lead to a better treatment response, better patient satisfaction and more efficient health resource utilization. ${ }^{30}$ Future studies must confirm the beneficial effects of telemonitoring systems in IBD and its cost-effectiveness for managing the rising IBD patient population. 


\section{Two decades of advances in IBD management: new era, better future?}

As discussed in Chapter 1, IBD can be invalidating due to symptoms, frequent hospitalizations and [multiple] surgical resections, but also because of work disability and an impaired quality of life. In IBD epidemiology, classic endpoints are hospitalization [as proxy for severe disease requiring either medical or surgical in-hospital treatment] and surgery. Surgery is one of the events patients fear most ${ }^{28}$, despite its well-established role in the treatment of IBD with good long-term results, also in terms of quality of life. ${ }^{31}$ Although merely studied due to the complexity of data collection, the cumulative exposure to corticosteroids during disease course is also a clinically relevant outcome parameter, as corticosteroid sparing is an important treatment goal in international guidelines. ${ }^{32}$ Retrieving insight in the evolution of the aforementioned outcome measures over time is not only important to justify the increasing use of immune modulating medication ${ }^{33-35}$ and high health care costs of $\mathrm{IBD}^{36-37}$, but also to find targets for further optimization of IBD care.

In this thesis, we conclude that the long-term disease outcomes of CD and UC in South Limburg has improved in terms of surgery risk, hospitalization risk [CD only] and in the cumulative use of corticosteroids. An overview of the temporal trends in these endpoints in population-based IBD literature, including ours, is presented in Table 10.1. In summary, many population-based studies found decreasing surgery rates, with notable similarities between studies, illustrating the external validity of population-based cohorts. Although various studies described trends in disease outcome, only a few elaborated on causes. In Chapters 5 and 6 , we proposed that changes in the diagnostic process [e.g. earlier disease diagnosis, different management of the first flare] played an important role in the long-term avoided hospitalizations and surgeries. Supportive of the former is the observation that the hospitalization [CD] and surgery [CD and UC] rates already decreased at, or shortly after, diagnosis, while only a limited number of patients had been exposed to immunomodulator or anti-TNF $\alpha$ therapy at that time. In CD, however, also hospitalizations and surgeries later in disease course were found to be avoided, which indicates that changes in medical management may have played a role. The expectations of medical therapy in changing longterm outcome measures are high, driven by the paradigm shift towards an earlier and more frequent use of immunomodulators and, particularly, anti-TNF $\alpha$ therapy. In the IBDSL cohort, this shift was indeed observed: the number of patients on immunomodulator therapy within the first year of disease quintupled over time to approximately 10\% in UC and 50\% in CD in era 2006-2011. For anti-TNF $\alpha$ therapy, the 1-year exposure rates increased from $0 \%$ to approximately $4 \%$ and $21 \%$, respectively. 
Table 10.1. Disease outcome parameters in the IBDSL cohort and other population-based cohorts

\begin{tabular}{|c|c|c|c|c|c|c|c|c|}
\hline & Crohn's & disease & & & Ulcerat & e colitis & & \\
\hline \multicolumn{9}{|c|}{ IBDSL, the Netherlands } \\
\hline & '91-’98 & '99-’05 & '06-'11 & & '91-'97 & '98-'05 & '06-'10 & \\
\hline $\begin{array}{l}\text { Cumulative } 5 \text {-year } \\
\text { hospitalization rate }\end{array}$ & $65.9 \%$ & $53.1 \%$ & $44.2 \%$ & $p<0.01$ & $22.3 \%$ & $19.5 \%$ & $18.3 \%$ & $p=0.08$ \\
\hline $\begin{array}{l}\text { Cumulative 5-year } \\
\text { surgery rate }\end{array}$ & $42.9 \%$ & $26.2 \%$ & $17.4 \%$ & $p<0.01$ & $7.5 \%$ & $5.7 \%$ & $4.1 \%$ & $p<0.01$ \\
\hline $\begin{array}{l}\text { Cumulative 5-year } \\
\text { phenotype } \\
\text { progression rate }\end{array}$ & $21.2 \%$ & $21.7 \%$ & $21.3 \%$ & $p<0.01$ & - & - & - & - \\
\hline \multicolumn{9}{|c|}{ Nationwide, Denmark ${ }^{33}$} \\
\hline & '87-'94 & '95-’02 & '03-'11 & & '87-'94 & '95-'02 & '03-'11 & \\
\hline $\begin{array}{l}\text { Cumulative 5-year } \\
\text { surgery rate }\end{array}$ & $41.7 \%$ & $26.7 \%$ & $19.6 \%$ & $p<0.01$ & $11.8 \%$ & $8.4 \%$ & $7.5 \%$ & $p<0.01$ \\
\hline
\end{tabular}

\section{Cardiff, United Kingdom ${ }^{34}$}

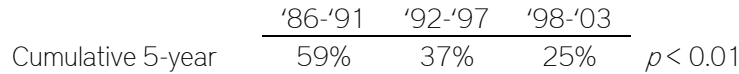

\section{surgery rate}

\section{UMIBD, Canada ${ }^{38}$}

Cumulative 5-year

\begin{tabular}{|c|c|c|}
\hline '92-'96 & '97-'01 & '02-'08 \\
\hline $8.7 \%$ & $7.0 \%$ & $5.8 \%$ \\
\hline
\end{tabular}

surgery rate

\section{OImsted County, USA ${ }^{39}$}

Cumulative 5-year hospitalization rate

Cumulative 5-year

\begin{tabular}{cc}
$' 90-{ }^{\prime} 99$ & $' 00-{ }^{\prime} 04$ \\
\hline $28.6 \%$ & $44.2 \%$ \\
$13.1 \%$ & $24.1 \%$
\end{tabular}
$p=0.14$

surgery rate

\begin{tabular}{|c|c|c|c|c|}
\hline \multicolumn{5}{|c|}{ Veszprem, Hungary $^{42}$} \\
\hline & '77-'89 & ‘90-'98 & '99-'08 & \\
\hline $\begin{array}{l}\text { Cumulative } 5 \text {-year } \\
\text { surgery rate }\end{array}$ & - & $35 \% *$ & $25 \%$ & $p=0.02$ \\
\hline $\begin{array}{l}\text { Cumulative 5-year } \\
\text { phenotype } \\
\text { progression rate }\end{array}$ & $35.5 \%$ & $25.5 \%$ & $15.2 \%$ & $p=0.02$ \\
\hline
\end{tabular}

$\mathrm{IQR}$, interquartile range

* patients from cohort 1977-1989 and cohort 1990-1998 were merged for this analysis 
However, as previously discussed, assessing the relation between temporal changes in medical management and temporal changes in disease outcome is complex and misinterpretation of data due to bias and confounding looms. By using an adequate statistical approach, including proper assignment of follow-up time to avoid immune-time bias, and by employing a propensity score matching technique, we assessed the relation between medication use and the long-term disease outcome. In contrast to some other observational studies, we found no statistically significant association between immunomodulator or antiTNF $\alpha$ use and the risk of hospitalization or surgery. Though, our results should also be interpreted with care, as statistical power was hampered by our more 'conservative' statistical approach. On the contrary, by using a similar approach, we found a statistically significant inverse association between immunomodulator and anti-TNF $\alpha$ use and the need for a course of corticosteroids, illustrating that [early] use of the corticosteroid sparing agents has indeed resulted in a diminished use of corticosteroids at population level. Other temporal changes that may have affected the change in disease outcome include advancements in IBD care [e.g. IBD nurses, multidisciplinary consultation, regular visits with availability of biochemical markers and patient education and empowerment] and changes in healthcare in general [e.g. striving for outpatient rather than in-hospital care, limiting the duration of hospital admission]. The effects of such factors are, however, difficult to quantify in an observational study design.

In contrast to the declining hospitalization and surgery rates, and the attenuated cumulative corticosteroid use, we observed no improvement in the risk of developing diseaserelated complications in CD. Complicated disease [i.e. the presence of intestinal fistulas/abscesses or strictures] and perianal fistulas are thought to be a consequence of longstanding insufficiently controlled mucosal inflammation ${ }^{40-41}$ and are associated with high risks of hospitalization and surgery, and with an impaired quality of life. ${ }^{6,33,34,42}$ This outcome parameter is of increasing importance, as prevention of structural bowel damage is currently suggested as a novel therapeutic goal in CD management, being driven by high expectations of biological therapy and new treatment strategies. ${ }^{43,44}$ Based on our data, current treatment options fail to prevent structural bowel damage on the long-term in real-life, at least at population level under the present treatment strategy. Whether the failure is indeed caused by insufficiently controlled mucosal inflammation remains to be elucidated. Nevertheless, our observation underlines the need for new therapeutic approaches in order to improve the outcome of IBD. 


\section{The future of IBD management: how to turn the tide?}

In literature, various strategies are proposed to improve the long-term disease outcome of $\mathrm{IBD}$.

Novel IBD medication A hallmark in the medical management of IBD was the introduction of biological agents in the late nineties of the previous century. Anti-TNFa therapy was the first biological therapy for IBD and its introduction took place in the running time of our IBDSL cohort. As the infliximab [IFX] and adalimumab [ADA] registration trials only had a limited follow-up of 52-56 weeks and included a diverse IBD population [e.g. both surgery-naive and post-surgical disease and both uncomplicated and complicated disease], it is impossible to draw conclusions on the effect of anti-TNF $\alpha$ therapy on the long-term outcome of IBD from these studies. ${ }^{45-51}$ Based on our previously discussed findings, the availability of anti-TNF $\alpha$ therapy has not changed the risks of hospitalization or surgery, but played a role in the attenuated use of corticosteroids. After completion of the data collection for the studies presented in this thesis, vedolizumab [anti-integrin $\alpha 4 \beta 7$ ], ustekinumab [interleukin 12/23 antagonist] and tofacitinib [Janus kinase inhibitor] were registered for IBD. Whether these agents have the potential to change the long-term outcome of IBD remains to be elucidated. Large real-world population-based cohorts such as the IBDSL cohort may prove useful in these. $^{52}$

Novel treatment strategy Novel treatment strategies include the 'accelerated step-up' and 'top-down' approach [see also Chapter 1]. The rationale of an early aggressive therapeutic approach comes from the field of rheumatology, in which beneficial effects on long-term joint damage were observed if disease-modifying anti-rheumatic drugs were given early in disease course. ${ }^{53-61}$ In IBD, two trials failed to show a beneficial effect of early immunomodulator use on the long-term corticosteroid-free remission rate [the AZTEC ${ }^{62}$ and RAPID trial ${ }^{63}$ ]. However, both had some methodological limitations, such as the lack of measuring drug trough levels and thiopurine methyltransferase status prior to therapy initiation, which may have led to more frequent treatment failure or discontinuation. Support for an early use of immunomodulators comes from retrospective cohort studies, which found lower surgery rates in patients who started immunomodulator therapy shortly after diagnosis. ${ }^{6-13}$ However, as previously discussed, most of these studies are subject to immune time bias, and, consequently, the surgery-sparing effects were probably overestimated. In the IBDSL cohort, we indeed observed that both immunomodulators and anti-TNF $\alpha$ agents were prescribed at an earlier stage in disease course in more recent years [Chapters 5 and 6], but we only found an association with a lower need of corticosteroids.

The top-down approach is a form of early intervention strategy, in which combination therapy [i.e. a combination of an anti-TNF $\alpha$ agent and an immunomodulator] is the initial 
therapy and treatment is de-escalated based on clinical, biochemical and/or endoscopic findings. The beneficial effects of combination therapy consisting of IFX and an immunomodulator are proven [see below], whereas the added value of adding an immunomodulator to ADA therapy is less clear. ${ }^{64}$ Four large clinical trials assessed the effects of early combined immunosuppressive therapy: the TOP-DOWN trial ${ }^{65}$ [inclusion from 2001 2004], the SONIC trial ${ }^{66}$ [inclusion from 2005-2008], the UC SUCCESS trial ${ }^{67}$ [inclusion from 2007-2010] and the REACT trial ${ }^{68}$ [inclusion from 2010-2013]. Superiority of combination therapy over IFX or azathioprine monotherapy in achieving corticosteroid-free remission was proven in the TOP-DOWN and SONIC trials for CD and in the UC SUCCESS trial for UC. However, complexities in the employed study designs limit the external validity and, hence, the implications for clinical practice. For instance, allowance of treatment escalation in the control arm of the TOP-DOWN study resulted in a rather similar anti-TNF $\alpha$ exposure in the early intervention and conventional treatment arm rate at week 52 [16\% vs 8\%] and 104 [8\% vs. $21 \%]$. However, treatment escalation in the control arm is inevitable, because one has to avoid unethical undertreatment of patients in such trials. Other methodological factors that hamper the interpretation of the results include the use of a cross-sectional rather than a longitudinal endpoint [e.g. risk of treatment failure at week 52 as compared to number of days/trimesters spent in remission] and the inclusion of patients with longstanding disease. Well-designed randomized clinical trials [RCTs] in therapy-naive patients with a long follow-up of at least two years and longitudinal endpoints can fill this gap of knowledge, but such trials are unlikely to be initiated due to the methodological complexity and limited resources. Furthermore, specific subgroups, such as the elderly and patients with moderate to severe comorbidities, are unlikely to be included in such trials. Therefore, real-world data, preferably systematically recorded, remain crucial to study clinically relevant treatment questions.

Tight disease management The goal of tight disease management ['tight control'] is to keep mucosal inflammation at a low level or, preferably, absent, so that the risk of aggravation to refractory, inflammatory disease, and subsequent structural organ damage is reduced [Figure 10.1]. In rheumatology, studies have shown that patients with rheumatoid arthritis [RA] who were more strictly monitored [e.g. planning regular visits, use of objective disease activity scores, early step-up of medical therapy] not only had a higher treatment response and were more likely to be in disease remission on the short-term, but also had less severe joint damage on the long-term. . $^{6-70}$ 


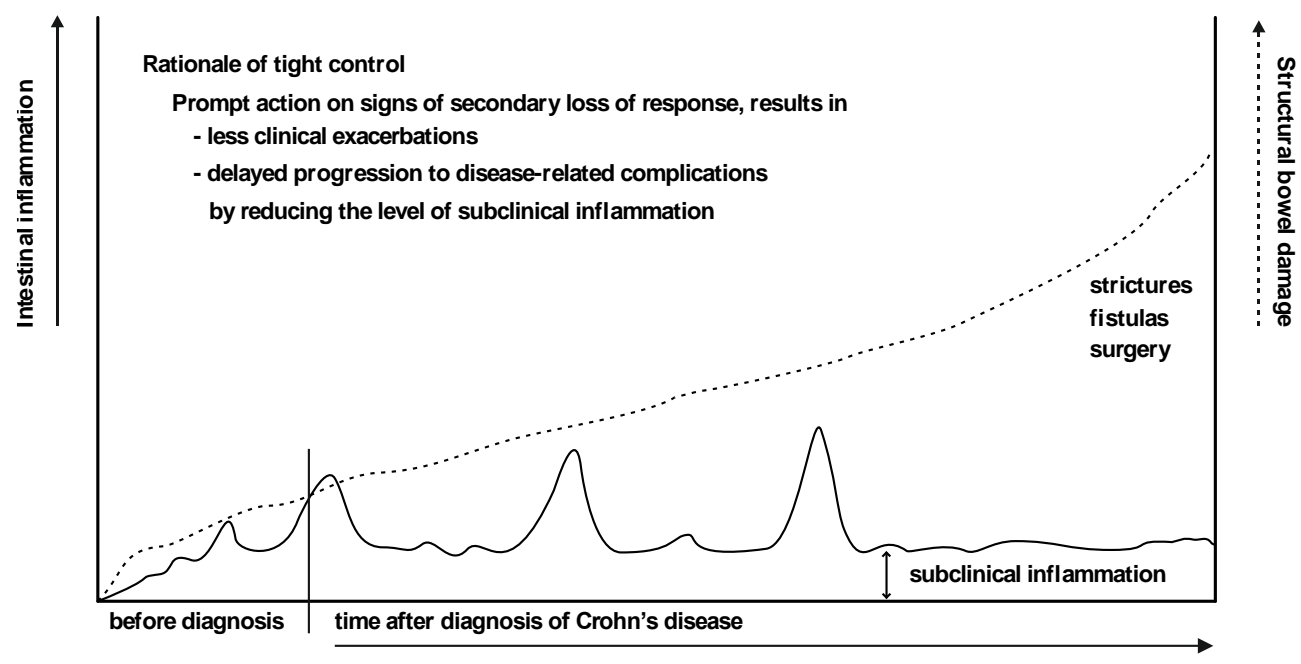

Figure 10.1. Rationale of the tight control treatment strategy. Intestinal inflammation is illustrated by the solid line. Structural bowel damage is illustrated by the dashed line.

Tight control in IBD encompasses regular checkups of clinical activity scores, blood [e.g. inflammatory markers] and fecal [e.g. calprotectin] markers. In particular the calprotectin assay enables non-invasive disease monitoring. Studies showed that serial calprotectin measurements can predict clinical relapse with sensitivity and specificity values ranging from 50-90\%. ${ }^{71-73}$ Moreover, calprotectin was also found to correlate well with endoscopic inflammation. ${ }^{74-75}$ First evidence for beneficial outcomes of tight control in IBD comes from the recent CALM trial, which showed that the use of biochemical markers in addition to symptoms resulted in higher rates of endoscopic remission [45.9\% in the tight control arm vs. $30.3 \%$ in the conventional therapy arm, $p=0.01]$ and corticosteroid-free remission [59.8\% vs. $39.3 \%, p<0.01$ ] at week $48 . .^{76}$ In our studies, laboratory data on the use of calprotectin were not available for the total cohort.

Therapeutic drug monitoring [TDM] of immunomodulators and biologicals is also part of tight control management of IBD. TDM encompasses the measurement of drug trough levels and the presence of antidrug antibodies if loss of response is suspected [reactive TDM] or at regular intervals [proactive TDM]. In CD, studies on reactive TDM have shown that patients who have adequate serum levels of IFX or ADA at the time of secondary loss of response benefit more from a switch out-of-class rather than from dose escalation or switch to another anti-TNF $\alpha$ agent. ${ }^{77-78}$ In contrast, two recent trials failed to show benefit of proactive trough level guided dosing of IFX over symptoms and/or biomarkers on corticosteroid-free remission. ${ }^{79-80}$ 
Disease management with calprotectin and TDM gained interest as from the late zeroes and it is therefore unlikely to have had major impact on the results of the studies in this thesis.

Novel treatment goal For decades, the primary treatment goal in IBD has been to induce and maintain clinical disease remission. ${ }^{81-82}$ Similarly, clinical remission has been the primary endpoint in nearly all large RCTs on therapeutic agents in IBD. In other words, the treatment paradigm has always been to control symptoms rather than bowel inflammation. The first evidence for a strong discrepancy between symptoms and bowel inflammation already dates back to 1994, when Cellier et al. observed a weak correlation between the symptom-driven CDAI score and the endoscopy-driven CDEIS score. ${ }^{83}$ These findings were later confirmed by several other studies. ${ }^{84-86}$ Under the previously discussed assumption that longstanding [subclinical] bowel inflammation ultimately results in irreversible bowel damage, it was hypothesized that aiming for an inflammation-free bowel may result in a lower risk of developing complicated or refractory disease. ${ }^{43}$ Current evidence for this hypothesis is weak and primarily derives from post-hoc analyses of large clinical trials, such as the TOP-DOWN ${ }^{87}$, ACCENT- $\left.\right|^{45}$ and EXTEND ${ }^{88,89}$ trials in CD and the ACT-trials ${ }^{50,90}$ in UC. Although new strategy trials are underway, such as the REACT2 study [ClinicalTrials.gov; NCT01698307], it is unlikely that such trials will provide a clear answer to the question whether striving for endoscopic remission can change the long-term disease outcome. After all, it is important to follow patients for a long period of time [preferably at least two years in order to assess structural bowel damage] and to acknowledge that some practical hurdles need to be taken. First, a universal, generally accepted definition of endoscopic remission must be stated, particularly in UC. ${ }^{91}$ Second, it is currently unknown whether we must strive for endoscopic [macroscopic] or histologic [microscopic] remission, acknowledging that the endoscopic view only addresses mucosal and not necessarily transmural healing. Third, we currently do not know how frequent endoscopic remission must be assessed when striving for an inflammation-free bowel. Last, future studies must assess whether biomarkers [e.g. fecal calprotectin ${ }^{74,75,92}$ ] and radiologic imaging ${ }^{93,94}$ are valid non-invasive proxies for endoscopic remission in daily practice.

\section{The future of IBD research: real-world epidemiology}

The increasing therapeutic armamentarium in combination with the heterogenous course and outcome of IBD make it increasingly difficult to select the right treatment at the right time for the right patient. Despite hard work in the fields of IBD genetics, pathophysiology and microbiota, it is still not possible to predict which patients will develop severe disease, which patients will respond best to a specific therapy and in which patient treatment can safely be 
de-escalated or stopped. Other knowledge gaps reside in the discrepancy between treatment effects in RCTs [efficacy] as compared to daily practice [effectiveness] and in the effectiveness of medical therapy in specific subgroups of patients who are often excluded in trials [e.g. elderly, young women, patients with moderate to severe comorbidities]. As previously stated, it is unlikely that RCTs will fill these gaps, given the heterogeneity of both IBD and the patient population. Analyzing large real-world data [RWD] sets with clinical, biochemical, pharmacological, histopathological, genetical and microbial data, preferably with direct linkage to electronic patient records and E-health tools, may prove invaluable in the process of establishing a personalized therapeutic strategy in IBD. Registration and analysis of RWD for decision moments in guidelines where knowledge gaps exist is crucial to make the transition from evidence that a particular intervention works to evidence for whom and when the intervention works.

In conclusion, the temporal improvements in the disease outcome of IBD, as described in the current thesis, must be ascribed to an interplay of many factors, including, but not limited to, changes in medical therapy. Insight in the question whether medical therapy can change the long-term outcome of IBD if it is given as part of a more tight or aggressive treatment strategy, is one of the main unmet needs in IBD management. Several new treatment strategies have been proposed, but the available RCTs lack external validity and trials inevitably go beyond the heterogeneity of IBD and diversity in patient population. Second, as most new treatment strategies aim to treat the disease more aggressive, especially early in disease course, the risk of overtreatment looms. A personalized therapeutic strategy is desirable and hopefully RWD analyses will fill current knowledge gaps. Meanwhile, the ultimate challenge in IBD management and research remains: treating the right patient with the right drug at the right time. 


\section{References}

1. Kaplan GG. Pitfalls and perils of using administrative databases to evaluate the incidence of inflammatory bowel disease overtime. Inflamm Bowel Dis 2014;20:1777-9.

2. Gower-Rousseau C, Savoye G, Colombel $J F$, Peyrin-Biroulet L. Are we improving disease outcomes in IBD? A view from the epidemiology side. Gut 2014;63:1529-30.

3. van den Heuvel TR, Pierik MJ, Jonkers DM. On the external validity of epidemiologic data from hospital-based IBD cohorts. J Crohns Colitis 2016.

4. Suissa S. Immortal time bias in pharmaco-epidemiology. Am J Epidemiol 2008;167:492-9.

5. Suissa S. Immortal time bias in observational studies of drug effects. Pharmacoepidemiol Drug Saf 2007;16:241-9.

6. Lakatos PL, Golovics PA, David G, et al. Has there been a change in the natural history of crohn's disease? Surgical rates and medical management in a population-based inception cohort from western hungary between 1977-2009. Am J Gastroentero/ 2012;107:579-88.

7. Picco MF, Zubiaurre I, Adluni M, et al. Immunomodulators are associated with a lower risk of first surgery among patients with non-penetrating nonstricturing crohn's disease. Am J Gastroentero/2009;104:2754-9.

8. Peyrin-Biroulet L, Oussalah A, Williet N, et al. Impact of azathioprine and tumour necrosis factor antagonists on the need for surgery in newly diagnosed Crohn's disease. Gut 2011;60:930-6.

9. Safroneeva E, Vavricka SR, Fournier N, et al. Impact of the early use of immunomodulators or TNF antagonists on bowel damage and surgery in Crohn's disease. Aliment Pharmacol Ther 2015;42:977-89.

10. Kariyawasam VC, Selinger $C P$, Katelaris $\mathrm{PH}$, et al. Early use of thiopurines or methotrexate reduces major abdominal and perianal surgery in Crohn's disease. Inflamm Bowel Dis 2014;20:1382-90.

11. Qiu Y, Chen BL, Feng R, et al. Prolonged azathioprine treatment reduces the need for surgery in early Crohn's disease. J Gastroenterol Hepatol 2017.

12. Williet N, Pillot $C$, Oussalah $A$, et al. Incidence of and impact of medications on colectomy in newly diagnosed ulcerative colitis in the era of biologics. Inflamm Bowel Dis 2012;18:1641-6.

13. Chhaya V, Saxena S, Cecil E, et al. The impact of timing and duration of thiopurine treatment on colectomy in ulcerative colitis: A national populationbased study of incident cases between 1989-2009. Aliment Pharmacol Ther 2015;41:87-98.

14. Targownik LE, Suissa S. Understanding and avoiding immortal-time bias in gastrointestinal observational research. Am J Gastroentero/ 2015;110:164750.

15. Molodecky NA, Soon IS, Rabi DM, et al. Increasing incidence and prevalence of the inflammatory bowel diseases with time, based on systematic review. Gastroenterology 2012;142:46-54 e42; quiz e30.

16. Kok L, Elias SG, Witteman BJ, et al. Diagnostic accuracy of point-of-care fecal calprotectin and immunochemical occult blood tests for diagnosis of organic bowel disease in primary care: The cost-effectiveness of a decision rule for abdominal complaints in primary care (cedar) study. Clin Chem 2012;58:98998.

17. Spooren CE, Pierik MJ, Zeegers MP, et al. Review article: The association of diet 
with onset and relapse in patients with inflammatory bowel disease. Aliment Pharmacol Ther 2013;38:1172-87.

18. Mahid SS, Minor KS, Soto RE, et al. Smoking and inflammatory bowel disease: A meta-analysis. Mayo Clin Proc 2006;81:1462-71.

19. Soon IS, Molodecky NA, Rabi DM, et al. The relationship between urban environment and the inflammatory bowel diseases: A systematic review and metaanalysis. BMC Gastroenterol 2012;12:51.

20. Kaplan GG, Hubbard J, Korzenik J, et al. The inflammatory bowel diseases and ambient air pollution: A novel association. Am J Gastroenterol 2010;105:2412-9.

21. Ananthakrishnan AN, McGinley EL, Binion DG, Saeian K. Ambient air pollution correlates with hospitalizations for inflammatory bowel disease: An ecologic analysis. Inflamm Bowel Dis 2011;17:1138-45.

22. Salim SY, Kaplan GG, Madsen KL. Air pollution effects on the gut microbiota: A link between exposure and inflammatory disease. Gut Microbes 2014;5:215-9.

23. Heuvel T, Bijnens E, Verhaegh B, et al. P690 ambient air quality does not affect disease course in inflammatory bowel disease - a population based risk factor analysis using geographic information systems; 2017.

24. Maaser $\mathrm{C}$, Langholz E, Gordon H, et al. European crohn's and colitis organisation topical review on environmental factors in IBD J Crohns Colitis 2017;11:90520.

25. de Souza HSP, Fiocchi C, lliopoulos D. The IBD interactome: An integrated view of aetiology, pathogenesis and therapy. Nat Rev Gastroenterol Hepatol 2017; 14:739-49.

26. van der Valk ME, Mangen MJ, Leenders $M$, et al. Risk factors of work disability in patients with inflammatory bowel disease--a Cutch nationwide web-based survey: Work disability in inflammatory bowel disease. J Crohns Colitis 2014;8:590-7.

27. Romberg-Camps MJ, Bol Y, Dagnelie PC, et al. Fatigue and health-related quality of life in inflammatory bowel disease: Results from a population-based study in the Netherlands: The IBD-South Limburg cohort. Inflamm Bowel Dis 2010;16:2137-47.

28. Irvine EJ. Review article: Patients' fears and unmet needs in inflammatory bowe disease. Aliment Pharmacol Ther 2004;20 Suppl 4:54-9.

29. de Jong MJ, van der Meulen-de Jong AE, Romberg-Camps MJ, et al. Telemedicine for management of inflammatory bowel disease (myilBDoach): A pragmatic, multicentre, randomised controlled trial. Lancet 2017;390:959-68.

30. Robinson A, Thompson DG, Wilkin D, Roberts C, Northwest Gastrointestinal Research G. Guided self-management and patient-directed follow-up of ulcerative colitis: A randomised trial. Lancet 2001;358:976-81.

31. Ponsioen CY, de Groof EJ, Eshuis EJ, et al. Laparoscopic ileocaecal resection versus infliximab for terminal ileitis in crohn's disease: A randomised controlled, open-label, multicentre trial. Lancet Gastroenterol Hepatol 2017:2:785-92.

32. Gomollon F, Dignass A, Annese V, et al. 3rd european evidence-based consensus on the diagnosis and management of crohn's disease 2016: Part 1: Diagnosis and medical management. J Crohns Colitis 2017;11:3-25.

33. Rungoe $\mathrm{C}$, Langholz $\mathrm{E}$, Andersson $\mathrm{M}$, et al. Changes in medical treatment and surgery rates in inflammatory bowel disease: A nationwide cohort study 1979-2011. Gut2014;63:1607-16.

34. Ramadas AV, Gunesh S, Thomas GA, et al. Natural history of Crohn's disease in a population-based cohort from Cardiff 
[1986-2003]: A study of changes in medical treatment and surgical resection rates. Gut 2010;59:1200-6.

35. Jess $T$, Riis $L$, Vind I, et al. Changes in clinical characteristics, course, and prognosis of inflammatory bowel disease during the last 5 decades: A populationbased study from Copenhagen, Denmark. Inflamm Bowel Dis 2007;13:481-9.

36. van der Valk ME, Mangen MJ, Leenders $M$, et al. Healthcare costs of inflammatory bowel disease have shifted from hospitalisation and surgery towards anti-TNFalpha therapy: Results from the coin study. Gut 2014;63:72-9.

37. Burisch J, Jess T, Martinato M, Lakatos $P L$, EpiCom E. The burden of inflammatory bowel disease in Europe. $J$ Crohns Colitis 2013;7:322-37.

38. Targownik LE, Singh $H$, Nugent $Z$, Bernstein $\mathrm{CN}$. The epidemiology of colectomy in ulcerative colitis: Results from a population-based cohort. Am J Gastroentero/2012;107:1228-35.

39. Samuel S, Ingle SB, Dhillon $S$, et al. Cumulative incidence and risk factors for hospitalization and surgery in a population-based cohort of ulcerative colitis. Inflamm Bowel Dis 2013;19:1858-66.

40. Rieder F, Zimmermann EM, Remzi FH, Sandborn WJ. Crohn's disease complicated by strictures: A systematic review. Gut 2013;62:1072-84.

41. Scharl M, Rogler G. Pathophysiology of fistula formation in Crohn's disease. World J Gastrointest Pathophysiol 2014;5:205-12.

42. Lakatos PL, David G, Pandur T, et al. IBD in the elderly population: Results from a population-based study in Western Hungary, 1977-2008. J Crohns Colitis 2011;5:5-13.

43. Peyrin-Biroulet L, Sandborn W, Sands $\mathrm{BE}$, et al. Selecting therapeutic targets in inflammatory bowel disease (stride):
Determining therapeutic goals for treatto-target. Am J Gastroenterol 2015;110:1324-38

44. Allen PB, Peyrin-Biroulet L. Moving towards disease modification in inflammatory bowel disease therapy. Curr Opin Gastroentero/ 2013;29:397404.

45. Hanauer SB, Feagan BG, Lichtenstein $\mathrm{GR}$, et al. Maintenance infliximab for crohn's disease: The ACCENT I randomised trial. Lancet 2002;359:1541-9.

46. Hanauer SB, Sandborn WJ, Rutgeerts P, et al. Human anti-tumor necrosis factor monoclonal antibody (adalimumab) in Crohn's disease: The CLASSIC-I trial. Gastroenterology 2006;130:323-33; quiz 591.

47. Sandborn WJ, Hanauer SB, Rutgeerts $P$, et al. Adalimumab for maintenance treatment of Crohn's disease: Results of the classic ii trial. Gut 2007;56:1232-9.

48. Colombel JF, Sandborn WJ, Rutgeerts P, et al. Adalimumab for maintenance of clinical response and remission in patients with Crohn's disease: The charm trial. Gastroenterology 2007;132:5265.

49. Sandborn WJ, van Assche G, Reinisch W, et al. Adalimumab induces and maintains clinical remission in patients with moderate-to-severe ulcerative colitis. Gastroenterology 2012;142:257-65 e1-3.

50. Rutgeerts P, Sandborn WJ, Feagan BG, et al. Infliximab for induction and maintenance therapy for ulcerative colitis. N Eng/ J Med 2005;353:246276.

51. Reinisch W, Sandborn WJ, Rutgeerts P, et al. Long-term infliximab maintenance therapy for ulcerative colitis: The act-1 and -2 extension studies. Inflamm Bowel Dis 2012;18:201-11.

52. Biemans V, Van Der Meulen A, Van Der Woude CJ, et al. Mo1896-Ustekinumab 
for Crohn's disease: A nationwide reallife observational cohort study (ICC case series). Gastroenterology 2018;154:S842-S-3.

53. Bukhari $M$, Wiles $N$, Lunt $M$, et al. Influence of disease-modifying therapy on radiographic outcome in inflammatory polyarthritis at five years: Results from a large observational inception study. Arthritis Rheum 2003;48:46-53.

54. Egsmose C, Lund B, Borg G, et al. Patients with rheumatoid arthritis benefit from early 2 nd line therapy: 5 year followup of a prospective double blind placebo controlled study. J Rheumatol 1995:22:2208-13.

55. Fries JF, Williams CA, Morfeld D, et al. Reduction in long-term disability in patients with rheumatoid arthritis by disease-modifying antirheumatic drugbased treatment strategies. Arthritis Rheum 1996;39:616-22.

56. Lard LR, Visser H, Speyer I, et al. Early versus delayed treatment in patients with recent-onset rheumatoid arthritis: Comparison of two cohorts who received different treatment strategies. Am J Med 2001;111:446-51.

57. Sokka T, Möttönen T, Hannonen P. Disease-modifying anti-rheumatic drug use according to the sawtooth treatment strategy improves the functional outcome in rheumatoid arthritis: Results of a long-term follow-up study with review of the literature. Rheumatology 2000;39:34-42.

58. van der Heide A, Jacobs JW, Bijlsma JW, et al. The effectiveness of early treatment with "second-line" antirheumatic drugs a randomized, controlled trial. Ann Int Med 1996;124:699-707.

59. Mottonen $\mathrm{T}$, Hannonen $\mathrm{P}$, Korpela M, et al. Delay to institution of therapy and induction of remission using single-drug or combination-disease-modifying antirheumatic drug therapy in early rheumatoid arthritis. Arthritis Rheum 2002;46:894-8.

60. Breedveld FC, Weisman MH, Kavanaugh $A F$, et al. The premier study: A multicenter, randomized, double-blind clinical trial of combination therapy with adalimumab plus methotrexate versus methotrexate alone or adalimumab alone in patients with early, aggressive rheumatoid arthritis who had not had previous methotrexate treatment. Arthritis Rheum 2006;54:26-37.

61. Goekoop-Ruiterman YP, de VriesBouwstra JK, Allaart CF, et al. Clinical and radiographic outcomes of four different treatment strategies in patients with early rheumatoid arthritis (the best study): A randomized, controlled trial. Arthritis Rheum 2005;52:3381-90.

62. Panes J, Lopez-Sanroman A, Bermejo F, et al. Early azathioprine therapy is no more effective than placebo for newly diagnosed Crohn's disease. Gastroenterology 2013;145:766-74 e1.

63. Cosnes J, Bourrier A, Laharie D, et al. Early administration of azathioprine vs conventional management of crohn's disease: A randomized controlled trial. Gastroenterology 2013;145:758-65 e2; quiz e14-5.

64. Matsumoto T, Motoya S, Watanabe K, et al. Adalimumab monotherapy and a combination with azathioprine for crohn's disease: A prospective, randomized trial. J Crohns Colitis 2016;10:1259-66.

65. D'Haens G, Baert F, van Assche G, et al. Early combined immunosuppression or conventional management in patients with newly diagnosed Crohn's disease: An open randomised trial. Lancet 2008;371:660-7.

66. Colombel JF, Sandborn WJ, Reinisch W, et al. Infliximab, azathioprine, or combination therapy for Crohn's disease. N Eng/ J Med2010;362:1383-95. 
67. Panaccione R, Ghosh S, Middleton S, et al. Combination therapy with infliximab and azathioprine is superior to monotherapy with either agent in ulcerative colitis. Gastroenterology 2014; 146:392-400 e3.

68. Khanna R, Bressler B, Levesque BG, et al. Early combined immunosuppression for the management of crohn's disease (REACT): A cluster randomised controlled trial. Lancet 2015;386:182534.

69. Grigor C, Capell H, Stirling A, et al. Effect of a treatment strategy of tight control for rheumatoid arthritis (the TICORA study): A single-blind randomised controlled trial. Lancet 2004;364:2639.

70. Schipper LG, van Hulst LT, Grol R, et al. Meta-analysis of tight control strategies in rheumatoid arthritis: Protocolized treatment has additional value with respect to the clinical outcome. Rheumatology (Oxford) 2010;49:215464.

71. Tibble JA, Sigthorsson G, Bridger S, Fagerhol MK, Bjarnason I. Surrogate markers of intestinal inflammation are predictive of relapse in patients with inflammatory bowel disease. Gastroenterology 2000;119:15-22.

72. D'Inca R, Dal Pont E, Di Leo V, et al. Can calprotectin predict relapse risk in inflammatory bowel disease? Am J Gastroentero/2008;103:2007-14.

73. Sands BE. Biomarkers of inflammation in inflammatory bowel disease. Gastroenterology 2015;149:1275-85 e2.

74. D'Haens G, Ferrante M, Vermeire S, et al. Fecal calprotectin is a surrogate marker for endoscopic lesions in inflammatory bowel disease. Inflamm Bowel Dis 2012;18:2218-24.

75. Roseth AG, Aadland E, Grzyb K. Normalization of faecal calprotectin: A predictor of mucosal healing in patients with inflammatory bowel disease. Scand J Gastroentero/ 2004;39:1017-20.

76. Colombel JF, Panaccione R, Bossuyt P, et al. Effect of tight control management on crohn's disease (CALM): A multicentre, randomised, controlled phase 3 trial. Lancet 2017.

77. Yanai $\mathrm{H}$, Lichtenstein L, Assa A, et al. Levels of drug and antidrug antibodies are associated with outcome of interventions after loss of response to infliximab or adalimumab. Clin Gastroenterol Hepato/ 2015;13:522-30 e2.

78. Roblin X, Rinaudo M, Del Tedesco E, et al. Development of an algorithm incorporating pharmacokinetics of adalimumab in inflammatory bowel diseases. Am J Gastroenterol 2014;109:1250-6.

79. Vande Casteele N, Ferrante M, Van Assche G, et al. Trough concentrations of infliximab guide dosing for patients with inflammatory bowel disease. Gastroenterology 2015;148:1320-9 e3.

80. D'Haens $\mathrm{G}$, Vermeire $\mathrm{S}$, Lambrecht $\mathrm{G}$, et al. Increasing infliximab dose based on symptoms, biomarkers, and serum drug concentrations does not increase clinical, endoscopic, and corticosteroid-free remission in patients with active luminal Crohn's disease. Gastroenterology 2018; 154:1343-51 e1.

81. Dignass A, Lindsay JO, Sturm A, et al. Second european evidence-based consensus on the diagnosis and management of ulcerative colitis part 2: Current management. J Crohns Colitis 2012;6:991-1030

82. Dignass A, Van Assche G, Lindsay JO, et al. The second european evidence-based consensus on the diagnosis and management of Crohn's disease: Current management. J Crohns Colitis 2010;4:28-62.

83. Cellier C, Sahmoud T, Froguel E, et al. Correlations between clinical activity, 
endoscopic severity, and biological parameters in colonic or ileocolonic crohn's disease. A prospective multicentre study of 121 cases. The groupe d'etudes therapeutiques des affections inflammatoires digestives. Gut 1994;35:231-5.

84. Peyrin-Biroulet L, Reinisch W, Colombel JF, et al. Clinical disease activity, creactive protein normalisation and mucosal healing in Crohn's disease in the sonic trial. Gut 2014;63:88-95.

85. Efthymiou A, Viazis N, Mantzaris G, et al. Does clinical response correlate with mucosal healing in patients with crohn's disease of the small bowel? A prospective, case-series study using wireless capsule endoscopy. Inflamm Bowel Dis 2008;14:1542-7.

86. Sipponen T, Savilahti E, Kolho KL, et al. Crohn's disease activity assessed by fecal calprotectin and lactoferrin: Correlation with Crohn's disease activity index and endoscopic findings. Inflamm Bowel Dis 2008;14:40-6.

87. Baert F, Moortgat L, Van Assche G, et al. Mucosal healing predicts sustained clinical remission in patients with earlystage Crohn's disease. Gastroenterology 2010;138:463-8; quiz e10-1.

88. Rutgeerts $\mathrm{P}$, Diamond $\mathrm{RH}$, Bala $\mathrm{M}$, et al. Scheduled maintenance treatment with infliximab is superior to episodic treatment for the healing of mucosal ulceration associated with Crohn's disease. Gastrointest Endosc 2006;63:433-42; quiz 64.

89. Colombel JF, Rutgeerts PJ, Sandborn WJ, et al. Adalimumab induces deep remission in patients with Crohn's disease. Clin Gastroenterol Hepatol 2014; 12:414-22 e5.

90. Colombel JF, Rutgeerts P, Reinisch W, et al. Early mucosal healing with infliximab is associated with improved long-term clinical outcomes in ulcerative colitis. Gastroenterology 2011;141:1194-201.
91. D'Haens G, Sandborn WJ, Feagan BG, et al. A review of activity indices and efficacy end points for clinical trials of medical therapy in adults with ulcerative colitis. Gastroenterology 2007;132:763-86.

92. Boon GJ, Day AS, Mulder CJ, Gearry RB. Are faecal markers good indicators of mucosal healing in inflammatory bowel disease? World J Gastroenterol 2015:21:11469-80.

93. Kaushal P, Somwaru AS, Charabaty A, Levy AD. Mr enterography of inflammatory bowel disease with endoscopic correlation. Radiographics 2017;37:116-31

94. Moy MP, Kaplan JL, Moran CJ, et al. MR enterographic findings as biomarkers of mucosal healing in young patients with Crohn disease. AJR Am J Roentgenol 2016:1-7 




\section{SUMMARY}

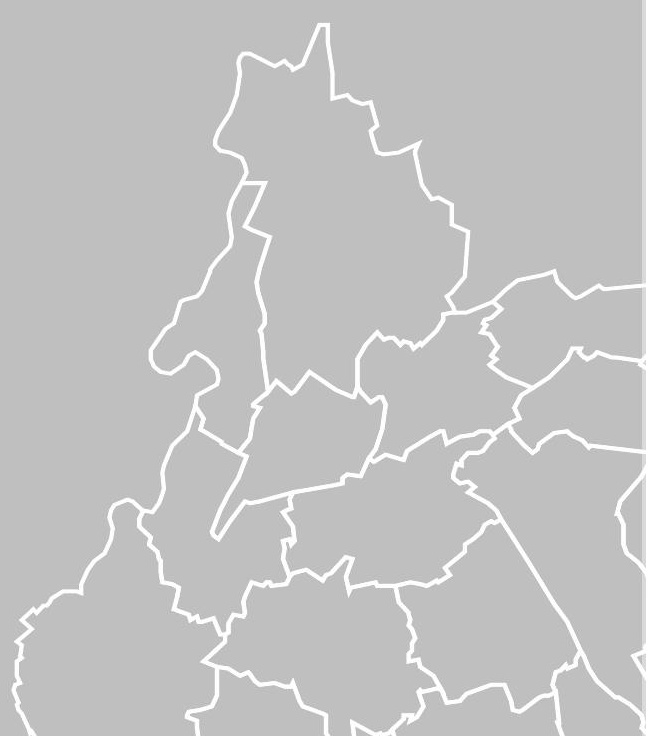




\section{Summary}

Inflammatory bowel disease [IBD] is a chronic inflammatory disease of the gastro-intestinal tract. Patients suffer from symptoms like abdominal pain, diarrhea, bloody stools, urgency and fatigue. Thereby, the disease often has large impact on daily life. Two main disease entities can be distinguished: Crohn's disease [CD] and ulcerative colitis [UC]. In CD, inflammation can be present throughout the gastrointestinal tract, although the terminal ileum and colon are frequently affected. Inflammation is not limited to the mucosal layer and as such, CD patients are prone to develop fistula tracts and fibrotic strictures. In UC, inflammation is limited to the superficial mucosal layer, starts at the anal margin and spreads proximally in a continuous fashion. The disease courses of both CD and UC are heterogenous and characterized by periods of active disease, alternated with periods of disease remission. Both medical therapy and surgery are used in disease management, though choosing the optimal treatment strategy for an individual patient is challenging. Over the past decades, several changes in diagnosis and management have been introduced, which may have impacted the epidemiology and outcome of IBD. For instance, novel drugs have been registered [e.g. anti-tumor necrosis factor $\alpha$ [anti-TNF $\alpha$ ] therapy] and treatment strategies have changed [e.g. 'accelerated step-

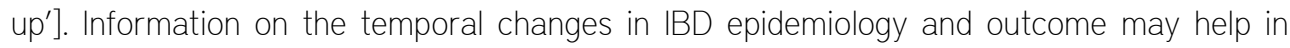
resource planning and allocation and retrieving insight in the real-world effectiveness of IBD medication.

\section{Epidemiology of IBD in South Limburg}

In the first part of the thesis, the epidemiology of IBD in South Limburg was discussed.

In Chapter 2, the basis for all subsequent studies of this thesis was presented: the cohort profile of the population-based IBD South Limburg [IBDSL] cohort. As from 1991, all newly-diagnosed adult IBD patients in the South Limburg area have been registered and detailed clinical data are being collected and updated biannually. As from 2011, the cohort has been scaled-up to a biobank, including samples of serum plasma, DNA and feces, to employ marker and mechanistic studies. Population-based cohort studies aim to include all patients in a specific population. By using a multifaceted approach, including prospective inclusion, review of hospital administrations and a review of the national pathology databank PALGA, we strived for high cohort completion. We performed a crosscheck with regional general practitioners, which showed that 93\% of the IBD population of South Limburg is currently captured. As of 2012, the cohort comprised 2837 IBD patients [1162 CD and 1675 UC patients]. By time of conducting the studies in this thesis, follow-up was complete up to 2014 [CD] and 2011 [UC]. The IBDSL cohort provided a unique opportunity to study temporal 
changes in the epidemiology and outcome of IBD in an unselected, large population in the Netherlands.

To assess the current burden of IBD on healthcare and society, we assessed temporal changes in its incidence, prevalence and mortality, which were discussed in Chapter 3. Between 1991 and 2010, the incidences of both CD and UC increased markedly; from 5.84 to 17.49 per 100,000 in CD and from 11.67 to 21.47 per 100,000 in UC. By using Joinpoint regression analyses, we found that the incidences increased as of the late nineties and are probably still increasing. The IBD prevalence in South Limburg was found to be 830 per 100,000 [4186 prevalent cases] and, via extrapolation, the nationwide prevalence was estimated at 613 per 100,000 inhabitants [80,627 prevalent cases]. This is higher than the previously estimated prevalence $[55,000]$. Part of the increase may be explained by former underdiagnosis, given the incidence increase of patients with limited and uncomplicated disease at diagnosis. However, environmental factors are also likely to play a role, given the short time span in which the increase has taken place. The findings of this study illustrate the increasing importance of IBD for healthcare and society and underline the importance of studies on IBD etiology to come to preventive measures.

Elderly patients are often underrepresented in clinical trials, although insight in the epidemiology and disease course of elderly with IBD is of increasing importance, given the demographic phenomenon of population ageing. Therefore, in Chapter 4, the incidence, medical management and disease outcome of elderly IBD patients were assessed. We found that in elderly the IBD incidence has also increased: from 2.51 [1991] to 6.23 [2010] per 100,000 for CD and from 9.20 to 17.43 per 100,000 for UC. Together with population ageing, this trend will result in a strong increase in the prevalence of elderly patients with IBD. We observed that the medical management differed, in that elderly, as compared to adultonset disease, less often used immunomodulators [61.8\% vs. $77.1 \%$ in CD and $22.8 \%$ vs. $35.4 \%$ in UC] and anti-TNF $\alpha$ agents [25.1\% vs. $55.1 \%$ in CD and $7.8 \%$ vs. $18.0 \%$ in UC]. This has, however, not resulted in a worse long-term disease outcome, since the 5 -year risks of phenotype progression [CD: 19.8\% vs. 24.2\%], cumulative corticosteroid use [CD: 22.1 vs. 15.5 days per patient year] and surgery [CD: $29.2 \%$ vs. $27.1 \%$, UC: $7.0 \%$ vs. $5.8 \%$ ] were similar between groups. The hospitalization rate was similar in CD [49.0\% vs. 53.4\%], but higher in UC in the elderly population [26.7\% vs. 17.9\%], which can be attributed to a more frequent in-hospital diagnosis of UC. Also supportive for a difference in the diagnostic approach or presentation is the observation that more elderly- than adult-onset CD patients underwent surgery at diagnosis [14.1\% vs $7.1 \%$ ]. Our findings confirm that elderly-onset IBD is of increasing importance and we provided physicians insight in the medical management and outcome of this population. 


\section{Impact of advances in IBD management: new era, better future?}

In the second part of the thesis, we evaluated the temporal changes in disease outcome, with special focus on the concomitant advances in medical therapy. The long-term outcome was assessed in terms requiring hospitalization, undergoing surgery, cumulative corticosteroid use and the risk of developing CD-related complications [i.e. phenotype progression].

For CD [Chapters 5 and 7 ], we found that immunomodulators were earlier and more frequently prescribed [5-year exposure: $30.6 \%$ in era 1991-1998 and $70.8 \%$ in era 20062011] and we came to similar conclusions regarding anti-TNFa therapy [3.1\% and $41.2 \%$, respectively]. The corticosteroid exposure rate remained stable [54.0\%]. In parallel, the risks of hospitalization [65.9\% and 44.2\%] and surgery [42.9\% and 17.4\%] decreased markedly. These declines could partly be explained by events avoided shortly after disease diagnosis, pointing at differences in the diagnostic process and initial disease management. Moreover, the cumulative corticosteroid use after the first year decreased: from a median of 198 days [1991-1998] to 0 days [2006-2011]. The risk of phenotype progression, however, remained unchanged [21.6\% after 5 years]. By using advanced statistics, we assessed the relation between medication use and long-term outcome measures and only found significant associations between the use of immunomodulator and anti-TNF $\alpha$ therapy and corticosteroid use. Thereby, our findings illustrate that improvements in other outcome parameters [i.e. hospitalization, surgery] are probably predominantly related to other factors than advances in medical therapy, such as novel insights in the indications for hospital admission and surgery and changes in disease management [e.g. tight control]. Another invalidating complication of CD are perianal fistulas. In Chapter 8 , the time trends in the incidence of perianal disease in $\mathrm{CD}$ were assessed. The 5 -year risk of developing a perianal fistula was stable over time [11.6\%], despite the fact that an increasing number of CD patients was already exposed to immunomodulators and anti-TNFa therapy prior to the fistula diagnosis. After fistula diagnosis, more than half of the patients were on biological therapy. Nevertheless, the fistula recurrence rate was also found to be stable over time [25.7\% after 5 years]. Of note, a concomitant trend of more fistula awareness, including a more frequent use of magnetic resonance imaging [MRI], may have affected the fistula incidence and recurrence rates in more recent years. Our findings underline the lasting need for improving medical and surgical treatment for fistulizing $C D$, including strategies to reduce the risk of developing fistulizing disease.

For UC [Chapters 6 and 7 ], we also found an increasing exposure rate for immunomodulators [8.1\% in era 1991-1997 and 21.7\% in era 2006-2010, 5 years after diagnosis] and a shorter time to first prescription. In line, anti-TNF $\alpha$ agents were more frequently and earlier used [0\% in era 1991-1997 and 10.6\% in era 2006-2010]. As in CD, the corticosteroid exposure rate was stable [31.4\%]. The hospitalization rate was found to be 
stable over time [20.0\%], whereas the 5-year colectomy rate decreased from $7.5 \%$ in the first to $4.1 \%$ in the most-recent era. The latter was found to be caused by avoided colectomies within the first six months of disease [i.e. early colectomy]. The risk of undergoing surgery thereafter was even found to be stable over time. As in CD, these observations suggest that changes in the diagnostic process and initial disease management play a role. A strong medication effect is not expected, because immunomodulator and anti-TNF $\alpha$ exposure rates were low within the time frame of early colectomy. Furthermore, the cumulative corticosteroid use after the first year decreased [median 114 days in 1991-1998 to 94 days in 20062011], and a significant association was found between immunomodulator and anti-TNF $\alpha$ use and the need for corticosteroids.

Last, in Chapter 9, the long-term real-world effectiveness of infliximab [IFX] and adalimumab [ADA] was assessed in CD patients and a comparative effectiveness analysis was conducted. Anti-TNF $\alpha$ agents are more frequently and earlier used, in particular in patients with characteristics of expected severe disease [e.g. colonic or ileocolonic CD, perianal disease, upper gastrointestinal involvement and smokers]. Both IFX and ADA showed durable response with corticosteroid-free response rates of $67.1 \%$ [IFX] and 70.1\% [ADA] after five years of treatment in primary responders. No significant difference in effectiveness was observed between the two agents. Nearly half of the patients restarted anti-TNF $\alpha$ therapy after discontinuation and about one-third of this population showed durable response to second-line anti-TNF $\alpha$ therapy, regardless of the reason for prior anti-TNF $\alpha$ cessation. Most patients were on combination therapy [57.6\% for IFX and 35.7\% for ADA]. We did not find higher response rates in patients on combination therapy as compared to monotherapy, hereby acknowledging possible residual confounding by indication due to the strong association between combination therapy and disease severity. Our findings did not show a difference in comparative effectiveness between IFX and ADA, thereby favoring agent selection based on the individual preferences of patient and physician.

Finally, in Chapter 10, the conclusions of all studies were put into perspective. First, the epidemiological challenges in IBD research were discussed, including the relevance of selecting an appropriate study design and the importance of reducing the effects of immune-time bias in analyses on the association between medication use and disease outcome. Second, the implications of the rising IBD incidence were outlined, including suggestions for future-proof $\mathrm{IBD}$ care and targets for further research. Third, our observations on the temporal changes in medical therapy and long-term outcome of IBD were discussed in detail, including an overview of several novel treatment strategies. We concluded with the message that large real-world studies are needed to provide answers to clinically relevant questions on the optimal, personalized treatment strategy of IBD. 



\section{SAMENVATTING}

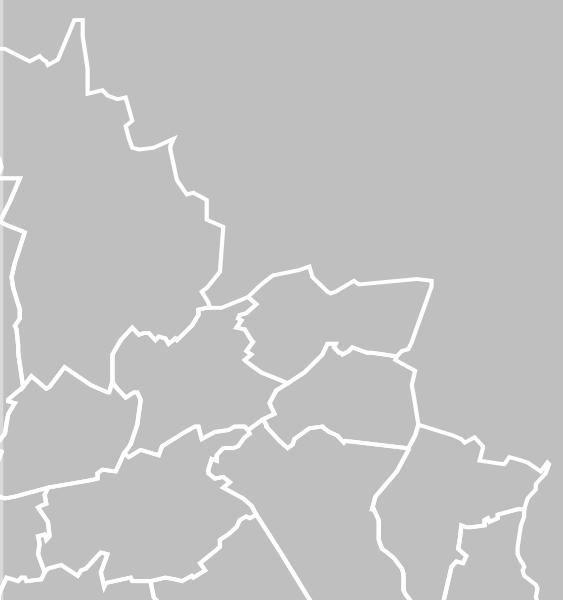




\section{Samenvatting}

Inflammatoire darmziekten [IBD] zijn chronische ontstekingsziekten van het maagdarmkanaal, waarbij patiënten klachten hebben zoals buikpijn, diarree, bloederige ontlasting, aandrang en chronische vermoeidheid. De impact op het dagelijks leven is groot. IBD is een parapluterm voor twee ziektebeelden: de ziekte van Crohn [ZvC] en colitis ulcerosa [CU]. In het geval van de ZvC kan de ontsteking zich in het gehele maag-darmkanaal bevinden, maar het terminale ileum en het colon zijn wel voorkeurslokalisaties. De ontsteking kan zich uitbreiden tot diep in de darmwand, waardoor er vernauwingen [stricturen] en fisteltrajecten kunnen ontstaan. Bij colitis ulcerosa, daarentegen, beperkt de ontsteking zich tot de mucosalaag en komt de ziekte alleen in het colon voor. De ontsteking begint vaak in het rectum en kan zich proximaal uitbreiden. In de behandeling van IBD is plaats voor zowel [immunosuppressieve] medicatie als chirurgie. Het bepalen van de optimale behandelstrategie voor een individuele patiënt is echter lastig, waarbij zowel onder- als overbehandeling moet worden voorkomen. In de afgelopen decennia hebben er veranderingen plaatsgevonden in de behandeling van patiënten met IBD. Zo zijn de anti-tumor necrosis factor $\alpha$ [anti-TNF $\alpha$ ] preparaten beschikbaar gekomen aan het einde van jaren negentig. Daarnaast zijn ook de behandelstrategieën en -inzichten veranderd; zo wordt er sneller opgeschaald naar sterkere immunosuppressieve middelen. Inzicht krijgen in de veranderingen in het vóórkomen van IBD draagt bij aan een goede planning en toewijzing van zorgproducten. Aan de andere kant draagt inzicht in veranderingen in de lange-termijn uitkomsten van IBD bij aan de kennis over de effectiviteit van geneesmiddelen in de dagelijkse praktijk.

\section{Epidemiologie van inflammatoire darmziekten in Zuid-Limburg}

In het eerste deel van dit proefschrift werd de epidemiologie van IBD in Zuid-Limburg bestudeerd.

In Hoofdstuk 2 werd het cohortprofiel van het IBD Zuid-Limburg [IBDZL] cohort besproken. Het IBDZL-cohort is een populatie-gebaseerd cohort van IBD-patiënten welke de basis heeft gevormd voor deze thesis. In dit cohort worden sinds 1991 alle nieuwgediagnosticeerde IBD-patiënten geregistreerd en wordt op een gestructureerde manier gedetailleerde informatie verzameld over het ziektebeloop. Deze informatie wordt om het jaar geüpdate. Sinds 2011 worden ook biomaterialen verzameld [serum, DNA, ontlasting, uitademingslucht], zodat ook basaal-wetenschappelijk kan worden uitgevoerd naar onder andere biomarkers voor ziekteactiviteit en de rol van microbiota. Het doel van populatiegebaseerde cohortstudies is om alle patiënten in een populatie te identificeren en bestuderen. Door gebruik te maken van meerdere bronnen, inclusief prospectieve patiëntinclusie, ziekenhuisregistraties en de pathologie-database PALGA, hebben wij gestreefd zoveel 
mogelijk IBD-patiënten te identificeren. Een cross-check met regionale huisartspraktijken toonde aan dat 93\% van de IBD-populatie in Zuid-Limburg is geïncludeerd in het IBDZLcohort. In 2012 omvatte het cohort 2837 patiënten, waarvan 1162 ZvC patiënten en 1675 CU-patiënten. Het einde van de follow-up van de studies in deze thesis was 2011 voor CU en 2014 voor ZvC. De grootte van het cohort en de lange looptijd creëerden een unieke mogelijkheid om veranderingen in de epidemiologie en lange-termijn uitkomsten te bestuderen in grote populatie Nederlandse IBD-patiënten.

In Hoofdstuk 3 beschreven we veranderingen in de incidentie, prevalentie en mortaliteit van IBD, waarmee inzicht is verkregen in de impact van IBD op de Nederlandse gezondheidszorg en samenleving. De incidenties van zowel de ZvC als van CU zijn tussen 1991 en 2010 sterk gestegen. In het geval van de ZvC zagen we een toename van 5,84 naar 17,49 per 100.000 inwoners en in het geval van CU zagen we een toename van 11,67 naar 21,47 per 100.000. Door gebruik te maken van Joinpoint regressiemodellen ontdekten we dat de incidentie pas sinds het einde van de jaren negentig aan het stijgen is en dat deze stijging waarschijnlijk nog steeds gaande is. De prevalentie van IBD in Zuid-Limburg werd geschat op 830 per 100,000 inwoners [4186 patiënten]. Via extrapolatie werd eveneens een schatting gemaakt van de nationale prevalentie en deze kwam uit op 613 per 100.000 inwoners [80.627 patiënten]. Deze schatting is beduidend hoger dan de eerdere schatting [55.000]. De incidentiestijging kan waarschijnlijk gedeeltelijk worden verklaard door een onderdiagnose in het verleden, aangezien er vooral een stijging werd gezien van patiënten met een beperkte en ongecompliceerde ziekte. Waarschijnlijk spelen ook omgevingsfactoren een rol; de tijd waarin de stijging heeft plaatsgevonden is te kort om een effect van genetische factoren te verwachten. De bevindingen van deze studie hebben inzicht gegeven in de druk die IBD uitoefent op de maatschappij en illustreren de noodzaak om te komen tot preventieve maatregelen.

Ouderen zijn vaak ondervertegenwoordigd in gerandomiseerde studies. Echter, het verkrijgen van inzicht in het ziekteverloop in deze populatie is juist van toegevoegde waarde, rekening houdend met de toenemende vergrijzing. Om deze reden onderzochten we in Hoofdstuk 4 de incidentie, de medicamenteuze behandeling en de lange-termijn uitkomsten van IBD in een populatie patiënten met een diagnose op oudere leeftijd [ $\geq 60$ jaar]. De incidentie bleek ook stijgende in de oudere populatie: van 2,51 per 100.000 in 1991 naar 6,23 per 100.000 in 2010 in het geval van de ZvC en van 9,20 naar 17,43 per 100.000 in het geval van CU. Gelet op de vergrijzing van de samenleving betekent dit dat het aantal oudere patiënten met IBD sterk zal gaan toenemen. Ook was te zien dat de medicamenteuze behandeling op enkele punten verschilde van de volwassenen. Zo werden er bij IBD op oudere leeftijd minder vaak immunomodulatoren gebruikt [61,8\% t.o.v. $77,1 \%$ in ZvC en $22,8 \%$ t.o.v. $35,4 \%$ in CU] en ook minder vaak anti-TNF $\alpha$ middelen toegepast [25,1\% t.o.v. 55,1\% in ZvC 
en $7,8 \%$ t.o.v. $18,0 \%$ in CU] in vergelijking met de jongvolwassen populatie. Desondanks bleken de lange-termijn uitkomsten gelijk aan de groep IBD-patiënten met een diagnose op jongvolwassen leeftijd. De 5-jaars risico's van fenotype progressie [19,8\% t.o.v. 24,2\%] en chirurgie [29,2\% t.o.v. $27,1 \%$ voor ZvC en 7,0\% t.o.v. 5,8\% voor CU] waren gelijk tussen de twee groepen, evenals het cumulatieve corticosteroïd gebruik [22,1 dagen t.o.v. 15,5 dagen per patientjaar in de ZvC]. Alleen het risico op een ziekenhuisopname lag iets hoger bij oudere dan bij jongvolwassen CU-patiënten [26,7\% t.o.v. 17,9\%], hetgeen vooral bleek te berusten op een hoger aantal ziekenhuisopnames ten tijde van de diagnose van de ziekte. Bij de ZvC werd ook een verschil gezien tussen jongvolwassenen en ouderen rondom diagnosestelling: het risico op vroege darmchirurgie lag beduidend hoger bij de ouderen [14,1\%] dan bij de jong-volwassenen [7,1\%]. Onze resultaten illustreren dat IBD op oudere leeftijd een toenemend fenomeen is en dat er meer inzicht moet komen in de effecten en bijwerkingen van behandelingen en in de lange-termijn uitkomsten in deze specifieke populatie.

\section{Impact van veranderingen in IBD management: nieuw tijdperk, betere toekomst?}

In het tweede deel van het proefschrift keken we naar de lange-termijn uitkomsten van IBD en vooral naar de rol van medicatie. De volgende uitkomstmaten werden hierbij bestudeerd: ziekenhuisopname [hospitalisatie], chirurgie, cumulatief corticosteroïd gebruik en het optreden van complicaties van de ZvC, zoals intestinale fistels en darmstricturen.

In de Hoofdstukken 5 en 7 beschreven we dat immunomodulatoren steeds vaker en eerder worden ingezet in de behandeling van de ZvC: het cumulatieve 5 -jaar gebruik steeg van 30,6\% in de groep 1998-1998 naar 70,8\% in de groep 2006-2011. Een duidelijke stijging werd eveneens gezien bij anti-TNF $\alpha$ preparaten: van 3.1\% naar $41.2 \%$. De blootstelling aan corticosteroïden was daarentegen onveranderd [54.0\%]. Parallel aan deze veranderingen in behandelstrategie zagen we dalingen van de risico's op een ziekenhuisopname [65,9\% naar 44,2\%] en darmchirurgie [42,9\% naar 17,4\%]. Deze dalingen konden gedeeltelijk worden verklaard door een lager aantal hospitalisaties en operaties kort na diagnose van de ziekte. Dit kan duiden op veranderingen in het diagnostische proces of in de initiële behandeling van de ziekte. Voorts zagen we een afname van het cumulatieve corticosteroïd gebruik over de tijd: van een mediaan van 198 dagen in de groep 1991-1998 naar een mediaan van 0 dagen in de groep 2006-2011. Het 5-jaars risico op het ontwikkelen van darmstricturen of intestinale fistels bleef daarentegen ongewijzigd $[21,6 \%]$. Door gebruik te maken van geavanceerde statistische technieken hebben we de relatie tussen het gebruik van immunomodulatoren en anti-TNFa medicatie uitgezet tegen de bovengenoemde uitkomstmaten. Hierbij vonden we slechts een significant verschil tussen het gebruik van deze medicamenten en het nodig hebben van een kuur corticosteroïden. Dat betekent ook dat de geobserveerde verbeteringen in de risico's op ziekenhuisopname en 
darmchirurgie voornamelijk moeten worden toegeschreven aan andere factoren dan medicatiegebruik, zoals nieuwe inzichten in de rol van klinische zorg, in de rol van chirurgie en in behandelstrategieën.

In Hoofdstuk 8 bestudeerden we een invaliderende complicatie van de ZvC: perianale fisteling. We vonden dat het 5-jaars risico op het ontwikkelen van perianale fistels $[11,6 \%]$ onveranderd is gedurende de studieperiode, ondanks een frequenter gebruik van immunomodulatoren en anti-TNFa preparaten, ook vroeg in het ziektebeloop. Nadat een perianale fistel is gediagnosticeerd, staat ruim de helft van de patiënten op anti-TNF $\alpha$ therapie en desondanks wordt bij 25,7\% van de patiënten een recidief gezien binnen vijf jaar na initiële diagnose. Dit getal blijkt eveneens onveranderd te zijn over de tijd. Hierbij moet de opmerking worden gemaakt dat er laagdrempeliger beeldvormende diagnostiek kan worden ingezet naar perianale fistels, zoals een magnetic resonance imaging [MRI], hetgeen de incidentiecijfers in de recentere jaren kan hebben beïnvloed. Onze resultaten benadrukken de noodzaak tot het verbeteren van de medicamenteuze en chirurgische behandeling van perianale fistels en de noodzaak tot het optimaliseren van de initiële behandeling om de kans op perianale fistels te verkleinen.

In de Hoofdstukken 6 en 7 bespraken we de medicamenteuze behandeling en langetermijn uitkomsten van CU. Ook bij CU zagen we dat immunomodulatoren vaker worden gebruikt [8,1\% in de groep 1991-1997 en 21,7\% in de groep 2006-2010, vijf jaar na diagnose] en dat ook de tijd tot het eerste voorschrift is afgenomen. Het gebruik van antiTNF $\alpha$ middelen bleek eveneens toegenomen van $0 \%$ in de groep gediagnosticeerd tussen 1991 en 1997 naar 10,6\% in de groep gediagnosticeerd tussen 2006 en 2010. Net als bij de ZvC bleef de blootstelling aan behandeling met corticosteroïden gelijk [31,4\%]. Het risico op een ziekenhuisopname was bij CU-patiënten onveranderd [20,0\% na 5 jaar], terwijl het risico op een colectomie significant afnam van 7,5\% in de groep 1991-1997 naar 4,1\% in de groep 2006-2010. Deze daling bleek te berusten op een afname in chirurgie ten tijde van of vlak na de diagnosestelling ['vroege colectomie']. Een subanalyse naar het colectomie-risico ná zes maanden na diagnose toonde geen significant verschil tussen de groepen. Net als bij de ZvC doen deze conclusies vermoeden dat veranderingen in diagnosestelling en initiële behandeling een rol spelen. De rol van immunomodulatoren en anti-TNFa therapie is waarschijnlijk beperkt, gegeven de zeer kleine populatie CU-patiënten die al zo vroeg in het ziektebeloop aan deze middelen zijn blootgesteld. Net als bij de ZvC zagen we dat het cumulatieve corticosteroïden gebruik na het eerste ziektejaar afnam van een mediaan van 114 dagen in de groep 1991-1998 naar 94 dagen in de groep 2006-2011. Opnieuw werd een significante associatie gevonden tussen het gebruik van immunomodulatoren en antiTNFa therapie en het risico op het stootkuur corticosteroïden. 
In de studie in Hoofdstuk 9 bestudeerden we de effectiviteit van infliximab [IFX] en adalimumab [ADA] in de dagelijkse praktijk bij de ZvC en beschreven we de resultaten van een vergelijkende effectiviteitsanalyse. Anti-TNF $\alpha$ preparaten worden steeds vaker ingezet, vooral bij patiënten met kenmerken van een ernstig ziektebeloop, zoals colonische of ileocolonische ziektelokalisatie, perianale ziekte, betrokkenheid van de bovenste tractus digestivus en een positieve rookstatus. Zowel IFX als ADA toonden een duurzame respons in de dagelijks praktijk met een corticosteroïd-vrije respons in 67,1\% [IFX] en 70,1\% [ADA] na vijf jaar behandeling in primaire responders. Er werd geen significant verschil gevonden in effectiviteit tussen beide middelen. Ongeveer de helft van de patiënten herstartte anti-TNFa therapie na initieel falen, waarbij een-derde duurzame respons liet zien op tweedelijns therapie, ongeacht de reden van het initiële falen. De meeste patiënten gebruikten anti-TNF $\alpha$ therapie in combinatie met immunomodulatoren $[57,6 \%$ in het geval van IFX en $68,7 \%$ in het geval van ADA]. We vonden geen significant verschil in effectiviteit tussen patiënten op monotherapie in vergelijking met patiënten op combinatietherapie. Residuele confounding in deze analyses kan echter niet geheel worden uitgesloten, vanwege de sterkte associatie tussen combinatietherapie en ernst van de ziekte. Onze resultaten in dit hoofdstuk ondersteunen de aanname dat de keuze tussen IFX en ADA kan worden gemaakt op basis van individuele voorkeuren van de arts en de patiënt.

Tot slot werden in Hoofdstuk 10 de conclusies van alle hoofdstukken besproken en in perspectief geplaatst. Allereerst werden de uitdagingen in epidemiologisch IBD-onderzoek besproken. Het belang van het kiezen van een geschikt studiedesign en een geschikte statistische benadering werd onderstreept, waarbij in retrospectief onderzoek naar de associatie tussen medicatie en uitkomstmaten vooral rekening moet worden gehouden met 'immune-time bias'. Voorts werden de gevolgen van de stijgende prevalentie van IBD besproken en enkele suggesties gedaan om de IBD-zorg toekomstbestendig te maken. Daarna werd uitgebreid stilgestaan bij de veranderingen in de behandelstrategie en de lange-termijn uitkomsten van IBD en werden enkele nieuwe behandelstrategieën besproken. Toekomstig IBD-onderzoek moet worden toegespitst op grote 'real-world' studies om antwoorden te krijgen op prangende vragen aangaande de beste behandelstrategie voor de individuele patiënt. 



\section{VALORIZATION}

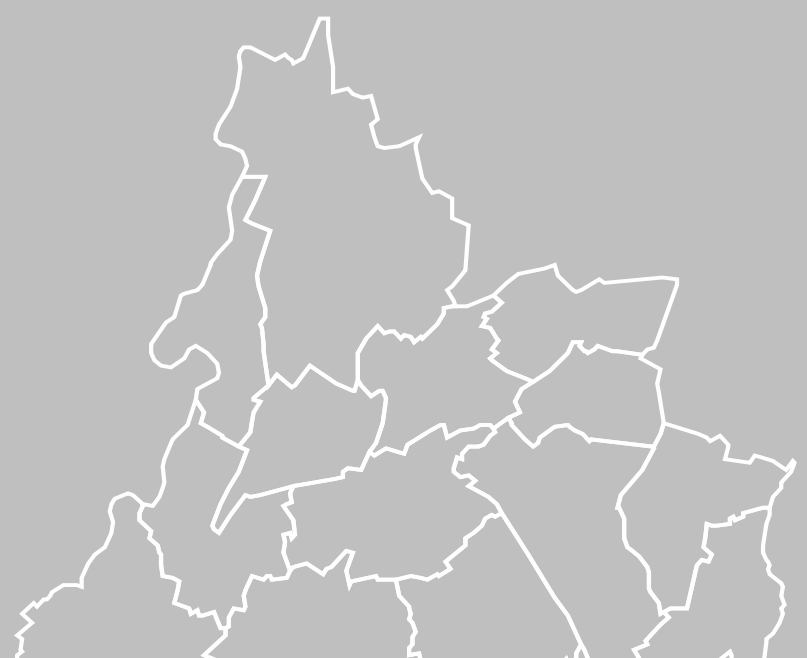




\section{Valorization}

Valorization is officially defined as "the process of value creation out of knowledge by making this knowledge suitable and available for economic and societal utilization and to translate this into high-potential products, services, processes and industrial activity".

In this chapter, the implications of our findings on the relevant socioeconomic domains of 'health care and society' and 'patients and professionals' are discussed.

\section{Implications for healthcare and society}

Chronic diseases strain the healthcare system because these can only be managed, not cured. Patients are often diagnosed at young age, resulting in a longstanding use of healthcare resources and high costs. Inflammatory bowel disease [IBD] is most often diagnosed in young adulthood [20-40 years of age] and most patients require follow-up in the outpatients' clinic. During disease course, many require expensive medical treatment and at some point inhospital medical or surgery therapy ${ }^{1-6}$, resulting in additional healthcare costs. ${ }^{7}$ Based on clinical data from IBD patients diagnosed between 1970 and 1993, Silverstein et al. estimated the life-time direct costs of Crohn's disease [CD] to be $\$ 39,906$. Major cost driver at that time was intestinal surgery, accounting for $44 \%$ of the total costs. ${ }^{7}$ More recently, Van der Valk et al. estimated the annual costs of CD in the Netherlands at € 7,835 per year. ${ }^{8}$ Apart from these direct costs, indirect costs are also noteworthy in IBD. The chronicity of the disease and its unpredictable, fluctuating course can have major impact on work, leisure time and participation in society. For instance, absenteeism and reduced work productivity are often present in IBD patients. Valid estimates of the total indirect costs of IBD are difficult to obtain, but European studies presented estimates between \$5,128 - \$14,136 per year. ${ }^{9}$ Several findings discussed in the present thesis indicated that the costs of IBD are higher than previously expected and will further increase in the near future.

First, in Chapter 3, we showed that the incidence of IBD is also increasing in the Netherlands, with annual increases of 6\% [CD] and 4\% [UC] between 1991 and 2010. Data from the most-recent years of the study period even showed higher rates. The IBD incidence in South Limburg is among the highest reported in literature ${ }^{10}$, illustrating a high disease burden in the Netherlands. Based on the South Limburg data, an estimation of the nationwide IBD prevalence was made, illustrating that 80,627 patients suffer from IBD [2010], which is much higher than previously assumed $[55,000]$. The ongoing rise in IBD incidence warrants further research on its etiology, in order to come to preventive measures. As discussed in Chapter 10, environmental factors are interesting targets, since they are modifiable and previous research have found associations between the onset of IBD and diet, smoking, urbanization and air pollution. ${ }^{11}$ However, the interaction between host and environment is 
complex and relations are preferably studied using system biology modeling, incorporating factors as from early life [exposome approach].

Second, in Chapters 5 and 6, we showed that the medical management of IBD has changed towards an earlier and more frequent use of immunomodulators and anti-TNF $\alpha$ agents. In particular, anti-TNF $\alpha$ therapy is expensive [estimated at $€ 10,000-€ 15,000$ per patient per year]. A Dutch multicenter study illustrated that current IBD healthcare costs are mainly driven by medical therapy [71\% for CD, 59\% for UC], rather than by hospitalizations and surgery [20\% for CD, 25\% for UC]. ${ }^{12}$ Because recent guidelines advocate an earlier and more frequent use of biologicals ${ }^{13}$ and new treatment strategies also propose a more frequent use of biologicals [Chapter 10], rising medication costs can be expected. Part of the expected rise will be tempered by the availability of so-called 'biosimilars', generic formulations of biological agents. Nevertheless, rising medication costs will inevitably lead to rising total healthcare costs, unless medical therapy results in an improved disease outcome. In that case, costs of frequent hospital visits, hospital admissions and surgery may attenuate. In this thesis, however, we found no significant association between the improved disease outcome and the use of immunomodulator anti-TNF $\alpha$ therapy. In literature, anti-TNF $\alpha$ therapy was already found to be cost-effective though, both as monotherapy ${ }^{14,15}$ and combination therapy ${ }^{16}$, although efficacy data mainly derive from registration trials, rather than from real-world practice. Moreover, data on cost-effectiveness on the long-term is lacking. Future studies should therefore focus on the long-term cost-effectiveness of biological therapy and future trials on new treatment strategies should include cost-utility analyses.

Third, current and future treatment strategies are aimed at 'tight control', which encompasses regular blood and stool tests, scheduled visits at the outpatient clinic and a lowthreshold use of endoscopic or radiologic modalities to assess mucosal inflammation. Such strategies can be expensive, unless they prove their selves to be cost effective in terms of better disease control and, consequent, avoidance of healthcare costs [outpatient visits, hospital admission, surgery].

Altogether, the findings reported in this thesis indicate that IBD is putting more pressure on our healthcare system than previously thought and a further increase in direct health care costs can be expected. It is of interest whether the interventions studied in this thesis result in less absenteeism and can thereby lower the indirect costs. These conclusions demand for action in order to maintain affordable, high-quality IBD care. Next to the aforementioned suggestions for further research, it is important to think of restructuring IBD care to maintain safe, high-quality care, while reducing healthcare costs. Over the past years, there has been an increasing interest in the use of eHealth tools in chronic diseases, such as congestive heart failure ${ }^{17}$ and COPD ${ }^{18}$. For IBD, the telemedicine tool 'mijnIBDcoach' is available, which follows IBD patients via monthly monitoring modules on tablet or smartphone. 
Its use was studied in a large randomized controlled trial, which showed that patients using mijn|BDcoach required less outpatient visits and hospital admissions, while patient satisfaction, risk of flares and the number of courses of corticosteroids were not different from the control group. ${ }^{19}$ Thus, telemedicine is a promising and safe management tool with the potential of being cost-effective.

\section{Implications for professionals and patients}

Both patients and professionals benefit from having up-to-date information on the IBD incidence and its course.

First, the increasing high pressure of IBD on healthcare and its observed high prevalence in the Netherlands warrants the need for more funding for scientific research. Second, by illustrating that IBD is an increasingly common disease, the present thesis also supports initiatives for improving knowledge about IBD in the general population. Better knowledge may limit patient delay in the diagnostic process and may help reducing the stigma of a disease characterized by frequent bowel movements and urgency. Third, information on the actual disease course helps physicians to educate patients better on their chronic disease, thereby improving patient empowerment. Patient empowerment and engagement in disease monitoring may subsequently lead to better treatment response, patient satisfaction and a more efficient utilization of healthcare resources. ${ }^{20}$ Information on the [long-term] disease outcome also contributes to shared decision making, for example in a discussion on the need for another medical therapy or surgery. Next to education, insight in the real-world effectiveness of current treatment strategies contributes to the development of better disease management. Real-world treatment effectiveness data can help in the process of clinical decision making in situations in which data from randomized controlled trials are lacking, which is the case in the management of specific populations [e.g. elderly] and in therapy selection [e.g. the choice of one anti-TNF $\alpha$ agent over another]. Reflecting on the latter, we observed a similar real-world effectiveness of infliximab and adalimumab in CD [Chapter 9], which emphasizes that the choice between these two agents can be made based on individual preferences of the physician or patient. In the near future, comparative effectiveness analyses in large real-world cohorts may also help in the upcoming challenge which biological therapy is best for a specific patient [personalized treatment]. Hereby, the time spent on suboptimal therapy may be minimized, which will hopefully lead to a better disease outcome and quality of life on both the short and long term. 


\section{References}

1. Solberg IC, Lygren I, Jahnsen J, et al. Clinical course during the first 10 years of ulcerative colitis: results from a population-based inception cohort [IBSEN Study]. Scand J Gastroenterol. 2009;44(4):431-40.

2. Solberg IC, Vatn MH, Hoie O, Stray N, et al. Clinical course in Crohn's disease: results of a Norwegian population-based ten-year follow-up study. Clin Gastroenterol Hepatol. 2007;5(12):1430-8.

3. Bernstein CN, Loftus EV, Jr., Ng SC, et al. Hospitalisations and surgery in Crohn's disease. Gut. 2012;61(4):622-9.

4. Jess $T$, Riis $L$, Vind I, et al. Changes in clinical characteristics, course, and prognosis of inflammatory bowel disease during the last 5 decades: a populationbased study from Copenhagen, Denmark. Inflamm Bowel Dis. 2007;13(4):481-9.

5. Rungoe $\mathrm{C}$, Langholz E, Andersson $\mathrm{M}$ et al. Changes in medical treatment and surgery rates in inflammatory bowel disease: a nationwide cohort study 1979-2011. Gut. 2014;63(10):160716.

6. Ramadas AV, Gunesh S, Thomas GA, et al. Natural history of Crohn's disease in a population-based cohort from Cardiff (1986-2003): a study of changes in medical treatment and surgical resection rates. Gut. 2010;59(9):1200-6.

7. Silverstein MD, Loftus EV, Sandborn WJ, et al. Clinical course and costs of care for Crohn's disease: Markov model analysis of a population-based cohort. Gastroenterology. 1999;117(1):49-57.

8. van der Valk ME, Mangen MJ, Severs M, et al. Evolution of Costs of Inflammatory Bowel Disease over Two Years of Follow-Up. PLoS One. 2016;11(4):e0142481.
9. Kawalec P. Indirect costs of inflammatory bowel diseases: Crohn's disease and ulcerative colitis. A systematic review. Arch Med Sci. 2016 Apr 1; 12(2): 295-30

10. Molodecky NA, Soon IS, Rabi DM, et al. Increasing incidence and prevalence of the inflammatory bowel diseases with time, based on systematic review. Gastroenterology. 2012; 142(1): 46-54 e42; quiz e30.

11. Molodecky NA, Kaplan GG. Environmental risk factors for inflammatory bowel disease. Gastroenterol Hepato/(N Y) 2010;6(5):339-46.

12. van der Valk ME, Mangen MJ, Leenders $M$, et al. Healthcare costs of inflammatory bowel disease have shifted from hospitalisation and surgery towards anti-TNFalpha therapy: results from the COIN study. Gut. 2014;63(1):72-9.

13. Gomollon F, Dignass A, Annese V, et al. 3rd European Evidence-based Consensus on the Diagnosis and Management of Crohn's Disease 2016: Part 1: Diagnosis and Medical Management. J Crohns Colitis. 2017;11(1):3-25.

14. Smart C, Selinger CP. The costeffectiveness of infliximab in Crohn's disease. Expert Rev Pharmacoecon Outcomes Res. 2014;14(5):589-98.

15. Ung V, Thanh NX, Wong K, et al. Real-life treatment paradigms show infliximab is cost-effective for management of ulcerative colitis. Clin Gastroenterol Hepatol. 2014;12(11):1871-8 e8.

16. Saito S, Shimizu U, Nan Z, et al. Economic impact of combination therapy with infliximab plus azathioprine for drug-refractory Crohn's disease: a costeffectiveness analysis. J Crohns Colitis. 2013;7(2):167-74 
17. de la Torre Diez I, Garcia-Zapirain B, Mendez-Zorrilla A, et al Monitoring and Follow-up of Chronic Heart Failure: a Literature Review of eHealth Applications and Systems. J Med Syst. 2016;40(7):179.

18. Jonkman NH, Westland $\mathrm{H}$, Trappenburg JC, et al. Do self-management interventions in COPD patients work and which patients benefit most? An individual patient data meta-analysis. Int $J$ Chron Obstruct Pulmon Dis. 2016;11:2063-74.

19. de Jong MJ, van der Meulen-de Jong AE, Romberg-Camps MJ, et al. Telemedicine for management of inflammatory bowel disease [my|BDcoach]: a pragmatic, multicentre, randomised controlled trial. Lancet. 2017;390(10098):959-68.

20. Robinson A, Thompson DG, Wilkin D, et al. Northwest Gastrointestinal Research G. Guided self-management and patientdirected follow-up of ulcerative colitis: a randomised trial. Lancet. 2001;358(9286):976-81. 



\section{LIST OF PUBLICATIONS}

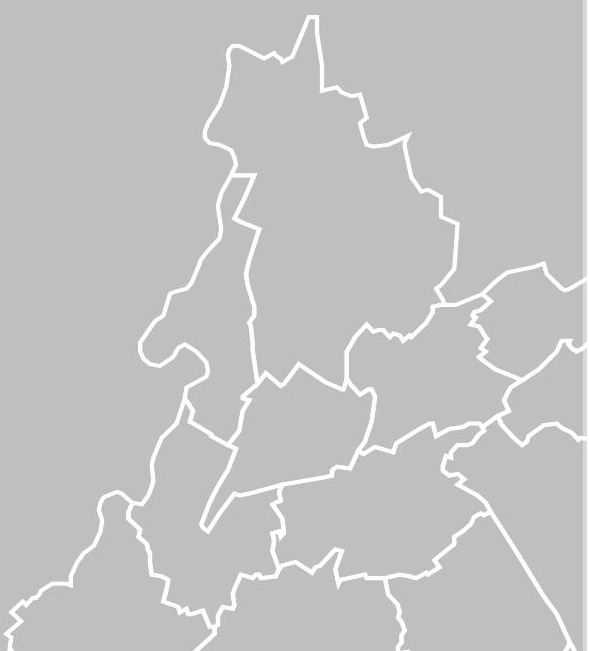




\section{Published articles}

\section{Within the thesis}

van den Heuvel TR, Jonkers DM, Jeuring SF et al., Cohort Profile: The Inflammatory Bowel Disease South Limburg Cohort [IBDSL], Int J Epidemiol. 2015 Jun 4. pii: dyv088

van den Heuvel TR*, Jeuring SF*, Zeegers MP et al., A 20 year temporal change analysis in incidence, presenting phenotype and mortality in the Dutch IBDSL cohort - Can diagnostic factors explain the increase in IBD incidence?, J Crohns Colitis. 2017 Oct 1;11[10]:11691179.

Jeuring SF, Van den Heuvel TR, Zeegers MP et al., Epidemiology and long-term outcome of inflammatory bowel disease diagnosed at elderly age - an increasing distinct entity?, Inflamm Bowel Dis. 2016 Jun;22[6]:1425-34

Jeuring SF, Van den Heuvel TR, Liu LY et al., Improvements in the long-term outcome of Crohn's disease over the past two decades and the relation to changes in medical management: results from the population-based IBDSL cohort, Am J Gastroenterol. 2017 Feb;112[2]:325-336

Jeuring SF, Bours PH, Zeegers MP et al., Disease outcome of ulcerative colitis in an era of changing treatment strategies - Results from the Dutch population-based IBDSL cohort, J Crohns Colitis. 2015 Oct;9[10]:837-45

Jeuring SF, Biemans VB, van den Heuvel TR et al., Corticosteroid sparing in inflammatory bowel disease in more often achieved in the immunomodulator and biological era - Results from the population-based IBDSL cohort, Am J Gastroenterol. 2018 Mar;113[3]:384-395

Göttgens KW*, Jeuring SF*, Sturkenboom R et al., Time trends in the epidemiology and outcome of perianal and rectovaginal fistulizing Crohn's disease in a population-based cohort, Eur J Gastroenterol Hepatol. 2017 May;29[5]:595-601

\section{Outside of the thesis}

Jeuring SF, van den Heuvel TR, Zeegers MP et al., Minder ziekenhuisopnames, operaties en gebruik van corticosterö̈den bij de ziekte van Crohn, Ned Tijdschr Geneeskd. 2017; 161[0]:D1641

Sturm A, Maaser C, ... , Jeuring SF et al., European Crohn's and Colitis Organisation Topical Review on IBD in the Elderly, J Crohns Colitis. 2017 Mar 1;11[3]:263-273 
van den Heuvel TR, Wintjens DS, Jeuring SF, Inflammatory bowel disease, cancer and medication: Cancer risk in the Dutch population-based IBDSL cohort, Int J Cancer. 2016. May 11

Van Hoof M*, Jeuring SF*, Stokroos RJ et al., A new perspective on measuring quality of life using the capability approach, Ned Tijdschr Geneeskd. 2015;159[0]:A9234

Van Hoof M*, Jeuring SF*, Jonkers DM et al., De Nederlandse vertaling en indruksvalidatie van de ICECAP-A: meten van kwaliteit van leven volgens de capability-benadering, Tijdschr v Gezondheidsw. 2016 94[8]:-306-313

\section{Submitted articles}

Jeuring SF, Wintjens DS, Joore MA et al., Real-world long-term effectiveness and comparative effectiveness of infliximab and adalimumab in a population-based cohort of Crohn's disease patients

\section{Awards}

2018 Young ICC Research Award Improvements in the long-term outcome of Crohn's disease over the past two decades and the relation to changes in medical management

2016 Young ECCO Abstract Award

Evolution of corticosteroid use in Crohn's disease patients between 1991 and 2014 results from the Dutch population-based IBDSL cohort

2015 Best of Digestive Disease Week 2015

Disease outcome of Crohn's disease - has the disease outcome improved over the last two decades?

2014 Young ECCO Abstract Award Is elderly-onset ulcerative colitis a different entity? - Natural disease course and treatment response compared to adult-onset disease in the population-based IBDSL cohort

\section{Nomination 'Pélerin Wetenschapsprijs' 2013}

The availability of anti-tumor necrosis factor agents is associated with changes in the natural disease course of ulcerative colitis - a population-based study in the IBDSL cohort 



\section{Dankwoord}

Als mij een ding duidelijk is geworden gedurende mijn promotietijd, dan is het wel dat wetenschappelijk onderzoek een teamsport pur sang is. Bij het opstellen van een hypothese, het bedenken van een onderzoeksmethode, het verzamelen van data, het analyseren van gegevens en bij het presenteren van conclusies in presentatie of schrift; in alle gevallen kom je er alleen met een sterk team aan mensen om je heen. Dit zal ongetwijfeld de reden zijn dat juist dit hoofdstuk het best-belezen hoofdstuk van een thesis is.

In de komende bladzijden wil ik dan ook graag stilstaan bij allen die in de afgelopen jaren hebben bijgedragen aan de totstandkoming van dit proefschrift. Sommigen direct door input te leveren op de gepresenteerde stukken, anderen indirect door het verzamelen en verwerken van gegevens te faciliteren of de persoon in kwestie te ondersteunen en motiveren. Enkele mensen wil ik graag in het bijzonder bedanken voor hun bijdrage aan dit boekje.

Professor Masclee, beste $A d$, ik zie mijzelf nog bij u op het kantoor zitten aan het einde van mijn keuze-coschap bij de maag-darm-leverziekten. Mijn enthousiasme over deze korte stage werd door u gekatalyseerd en ik ben u dankbaar voor de mogelijkheid die ik kreeg om enkele jaren later terug te keren om een volledig promotieonderzoek uit te voeren. In de onderzoeksperiode wist u altijd in uw feedback de conclusies van de artikelen aan te scherpen. Bedankt voor het vertrouwen dat $u$ heeft in mijn functioneren en in mij als persoon om een bedreven MDL-arts te worden.

Dr. Pierik, beste Marieke, toen ik in september 2011 begon als WESP'er bij het IBD-team had ik nooit kunnen bedenken dat wij 8 jaar later een volledig proefschrift zouden afleveren. Je enorme enthousiasme over IBD en research heeft op mij zo z'n gevolgen gehad en ik geloof dat ik er nog blij mee ben ook. Ik ken weinig mensen die zo gedreven en gepassioneerd zijn. Jouw ideeën zijn de basis van dit proefschrift en ik ben je enorm dankbaar voor de vele mooie publicaties en presentaties die hieruit voortvloeiden. In het bijzonder wil ik je bedanken voor het vertrouwen dat je altijd hebt gehad in de methodologische aanpak van mijn studies en in de uitwerking van presentaties ['het is jouw presentatie hè, Steven!']. Bedankt voor de fantastische tijd en je belangrijke rol bij het behalen van mijn research-rijbewijs!

Professor Jonkers, beste Daisy, jij en Marieke zijn een gouden duo. Waar Marieke gepassioneerd minstens tien nieuwe onderzoeksideeën per IBD-bespreking met ons deelde, wist jij er te allen tijde zorg voor te dragen dat projecten haalbaar waren en werden afgerond. Ook je bijzonder scherpe wetenschappelijke inzicht heeft de soms complexe methodologie van onze studies verhelderd en zorgde altijd voor duidelijkere conclusies. Een unieke eigenschap die ik in het bijzonder enorm heb gewaardeerd wil ik eruit lichten: je vermogen om elke [lees: elke] tekst met minimaal 50\% in te korten zonder verlies van de essentie of leesbaarheid. Zonder jouw bijdrage waren de drukkosten van dit boekje aanzienlijk hoger geweest. Je hebt 
een aardige kluif gehad aan het 'wollige' schrijven van mij, dus alleen daarvoor al een heel oprechte en grote dankjewel!

De leden van de beoordelingscommissie, te weten prof. dr. Boonen, prof. dr Spruit, prof. dr. Bouvy, dr. Oldenburg en dr. Wolters, wil ik graag bedanken voor het kritisch beoordelen van dit proefschrift.

Beste Tim, mijn IBD-partner in crime. Dat dit boekje niet tot stand was gekomen zonder de fijne samenwerking met anderen is vooral op jou van toepassing. En dat bedoel ik in de breedste zin van het woord. Vele uren spendeerden we aan [kritische] discussies over elkaars studieopzetten, statistische benaderingen, conclusies en schrijfwijzen. Deze discussies hebben enorm bijgedragen aan de kwaliteit van al onze studies, maar zeker ook aan mijn inzicht in de wetenschap. Maar ook als collega en vriend heb ik je enorm gewaardeerd, dankjewel daarvoor. En wat betreft stijl en smaak, tja, laten we zeggen dat er een wederzijds respect bestaat. Het voelt fijn dat je als paranimf aan mijn zijde staat.

Beste Bas, onze levens lopen al heel lange tijd parallel en dat heb ik nog geen moment als vervelend ervaren! Het 'samen promoveren' leek aanvankelijk wat problematisch te worden, maar werd door Marieke en professor Masclee uiteindelijk naar ieders tevredenheid opgelost, zodat er uiteindelijk toch twee 'doctors' MDL binnen de HEEP10 zijn. Over zowel strubbelingen op het werk als daarbuiten konden we goed praten onder het genot van een goed glas wijn op het terras. Dankjewel daarvoor. Het moge duidelijk zijn dat ik je heel graag als paranimf aan mijn zijde heb staan.

Beste Mariëlle, Liekele en Wim. Afgelopen jaren heb ik de luxe mogen ervaren van een zeer prettige samenwerking tussen de drie ziekenhuizen. Het spreekt voor zich dat het verzamelen van de gegevens meer inspanning en [nog] meer tijd had gekost als de communicatie niet zo soepel was gelopen. Ook jullie motiverende woorden tijdens het doorspitten van de dossiers en als reactie op de manuscripten heb ik enorm gewaardeerd. Mariëlle, een extra dankjewel voor al je inspanningen die je hebt verricht eerder in de 'IBDZL-trein'. Jouw promotieboekje was een inspiratie voor de mijne, blijkens ook de staat van jouw boekje aan het einde van mijn rit: het moet helaas worden bestempeld als 'verbruiksmateriaal'.

Professor Zeegers, beste Maurice, bedankt voor je hulp bij het beoordelen van de methodologie van de studies in deze thesis. Het voelt fijn dat bij een groot cohort als IBDZL een 'eigen' epidemioloog voorhanden is. Daarbij waren je zorgvuldige commentaren altijd bijzonder zinvol, juist ook vanwege je methodologische invalshoek en het feit dat je als buitenstaander naar de materie kon kijken. Bedankt daarvoor.

Beste Kevin, professor Stassen en Dr. Breukink. IBD is een ziekte die zich niet beperkt tot de MDL-arts en daarom is het fijn dat in dit proefschrift ook jullie input als chirurgen is vertegenwoordigd. Het heeft geleid tot een heel prettige samenwerking voor nu en ook voor 
de toekomst. Kevin, het heeft even geduurd voordat onze 'fistel-paper' was geland, maar de aanhouder wint. Bedankt voor je inzet en geduld en veel succes in je carrière!

Professor Joore, beste Manuela, bedankt voor je hulp bij de effectiveness-paper. Je hebt me geleerd efficacy en effectiveness van elkaar te onderscheiden; waardevolle informatie voor mij als onderzoeker, maar zeer zeker ook voor mij als clinicus. Ook heb je me laten proeven van het kwalitatieve onderzoek met de ICECAP-A, hetgeen ik als een bijzonder leuk 'uitstapje' heb ervaren! Beste Marc, bedankt voor de fijne samenwerking en je engelengeduld. Het heeft even geduurd voordat de ICECAP-A paper door een tijdschrift was geaccepteerd, maar hier gold zeker dat de weg ernaar toe minstens zo belangrijk en leerzaam is geweest als het uiteindelijke doel. Succes met je verdere carrière!

Beste Mia, Henny, en Yvan, jullie waren tijdens de promotiejaren een aangenaam uitstapje van de universiteit naar het ziekenhuis. Yvan, je levenswijsheden zullen ervoor zorgen dat ik de komende tientallen jaren het leven positief en vrolijk tegemoet ga. Mia en Henny, dankjulliewel voor de begeleiding bij 'mijn eerste stapjes in de kliniek' tijdens de telefonische spreekuren. Leerzaam en bovendien goed voor de lachspieren. Door alle telefoongesprekken met patiënten is mij duidelijk geworden dat jullie als persoon enorm worden gewaardeerd en dat kan ik beamen. Jullie hebben mij laten zien dat goede IBD-zorg niet mogelijk is zonder goede, menselijke, gespecialiseerde verpleegkundigen.

Beste studenten, in het bijzonder Vince en Limmie, wat heb ik met jullie geboft! Met minimale aansturing waren jullie in staat gegevens op een juiste manier te verzamelen, te verwerken en te analyseren. Jullie kostten geen energie, maar gaven mij juist energie met jullie enthousiasme en kritische vragen! Juist daarom vind ik het fantastisch dat jullie zijn vertegenwoordigd op twee artikelen in deze thesis. Vince, hoe chic is het dat jij een van de volgende in de rij IBDpromovendi bent!

Zeer bepalend voor mijn herinnering aan de PhD-tijd waren jullie, lieve MDL-collega's. Natuurlijk mijn roomies Tim, Zlatan en later Roy. Zlatan, ik hoop dat je ook ooit nog eens tot voetbalkampioen wordt gekroond door een onafhankelijke jury. Roy, met veel plezier kijk ik terug op de lange farmaco-epidemiologische discussies, ook al was je vaak moeilijk te overtuigen... Het was ook fijn om matties te zijn geweest in het Venlose. Ook een bijzonder dankjewel aan mijn IBD-collega's Marin, Danyta en Dion. Marin, wat ben je toch heerlijk anders dan ik ben. Danyta, je hebt een ongekend doorzettingsvermogen en bewonderenswaardige vriendelijkheid over je. Dion, ik kijk uit naar alle studies die je gaat uitvoeren die voortborduren op de stukken in deze thesis. Dankjewel Pauline, Zsa Zsa, Lisa, Martine, Montserrat, Fedde, Fabiënne, Ellen, Annick, Chantal, Bauke, Wiesje, Hao Ran, Ankie, Kirsten, Selwyn, Tim K, Bram en Evelien. Of het nu de lunches, koffiepauzes of activiteiten buiten het werk waren, het was altijd gezellig. Perfecte momenten om even stoom af te blazen of het computerscherm te ontvluchten. Onvergetelijk waren ook de filmpjes; bedankt Mark en Roe/voor het compileren ervan. Zeker ook de wintersport ["Unheimlich steif"] staat in mijn geheugen gegrift. 
De mooiste momenten dienen zich altijd aan in het buitenland en ik ben dankbaar dat ik zoveel keren tripjes heb mogen maken naar congressen. Elke stad met eigen verhalen: Kopenhagen [waar ik heb geleerd dat Tim ver gaat voor vrienden, ook na een avondje stappen], Barcelona [dineren in het 'Museu Nacional d'Art de Catalunya'], Orlando [Senor Frog, need I say more?], Washington [op muzikaal gebied is 'fout' een dubieus begrip], San Diego [stad van IPA en 'congres-spijbelen' samen met de 'bazen']. Fantastische ervaringen, bedankt allemaal!

Na alle mooie PhD-jaren dreigde het beruchte 'zwarte gat'. Niets is minder waar, dankzij de fantastische assistentenclub van het Viecuri! Dankjewel Loes, Iris, Simon, Robbert-Jan, Brecht, Roy C, Heleen, Maroeska, Marian, Heloise, Fleur, Jenny, Yvette, Marlou, Kevin, Marissa, Pauline, Davy, Karen, Anke, Karin, Doaa, Lisanne en natuurlijk Bas, Roy en Kirsten. Dank ook aan alle internisten en maag-darm-leverartsen van het Viecuri voor hun interesse in mijn onderzoek en begeleiding in de kliniek. Dr. Hermans, beste Marc, bedankt voor je interesse in de voortgang van mijn promotietraject en het begrip voor de soms moeilijke combinatie van kliniek, promotie en gezin voor een "VOP'er".

Ook de collega's in het MUMC wil ik bedanken voor hun interesse tijdens de laatste fase van het proefschrift: Fabiënne, Ankie, Bas, Chantal, Corinne, Golnar, Jischmaël, Kirsten, Maartje, Mark van Boekel, Mark van Avesaat, Marlous, Pauline, Susan, Wesley, Zlatan en Philippe, jullie zijn toppers!

Nienke en Elly, bedankt voor jullie ondersteuning, vooral bij de laatste [officiële] loodjes van het proefschrift!

Aangezien werk en privé vaak door elkaar heen liepen, in de promotietijd zelf, maar vooral in de periode nadien, ben ik mijn vrienden en familie minstens zoveel dank verschuldigd.

Lieve vrienden, bedankt voor alle gezellige momenten buiten het werk om die mij de energie gaven om weer goed gemutst op de werkvloer te verschijnen. In het bijzonder een dankjewel aan de HEEP10-vriendengroep. Lieve Annique, Bas, Valéry, Ruud, Lonneke, Bram, Rob en Elianne, wat fijn om te merken dat er vriendschap in deze vorm bestaat. Proost op nog vele gezellig etentjes en weekendjes weg!

Lieve papa, mama en Martijn, vooral dankzij jullie ben ik gekomen tot waar ik nu ben. Ook al heb ik tijdens mijn jaren promotie en opleiding veel geleerd, jullie hebben mij bijgebracht wat écht belangrijk is in het leven: het volgen van je hart en doen wat goed voelt; dan komt het altijd goed en kom je overal. Al was het wat onverwacht dat ik een 'wetenschappelijk wandelpad' ging belopen, jullie steunden mij volledig en waren oprecht geïnteresseerd, al was het soms wat lastig te begrijpen waar ik nu precies mee bezig was. Dankjulliewel voor alles!

De laatste alinea van dit boekje is voor de drie dames die mijn leven rijk en compleet maken en minstens zo blij zijn als ik dat het proefschrift is afgerond. Lieve Lian, al jaren mijn steun en toeverlaat. Onvoorwaardelijk respecteer je mijn [soms ambitieuze] keuzes, al hebben die nogal 
eens impact op ons gezinsleven. Ik heb enorme bewondering voor je flexibiliteit en planningskwaliteiten. Jij zorgde ervoor dat alles bleef draaien, waardoor ik de tijd kreeg om kliniek, promotie, gezin en mijzelf draaiende te houden. Daar ben ik je ongelooflijk dankbaar voor!

Lieve Evien Marin. Als jullie een beetje groter zijn dan zullen jullie snappen waarom papa zoveel uren heeft gewerkt aan een boek zonder mooie plaatjes of spannende avonturen over konijntjes of biggetjes. Papa houdt heel veel van jullie! 



\section{CURRICULUM VITAE}

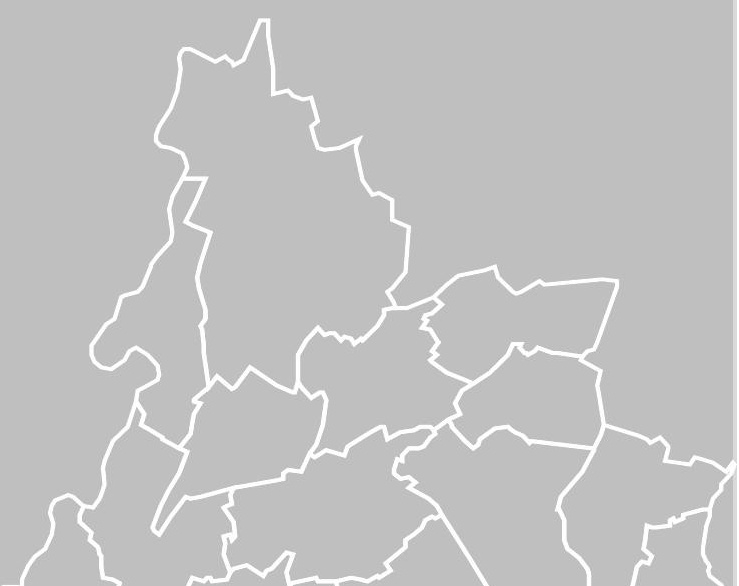




\section{Curriculum vitae}

Steven Franciscus Gerardus Jeuring was born on July, $29^{\text {th }}, 1987$ in Enschede, the Netherlands. After graduation from the Twickel College in Hengelo in 2005, he obtained a propedeuse in Medical Biology at the Radboud University in Nijmegen in 2006. Afterwards, he started medical school at the Maastricht University, Faculty of Health, Medicine, and Life Sciences. The field of Gastroenterology-Hepatology first drew his attention in 2010 during an internship in the Maastricht University Medical Center. In 2011, he started a scientific traineeship on inflammatory bowel disease, under supervision of dr. M.J. Pierik, during which the work presented in this thesis was initiated.

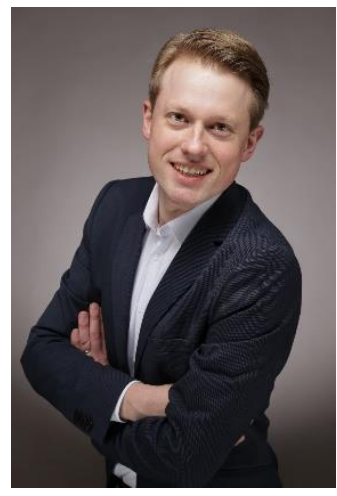

After obtaining his medical degree in June 2012, he returned to Maastricht to start as PhD fellow at the department of Internal Medicine, division of Gastroenterology-Hepatology within NUTRIM, School of Nutrition and Translational Research in Metabolism, under supervision of prof. dr. A.A.M. Masclee, dr. M.J. Pierik and prof. dr. D.M.A.E. Jonkers. During the PhD tract, part of the work outlined in this thesis was presented in oral presentations at international conventions and was awarded twice with the Young ECCO Abstract Award. Between 2014 and 2016, Steven was also chairman of the Young Initiative on Crohn's and Colitis [Y-ICC], a national network for young scientists and physicians interested in inflammatory bowel disease.

At October, $1^{\text {st }}$ 2016, he started his residency in Gastroenterology-Hepatology under supervision of prof. dr. A.A.M. Masclee. During the first two years, he worked at the department of Internal Medicine at Viecuri Hospital in Venlo, under supervision of dr. M.M.H. Hermans. As of August 2018, he continues his residency in the Maastricht University Medical Center. 

